\title{
Experiments with a Spectral Tropical Cyclone Model
}

\author{
by \\ Mark DeMaria \\ Department of Atmospheric Science \\ Colorado State University \\ Fort Collins, Colorado
}

\section{Department of \\ Atmospheric Science}

Paper No. 371 
EXPERIMENTS WITH A SPECTRAL TROPICAL CYCLONE MODEL

by

Mark DeMaria

Research supported by the National Science Foundation under

Grant ATM-8207563 and by the

Office of Naval Research

under Grant N00014-83-K-0068

(Principal Investigator: Wayne H. Schubert)

Department of Atmospheric Science

Colorado State University

Fort Collins, Colorado

July, 1983

Atmospheric Science Paper No. 371 


\section{ABSTRACT OF THESIS \\ EXPERIMENTS WITH A SPECTRAL TROPICAL CYCLONE MODEL}

The three-layer balanced axisymmetric tropical cyclone model presented by Ooyama (1969a) is generalized to three dimensions and the resultant primitive equations are solved using the spectral (Galerkin) method with Fourier basis functions on a doubly-periodic mid-latitude $\beta$ plane. The nonlinear terms are evaluated using the transform method (Orszag, 1970; Eliasen et al., 1970) where the necessary transforms are performed using FFT algorithms. The spectral equations are transformed so that the dependent variables represent the normal modes of the linearized equations. For the three-layer model the normal modes correspond to internal or external gravity or rotational modes or to inertial oscillations associated with the constant depth boundary layer. When the governing equations are written in terms of the normal modes, the application of the nonlinear normal mode initialization scheme proposed by Machenhauer (1977) is straightforward. For the initialization scheme, the rotational modes and inertial oscillations are defined as the slow modes and the gravity modes are defined as the fast modes.

The model is run with an axisymmetric initial condition on an $f$ plane and it is shown that many of the results presented by 0oyama (1969a) can be reproduced. The energy of the gravity modes and rotational modes are calculated for this simulation and it is shown that the gravity mode energy is more than an order of magnitude smaller than 
the rotational mode energy. This provides some justification for the use of periodic boundary conditions. The model is then run on the $\beta$ plane and it is shown that the variation of the Coriolis parameter with latitude causes the tropical cyclone' to move towards the northwest at about $2 \mathrm{~ms}^{-1}$, in agreement with several other authors. It is also shown that the dispersion of the rotational modes causes the tropical cyclone to elongate towards the west and develop sharper geopotential gradients towards the east. These features are similar to features of storms found in nature. The model is also run with a basic state wind profile and it is shown that the interaction between the storm circulation and the basic state wind causes large asymmetries to develop. The effect of the basic state wind on the storm size and intensity is also studied. It is shown that the basic state wind in the upper layer can interact with the storm outflow to either increase or decrease the storm intensification rate, while the basic state in the lower layer can affect the intensification rate as well as the size of the model tropical cyclone.

The effect of initialization procedures on a tropical cyclone forecast is also studied. The results from linear and nonlinear normal mode initialization procedures and results from applying an initialization procedure based on the nonlinear balance equation are compared. It is shown that the nonlinear normal mode initialization procedure results in much smaller track and intensity forecast errors, and prevents the excitation of spurious gravity waves.

Several examples of tropical cyclone motion in the nondivergent barotropic model are presented. It is shown that this simple model can predict the track of the tropical cyclone in the primitive equation 
model except during periods of rapid intensification. It is also shown that the spectral truncation, horizontal diffusion coefficient and the tangential wind profile outside of the radius of maximum wind can each affect the track of a tropical cyclone in the barotropic model. 


\section{ACKNOWLEDGEMENTS}

The author would like to thank Professor Wayne H. Schubert for his support and guidance throughout this work, and also Professors William M. Gray, Duane E. Stevens, and Gerald Taylor for their comments. I also received valuable help from Mr. Scott R. Fulton, Dr. Pedro L. Silva Dias, and Dr. James J. Hack.

I would also like to thank Ms. Odilia Panella for her patience in typing the manuscript and Ms. Judy Sorbie for drafting the figures.

This research was supported by the National Science Foundation under Grant ATM-8207563 and the Office of Naval Research under Grant N00014-83-K-0068. Acknowledgement is also made to the National Center for Atmospheric Research, which is sponsored by the National Science Foundation. 
TABLE OF CONTENTS

$\underline{\text { Page }}$

1.0 INTRODUCTION.................................... 1

2.0 GOVERNING EqUATIONS................................ 10

2.1 Large scale governing equations.................... 10

2.2 Specification of friction terms.......................... 14

2.3 Cumulus parameterization............................, 15

2.4 Equations of motion on a mid-latitude $\beta$-plane........... 22

2.5 Summary of the governing equations................... 27

3.0 SOL.UTION USING THE FOURIER-GALERKIN METHOD $\ldots \ldots \ldots \ldots \ldots \ldots \ldots, 31$

3.1 Spectral form of the governing equations.............. 35

3.2 Evaluation of the nonlinear terms using the transform method............................... 41

3.3 Derivation of the model normal modes................... 44

3.4 Time discretization............................... 57

3.5 Nonlinear normal mode initialization................... 60

3.6 High wavenumber filtering....................... 61

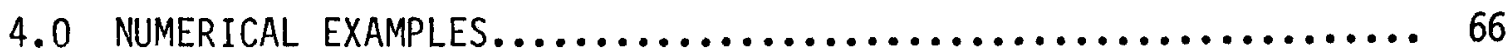

4.1 Simulation of an axisymmetric tropical cyclone on an $f-p l a n e . . . \ldots \ldots \ldots \ldots \ldots \ldots \ldots \ldots . . . \ldots 6$

4.2 The effect of a variable Coriol is parameter on a tropical cyclone simualtion..................... 94

4.3 Simulation of a tropical cyclone with a nonresting basis state............................. 105

4.4 Transformation of a wavelike disturbance into a closed vortex.............................. 118

5.0 THE INFLUENCE OF THE LARGE-SCALE FLOW ON TROPICAL CYCLONE INTENSIFICATION...................... 130

5.1 The effect of horizontal wind shear in the lower layers................................ 131

5.2 The effect of horizontal wind shear in the upper layer.................................. 143

5.3 Interaction with a trough in the upper layer............ 150 
6.1 The effect of nonlinear normal mode

initialization on a tropical cyclone track forecast....... 162

6.2 Tropical cyclone motion in the nondivergent

barotropic model............................... 174

6.3 The effect of spectral truncation, horizontal

diffusion and vortex size on nondivergent

barotropic model track forecasts................... 182

7.0 SUMMARY AND CONCLUSIONS.......................... 195

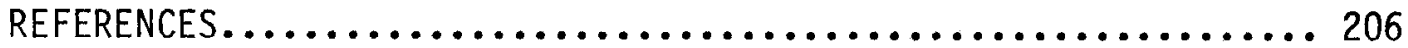

APPENDIX A. The Use of Fast Fourier Transform (FFT)

Algorithms in the Transform Method............ 212

APPENDIX B. Properties of the Normal Mode Transform......... 220 
CHAPTER 1

INTRODUCTION

The tropical cyclone, with its intense low-level wind and torrential rain, must rank among the most amazing and interesting meteorological phenomena. Observational and theoretical studies have shown that the existence of tropical cyclones is due to a complex interaction between the synoptic scale and cumulus convection which, through latent heat release, provides the energy to drive the system. This interaction between scales of motion, which are dynamically quite different, makes tropical cyclone forecasting a difficult task and presents a challenging problem for numerical modelers.

Linear theory of a convectively unstable atmosphere indicates that the disturbances with the smallest horizontal scales will grow the fastest (Bjerknes, 1938). This appears to indicate that convective instability will not lead to the growth of a disturbance with a horizontal scale as large as a tropical cyclone. Ooyama (1964) and Charney and Eliassen (1964) showed that the cumulus clouds and the larger scale tropical cyclone circulation could enhance each other. Although this early work, which was based on linear theory, could not explain the development of a tropical cyclone to the mature stage, it did introduce the concept of a cooperation rather than competition between the different scales of motion. This cooperative interaction is often referred to as Conditional Instability of the Second Kind (CISK). 
One of the first numerical simulations which produced the development of a vortex similar to an average tropical cyclone was presented by Doyama (1969a, 1969b). Ooyama's model was axisymmetric and balanced, and treated the large scale environment as three layers of incompressible fluid. The effects of cumulus clouds were included as a mass transport between two of the fluid layers. The magnitude of the mass transport was proportional to the vertical velocity at the top of the boundary layer and to a vertical stability parameter which depended on the vertical distribution of temperature and moisture. This implicit treatment of the effects of cumulus clouds is a relatively simple example of what is now referred to as cumulus parameterization.

Since the early work of Ooyama and others, many more sophisticated models have been developed. As computers became more powerful, vertical and horizontal resolution increased and more complicated cumulus parameterization schemes could be used. For example, Kurihara and Tuleya (1981) used an 11-level primitive equation model to study the development of a wave into a closed vortex and Hack and Schubert (1981a) used a parameterization scheme which considered an ensemble of cumulus clouds. A comprehensive review of the development of tropical cyclone models can be found in Anthes (1982).

Although the physics and geometry used in tropical cyclone models have become more sophisticated, the numerical methods used to solve the governing equations have remained relatively constant. The basic modeling procedure has been to write finite difference approximations to the governing equations and then to solve these approximate equations for the values of the dependent variables on some specified grid. An alternate approach to finite difference methods is to use a spectral 
method. In spectral methods the spatial dependence of the dependent variables is represented by a finite series of an appropriate set of basis functions. The governing equations are then used to give a set of ordinary differential equations for the time dependent amplitudes of the finite series which are usually solved using time differencing. Spectral methods have been used on many problems in meteorology and fluid dynamics (see Machenhauer (1979) and Orszag (1979) for examples) and have been shown to be much more accurate per degree of freedom compared to finite difference methods. Spectral methods can also reduce computational dispersion which is caused by an artificial reduction in the phase speed of waves with wavelengths close to twice the grid spacing in a finite difference model. With careful evaluation of nonlinear terms, the nonlinear instability of the type described by Phillips (1959) can also be eliminated with the use of spectral methods. Despite these computational advantages, spectral methods have never been applied to a tropical cyclone model.

In this thesis, a three-dimensional primitive equation tropical cyclone model will be developed and solved using a spectral method. The physical model is based on the early tropical cyclone model developed by Ooyama (1969a). His model is generalized by relaxing the axisymmetric and balance assumptions and including a variable Coriolis parameter. Doyama's model was chosen for study because it contained a relatively simple cumulus parameterization scheme and vertical structure, yet was capable of reproducing many aspects of tropical cyclones found in nature. Bliss (1980) used a generalized version of Doyama's model and showed that this system of equations is also capable of simulating the transformation of a wave into a closed vortex. 
In meteorology, the term spectral method usually refers to a specific form of the spectral method known as the Galerkin method. In the Galerkin method, the dependent variables are expanded in a finite series of basis functions which are orthogonal with respect to some inner product and satisfy the same boundary conditions as the dependent variables. The equations for the time dependent amplitudes are found by substituting the series expansions into the governing equations, and then taking the inner product of the equations with each of the basis functions. For global or hemispheric problems, spherical harmonics, Hough functions and trigonometric functions have been used as basis functions. In limited area models the boundary conditions are usually more complicated and it is often difficult or impossible to find basis functions which satisfy these conditions and are also appropriate to use for a series expansion. It is possible, however, to simulate an infinite domain by considering a domain which is periodic in both the $x$ and $y$ directions, but is relatively large compared to the size of the disturbance being studied. The appropriate basis functions for the Galerkin method are then Fourier components, and the series expansions become truncated double Fourier series. This method will be used to solve the governing equations for the generalized version of 0oyama's model.

The method of solution presented here can be generalized to include more complicated boundary conditions by using a spectral method known as the tau method (Gottlieb and Orszag, 1977). In the tau method, the dependent variables are expanded in truncated series, but the basis functions are not required to satisfy the boundary conditions individually. Extra degrees of freedom are added in such a way that the 
series as a whole satisfies the boundary conditions. The appropriate basis functions for this case are Chebyshev polynomials. Haidvogel et al. (1980) have used this method in an ocean model with open boundaries. A disadvantage of this method, however, is that the Chebyshev polynomials oscillate rapidly near the boundaries. This extra boundary resolution makes it necessary to use a very small time step or an implicit time differencing scheme. For this reason, only Fourier basis functions will be used here.

Another advantage to using Fourier bas is functions is that the Fourier components are proportional to the normal modes of the linearized governing equations. It will be shown that this makes it possible to evaluate the linear terms exactly by multiplying the governing equations by appropriate integrating factors. This allows the use of a time step larger than required by the CFL criterion for external gravity waves since the motion of these waves are governed by the linear terms. The fact that the basis functions are proportional to the normal modes also makes the application of nonlinear normal mode initialization relatively straightforward. In chapter three it will be shown how the nonlinear normal model initialization procedure introduced by Machenhauer (1977) can be applied in the current model.

After the Galerkin procedure is applied, the governing equations are transformed into a series of ordinary differential equations for the Fourier coefficients. When these equations are solved using time differencing it is necessary to evaluate the transform of the nonlinear terms at each time step. Historically, this restricted early spectral models to very low resolution since the method used to calculate the nonlinear terms (the interaction coefficient method) required large 
numbers of arithmetric operations and large amounts of computer storage. For the interaction coefficient method, the nonlinear terms are computed by directly multiplying series expansions together and then multiplying each term by the appropriate interaction coefficient which takes into account the inner products of the various combinations of basis functions which appear. Not only was this method inefficient, but it also made the inclusion of physical processes difficult since at any given time, only the series amplitudes were known. These difficulties were removed when a new method for computing the nonlinear terms (the transform method) was introduced by Orszag (1970) and Eliasen et al. (1970). For the transform method, the nonlinear terms are computed by first transforming the dependent variables to physical space on some specified grid. The nonlinear products are then calculated at the grid points and the inverse transform is evaluated using an appropriate numerical quadrature rule. Except for very low spectral truncations, the transform method is more efficient than the interaction coefficient method and also eliminates the need for large amounts of storage for the interaction coefficients. The transform method also makes the inclusion of physical processes straightforward since the dependent variables are calculated in physical space at each time step. It will be shown that when the transform method is used with Fourier basis functions, both the transforms and inverse transforms can be written in terms of discrete Fourier transforms so that Fast Fourier Transform (FFT) algorithms can be applied to greatly improve the model efficiency.

The development of the physical model will be discussed in Chapter 2 and the details of the solution using the Galerkin method with Fourier basis functions will be presented in Chapter 3. In Chapter 4, results 
from four simulations will be presented. The model is run with an axisymmetric initial vortex on an f-plane and on the $\beta-p l a n e$. The model is also run on the $\beta-p l a n e$ with a nonzero zonal wind field and with a wave-like initial condition. These results show that the current model can reproduce many of the results presented by Ooyama (1969a) and Bliss (1980), and also can simulate many aspects of tropical cyclones observed in nature. Since the model is formulated in terms of the normal modes of the linearized governing equations, the amplitudes of the gravity and rotational modes are known explicitly. In Chapter 4, the energy of various modes of the solution is calculated for a tropical cyclone simulation.

Tropical cyclones which occur in nature exhibit a wide variation in size and strength (e.g. Merri11, 1982). The development of weaker tropical disturbances is also highly variable, and extremely difficult to forecast. For example, during a typical Atlantic hurricane season, only about 9 out of 27 tropical depressions develop into tropical storms (winds $>17 \mathrm{~ms}^{-1}$ ) and only about 5 develop into hurricanes (winds > 33 $\mathrm{ms}^{-1}$ ) (Herbert, 1978). Many studies have been made to determine what parameters control tropical cyclone genesis and intensification, yet no one process has been shown to be sufficient to explain which tropical systems will intensify. Climatological studies (e.g. Gray, 1979) have indicated the existence of several necessary conditions such as a warm sea surface temperature and a nonzero Coriol is parameter. Other studies have shown that the synoptic scale environment can influence tropical cyclone genesis and intensification. Studies such as those by Hawkins and Rubsam (1968) and Frank and Clark (1979) have shown that tropical cyclones often form from pre-existing synoptic scale disturbances. 
Other studies have shown that upper level weather systems can affect tropical cyclones. For example, Lewis and Jorgensen (1978) presented a case study of the dissipation of a hurricane as it interacted with an upper level trough. Although the influence of the large scale environment has been documented in many observational studies, theoretical and modeling studies in this area have been limited. In Chapter 5 the effect of a horizontally sheared basic state wind in the upper and lower layers on a tropical cyclone simulation is investigated. An example of the interaction of a tropical cyclone with a trough in the upper layer of the model is also presented.

From a forecasting point of view, it is perhaps more important to be able to predict the track of a tropical cyclone rather than its intensity changes. Dynamical models have been used for this purpose for many years and have ranged in complexity from barotropic models (Sanders et a1., 1975) to full primitive equation models with moving nested grids (Hovermale and Livezey, 1977). One problem with using primitive equation models for track forecasting is that the initialization procedure can affect the track of the storm. In Chapter 6, the applicability of nonlinear normal mode initialization to primitive equation track forecasting is investigated. For this purpose, initial data is obtained from a previous model simulation and several initialization procedures are applied. The effect of the initialization is assessed by comparing the model simulations to the simulation where the initial data was not changed. Results from a nondivergent barotropic model are also presented in Chapter 6 to investigate the effect of several model parameters on a tropical cyclone track forecast. 
The relevance of barotropic model track forecasts is evaluated by comparing the barotropic and primitive equation model results. 
CHAPTER 2

GOVERNING EQUATIONS

In this chapter a brief description of the governing equations is presented. The physical model is based on the work of Ooyama (1969a) and the reader is referred to the original paper for further details. Ooyama's model treats the large scale atmosphere as three layers of incompressible fluid with the effects of cumulus convection included as a mass flux between two of the fluid layers. The magnitude of the mass flux is parameterized in terms of the large scale variables. Ooyama's model is generalized by relaxing the axisymmetric and balance approximations and by solving the governing equations on a mid-latitude

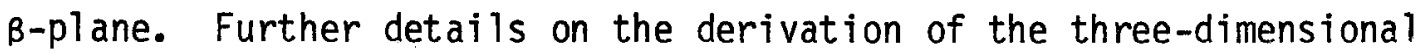
version of Doyama's model can be found in B1 iss (1980).

\subsection{Large-Scale Governing Equations}

Consider a fluid which consists of three stably stratified homogeneous layers as shown in Fig. 1. The equations which govern the motion of this fluid are the momentum and continuity equations for each layer which are given by

$$
\begin{aligned}
& \frac{\partial W_{0}}{\partial t}+f \hat{k} \times V_{0}+\nabla \phi_{0}=-\left(W_{0} \cdot \nabla\right) W_{0}-\frac{W^{-}}{H_{0}}\left(W_{0}-W_{1}\right)+F_{0},(2.1) \\
& \frac{\partial V_{1}}{\partial t}+f \hat{k} \times V_{1}+\nabla \phi_{1}=-\left(W_{1} \cdot \nabla\right) W_{1}+\frac{W^{+}}{\left(H_{1}+h_{1}\right)}\left(W_{0}-W_{1}\right)+I F_{1},
\end{aligned}
$$




$$
\begin{aligned}
\frac{\partial V_{2}}{\partial t}+f \hat{k} \times I V_{2}+\nabla \phi_{2}=-\left(I V_{2} \cdot \nabla\right) I V_{2}+F_{2}, & \\
H_{0} \nabla \cdot I V_{0}+w & =0, \\
\frac{\partial h_{1}}{\partial t}+H_{1} \nabla \cdot I V_{1}-w & =-\nabla \cdot\left(h_{1} \mid V_{1}\right)-Q, \\
\frac{\partial h_{2}}{\partial t}+H_{2} \nabla \cdot I V_{2} & =-\nabla \cdot\left(h_{2} W_{2}\right)+\frac{1}{\varepsilon} Q,
\end{aligned}
$$

where

$$
\begin{aligned}
w_{i} & =u_{i} \hat{i}+v_{i} \hat{j}=\text { horizontal velocity of layer } i(i=0,1,2) \\
u_{i} & =\text { eastward component of horizontal velocity of layer } i \\
v_{i} & =\text { northward component of horizontal velocity of layer } i \\
w & =\text { vertical velocity at the top of layer } 0 \\
H_{i} & =\text { mean thickness of layer } i \\
h_{i} & =\text { deviation of thickness of layer } i \text { from the mean thickness } \\
f & =\text { Coriolis parameter } \\
g & =\text { acceleration of gravity } \\
I_{i} & =\text { friction term of layer } i \\
Q & =\text { mass per unit area and time transported from layer } 1 \text { to layer } 2 \\
\rho_{i} & =\text { density of layer } i \\
\varepsilon & =\rho_{2} / \rho_{1} \\
\phi_{0} & =g\left(h_{1}+\varepsilon h_{2}\right) \\
\phi_{1} & =g\left(h_{1}+\varepsilon h_{2}\right) \\
\phi_{2} & =g\left(h_{1}+h_{2}\right) \\
w^{+} & =1 / 2(|w|+w) \\
w^{-} & =1 / 2(|w|-w) \\
\nabla & =\frac{\partial}{\partial x} \hat{i}+\frac{\partial}{\partial y} \hat{j}
\end{aligned}
$$




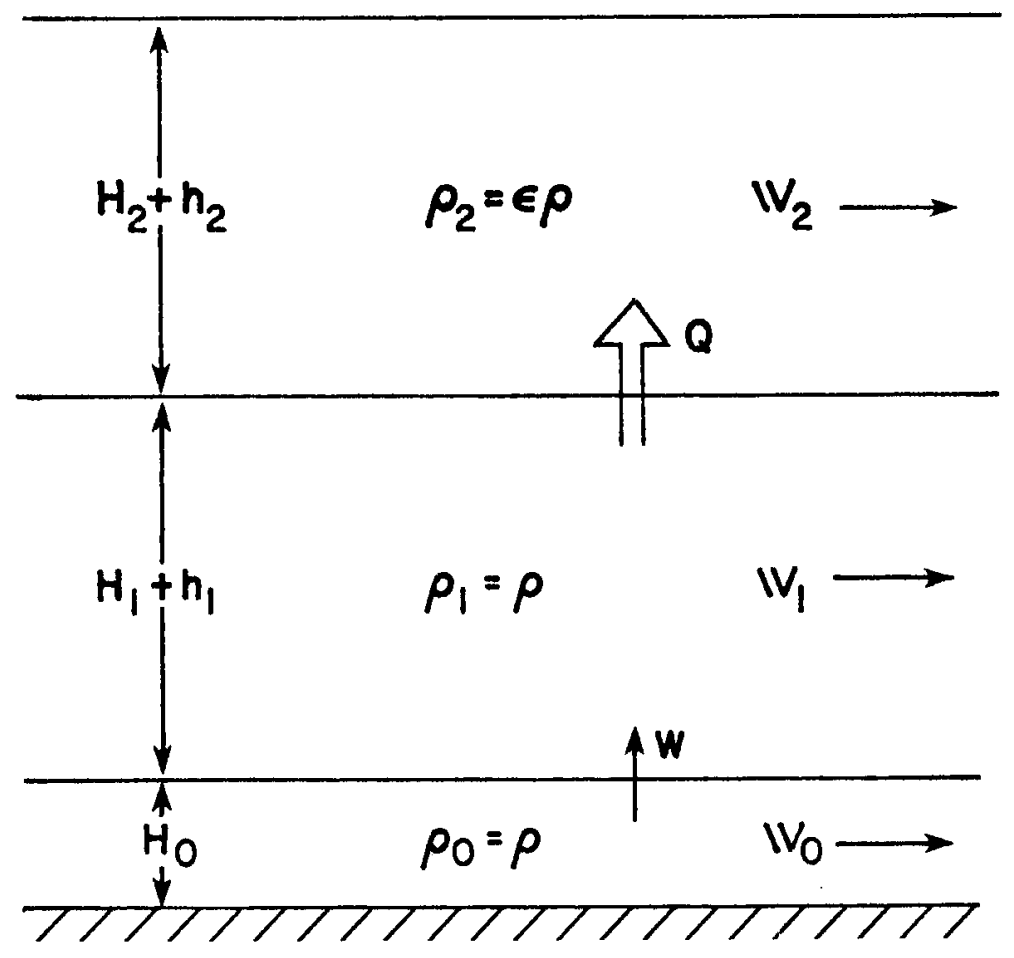

Figure 1. The Ooyama fluid system. 
As can be seen in Fig. 1, variables with subscripts 0,1 or 2 correspond to the lowest, middle or upper layers respectively. Following 0oyama, layer 0 is assumed to have a constant thickness, and the same density as layer 1 . Layer 0 is included so that atmospheric boundary layer processes can be simulated. Since the thickness of layer 0 is constant, the continuity equation (2.4) is simplified and the pressure gradient terms are the same for layers 0 and 1 in (2.1) and (2.2). Since the density of layers 0 and 1 is the same, it is possible for mass to travel across the interface between these layers when $w \neq 0$. The second term from the right in (2.1) and (2.2) must be included so that the total momentum of layers 0 and 1 is conserved when this transport occurs.

The mass transport term $Q$ which appears in (2.5) and (2.6) allows the inclusion of diabatic effects in the incompressible fluid system. In a compressible fluid, diabatic processes allow fluid parcels to move across surfaces of constant potential temperature. In the incompressible system, diabatic processes allow fluid parcels to move between layers of different density. In section 2.3 it will be shown how $Q$ can be diagnosed from the large scale variables to simulate the diabatic effects of cumulus convection.

The remaining terms in (2.1)-(2.6) are fairly conventional. The friction terms in (2.1)-(2.3) are functions of the dependent variables and will be discussed in more detail in the next section. Assuming the friction terms can be calculated and $Q$ can be diagnosed, (2.1)-(2.6) are a closed system of nine scalar equations with independent variables $x, y$ and $t$ and dependent variables $u_{0}, v_{0}, w, u_{1}, v_{1}, u_{2}, v_{2}, h_{1}$ and $h_{2}$. 


\subsection{Specification of Friction Terms}

The friction terms in (2.1)-(2.3) are given by

$$
\begin{array}{ll}
\mathbb{F}_{0}=\frac{-C_{D}}{H_{0}}\left|\mathbb{V}_{0}\right| \mathbb{W}_{0}+\lambda \nabla^{2} \mathbb{W}_{0}, \\
\mathbb{F}_{1}= \\
\mathbb{F}_{2}= & \lambda \nabla^{2} \mathbb{V}_{1}-\mu \frac{\left(\mathbb{V}_{1}-\mathbb{V}_{2}\right)}{\left(H_{1}+h_{1}\right)}, \\
& \lambda \nabla^{2} \mathbb{W}_{2}+\mu \frac{\left(\mathbb{V}_{1}-\mathbb{V}_{2}\right)}{\varepsilon\left(H_{2}+h_{2}\right)}+\frac{Q}{\varepsilon} \frac{\left(\mathbb{V}_{1}-\mathbb{V}_{2}\right)}{\left(H_{2}+h_{2}\right)}
\end{array}
$$

where $\left|V_{0}\right|$ is the magnitude of the boundary layer horizontal velocity. Term A, which appears only in the boundary layer equation, represents the surface drag calculated from the bulk aerodynamic formula. The drag coefficient $C_{D}$ is assumed to have a constant value of 0.0015 . The terms labeled (B) represent horizontal eddy diffusion of momentum where the horizontal eddy diffusion coefficient $\lambda$ is assumed to have a constant value of $10^{3} \mathrm{~m}^{2} \mathrm{~s}^{-1}$. The terms labeled (C) represent the effect of vertical diffusion due to the velocity shear across the interface between layers 1 and 2 . These terms act in the usual downgradient sense (i.e. to reduce the vertical shear) and the shear stress coefficient $\mu$ has a constant value of $5 \times 10^{-4} \mathrm{~ms}^{-1}$. Term (D) represents the effect of the mixing of momentum when mass is transported from layer 1 to layer 2. This term can be derived by considering an amount of mass per unit area $\rho \Delta h$ which is transported from layer 1 to layer 2 in time $\Delta t$. The horizontal velocity of layer 1 is not changed by this process, but the thickness of this layer decreases by an amount $\Delta h$. Assuming this mass conserves the momentum of layer 1 and mixes with the momentum of layer 
2 , the horizontal velocity of layer 2 becomes the mass weighted average of the two velocities which is given by

$$
\mathbb{W}_{2}(t+\Delta t)=\frac{\varepsilon \rho \mathbb{V}_{2}(t)\left(H_{2}+h_{2}\right)+\rho \Delta h \mathbb{W}_{1}(t)}{\varepsilon \rho\left(H_{2}+h_{2}\right)+\rho \Delta h} .
$$

The change of $\mathrm{IV}_{2}$ in time $\Delta t$ then becomes

$$
\frac{\mathbb{W}_{2}(t+\Delta t)-\mathbb{W}_{2}(t)}{\Delta t}=\frac{\Delta h}{\Delta t}\left(\frac{\mathbb{W}_{1}(t)-\mathbb{W}_{2}(t)}{\varepsilon\left(H_{2}+h_{2}\right)+\Delta h}\right) .
$$

In the limit as $\Delta t$ and $\Delta h$ approach zero, the above equation becomes

$$
\frac{d W_{2}}{d t}=\frac{Q}{\varepsilon} \frac{\left(W_{1}-W_{2}\right)}{H_{2}+h_{2}}
$$

\subsection{Cumulus Parameterization}

As discussed in section 2.1, diabatic effects in the incompressible fluid system are represented by a mass flux from layer 1 to layer 2. In order to simulate a tropical cyclone, the diabatic effects of cumulus convection must be included, but the absense of true thermodynamics as well as the coarse vertical resolution of the model prohibit the treatment of individual cumulus clouds. Following 0oyama, the diabatic term Q which represents the collective effects of many cumulus clouds is incorporated into the model. This section summarizes the parameterization of $Q$ in terms of the large scale variables.

If a moist parcel of air is lifted without mixing, its temperature will decrease dry adiabatically until the condensation level is reached. After this point, the temperature of the air parcel will decrease at the moist adiabatic lapse rate. Fig. 2 shows the path of an air parcel on a 


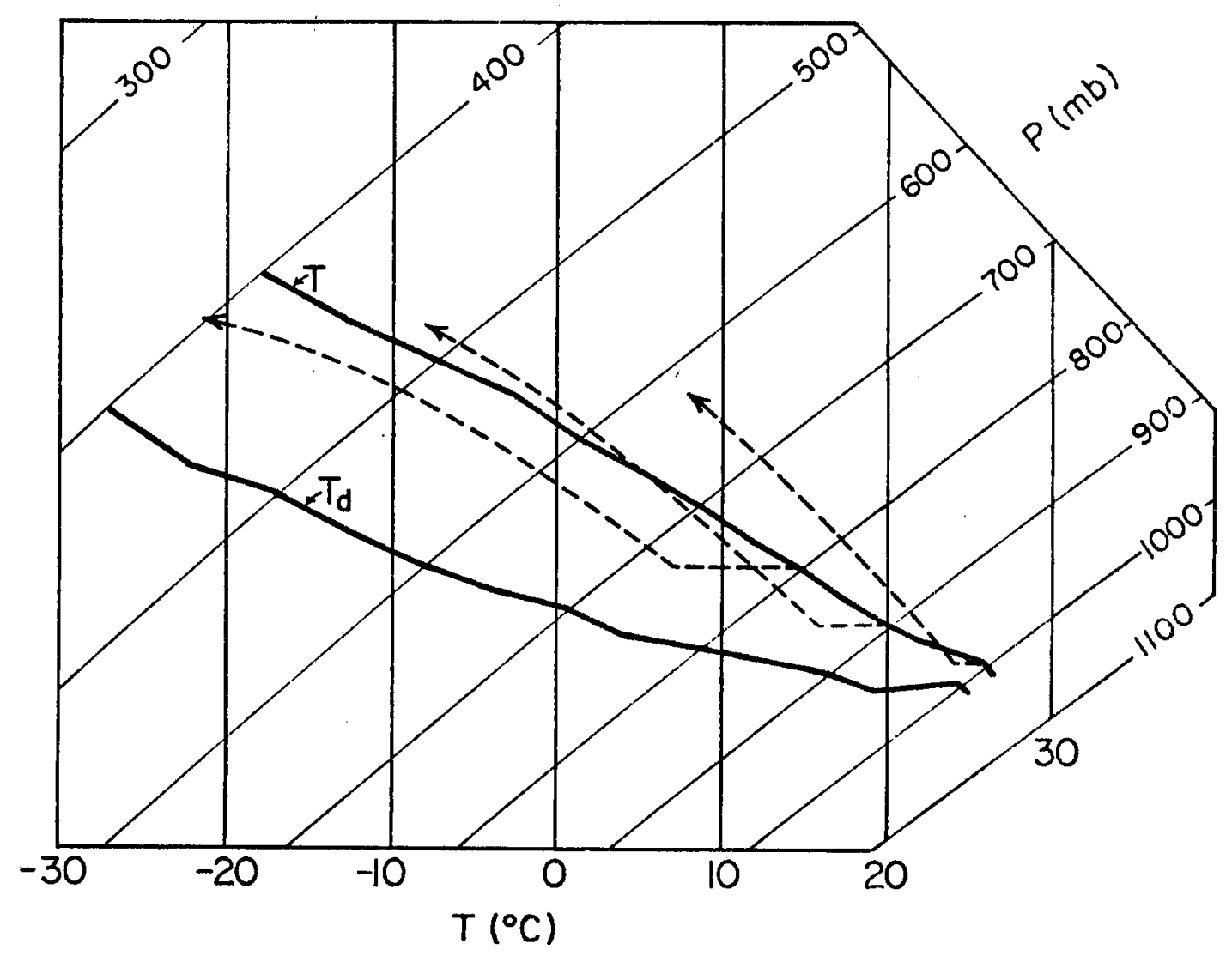

Figure 2. The paths of air parcels (dashed 1ines) on a Tephigram when 1 ifted from 1000,900 and $800 \mathrm{mb}$ for the mean tropical a tmosphere from Jordan (1958). 
thermodynamic diagram when lifted from various levels of the mean tropical atmosphere from Jordan (1958). When the temperature of an air parcel becomes greater than that of the environment it becomes buoyant and will continue to rise. As can be seen in Fig. 2, the only parcels which become buoyant are those which are lifted from below about $900 \mathrm{mb}$. This is caused by the higher temperatures and mixing ratios (as indicated by the higher dewpoint temperatures) in the lower levels. In order to support convective activity for a period of time longer than the time scale of an individual cumulus cloud it is apparently necessary to supply convectively unstable air in the lower levels through convergence. In the context of the three-layer model, it is then assumed that $Q$ is given by

$$
Q=\left\{\begin{array}{ccc}
n w & \text { if } & w>0 \\
0 & \text { if } & w \leq 0
\end{array}\right.
$$

where $\eta$ is a nondimensional constant of proportionality.

When cumulus convection occurs, the rising air does not remain isolated from the surrounding environment as in simple parcel arguments, but rather entrains some of the environmental air. Equation (2.13) indicates that for every unit of boundary layer air that goes up in a convective cloud, $(n-1)$ units are entrained from layer 1 and $n$ units are added to layer 2. The moist static energy $M$ of a unit mass of air is given by

$$
M=c_{p} T+L q+g z
$$

where $c_{p}$ is the specific heat at constant pressure, $T$ is temperature, $L$ is the latent heat of condensation, $q$ is the mixing ratio of water vapor and $\mathrm{gz}$ is the geopotential energy. The moist static energy of the saturated air which enters the upper layer is then the mass weighted 
average of the boundary layer air and the entrained layer 1 air which is given by

$$
M_{2}=\frac{M_{0}+(n-1) M_{1}}{n} \text {, }
$$

where $M_{0}, M_{1}$ and $M_{2}$ are the moist static energy of layers 0,1 and 2 respectively. Solving (2.15) for $n$ gives

$$
n=1+\frac{M_{0}-M_{2}}{M_{2}-M_{1}}
$$

The value of $M_{2}$ in (2.16), which represents the moist static energy of the air inside the cumulus clouds, can be approximated by the moist static energy of the environmental air in layer 2 with the value of $q$ replaced by the saturation value $q_{s}$. This can be done since the cloud air has approximately the same temperature as the environment when it reaches the upper layer, but is saturated.

The differential relation between $M$ and equivalent potential temperature $\theta_{\mathrm{e}}$ is given by

$$
d M=\left(\frac{c_{p}^{\top}}{\theta_{e}}\right) d \theta_{e} \text {. }
$$

Since the term $\frac{c_{p}{ }^{\top}}{\theta_{e}}$ varies slowly, (2.16) can be written in terms of $\theta_{e}$. For convenience let the following definitions be made:

$$
\left.\begin{array}{l}
\Lambda_{0}=\left(\theta_{e}\right)_{0}-\theta \\
\Lambda_{1}=\left(\theta_{e}\right)_{1}-\theta \\
\Lambda_{2}=\left(\theta_{e}\right)_{2}^{*}-\theta
\end{array}\right\},
$$

where $\left(\theta_{e}\right)_{0}$ and $\left(\theta_{e}\right)_{1}$ are the equivalent potential temperatures of 
layers 0 and 1 respectively, $\left(\theta_{e}^{*}\right)_{2}$ is the saturated equivalent potential temperature of layer 2 and $\theta$ is a constant reference temperature. Using ( $\mathbf{c} .18)$, equation (2.16) can then be written as

$$
\eta=1+\frac{\Lambda_{0}-\Lambda_{2}}{\Lambda_{2}-\Lambda_{1}}
$$

In order to calculate $\eta$ using (2.19) it is necessary to calculate the equivalent potential temperature of each layer. Following Doyama, the variations of the mid-level $\theta_{e}$ are neglected and $\Lambda_{1}$ is set to a constant value of $-10 \mathrm{~K}$. Ooyama argued that the variation of $\theta_{1}$ was not of critical importance in the basic dynamics of tropical cyclones and the success of his model tends to confirm this assumption.

As a tropical cyclone forms, the upper levels near the storm center can warm by as much as $15^{\circ} \mathrm{C}$ (e.g. Hawkins and Imbembo, 1976). This upper level warming greatly increases the vertical stability which decreases the strength of the deep convection. In order to include this effect in the incompressible fluid system, it is necessary to develop the diagnosis of $\Lambda_{2}$ from an analogous compressible system. The hydrostatic equation for a compressible fluid can be written as

$$
\frac{\partial \phi}{\partial \pi}=-c_{p} \theta,
$$

where $\phi$ is the geopotential, $\theta$ is potential temperature and $\pi$ is defined by $(p / 1000)^{K}$ where $K=R / c_{p}, P$ is the pressure in $n b$ and $R$ is the gas constant for dry air. Integrating (2.20) from $\phi_{1}$ to $\phi_{2}$ gives

$$
\frac{\phi_{2}-\phi_{1}}{\pi_{1}-\pi_{2}}=c_{p} \theta_{m}
$$

where $\theta_{\mathrm{m}}$ is the mean potential temperature between $\phi_{1}$ and $\phi_{2}$. Equation (2.21) can be written as 


$$
\frac{\phi_{2}^{\prime}-\phi_{1}^{\prime}}{\pi_{1}-\pi_{2}}=c_{p}\left(\theta_{m}-\bar{\theta}_{m}\right),
$$

where $\bar{\theta}_{\mathrm{m}}$ is the horizontal average of $\theta_{\mathrm{m}}$ and $\phi^{\prime}$ represents the deviation from the horizontal average of $\phi$. 0oyama noted that the saturated equivalent potential temperature at $300 \mathrm{mb}$ varies from about 340 to $370 \mathrm{~K}$ as the mean potential temperature between $700 \mathrm{mb}$ and $300 \mathrm{mb}$ varies from 324 to $339 \mathrm{~K}$. Assuming level 1 and level 2 represent $700 \mathrm{mb}$ and $300 \mathrm{mb}$ respectively, and assuming a simple linear relationship between $\theta_{\mathrm{e} 2}{ }^{*}$ and $\theta_{\mathrm{m}}$ gives

$$
\theta_{e} 2^{*}-\bar{\theta}_{e} 2^{*}=2.0\left(\theta_{m}-\bar{\theta}_{m}\right)
$$

or

$$
\Lambda_{2}=\bar{\Lambda}_{2}+2.0\left(\theta_{m}-\bar{\sigma}_{m}\right)
$$

Eliminating $\theta_{\mathrm{m}}-\bar{\theta}_{\mathrm{m}}$ between (2.22) and (2.23) gives

$$
\Lambda_{2}=\bar{\Lambda}_{2}+\frac{2.0}{c_{p}\left(\pi_{1}-\pi_{2}\right)}\left(\phi_{2}^{\prime}-\phi_{1}^{\prime}\right)
$$

Equation (2.24) is strictly valid onty for a compressible fluid. The analogy with the incompressible system is made by considering the similar roles of $\phi_{2}^{\prime}$ and $\phi_{1}^{\prime}$ in the compressible system and $\phi_{1}$ and $\phi_{2}$ which appear in (2.1)-(2.3). Using this analogy and the definitions of $\phi_{1}$ and $\phi_{2},(2.24)$ can be written as

$$
\Lambda_{2}=\bar{\Lambda}_{2}+\frac{g \gamma}{c_{p}}(1-\varepsilon) h_{2}
$$

where again it was assumed that layers 1 and 2 correspond to 700 and 300 mb so that $\pi_{1}-\pi_{2}$ could be calculated. This gives the nondimensional constant $\gamma$ in (2.25) a value of 10.3. As $h_{2}$ increases in (2.25), $\Lambda_{2}$ 
increases so that $\eta$ in (2.19) and the magnitude of $Q$ in (2.13) decrease. Thus, the use of (2.25) to diagnose $\Lambda_{2}$ simulates the effect of upper level warming on convection using the analogy between the increase in thickness of an incompressible fluid layer and the increase in temperature of a compressible fluid layer.

The remaining thermodynamic variable necessary for the calculation of $Q$ is the boundary layer equivalent potential temperature deviation $\Lambda_{0}$. Again following Ooyama, $\Lambda_{0}$ is treated as a prognostic variable and is predicted from the conservation equation given by

$$
\frac{\partial \Lambda_{0}}{\partial t}=-u_{0} \frac{\partial \Lambda_{0}}{\partial x}-v_{0} \frac{\partial \Lambda_{0}}{\partial y}-\frac{W^{-}}{H_{0}}\left(\Lambda_{0}-\Lambda_{1}\right)+\lambda \nabla^{2} \Lambda_{0}+\frac{C_{E}}{H_{0}}\left|W_{0}\right|\left(\Lambda_{S}-\Lambda_{0}\right) .
$$

The first two terms on the right side of $(2.26)$ represent horizontal advection and the third term represents vertical advection. The fourth term represents diffusion where the horizontal eddy diffusion coefficient $\lambda$ is assumed to be the same as the coefficient which appears in the momentum diffusion terms in (2.7)-(2.9). The last term on the right side of (2.26) represents the surface flux of equivalent potential temperature as calculated from the bulk derodynamic formula, where $\Lambda_{s}$ is the sea-surface saturation equivalent potential temperature deviation and $C_{E}$ is the air-sea exchange coefficient. The value of $C_{E}$ is assumed to be equal to the exchange coefficient for momentum $C_{D}$ which appears in (2.7).

The saturation equivalent potential temperature of the sea surface is given by

$$
\theta_{e s}^{*}=\theta_{s} \exp \left(\frac{L q_{s}}{c_{p} T_{s}}\right) \text {, }
$$


where $\theta_{S}$ is the potential temperature at the surface pressure $P_{S}$ and sea surface temperature $T_{S}, q_{S}$ is the saturation mixing ratio at $T_{S}, P_{S}$ and $L$ is the latent heat of condensation. In order to allow $\theta$ es to depend on the surface pressure, $\theta_{\text {es }}{ }^{*}$ is expanded in a Taylor series at a fixed value of $T_{S}$ which gives

$$
\left.\theta_{e s}^{*} \cong \theta_{e s}^{*}\right|_{\bar{P}_{S}, T_{S}}+\left.\frac{\partial \theta_{e s}^{*}}{\partial P_{S}}\right|_{\bar{P}_{S}, T_{S}}\left(P_{S}-\bar{P}_{S}\right) \text {. }
$$

In the incompressible fluid system, the deviation of the surface pressure from the mean value is given by $\rho g\left(h_{1}+\varepsilon h_{2}\right)$. Evaluating the right side of $(2.28)$ at $T_{S}=28^{\circ} \mathrm{C}$ and $P_{S}=1015 \mathrm{mb}$, substituting for $\left(P_{S}-\right.$ $\overline{\mathrm{P}}_{\mathrm{s}}$ ) and subtracting $\theta$ from both sides gives

$$
\Lambda_{s}=\bar{\Lambda}_{s}-\frac{g \alpha}{c_{p}}\left(h_{1}+\varepsilon h_{2}\right)
$$

where the constant $\alpha$ is equal to about 1.87. Since the mean surface pressure $P_{S}$ is constant, $\bar{\Lambda}_{S}$ is a function of $T_{S} \cdot$ In all of the model simulations $\bar{\Lambda}_{S}$ is set to $30 \mathrm{~K}$ which corresponds to a sea surface temperature of about $28^{\circ} \mathrm{C}$.

In summary, the diabatic term $Q$ is given by (2.13) where $n$ is given by (2.19). The parameter $n$ is a function of $\Lambda_{0}, \Lambda_{1}$ and $\Lambda_{2}$ which represent the equivalent potential temperature deviations of each of the fluid layers where $\Lambda_{2}$ is diagnosed using (2.25), $\Lambda_{1}$ is assumed to be constant and $\Lambda_{0}$ is predicted using (2.26).

\subsection{Equations of Motion on a Mid-Latitude $\beta$-plane}

Since many early tropical cyclone models were axisymmetric, it was necessary to neglect the variation of the Coriolis parameter $f$ with latitude. As three-dimensional models were developed it became possible 
to allow $f$ to vary with latitude. Several authors (e.g. Madala and Piacsek, 1975; Kitade, 1980) have shown that when $f$ varies with latitude, a model tropical cyclone will no longer remain stationary but will drift to the northwest at speeds between 1 and $3 \mathrm{~ms}^{-1}$. Anthes and Hoke (1975) have shown that the variation of $f$ with latitude can cause a tropical cyclone to become asymmetric with a tendency for confluence to the east and diffluence to the west of the storm center in the low leve1s.

The simplest way to include the variation of $f$ with latitude in a model with cartesian geometry is to make the $\beta$-plane approximation. This approximation assumes $f$ is given by a two term Taylor series of the form

$$
f \cong f_{0}+\beta\left(y-y_{0}\right)
$$

where

$$
\beta=\left.\frac{\partial f}{\partial y}\right|_{y=y_{0}},
$$

and

$$
y=a \theta, \quad y_{0}=a \theta_{0},
$$

where $\theta$ is latitude, $a$ is the radius of the earth and $\theta_{0}$ is the reference latitude. As discussed by Lindzen (1967), there are two types of B-plane approximations which can be made. The first of these is the equatorial $\beta-p l a n e$ where the reference latitude $\theta_{0}$ is assumed to be the equator and $f$ is equal to $B y$. The second of these is the mid-latitude $\beta-p l a n e$ where $\theta_{0}$ is assumed to be some latitude away from the equator. For the mid-latitude $\beta-p l a n e$ it is necessary to neglect By compared to $f_{0}$, except where $f$ is differentiated. In the current model, it will be assumed that each of the dependent variables is periodic in the $x$ and $y$ 
directions so that they can be expanded in Fourier series. If the equatorial $\beta-p l a n e$ is used, the equations of motion will contain $\beta y$ terms which will not be periodic in the north-south direction. If the mid-latitude $\beta-p l$ ane is used, however, the only additional terms which appear will be proportional to $f_{0}$ or $\beta$ since $\beta y$ is always neglected compared to $f_{0}$. Thus, the only terms in the governing equations which are related to the variation of $f$ with latitude will have constant coefficients. If the initial values of the dependent variables are specified to be periodic in $x$ and $y$, then the dependent variables will remain periodic for this case. For this reason, the mid-latitude $\beta$ plane can be used with a domain which is periodic in the north-south direction.

In order to use the mid-latitude $\beta$-plane approximation it is necessary to use the differentiated form of the momentum equations. For the differentiated momentum equations, the dependent variables $u_{i}$ and $v_{i}$ are replaced by the vorticity $\zeta_{i}$ and divergence $\delta_{i}$ where

$$
\left.\begin{array}{l}
\zeta_{i}=\frac{\partial v_{i}}{\partial x}-\frac{\partial u_{i}}{\partial y} \\
\delta_{i}=\frac{\partial u_{i}}{\partial x}+\frac{\partial v_{i}}{\partial y}
\end{array}\right\},
$$

and the subscript $i$ refers to layer 0,1 or 2 . The prognostic equation for $\zeta_{i}$ can be derived by taking $\frac{\partial}{\partial x}$ of the $v_{i}$ momentum equation and subtracting $\frac{\partial}{\partial y}$ of the $u_{i}$ momentum equation. Using (2.1)-(2.3), (2.30) and neglecting $\beta y$ compared to $f_{0}$ except where $f$ is differentiated gives 


$$
\begin{aligned}
\frac{\partial \zeta_{i}}{\partial t}+f_{0} \delta_{i}+\beta v_{i} & = \\
& -\frac{\partial}{\partial x}\left(u_{i} \zeta_{j}\right)-\frac{\partial}{\partial y}\left(v_{i} \zeta_{i}\right)+\frac{\partial}{\partial x}\left(v_{y i}+F_{y i}\right)-\frac{\partial}{\partial y}\left(v_{x i}+F_{x i}\right),
\end{aligned}
$$

where

$$
\begin{array}{ll}
v_{x 0}=\frac{-w^{-}}{H_{0}}\left(u_{0}-u_{1}\right), & v_{y 0}=\frac{-w^{\prime}}{H_{0}}\left(v_{0}-v_{1}\right), \\
v_{x 1}=\frac{w^{+}}{\left(H_{1}+h_{1}\right)}\left(u_{0}-u_{1}\right), & v_{y 1}=\frac{w^{+}}{\left(H_{1}+h_{1}\right)}\left(v_{0}-v_{1}\right), \\
v_{x 2}=0, & v_{y 2}=0,
\end{array}
$$

and $F_{x i}$ and $F_{y i}$ are the $x$ and $y$ components of $\mid F_{j}$ defined in (2.7)(2.9). Similarly, the prognostic equation for $\delta_{i}$ can be derived by taking $\frac{\partial}{\partial x}$ of the equation for $u_{i}$ and adding it to $\frac{\partial}{\partial y}$ of the equation for $v_{i}$ and again neglecting $\beta y$ compared to $f_{0}$ except where $f$ is differentiated which gives

$$
\frac{\partial \delta_{i}}{\partial t}-f_{0} \zeta_{i}+\beta u_{i}+\nabla^{2} \phi_{i}=
$$

$-\frac{\partial}{\partial y}\left(u_{i} \zeta_{i}\right)+\frac{\partial}{\partial x}\left(v_{i} \zeta_{i}\right)-\nabla^{2}\left(\frac{u_{i}^{2}+v_{i}^{2}}{2}\right)+\frac{\partial}{\partial x}\left(F_{x i}+v_{x i}\right)+\frac{\partial}{\partial y}\left(F_{y i}+v_{y i}\right)$.

In order to use (2.32) and (2.33) in place of (2.1)-(2.3) it is necessary to relate the velocity components $u_{i}$ and $v_{i}$ to $\zeta_{i}$ and $\delta_{i}$. This is accomplished by defining a streamfunction $\psi_{j}$ and velocity potential $x_{i}$ for each layer as follows:

$$
\left.\begin{array}{l}
u_{i}=-\frac{\partial \psi_{i}}{\partial y}+\frac{\partial x_{i}}{\partial x} \\
v_{i}=\frac{\partial \psi_{i}}{\partial x}+\frac{\partial x_{i}}{\partial y}
\end{array}\right\} .
$$


Substituting the above equations into (2.31) gives

$$
\left.\begin{array}{l}
\zeta_{i}=\nabla^{2} \psi_{i} \\
\delta_{i}=\nabla^{2} x_{i}
\end{array}\right\} .
$$

If $\psi_{i}$ and $x_{i}$ are considered the unknowns, then (2.32) and (2.33) for $i=$ $0,1,2$ are six equations which can be used in place of the six scalar momentum equations (2.1)-(2.3).

In the linear case, all the terms on the right side of (2.32) and (2.33) are neglected. These equations can be combined with linearized forms of the continuity equations $(2.4)-(2.6)$ to give a system of nine equations in nine unknowns. In the linear case the forcing and friction terms are neglected so that solutions should be oscillatory in time. Stevens et al. (1977) have shown that this will be true only if the velocity potential contribution to the $\beta v_{j}$ term in (2.32) and the streamfunction contribution to the $\mathrm{Bu}_{i}$ term in (2.33) are neglected. Equations (2.32) and (2.33) then become

$$
\begin{aligned}
& \frac{\partial \zeta_{i}}{\partial t}+f_{0} \delta_{i}+\beta \frac{\partial \psi_{i}}{\partial x}= \\
& -\frac{\partial}{\partial x}\left(u_{i} \zeta_{i}\right)-\frac{\partial}{\partial y}\left(v_{i} \zeta_{j}\right)+\frac{\partial}{\partial x}\left(v_{y i}+F_{y i}\right)-\frac{\partial}{\partial y}\left(v_{x i}+F_{x i}\right), \quad \text { (2.36) } \\
& \quad \frac{\partial \delta_{i}}{\partial t}-f_{0} \zeta_{i}+\beta \frac{\partial x_{i}}{\partial x}+\nabla^{2} \phi_{i}= \\
& -\frac{\partial}{\partial y}\left(u_{i} \zeta_{j}\right)+\frac{\partial}{\partial x}\left(v_{i} \zeta_{j}\right)+\frac{\partial}{\partial x}\left(v_{x i}+F_{x i}\right)+\frac{\partial}{\partial y}\left(v_{y i}+F_{y i}\right)-\nabla^{2}\left(\frac{u_{i}^{2}+v_{i}{ }^{2}}{2}\right) .
\end{aligned}
$$

It will be shown in chapter 3 that the eigenfrequencies of the linear 
equations correspond to the usual mid-latitude $\beta$-plane Rossby waves or inertia-gravity waves.

\subsection{Summary of the Governing Equations}

For convenience, the complete system of equations for the threedimensional version of 0oyama's model on a mid-latitude $\beta$-plane are listed below:

$$
\begin{aligned}
& \frac{\partial \zeta_{i}}{\partial t}+f_{0} \delta_{i}+\beta \frac{\partial \psi_{i}}{\partial x}= \\
& -\frac{\partial}{\partial x}\left(u_{i} \zeta_{j}\right)-\frac{\partial}{\partial y}\left(v_{i} \zeta_{i}\right)+\frac{\partial}{\partial x}\left(v_{y i}+F_{y i}\right)-\frac{\partial}{\partial y}\left(v_{x i}+F_{x i}\right), \\
& \frac{\partial \delta_{i}}{\partial t}-f_{0} \zeta_{i}+\beta \frac{\partial x_{i}}{\partial x}+\nabla^{2} \phi_{i}= \\
& -\frac{\partial}{\partial y}\left(u_{i} \zeta_{j}\right)+\frac{\partial}{\partial x}\left(v_{i} \zeta_{i}\right)+\frac{\partial}{\partial y}\left(v_{y i}+F_{y i}\right)+\frac{\partial}{\partial x}\left(v_{x i}+F_{x i}\right)-\nabla^{2}\left(\frac{u_{i}^{2}+v_{i}^{2}}{2}\right), \\
& H_{0} \delta_{0}+w=0, \\
& \frac{\partial h_{1}}{\partial t}+H_{1} \delta_{1}-w=-\frac{\partial}{\partial x}\left(h_{1} u_{1}\right)-\frac{\partial}{\partial y}\left(h_{1} v_{1}\right)-Q \\
& \frac{\partial h_{2}}{\partial t}+H_{2} \delta_{2}=-\frac{\partial}{\partial x}\left(h_{2} u_{2}\right)-\frac{\partial}{\partial y}\left(h_{2} v_{2}\right)+\frac{1}{\varepsilon} Q \\
& Q=n w^{+}, \\
& n=\frac{\Lambda_{0}-\Lambda_{2}}{\Lambda_{2}-\Lambda_{1}},
\end{aligned}
$$


28

$$
\begin{aligned}
& \Lambda_{2}=\bar{\Lambda}_{2}+\frac{g \gamma}{c_{p}}(1-\varepsilon) h_{2}, \\
& \Lambda_{1}=-10 \mathrm{~K} \text {, } \\
& \frac{\partial \Lambda_{0}}{\partial t}=-u_{0} \frac{\partial \Lambda_{0}}{\partial x}-v_{0} \frac{\partial \Lambda_{0}}{\partial y}-\frac{W^{-}}{H_{0}}\left(\Lambda_{0}-\Lambda_{1}\right)+\lambda \nabla^{2} \Lambda_{0}+\frac{C_{E}}{H_{0}}\left|V_{0}\right|\left(\Lambda_{S}-\Lambda_{0}\right), \quad \text { (2.47) } \\
& \Lambda_{s}=\bar{\Lambda}_{s}-\frac{g \alpha}{c_{p}}\left(h_{1}+\varepsilon h_{2}\right), \\
& \zeta_{i}=\nabla^{2} \psi_{i}, \quad \delta_{i}=\nabla^{2} \chi_{j}, \\
& u_{i}=-\frac{\partial \psi_{i}}{\partial y}+\frac{\partial x_{i}}{\partial x}, \quad v_{i}=\frac{\partial \psi_{i}}{\partial x}+\frac{\partial x_{i}}{\partial y}, \\
& \phi_{0}=\phi_{1}=g\left(h_{1}+\varepsilon h_{2}\right) \text {, } \\
& \phi_{2}=g\left(h_{1}+h_{2}\right) \text {, } \\
& v_{x_{0}}=-\frac{W^{-}}{H_{0}}\left(u_{0}-u_{1}\right), \quad v_{y_{0}}=-\frac{W^{-}}{H_{0}}\left(v_{0}-v_{1}\right), \\
& v_{x 1}=\frac{w^{+}}{\left(H_{1}+h_{1}\right)}\left(u_{0}-u_{1}\right), \quad v_{y 1}=\frac{w^{+}}{\left(H_{1}+h_{1}\right)}\left(v_{0}-v_{1}\right), \\
& v_{x 2}=0, \quad v_{y 2}=0 \text {, } \\
& F_{x 0}=-\frac{C_{D}}{H_{0}}\left|V_{0}\right| u_{0}+\lambda \nabla^{2} u_{0}, \quad F_{y o}=-\frac{C_{D}}{H_{0}}\left|v_{0}\right| v_{0}+\lambda \nabla^{2} v_{0}, \\
& F_{x 1}=-\mu \frac{\left(u_{1}-u_{2}\right)}{\left(H_{1}+h_{1}\right)}+\lambda \nabla^{2} u_{1}, \quad F_{y 1}=-\mu \frac{\left(v_{1}-v_{2}\right)}{\left(H_{1}+h_{1}\right)}+\lambda \nabla^{2} v_{1}, \quad \text { (2.57) }
\end{aligned}
$$




$$
\left.\begin{array}{l}
F_{x 2}=\mu \frac{\left(u_{1}-u_{2}\right)}{\varepsilon\left(H_{2}+h_{2}\right)}+\lambda \nabla^{2} u_{2}+\frac{Q}{\varepsilon} \frac{\left(u_{1}-u_{2}\right)}{\left(H_{2}+h_{2}\right)} \\
F_{y 2}=\mu \frac{\left(v_{1}-v_{2}\right)}{\varepsilon\left(H_{2}+h_{2}\right)}+\lambda \nabla^{2} v_{2}+\frac{Q}{\varepsilon} \frac{\left(v_{1}-v_{2}\right)}{\left(H_{2}+h_{2}\right)}
\end{array}\right\},
$$

Table 1 lists the prognostic and diagnostic variables which appear in the above equations and gives the values of the specified parameters which were used in all of the model integrations. The value of the sea surface temperature is $28^{\circ} \mathrm{C}$ as discussed previously and $f_{0}$ and $\beta$ are evaluated at $20^{\circ} \mathrm{N}$. 
TABLE 1.

Summary of Dependent Variables and Related Parameters.

\begin{tabular}{|c|c|c|c|}
\hline $\begin{array}{l}\text { Prognostic } \\
\text { Variables }\end{array}$ & $\begin{array}{l}\text { Diagnostic } \\
\text { Variables }\end{array}$ & $\begin{array}{l}\text { Specified } \\
\text { Parameters }\end{array}$ & $\begin{array}{l}\text { Equations where } \\
\text { parameters appear }\end{array}$ \\
\hline$\psi_{i}$ & $\zeta_{j}$ & $\mathrm{H}_{0}=1 \mathrm{~km}$ & several \\
\hline$x_{i}$ & $\delta_{i}$ & $H_{1}=H_{2}=5 \mathrm{~km}$ & several \\
\hline$h_{1}$ & $u_{i}$ & $\varepsilon=0.9$ & several \\
\hline$h_{2}$ & $v_{i}$ & $\left.f_{0}\right)$ values & $(2.38),(2.39)$ \\
\hline \multirow[t]{12}{*}{$\Lambda_{0}$} & w & B $\int$ at $20^{\circ} \mathrm{N}$ & $(2.38),(2.39)$ \\
\hline & $n$ & $\mathrm{~g}=9.8 \mathrm{~ms}^{-2}$ & several \\
\hline & Q & $c_{p}=1004 \mathrm{~J} \mathrm{deg}^{-1} \mathrm{~kg}^{-1}$ & $(2.45),(2.48)$ \\
\hline & $\Lambda_{2}$ & $\bar{\Lambda}_{2}=0 \mathrm{~K}$ & $(2.45)$ \\
\hline & $\Lambda_{S}$ & $\Lambda_{1}=-10 \mathrm{~K}$ & $(2.46)$ \\
\hline & $\phi_{i}$ & $\bar{\Lambda}_{\mathrm{S}}=30 \mathrm{~K}$ & $(2.48)$ \\
\hline & & $\gamma=10.3$ & $(2.45)$ \\
\hline & & $\alpha=1.87$ & $(2.48)$ \\
\hline & & $C_{D}=0.0015$ & $(2.56)$ \\
\hline & & $C_{E}=0.0015$ & $(2.47)$ \\
\hline & & $\lambda=10^{3} \mathrm{~m}^{2} \mathrm{~s}^{-1}$ & severa 1 \\
\hline & & $\mu=5 \times 10^{-4} \mathrm{~m} \mathrm{~s}^{-1}$ & $(2.57),(2.58)$ \\
\hline
\end{tabular}




\section{CHAPTER 3}

\section{SOLUTION USING THE FOURIER-GALERKIN METHOD}

As discussed in chapter 1 , all of the tropical cyclone models to date have been solved using finite difference methods. Spectral methods have been shown to have many computational advantages over finite difference methods including much greater accuracy per degree of freedom, reduction of computational dispersion and elimination of nonlinear instability (Gottlieb and Orszag, 1977). In order to gain these computational advantages, a spectral method will be used to solve the governing equations developed in chapter 2.

In the meteorological literature, the term spectral method has a fatrly specific meaning, but as discussed by Gottlieb and Orszag (1977), this term can refer to one of three methods known as the Galerkin, tau and collocation methods. For each of these methods, the spatial dependence of the dependent variables is expanded in a finite series of some appropriate basis functions. The governing equations are then used to give a system of equations for the series amplitudes. The differences between these methods are the way in which boundary conditions are treated and the way the equations for the series amplitudes are determined.

For the Galerk in method, the bas is functions are chosen so that they satisfy the same boundary conditions as the dependent variables and are orthogonal with respect to some inner product. The equations for the time dependent series amplitudes are then found by substituting the 
series expansions into the governing equations and taking the inner product of each equation with each of the basis functions. In meteorology, this method has been used extensively for problems in spherical geometry with either spherical harmonics or Hough functions as basis functions (e.g., Hoskins and Simmons, 1975; Kasahara 1977, 1978).

The tau method is a variation of the Galerkin method where the basis functions are still orthogonal, but are not required to satisfy the boundary conditions individually. Instead, extra degrees of freedom are added in such a way that the series as a whole satisfies the boundary conditions. With the exception of the extra terms in the series expansions, the time dependent series amplitudes are determined in the same way as for the Galerkin method. The tau method is generally used for problems with limited area domains and general boundary conditions and the appropriate basis functions for this case are Chebyshev polynomials.

For collocation (also referred to as the psuedospectral method), the equations for the time dependent series amplitudes are determined by substituting the series expansions into the governing equations and then forcing the equations to be satisfied exactly on a set of grid points (collocation points), where the number of collocation points is chosen to be equal to the number terms in the series expansions. For collocation, the equations are solved in physical space, while for the Galerkin and tau methods, the equations are solved in spectral space.

Each of the three spectral methods described above could be used to solve the governing equations derived in chapter 2. At first glance, the tau method appears to be the best choice since it allows the inclusion of a radiation type boundary condition. As mentioned in 
chapter 1 , however, the Chebyshev polynomials oscillate rapidly near the boundaries. This extra boundary resolution makes it necessary to use a very small time step or use some type of implicit time differencing scheme. The simplest type of implicit scheme which could be used is the semi-implicit method where only the linear terms are treated implicitly. Semi-implicit methods have been used in finite difference models (Kwizak and Robert, 1971) to reduce the stability criterion required by the gravity wave part of the solution. When the semi-implicit method is applied in a finite difference model, an elliptic equation must be solved at each time step. The elliptic equation which appears can be solved fairly easily using relaxation methods or by direct elimination. When the semi-implicit method is applied using the tau method with Chebyshev basis functions, the system which must be solved is considerably more complicated. Basically, this is because in a secondorder finite difference model, for example, derivative operators couple the value of a dependent variable at a grid point only to the values at the neighboring grid points. For the tau method with Chebyshev basis functions, however, derivative operators couple all of the series amplitudes, so the linear system which results from the implicit terms contains a full matrix. It may be possible to overcome this difficulty by solving the linear system using an interative procedure. Haidvogel et al. (1980) used a scheme based on the alternating direction implicit (ADI) method in a balanced barotropic ocean model solved with the tau method where the advection terms on the boundaries were treated implicitly. The above procedure would be more difficult in the current model since the terms which govern the motion of the gravity waves would also have to be treated implicitly. 
In order to keep the current model tractable, the Galerkin method was chosen over the tau method. For the Galerkin method the basis functions must satisfy the same boundary conditions as the dependent variables. For this reason it is not possible to include a radiation type boundary condition in the model. Instead, an infinite domain is simulated by using a domain which is periodic in the $x$ and $y$ directions, but is relatively large compared to the size of the disturbance being studied. For this case, the appropriate basis functions are Fourier components and the series expansions are truncated double Fourier series. This reduces the need for the use of implicit time differencing since the Fourier components have uniform resolution over the domain. It turns out, however, that when fourier components are used as basis functions, semi-implicit time differencing can be implemented quite easily since the Fourier components are the eigenfunctions of the 1 inear operators which appear. It is then not necessary to solve a linear system at each time step since all the series amplitudes which appear in the linear terms are decoupled. In fact, in the current model, the linear terms can be calculated exactly, as will be shown in section 3.4.

The governing equations in chapter 2 could also be solved using collocation with the Fourier components as basis functions without encountering the numerical difficulties discussed for the tau method. As mentioned previously, the governing equations are solved in physical space for the collocation method, and in spectral space for the Galerkin method. Since the Fourier components are the eigenfunctions of the linear operators which appear in the governing equations, the linear terms have a very simple form in spectral space. For this reason the Galerkin method was chosen over collocation. 
A possible objection to the use of a doubly-periodic domain in a tropical cyclone model is that the gravity wave energy which is generated by the forcing cannot leave the domain. The linear theory of geostrophic adjustment (e.g. Schubert et al., 1980) indicates that when an impulsive heat source is applied in low latitudes, most of the forcing generates gravity wave motion and only a small portion generates geostrophically balanced flow. These results can be quite misleading, however, since they are based on initial value problems where all the heat is added instantaneously. Silva Dias et al. (1983) have shown in a linear model on a equatorial B-plane, that when the heating occurs over a finite amount of time which is longer than the period of the gravity wave oscillations, considerably less gravity wave energy is generated. In the current model the diabatic heating is parameterized in terms of the large scale variables so it should generally change slowly in time compared to the period of the gravity waves. As will be shown in section 3.3, the amplitude of the gravity wave portion of the total solution can be calculated explicitly. In chapter 4 it will be shown that the amount of energy in the gravity wave part of the solution is more than an order of magnitude smaller than the amount of energy in the balanced part of the solution during a typical model run.

\subsection{Spectral Form of the Governing Equations}

In this section the Galerkin method is applied to give an approximate solution to the governing equations derived in chapter 2. In order to illustrate the method, a simple example is presented. Consider the following equation on the $x$ domain $(a, b)$ : 


$$
\frac{\partial u}{\partial t}+L u=0
$$

where $u=u(x, t)$ and $L$ is some differential operator which may be nonlinear. To solve (3.1) using the Galerkin method, approximate $u(x, t)$ by a finite series of the form

$$
u(x, t) \cong \bar{u}(x, t)=\sum_{j=1}^{N} \hat{u}_{j}(t) \xi_{j}(x)
$$

where it is assumed that the basis functions $\xi_{j}(x)$ satisfy the same boundary conditions as $u(x, t)$ and are orthonormal with respect to the inner product defined by

$$
(u, v)=\int_{a}^{b} u v^{*} d x
$$

where ()$^{*}$ indicates complex conjugate. The inner product of $\xi_{i}(x)$ with $\xi_{j}(x)$ is then given by

$$
\left(\xi_{i}, \xi_{j}\right)=\delta_{i j}
$$

where $\delta_{i j}$ is the Kronecker delta. Substituting (3.2) into (3.1) and taking the inner product with $\xi_{j}(x)$ gives

$$
\frac{\hat{d u}_{j}}{d t}+\left(L\left[\sum_{i=1}^{N} \hat{u}_{j} \xi_{j}\right], \xi_{j}\right)=0 \quad j=1,2 \cdots N
$$

where (3.4) was used to eliminate all but the $\hat{u}_{j}$ term in the time derivative. Equation (3.5) then represents $N$ ordinary differential equations which can be solved using time differencing for the series amplitudes. 
The Galerkin method is based on an orthogonality principle which can be seen by considering (3.5) in the following form:

$$
\left(\frac{\partial \bar{u}}{\partial t}+L \bar{u}, \xi_{j}\right)=0 \quad j=1,2 \cdots N
$$

As $N \rightarrow \infty,(3.6)$ implies that $\frac{\partial \bar{u}}{\partial t}+L \bar{u}$ is orthogonal to an infinite set of the basis functions. Assuming the basis functions form a complete set, then $\frac{\partial \bar{u}}{\partial t}+L \bar{u}$ must be zero since the only vector which can be arthogonal to all the elements of a complete set is the zero vector. When $N$ is finite, then $\frac{\partial \bar{u}}{\partial t}+L \bar{u}$ is approximately zero.

For the governing equations it is assumed that all of the dependent variables are periodic on the $x$ interval $\left[0, L_{x}\right]$ and the $y$ interval $\left[0, L_{y}\right]$. Each of the dependent variables can then be expanded in truncated double Fourier series given by

$$
\begin{aligned}
& F(x, y, t)=\sum_{k \ell} \sum_{k \ell} \hat{F}_{k \ell}(t) \xi_{k \ell}(x, y), \\
& \hat{F}_{k \ell}(t)=\left(F(x, y, t), \xi_{k \ell}(x, y)\right),
\end{aligned}
$$

where

$$
\begin{aligned}
& \xi_{k \ell}=e^{i(k x+\ell y)}, \\
& (u, v)=\frac{1}{L_{x} L_{y}} \int_{0}^{L_{x}} \int_{0}^{L_{y}} u v^{*} d x d y, \\
& k=\frac{2 \pi m}{L_{x}} \quad m=0, \pm 1 \ldots \pm M, \quad \ell=\frac{2 \pi n}{L_{y}} \quad n=0, \pm 1 \ldots \pm N,
\end{aligned}
$$




$$
\left(\xi_{k \ell}, \xi_{k^{\prime} \ell^{\prime}}\right)=\left\{\begin{array}{l}
0 \text { if } k \neq k^{\prime} \text { or } \ell \neq \ell^{\prime} \\
1 \text { if } k=k^{\prime} \text { and } \ell=\ell^{\prime}
\end{array}\right. \text {, }
$$

and $F(x, y, t)$ represents any of the dependent variables.

When series expansions of the form of (3.7) are substituted into the governing equations and the inner product is taken with each basis function $\xi_{k \ell}$, the constant coefficient linear terms are transformed in a very simple way. This can be seen by considering a term of the form $\frac{\partial F}{\partial x}$. Applying the Galerkin procedure to this term gives

$$
\begin{aligned}
\frac{\partial F}{\partial x} \rightarrow & \left(\frac{\partial}{\partial x} \sum_{k \ell} \hat{F}_{k \ell}(t) \xi_{k \ell^{\prime}}, \xi_{k^{\prime} \ell^{\prime}}\right) \\
& +\sum \sum i k \hat{F}_{k \ell}(t)\left(\xi_{k \ell}, \xi_{k^{\prime} \ell^{\prime}}\right) \\
& \quad k \ell \\
\frac{\partial F}{\partial x} \rightarrow & i k \hat{F}_{k \ell}(t)
\end{aligned}
$$

where the orthonormality condition (3.12) has been used. Similarly, other constant coefficient linear terms will be transformed as follows:

$$
\begin{aligned}
& \frac{\partial F}{\partial y} \rightarrow i \ell \hat{F}_{k \ell}(t) \\
& \frac{\partial^{2} F}{\partial x^{2}} \rightarrow-k^{2} \hat{F}_{k \ell}(t) \\
& \frac{\partial^{2} F}{\partial y^{2}} \rightarrow-\ell^{2} \hat{F}_{k \ell}(t) \\
& \frac{\partial F}{\partial t} \rightarrow \frac{d \hat{F}_{k \ell}(t)}{d t} .
\end{aligned}
$$


The transformation of the nonlinear terms is more complicated and will be discussed in the next section. However, nonlinear terms of the form $\frac{\partial}{\partial x}(F G)$ can be integrated by parts as follows:

$$
\begin{aligned}
\frac{\partial}{\partial x}(F G) & \rightarrow\left(\frac{\partial}{\partial x}[F G], \xi_{k^{\prime} \ell^{\prime}}\right) \\
& \rightarrow\left([F G],-\frac{\partial}{\partial x}\left[\xi_{k^{\prime} \ell^{\prime}}\right]\right) \\
\frac{\partial}{\partial x}(F G) & \rightarrow i k\left(F G, \xi_{k^{\prime} \ell^{\prime}}\right) .
\end{aligned}
$$

Similarly,

$$
\frac{\partial}{\partial y}(F G) \rightarrow i \ell\left(F G, \xi_{k^{\prime} \ell^{\prime}}\right)
$$

Substituting truncated series expansions of the form of (3.7) into (2.38)-(2.42) and (2.47), taking the inner product with each basis function and using (3.13) then gives

$$
\begin{aligned}
& \frac{d \hat{\zeta}_{i k \ell}}{d t}+f_{0} \hat{\delta}_{i k \ell}+i k \beta \hat{\psi}_{i k \ell}= \\
& \quad-i k \hat{A}_{i k \ell}-i \ell \hat{B}_{i k \ell}+i k\left(\hat{V}_{y i k \ell}+\hat{F}_{y i k \ell}\right)-i \ell\left(\hat{V}_{x i k \ell}+\hat{F}_{x i k \ell}\right), \\
& \frac{d \hat{\delta}_{i k \ell}}{d t}-f_{o} \hat{\zeta}_{i k \ell}+i k \beta \hat{x}_{i k \ell}-\left(k^{2}+\ell^{2}\right) \hat{\phi}_{i k \ell}= \\
& -i \ell \hat{A}_{i k \ell}+i k \hat{B}_{i k \ell}+i \ell\left(\hat{v}_{y i k \ell}+\hat{F}_{y i k \ell}\right)+i k\left(\hat{V}_{x i k \ell}+\hat{F}_{x i k \ell}\right)+\left(k^{2}+\ell^{2}\right) \hat{E}_{i k \ell} \\
& H_{0} \hat{\delta}_{o k \ell}+\hat{w}_{k \ell}=0
\end{aligned}
$$




$$
\begin{aligned}
& \frac{d \hat{h}_{1 k \ell}}{d t}+H_{1} \hat{\delta}_{1 k \ell}-\hat{w}_{k \ell}=-i k \hat{C}_{1 k \ell}-i \ell \hat{D}_{1 k \ell}-\hat{Q}_{k \ell} \\
& \frac{d \hat{h}_{2 k \ell}}{d t}+H_{2} \hat{\delta}_{2 k \ell}=-i k \hat{C}_{2 k \ell}-i \ell \hat{D}_{2 k \ell}+\frac{1}{\varepsilon} \hat{Q}_{k \ell} \\
& \frac{d \hat{\Lambda}_{0}}{d t}=-\hat{J}_{o k \ell}-\hat{K}_{o k \ell}-\hat{L}_{o k \ell}+\hat{M}_{o k \ell}-\lambda\left(k^{2}+\ell^{2}\right) \hat{\Lambda}_{o k \ell}
\end{aligned}
$$

where

$$
\begin{array}{ll}
A_{i}=u_{i} \zeta_{i} & B_{i}=v_{i} \zeta_{i} \\
C_{i}=u_{i} h_{i} & D_{i}=v_{i} h_{i} \\
E_{i}=\left(\frac{u_{i}^{2}+v_{i}^{2}}{2}\right) & K_{0}=v_{0} \frac{\partial \Lambda_{0}}{\partial y} \\
J_{0}=u_{0} \frac{\partial \Lambda_{0}}{\partial x} & \\
L_{0}=\frac{w^{-}}{H_{0}}\left(\Lambda_{0}-\Lambda_{1}\right) & \\
M_{0}=\frac{C_{E}}{H_{0}}\left|V_{0}\right|\left(\Lambda_{s}-\Lambda_{0}\right) .
\end{array}
$$

Equations (3.15)-(3.20) are the prognostic equations for the Fourier series amplitudes of the dependent variables which must be solved for all wavenumbers $k$ and $\ell$. If the above system of equations is linearized, all the terms on the right are set to zero, and the resulting set of equations can be solved separately for each wave number, since the linear terms are decoupled. This cannot be done in the nonlinear case, however, since the nonlinear terms contain products 
of the series expansions which couple all of the Fourier series amplitudes.

The diagnostic relations between the dependent variables can also be transformed to give diagnostic relations between the Fourier series amplitudes. As will be seen in the next section, the nonlinear products of the dependent variables which appear will be calculated in physical space so that it is only necessary to transform the linear diagnostic relations (2.49)-(2.52). Applying the Galerkin procedure to these equations gives

$$
\begin{array}{ll}
\hat{\zeta}_{i k \ell}=-\left(k^{2}+l^{2}\right) \hat{\psi}_{i k \ell} & \hat{\delta}_{i k \ell}=-\left(k^{2}+l^{2}\right) \hat{x}_{i k \ell} \\
\hat{u}_{i k \ell}=-i \ell \hat{\psi}_{i k \ell}+i k \hat{x}_{i k l} & \hat{v}_{i k \ell}=i k \hat{\psi}_{i k \ell}+i \ell \hat{x}_{i k \ell} \\
\hat{\phi}_{1 k \ell}=\hat{\phi}_{1 k \ell}=g\left(\hat{h}_{1 k \ell}+\varepsilon \hat{h}_{2 k \ell}\right) & \\
\hat{\phi}_{2 k \ell}=g\left(\hat{h}_{1 k \ell}+\hat{h}_{2 k \ell}\right) &
\end{array}
$$

\subsection{Evaluation of the Nonlinear Terms} Using the Transform Method

In order to solve (3.15)-(3.20) using time differencing, it is necessary to evaluate the nonlinear terms on the right side of each equation, given the series amplitudes of the dependent variables. As discussed in chapter 1 , the nonlinear terms can be calculated efficiently using the transform method introduced by Orszag (1970) and Eliasen et al. (1970). In the transform method, the dependent variables are first transformed from spectral to physical space on some specified grid. The nonlinear products are computed at the physical space grid points and then transformed to spectral space using an appropriate 
quadrature rule. When the basis functions are Fourier components, the transform from physical to spectral space can be calculated exactly for quadratic nonlinear terms provided $3 M+1$ or $3 N+1$ point trapezodial quadrature is used to evaluate the $x$ and $y$ integrals which appear.

To illustrate the transform method, consider the calculation of the term $\hat{A}_{1 k \ell}$ which represents the Fourier series amplitudes of the nonlinear product of $u_{1} \zeta_{1}$. Using $(3.8)-(3.10)$ and the definition of $A_{1}$ in (3.21) gives

$$
\hat{A}_{1 k l}=\frac{1}{L_{x} L_{y}} \int_{0}^{L_{y}} \int_{0}^{L_{x}} u_{1}(x, y, t) \zeta_{1}(x, y, t) e^{-i(k x+l y)} d x d y .
$$

Evaluating the $x$ and $y$ integrals in the above equations using $3 M+1$ and $3 N+1$ point trapezodial quadrature gives

$$
\hat{A}_{1 k \ell}=\frac{1}{(3 N+1)} \frac{1}{(3 M+1)} \sum_{q=0}^{3 N} \sum_{p=0}^{3 M} u_{1}\left(x_{p}, y_{q}, t\right) \zeta_{1}\left(x_{p}, y_{q}, t\right) e^{-i\left(k x_{p}+l y_{q}\right)}
$$

where

$$
\left.\begin{array}{l}
x_{p}=\left(\frac{L_{x}}{(3 M+1)}\right) p \\
y_{q}=\left(\frac{L_{y}}{(3 N+1)}\right) q
\end{array}\right\}
$$

and $N$ and $M$ are the truncation limits of the Fourier series. At any given time, the series amplitudes of the dependent variables are known from initial conditions or were calculated at the previous time step, so $u_{1}$ and $\zeta_{1}$ at the grid points $x_{p}, y_{q}$ can be calculated using (3.7) which gives 


$$
\left.\begin{array}{rl}
u_{1}\left(x_{p}, y_{q}, t\right)= & \sum \sum \hat{u}_{1 k \ell}(t) e^{i\left(k x_{p}+l y_{q}\right)} \\
& k \ell
\end{array}\right\}
$$

In summary, the nonlinear terms are evaluated by first transforming the needed dependent variables from spectral space to physical space at the grid points $x_{p}, y_{q}$ using equations of the form of (3.29). The nonl inear products are computed at the grid points and then transformed to spectral space using an equation of the form of (3.27).

In (3.26), $u_{1}(x, y, t)$ and $\zeta_{1}(x, y, t)$ are represented by truncated Fourier series with maximum wavenumbers of $\left(2 \pi / L_{x}\right) M$ and $\left(2 \pi / L_{y}\right) N$ in the $x$ and $y$ directions. The product of $u_{1}, \zeta_{1}$ and the exponential factor in the integrand of (3.26) is then represented by truncated Fourier series with maximum wave numbers of $\left(2 \pi / L_{x}\right) 3 M$ and $\left(2 \pi / L_{y}\right) 3 N$. From the theory of numerical quadrature (Krylov, 1962) it can be shown that $3 M+1$ and $3 \mathrm{~N}+1$ point trapezodial quadratures are exact if the integrand is a truncated Fourier series with a maximum wave number less than or equal to $\left(2 \pi / L_{x}\right) 3 M$ or $\left(2 \pi / L_{y}\right) 3 N$. Thus, quadratic nonlinear terms are evaluated exactly when (3.27) is used for the transformation from physical space to spectral space. Since the quadratic nonlinear terms are evaluated exactly, no aliasing error is introduced, which prevents nonlinear instability of the type described by Phillips (1959).

Some of the nonlinear terms which appear in the governing equations, such as the diabatic term $Q$ and the surface flux terms, are not quadratic in the dependent variables. For these terms, the trapezodial quadrature will not be exact, and some aliasing error will 
occur. Bourke et a1. (1977) have shown that in a 5-level hemispheric spectral model with moist physics, the aliasing error introduced by the higher order nonlinear terms is negligible. Thus, all the nonlinear terms are calculated using the minimum number of grid points necessary to resolve the quadratic nonlinear terms.

In a typical model run, most of the computing time is used to evaluate the series shown in (3.27) and (3.29). Both of these series can be written in terms of discrete Fourier transforms as shown in Appendix A, so that efficient Fast Fourier Transform (FFT) algorithms can be used. The current model uses FFT routines which were written in vector code for a Cray-1 computer. Fig. 3 shows the ratio of the computing time needed to transform a variable from spectral to physical and back to spectral space when the series are evaluated directly, to the time needed when the FFT algorithms are used, for various series truncations. In Fig. 3 it is assumed that the series truncation is the same in the $x$ and $y$ directions $(M=N)$. For the case when $M=N=35,(36$ Fourier modes in Fig. 3) which was used in most of the model simulations, the transforms can be evaluated about 13 times faster when the FFT algorithms are used. Since most of the model computing time is used for the transforms, the use of the FFT algorithm greatly increases the efficiency of the model.

\subsection{Derivation of the Model Normal Modes}

The linear terms which appear on the left side of $(3.15)-(3.20)$ are a system of nine coupled equations in nine unknowns, for each wavenumber $k$ and $\ell$. The linear terms can be simplified by transforming the 


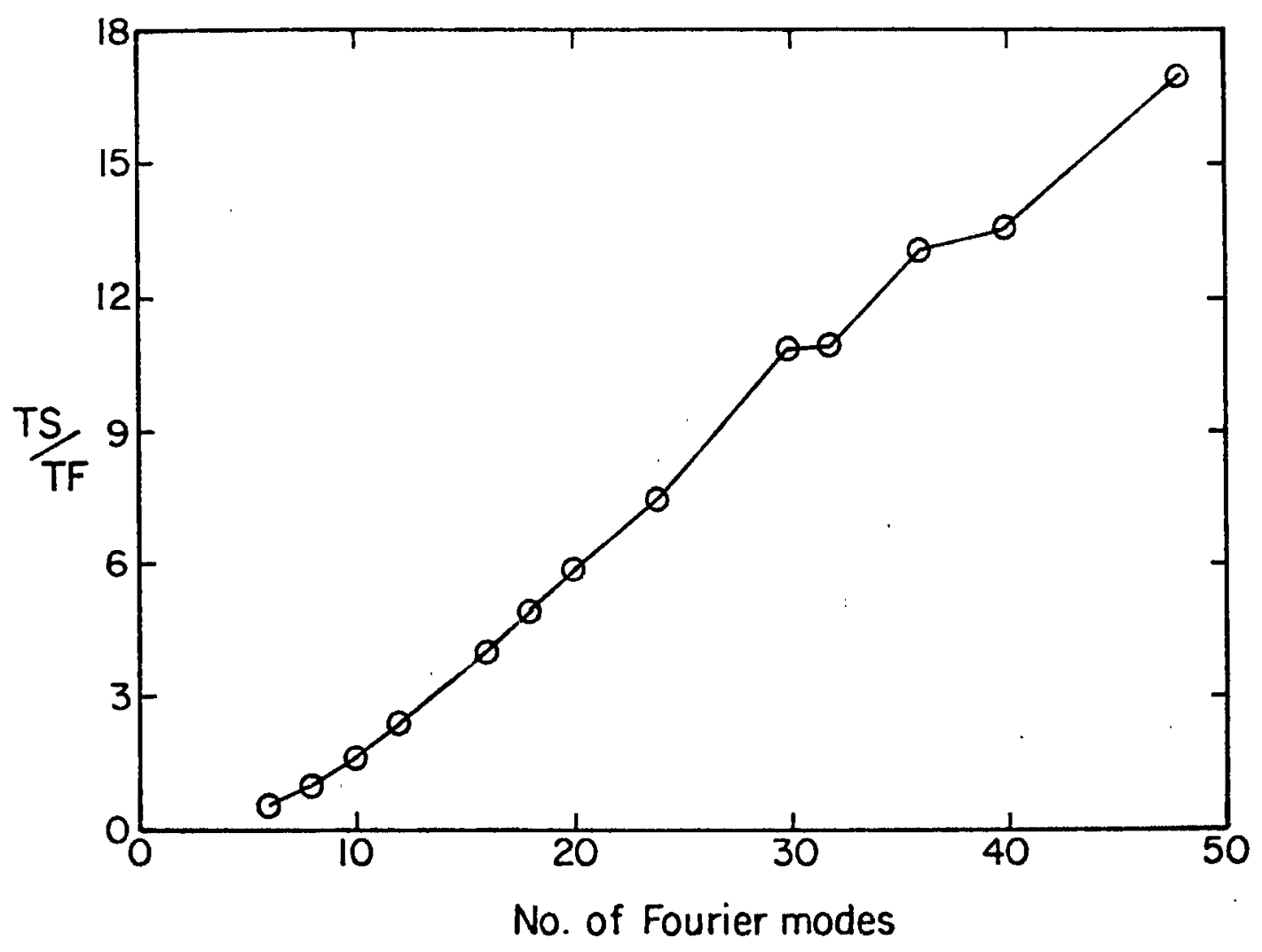

Figure 3. The ratio of computing time needed for two-dimensional transforms from spectral to physical and back to spectral space without FFTs to the time needed with FFTs. 
spectral equations into a system for the normal modes of the linear equations.

To simpify the discussion, it is convenient to eliminate $\hat{w}_{k \ell}$ between (3.17) and (3.18) and to use the diagnostic relations (3.22)(3.25) to write $(3.15)-(3.19)$ as

$$
\begin{aligned}
& -\left(k^{2}+\ell^{2}\right) \frac{d \hat{\psi}_{o k \ell}}{d t}-\left(k^{2}+\ell^{2}\right) f_{0} \hat{x}_{o k \ell}+i k \beta \hat{\psi}_{o k \ell} \quad=\hat{p}_{1 k \ell} \\
& -\left(k^{2}+\ell^{2}\right) \frac{d \hat{x}_{o k \ell}}{d t}+\left(k^{2}+\ell^{2}\right) f_{0} \hat{\psi}_{o k \ell}+i k B \hat{x}_{o k \ell}-g\left(k^{2}+\ell^{2}\right)\left(\hat{h}_{1 k \ell}+\varepsilon \hat{h}_{2 k \ell}\right)=\hat{p}_{2 k \ell} \\
& \frac{d \hat{h}_{1 k \ell}}{d t}-\left(k^{2}+\ell^{2}\right)\left(H_{1} \hat{x}_{1 k \ell}+H_{0} \hat{x}_{o k \ell}\right) \\
& -\left(k^{2}+\ell^{2}\right) \frac{d \hat{\psi}_{1 k \ell}}{d t}-\left(k^{2}+\ell^{2}\right) f_{0} \hat{x}_{1 k \ell}+i k \beta \hat{\psi}_{1 k \ell} \quad=\hat{p}_{4 k \ell} \\
& -\left(k^{2}+\ell^{2}\right) \frac{d \hat{x}_{1 k \ell}}{d t}+\left(k^{2}+\ell^{2}\right) f_{0} \hat{\psi}_{1 k \ell}+i k \beta \hat{x}_{1 k \ell}-g\left(k^{2}+\ell^{2}\right)\left(\hat{h}_{1 k \ell}+\varepsilon \hat{h}_{2 k \ell}\right)=\hat{p}_{5 k \ell} \\
& \frac{d \hat{h}_{2 k \ell}}{d t}-\left(k^{2}+\ell^{2}\right) H_{2} \hat{x}_{2 k \ell} \\
& -\left(k^{2}+l^{2}\right) \frac{d \hat{\psi}_{2 k \ell}}{d t}-\left(k^{2}+\ell^{2}\right) \hat{f}_{0} \hat{x}_{2 k \ell}+i k \beta \hat{\psi}_{2 k \ell} \\
& -\left(k^{2}+\ell^{2}\right) \frac{d \hat{x}_{2 k \ell}}{d t}+\left(k^{2}+\ell^{2}\right) f_{0} \hat{\psi}_{2 k \ell}+i k \beta \hat{x}_{2 k \ell}-g\left(k^{2}+\ell^{2}\right)\left(\hat{h}_{1 k \ell}+\hat{h}_{2 k \ell}\right)=\hat{p}_{8 k \ell} \\
& =\hat{p}_{3 k \ell} \\
& =\hat{\mathrm{P}}_{6 \mathrm{k} \ell} \\
& =\hat{\mathrm{P}}_{7 \mathrm{k} \ell}
\end{aligned}
$$

where $\hat{\mathrm{P}}_{1 \mathrm{k} \ell}$ through $\hat{\mathrm{P}}_{8 \mathrm{k} \ell}$ represent the nonlinear terms. The first step in finding the linear normal modes of the above equations is to decouple the dependent variables for each layer. This can be accomplished by using a procedure described by Veronis and Stomel (1956) for an oceanographic model. The basic idea of the method is to add and 
subtract the equations for each layer in such a way that the linear combinations of the dependent variables which appear are proportional to each other.

In order to decouple the layer 0 terms, (3.33) and (3.34) are subtracted from (3.30) and (3.31) which gives

$$
\begin{aligned}
& -\left(k^{2}+\ell^{2}\right) \frac{d \hat{S}_{o k \ell}}{d t}-\left(k^{2}+\ell^{2}\right) f_{0} \hat{V}_{o k \ell}+i k \beta \hat{S}_{o k \ell}=\hat{P}_{1 k \ell}-\hat{P}_{4 k \ell} \\
& -\left(k^{2}+\ell^{2}\right) \frac{d \hat{V}_{o k \ell}}{d t}+\left(k^{2}+\ell^{2}\right) f_{0} \hat{S}_{o k \ell}+i k \beta \hat{V}_{o k \ell}=\hat{P}_{2 k \ell}-\hat{P}_{5 k \ell}
\end{aligned}
$$

where

$$
\left.\begin{array}{l}
\hat{s}_{o k l}=\hat{\psi}_{o k \ell}-\hat{\psi}_{1 k \ell} \\
\hat{v}_{o k l}=\hat{x}_{o k \ell}-\hat{x}_{1 k \ell}
\end{array}\right\}
$$

Adding $\frac{H_{1}}{H_{0}}$ times (3.33) and (3.34) to (3.30) and (3.31), and rewriting (3.32) gives

$$
\begin{aligned}
& \frac{d \hat{h}_{1 k \ell}}{d t}-\left(k^{2}+\ell^{2}\right) H_{0} \hat{x}_{s k \ell}=\hat{p}_{3 k \ell} \\
&-\left(k^{2}+\ell^{2}\right) \frac{d \hat{\psi}_{s k \ell}}{d t}-\left(k^{2}+\ell^{2}\right) f_{0} \hat{x}_{s k \ell}+i k \beta \hat{\psi}_{s k \ell}=\hat{p}_{1 k \ell}+\frac{H_{1}}{H_{0}} \hat{p}_{4 k \ell} \\
&-\left(k^{2}+l^{2}\right) \frac{d \hat{x}_{s k \ell}}{d t}+\left(k^{2}+\ell^{2}\right) f_{0} \hat{\psi}_{s k \ell}+i k \beta \hat{x}_{s k \ell}-g\left(1+\frac{H_{1}}{H_{0}}\right)\left(k^{2}+\ell^{2}\right)\left(\hat{h}_{1 k \ell}+\varepsilon \hat{h}_{2 k \ell}\right) \\
&=\hat{p}_{2 k \ell}+\frac{H_{1}}{H_{0}} \hat{p}_{5 k \ell}
\end{aligned}
$$

where 


$$
\left.\begin{array}{l}
\hat{\psi}_{\text {skl }}=\hat{\psi}_{\text {okl }}+\frac{H_{1}}{H_{0}} \hat{\psi}_{1 k \ell} \\
\hat{x}_{\text {skl }}=\hat{x}_{\text {okl }}+\frac{H_{1}}{H_{0}} \hat{x}_{1 k \ell}
\end{array}\right\} .
$$

Equations (3.38)-(3.39) and (3.41)-(3.43) can be used in place of $(3.30)-(3.34)$. When this is done, the equations for $\hat{S}_{\text {oke }}$ and $\hat{V}_{\text {oke }}$ are decoupled from the remaining six equations in the linear case.

In order to decouple the variables with subscripts $s$ or 1 from the layer 2 variables, $(3.41)-(3.43)$ are added to arbitrary constants $c_{1}, c_{2}$ and $c_{3}$ times (3.35)-(3.37). When this is done, several combinations of the dependent variables appear in the resulting three equations. Requiring the combinations of dependent variables which appear to be proportional gives the following relations

$$
\left.\begin{array}{c}
\left(\hat{\psi}_{s k l}+c_{3} \hat{\psi}_{2 k \ell}\right)=d_{1}\left(\hat{\psi}_{s k \ell}+c_{2} \hat{\psi}_{2 k \ell}\right) \\
\left(\hat{x}_{s k l}+c_{2} \hat{x}_{2 k \ell}\right)=d_{2}\left(\hat{x}_{s k \ell}+c_{3} \hat{x}_{2 k \ell}\right) \\
\left(H_{0} \hat{x}_{s k \ell}+c_{1} H_{2} \hat{x}_{2 k \ell}\right)=d_{3}\left(\hat{x}_{s k \ell}+c_{3} \hat{x}_{2 k \ell}\right) \\
\left\{\left[\left(1+\frac{H_{1}}{H_{0}}\right)+c_{3}\right] \hat{h}_{1 k \ell}+\left[\varepsilon\left(1+\frac{H_{1}}{H_{0}}\right)+c_{3}\right] \hat{h}_{2 k \ell}\right\}=d_{4}\left(\hat{h}_{1 k \ell}+c_{1} \hat{h}_{2 k \ell}\right)
\end{array}\right\},
$$

where $d_{1}$ through $d_{4}$ are constants of proportionality. In order for the above equations to be satisfied, the following conditions must be met:

$$
\begin{aligned}
& d_{1}=d_{2}=1, \quad d_{3}=H_{0}, \quad d_{4}=1+\frac{H_{1}}{H_{0}}+\frac{H_{2}}{H_{0}} c_{1}, \\
& c_{3}=c_{2}=\frac{H_{2}}{H_{0}} c_{1},
\end{aligned}
$$


and $c_{1}$ has two solutions which are the roots of

$$
c_{1}^{2}+c_{1}\left(\frac{H_{0}+H_{1}}{H_{2}}-1\right)-\varepsilon\left(\frac{H_{0}+H_{1}}{H_{2}}\right)=0 .
$$

Using (3.45)-(3.48), the sum of (3.41)-(3.43) with $c_{1}, c_{2}$ or $c_{3}$ times (3.35) - (3.37) can then be written as

$$
\begin{gathered}
\frac{d \hat{G}_{j k \ell}}{d t}-\left(k^{2}+l^{2}\right) H_{0} \hat{V}_{j k \ell}=\hat{P}_{3 k \ell}+c_{j} \hat{P}_{6 k \ell} \\
-\left(k^{2}+l^{2}\right) \frac{d \hat{S}_{j k \ell}}{d t}-\left(k^{2}+l^{2}\right) f_{0} \hat{V}_{j k \ell}+i \beta k \hat{S}_{j k \ell}=\hat{P}_{1 k \ell}+\frac{H_{1}}{H_{0}} \hat{P}_{4 k \ell}+\frac{H_{2}}{H_{0}} c_{j} \hat{P}_{7 k \ell} \\
-\left(k^{2}+l^{2}\right) \frac{d \hat{V}_{j k \ell}}{d t}+\left(k^{2}+l^{2}\right) f_{0} \hat{S}_{j k \ell}+i \beta k \hat{V}_{j k l}-g_{j}\left(k^{2}+l^{2}\right) \hat{G}_{j k \ell} \\
=\hat{P}_{2 k \ell}+\frac{H_{1}}{H_{0}} \hat{P}_{5 k \ell}+\frac{H_{2}}{H_{0}} c_{j} \hat{P}_{8 k \ell}
\end{gathered}
$$

where

$$
\left.\begin{array}{l}
\hat{G}_{j k l}=\hat{h}_{1 k \ell}+c_{j} \hat{h}_{2 k l} \\
\hat{S}_{j k l}=\hat{\psi}_{o k l}+\frac{H_{1}}{H_{0}} \hat{\psi}_{1 k l}+\frac{H_{2}}{H_{0}} c_{j} \hat{\psi}_{2 k l} \\
\hat{v}_{j k l}=\hat{x}_{o k \ell}+\frac{H_{1}}{H_{0}} \hat{x}_{1 k l}+\frac{H_{2}}{H_{0}} c_{j} \hat{x}_{2 k l}
\end{array}\right\}
$$

and $c_{j}, j=1,2$ are the roots of 


$$
c^{2}+c\left(\frac{H_{0}+H_{1}}{H_{2}}-1\right)-\varepsilon\left(\frac{H_{0}+H_{1}}{H_{2}}\right)=0 .
$$

Equations (3.49)-(3.51) for $j=1,2$ and (3.38)-(3.39) for $j=0$ can be used in place of (3.30)-(3.37). The new dependent variables $G_{j k \ell}, S_{j k \ell}$ and $V_{j k \ell}$ are now decoupled in the linear case for $j=0,1$ or 2 .

The linear terms can be simplified further by transforming the governing equations so that the remaining combinations of linear terms which appear are decoupled. For convenience, (3.49)-(3.51) and (3.38)(3.39) can be written in vector form as

$$
\frac{d \tilde{W}_{j k \ell}}{d t}+\tilde{A}_{j k \ell} \tilde{W}_{j k \ell}=\tilde{P}_{j k \ell} \quad j=0,1,2
$$

where

$$
\begin{aligned}
& \tilde{W}_{j k \ell}=\left(\begin{array}{c}
\hat{G}_{j k \ell} \\
\hat{S}_{j k \ell} \\
\hat{V}_{j k \ell}
\end{array}\right) \quad j=1,2 \quad \hat{W}_{o k \ell}=\left(\begin{array}{c}
\hat{S}_{o k \ell} \\
\hat{V}_{o k \ell}
\end{array}\right) \\
& \tilde{P}_{j k \ell}=\left(\begin{array}{c}
{\left[\hat{P}_{3 k \ell}+c_{j} \hat{P}_{6 k l}\right]} \\
-\left(\frac{1}{k^{2}+l^{2}}\right)\left[\hat{P}_{1 k \ell}+\frac{H_{1}}{H_{0}} \hat{P}_{4 k \ell}+\frac{H_{2}}{H_{0}} c_{j} \hat{P}_{7 k \ell}\right] \\
-\left(\frac{1}{k^{2}+l^{2}}\right)\left[\hat{P}_{2 k \ell}+\frac{H_{1}}{H_{0}} \hat{P}_{5 k \ell}+\frac{H_{2}}{H_{0}} c_{j} \hat{P}_{8 k \ell}\right]
\end{array}\right) \quad j=1,2 \\
& \tilde{\mathrm{P}}_{\text {okl }}=\left(\begin{array}{l}
-\left(\frac{1}{\mathrm{k}^{2}+\ell^{2}}\right)\left[\hat{\mathrm{P}}_{1 k \ell}-\hat{\mathrm{P}}_{4 k \ell}\right] \\
-\left(\frac{1}{\mathrm{k}^{2}+\ell^{2}}\right)\left[\hat{\mathrm{P}}_{2 k \ell}-\hat{\mathrm{P}}_{5 \mathrm{k} \ell}\right]
\end{array}\right)
\end{aligned}
$$




$$
\begin{aligned}
& \tilde{A}_{j k \ell}=\left(\begin{array}{ccc}
0 & 0 & -H_{0}\left(k^{2}+l^{2}\right) \\
0 & \frac{-i \beta k}{\left(k^{2}+l^{2}\right)} & f_{0} \\
g_{j} & -f_{0} & \frac{-i \beta k}{\left(k^{2}+l^{2}\right)}
\end{array}\right) j=1,2 \\
& \tilde{A}_{o k \ell}=\left(\begin{array}{cc}
\frac{-i \beta k}{\left(k^{2}+l^{2}\right)} & f_{0} \\
-f_{0} & \frac{-i \beta k}{\left(k^{2}+l^{2}\right)}
\end{array}\right) .
\end{aligned}
$$

For $j=1,2$, the terms on the left side of equation (3.55) represent a linear system of three coupled equations in three unknowns. These equations can be transformed into three scalar equations where the linear terms are decoupled, as shown by Cane and Sarachik (1976) for an equatorial $\beta-p l a n e$ model. To do this, let the inner product of two column vectors $\tilde{u}_{j k \ell}$ and $\tilde{v}_{j k \ell}$ with the dimensions of $\tilde{W}_{j k \ell}$ be defined by

$$
\left(\tilde{u}_{j k \ell}, \tilde{v}_{j k \ell}\right)=\left(d_{j k \ell}^{2} u_{1} v_{1}^{*}+u_{2} v_{2}^{*}+u_{3} v_{3}^{*}\right)
$$

where $u_{1}, v_{1}$ etc. are the components of $u_{j k \ell}$ and $v_{j k \ell},()^{*}$ denotes complex conjugate and $d_{j k \ell}^{2}$ will be determined later to make the inner product dimensionally correct. Using the above inner product, a scalar transform can be defined as follows

$$
W_{j k \ell r}=\frac{1}{E_{j k \ell r}}\left(\tilde{W}_{j k \ell}, \tilde{K}_{j k \ell r}\right)
$$

where $\tilde{K}_{j k \ell r}$ is a three component vector which is the kernel of the transform and $E_{j k e r}$ is a normalization factor. When (3.55) is transformed using (3.60), the linear terms will decouple provided that the kernel of the transform has the following property: 


$$
\tilde{A}_{j k \ell} \tilde{K}_{j k \ell r}+i v_{j k \ell r} \tilde{K}_{j k \ell r}=0 \text {. }
$$

The above equation implies that $\tilde{K}_{j k \ell r}$ are the eigenvectors of the matrix $\tilde{A}_{j k \ell}$ with corresponding eigenvalues -iv $j k \ell \cdot$. If $\tilde{A}_{j k \ell}$ has the property

$$
\left(\tilde{A}_{j k \ell} \tilde{u}_{j k \ell}, \tilde{v}_{j k \ell}\right)=-\left(\tilde{u}_{j k \ell}, \tilde{A}_{j k \ell} \tilde{v}_{j k \ell}\right) \text {, }
$$

then $\tilde{A}_{j k \ell}$ is skew-Hermitian with respect to the inner product (3.59) and the eigenvectors $\tilde{K}_{j k \ell}$ will be orthogonal. Appendix $B$ shows that (3.62) is satisfied provided that $\mathrm{d}_{j k \ell}^{2}$ is given by

$$
d_{j k \ell}^{2}=\frac{g_{j}}{H_{0}\left(k^{2}+\ell^{2}\right)} .
$$

and that $\tilde{K}_{j k \ell r}$ is given by

$$
\tilde{K}_{j k \ell r}=\left(\begin{array}{c}
f_{0} \\
\frac{\beta k v_{j k \ell r}-v_{j k \ell r}^{2}\left(k^{2}+l^{2}\right)}{\left(k^{2}+l^{2}\right)^{2} H_{0}}+g_{j} \\
\frac{i v_{j k \ell r} f_{0}}{H_{0}\left(k^{2}+l^{2}\right)}
\end{array}\right) j=1,2
$$

and $\nu_{j k \ell}$ for $r=1,2,3$ are the three roots of

$$
\nu_{j k \ell r}\left(\bar{v}_{j k \ell r}^{2}-f_{0}^{2}\right)=g_{j} H_{0}\left(k^{2}+l^{2}\right) \bar{v}_{j k \ell r}
$$

where $\bar{v}_{j k \ell r}=v_{j k \ell r}-\frac{\beta k}{k^{2}+\ell^{2}}$. For the case when $j=0$, the scalar transform in (3.60) is defined by the inner product (3.59) except that the first term on the right does not appear. For this case, the kernel is given by 


$$
\tilde{\mathrm{k}}_{\mathrm{ok \ell r}}=\left(\begin{array}{c} 
\pm i \\
1
\end{array}\right) \text {, }
$$

with

$$
v_{0 k \ell r}=\frac{\beta k}{\left(k^{2}+\ell^{2}\right)} \pm f_{0} \text {, }
$$

where either the plus or minus sign is chosen for $r=1$ or 2 .

Now, taking the inner product of $(3.55)$ with $\tilde{K}_{j k e r}$ and multiplying by $E_{j k \ell r}^{-1}$ gives

$$
\frac{d W_{j k \ell r}}{d t}+\frac{1}{E_{j k \ell r}}\left(\tilde{A}_{j k \ell} \tilde{W}_{j k \ell}, \tilde{K}_{j k \ell r}\right)=P_{j k \ell r},
$$

where $W_{j k \ell r}$ is defined by $(3.60)$ and $P_{j k \ell r}$ is given by

$$
P_{j k \ell r}=\frac{1}{E_{j k \ell r}}\left(\tilde{P}_{j k \ell}, \tilde{K}_{j k \ell r}\right) \text {. }
$$

Using (3.62) and (3.61), (3.68) becomes

$$
\frac{d w_{j k \ell r}}{d t}-i v_{j k \ell r} w_{j k \ell r}=P_{j k \ell r} .
$$

The above equation is the transformed version of (3.55) which must be solved for $j=0,1,2 ; r=1,2,3$ and for each wave number $k$ and $\ell$. Since $\tilde{A}_{j k \ell}$ is skew-Hermitian the eigenvectors $\tilde{K}_{j k \ell}$ are orthogonal, and the inverse of the scalar transform shown in (3.60) is given by

$$
\tilde{W}_{j k \ell}=\sum_{r} w_{j k \ell r} \tilde{K}_{j k \ell r} \begin{cases}r=1,2,3 & j=1,2 \\ r=1,2 & j=0\end{cases}
$$

provided that the normalization factor $E_{j k \ell r}$ is given by 


$$
E_{j k \ell r}=\left(\tilde{K}_{j k \ell r}, \tilde{K}_{j k \ell r}\right)
$$

The advantage to using (3.70) instead of (3.30)-(3.37) is that all of the linear terms have been reduced to the form of an oscillation equation. For the linear case, $P_{j k \ell r}=0$ and the solution to $(3.70)$ is given by

$$
w_{j k \ell r}(t)=w_{j k \ell r}(0) e^{i \nu_{j k \ell r} t}
$$

where $w_{j k \ell r}(0)$ is determined from initial conditions. Thus, $w_{j k \ell r}$ are the amplitudes of normal modes of the linear equations, which oscillate with the frequencies $\nu_{j k \ell}$.

For $j=1,2$ the frequencies $\nu_{j k e r}$ are given by (3.65). If it is assumed that $v_{j k \ell r}$ is approximately equal to $\bar{v}_{j k \ell r}$, then (3.65) becomes

$$
\nu_{j k \ell r}^{2}=g_{j} H_{0}\left(k^{2}+l^{2}\right)+f_{0}^{2} .
$$

In the above form, it can be seen that two of the frequencies for $j=1$ or 2 correspond to gravity-inertia waves. The quantity $\sqrt{g_{j} H_{0}}$ can then be interpreted as the phase speed of a pure gravity wave. Using the values of the parameters listed in Table $1, \sqrt{g_{j} H_{0}}$ takes the values of $324 \mathrm{~ms}^{-1}$ and $52 \mathrm{~ms}^{-1}$ for $j=1$ or 2 . These are similar to the pure gravity wave speeds for the external and the first internal vertical modes of a fully stratified model (Fulton and Schubert, 1980). If it is assumed that $\bar{v}_{j k \ell r}$ is much smaller than $f_{0}$, then (3.65) becomes

$$
\nu_{j k \ell r}=\frac{\beta k}{k^{2}+\ell^{2}+f_{0}^{2} / g_{j} H_{0}}
$$

From the above equation it can be seen that the third frequency for $j=1$ or 2 corresponds to a Rossby wave. 
When $j=0$, the frequencies are given by (3.67). For this case, the frequencies correspond to inertial oscillations which are slightiy modified by $\beta$. There are only two frequencies for this case because the height of the lowest layer was assumed to be constant. Because the dependent variables for $j=0$ are related to the difference between the layer 0 and layer 1 variables, the frequencies for this case will be referred to as the boundary layer modes.

Some of the simulations presented in later chapters were run on an $f-p l$ ane $(\beta=0)$ rather than the $\beta-p l$ ane. For this case, $(3.74)$ and $(3.75)$ are exact for $j=1$ or 2 . The gravity wave frequencies given by (3.74) are approximately the same as for the $\beta-p l a n e$ case, but the Rossby wave frequencies given by (3.75) are identically zero. For the f-plane case, these modes correspond to the part of the solution which is in exact geostrophic balance. In the linear case, these modes do not change with time. For convenience, the modes which do not correspond to gravity waves for $j=1$ or 2 will be referred to as rotational modes for the $f$ plane case and Rossby modes for the $\beta-p l a n e$ case.

In summary, (3.30)-(3.37) have been transformed to give (3.70) which must be solved for $j=0,1,2 ; r=1,2,3$ and for each wavenumber $k$ and $\ell$ where $W_{j k \ell r}$ are the amplitudes of the normal modes of the linear governing equations. The normal modes oscillate with the frequencies $v_{j k \ell r}$ which correspond to external or internal gravity or Rossby waves when $j=1$ or 2 and $r=1,2$ or 3 and to inertial oscillations when $j=0$. Fig. 4 shows the frequencies for each $j$ and $r$ as a continuous function of $k$ for several meridional wavenumbers $n\left(\ell=2 \pi n / L_{y}\right)$ for a 4000 by 4000 $\mathrm{km}$ domain. Fig. 4a shows the external and internal gravity wave frequencies and Fig. 4b shows the external and internal Rossby wave 

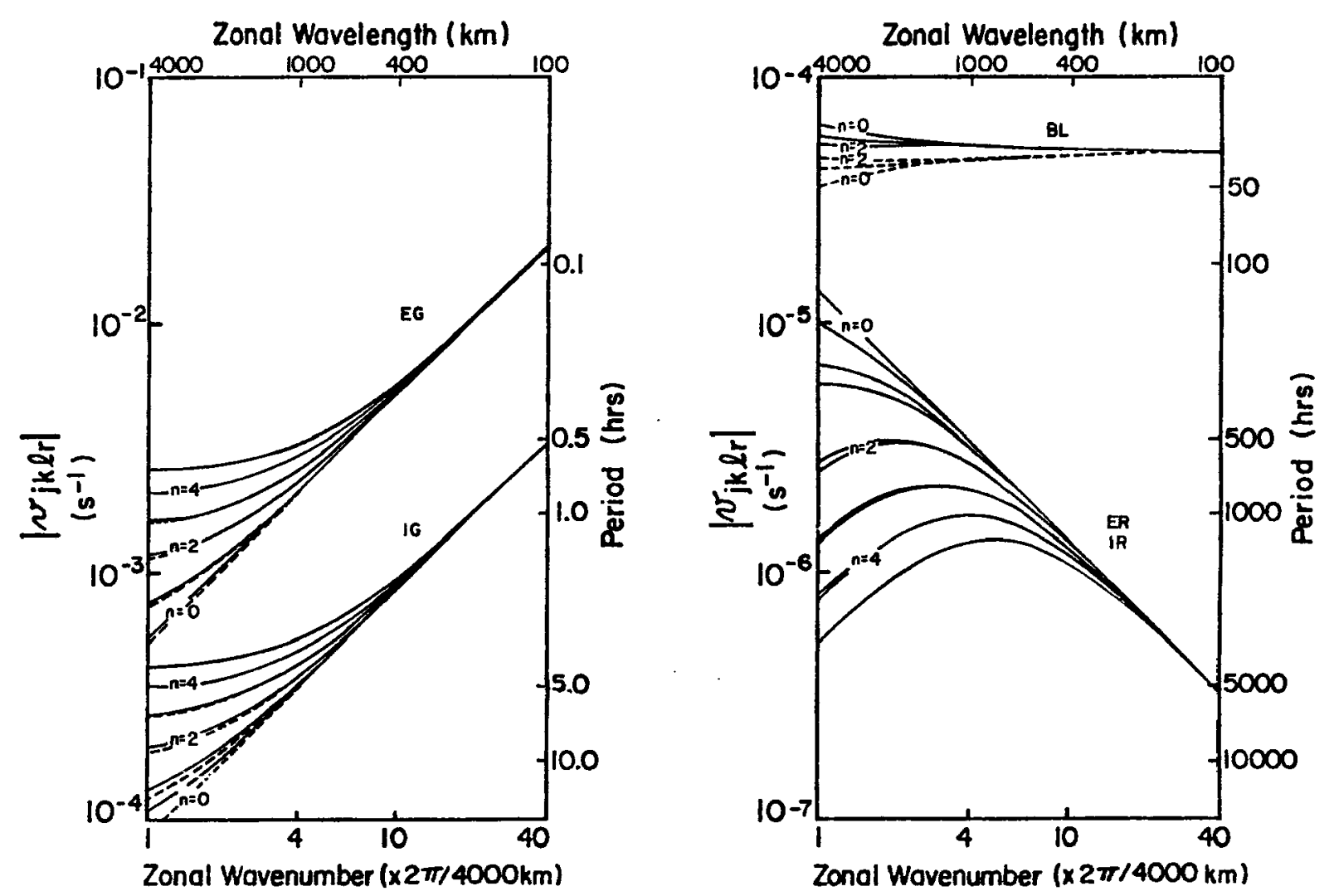

Figure 4. The frequencies of the normal modes of the linearized governing equations for a 4000 by $4000 \mathrm{~km}$ domain where the solid lines indicate positive frequencies (westward propogation) and the dashed 1 ines indicate negative frequencies (eastward propogation). Fig. (a) shows the external gravity waves (EG) and internal gravity waves (IG) and Fig. (b) shows the external and internal Rossby waves (ER) and (IR) and the modes associated with the constant depth boundary layer (BL). 
frequencies and the frequencies associated with the constant depth boundary layer. Comparing Fig. $4 a$ with Fig. $4 b$ it can be seen that there is a large frequency separation between the gravity and Rossby waves with the frequencies of the inertial oscillations about halfway between the two. The Rossby wave frequencies for the external and internal modes occur in pairs and asymptotically become equal for high wave numbers, while the frequencies of the external gravity waves are about a factor of six larger than the frequencies of the internal gravity waves for all wavenumbers.

\subsection{Time Discretization}

In the general case the transformed governing equations are nonlinear so they must be solved using some type of time differencing. If a fully explicit scheme is applied, the size of the time step which can be used will be limited by a stability condition of the form $\sigma \Delta t \leq 1$ where $\Delta t$ is the time step and $\sigma$ is the maximum frequency which is allowed in the model. In the current model, this will be the frequency of the shortest wavelength external gravity waves which have a phase speed on the order of $300 \mathrm{~ms}^{-1}$. Since the motion of the external gravity waves is governed by the linear terms of the model, a larger time step can be used if the linear terms are treated implicitly. When the governing equations are written in the form of (3.70), the linear terms can be treated implicitly with no more work than is required for an explicit scheme. A slight disadvantage of using a semi-implicit scheme, however, is that although the amplitude of the external gravity waves would not increase, some error in their phase would be introduced. In the current model, this problem can be avoided by multiplying (3.70) 
by an integrating factor $e^{-i \nu j k \ell r t}$ which gives

$$
\frac{d}{d t}\left(w_{j k \ell r} e^{-i \nu_{j k \ell r} t}\right)=P_{j k \ell r} e^{-i \nu_{j k \ell r} t} \text {. }
$$

If the quantity inside the time derivative is considered the new dependent variable, the above equation can be solved using time differencing where the stability criteria is related to the nonlinear terms, without distortion of the amplitude or the phase of the external gravity waves.

Letting $t=\tau \Delta t$ and using forward time differencing for the first time step, and the second order Adams-Bashforth scheme for all subsequent time steps gives

$$
\begin{aligned}
w_{j k \ell r}^{(1)}=\left(w_{j k \ell r}^{(0)}\right. & \left.+\Delta t P_{j k \ell r}^{(0)}\right) e^{i \nu_{j k \ell r} \Delta t} \\
w_{j k \ell r}^{(\tau+1)}=w_{j k \ell r}^{(\tau)} e^{i \nu_{j k \ell r} \Delta t} & \\
& +\Delta t\left[3 / 2 P_{j k \ell r}^{(\tau)} e^{i v_{j k \ell r} \Delta t}-\frac{1}{2} P_{j k \ell r}^{(\tau-1)} e^{\left.i \nu_{j k \ell r^{2 \Delta t}}\right]}\right.
\end{aligned}
$$

where the superscript indicates the time level. The Adams-Bashforth scheme was chosen over the leap-frog scheme because it can be applied to both the oscillation and decay equations. Since the leapfrog scheme is unstable for the decay equation, it would be necessary to treat the damping terms which appear in $P_{j k \ell r}$ by some other method. Another advantage to using the Adams-Bashforth scheme is that the computational mode which appears in any three-level scheme is damped for both the oscillation and the decay equation. A slight disadvantage of the AdamsBashforth scheme is that although the computational mode is damped, the 
physical mode is slightly unstable. This is not a major problem, however, since the amplification factor is proportional to $(\Delta t)^{4}$ so that for small time steps, the growth rate becomes negligible. For a typical model simulation there was no sign of instability by the end of a four day integration. If the weak instability of the second-order AdamsBashforth scheme did prove to be a problem, this difficulty could be removed as shown by Gazdag (1976). Using the Adams-Bashforth scheme as a predictor step and the trapezodial scheme as a corrector step removes the weak instability of the second-order Adams-Bashforth scheme.

When the governing equations in the form of (3.76) are solved using the time differencing scheme shown in (3.77)-(3.79), it is necessary to determine an appropriate time step. Since (3.76) is nonlinear, the stability properties are difficult to determine. From numerical experiments, a useful guide for determining the time step was found to be

$$
\Delta t \leq \frac{1}{2|W|\left(K^{2}+L^{2}\right)^{\frac{1}{2}}}
$$

where $|\mathbb{V}|$ is the maximum wind speed on the physical space grid and $K$ and $L$ are the maximum wavenumbers. For a $3600 \mathrm{~km}$ by $3600 \mathrm{~km}$ domain and wavenumber truncation limits $M=N=35$ and a maximum wind speed of $60 \mathrm{~ms}^{-1}$, $\Delta t$ is about 97 seconds. In most of the model simulations, a time step of 90 seconds was used. If the linear terms were treated explicitly, the stability criteria of the form $\sigma \Delta t \leq 1$ with $\sigma$ the maximum frequency of the external gravity waves would require a time step of about 36 seconds. Thus, the exact treatment of the linear terms increases the model efficiency by a factor of about 2.5 . 


\subsection{Nonlinear Normal Mode Initialization}

When the governing equations are written in the form of (3.70), the dependent variables represent the amplitudes of the normal modes of the linearized equations. When written in this form, the application of the nonlinear normal mode initialization procedure proposed by Machenhauer (1977) is straightforward.

The first step in applying the procedure is to divide $W_{j k 2 r}$ into a slow (low frequency) mode part and a fast (high frequency) mode part as follows

$$
W_{j k \ell r}=W_{j k \ell r}^{s}+W_{j k \ell r}^{f}
$$

As can be seen in Fig. 4, the Rossby waves can be considered the slow modes and the gravity waves can be considered the fast modes since there is suct a large frequency separation between these waves. The frequencies of the boundary layer modes, however, lie about halfway between the Rossby and gravity wave frequencies. In practice it was necessary to consider the boundary layer modes to be slow to obtain convergence of the initialization scheme.

Now, suppose the initial values of the dependent variables are given so that $W_{j k \ell r}^{S}$ and $W_{j k \ell r}^{f}$ can be computed. To apply Machenauer's initialization scheme, the slow mode contribution is retained and the fast mode contribution is discarded. The fast mode contribution is then recomputed assuming that $\frac{d W_{j k \ell r}^{f}}{d t}$ is small enough so that (3.70) gives

$$
W_{j k \ell r}^{f}=\frac{i}{v_{j k \ell r}^{f}} P_{j k \ell r}^{f}
$$

Since the nonlinear terms couple all of the normal modes, it is 
necessary to solve (3.81) using an iterative procedure described below:

1. Calculate $W_{j k \ell r}^{S}$ and $w_{j k \ell r}^{f}$ from initial conditions.

2. Set $W_{j k \ell r}^{f}$ to zero and calculate $P_{j k \ell}$.

3. Calculate $w_{j k \ell r}^{f}$ using (3.31).

4. Repeat step 3 with $P_{j k \ell r}$ calculated using new values of $w_{j k \ell r}^{f}$, until the iteration converges.

The above initialization procedure allows the model to be started smoothly for the case when the forcing term $Q$ is nonzero. The impact of the model initialization on the prediction of the track of a tropical cyclone will be discussed in chapter 6 .

\subsection{High Wavenumber Filtering}

As a tropical cyclone develops from a weak vortex the gradients of the dependent variables become very sharp near the center of the storm. This indicates that the Fourier representations of the dependent variables will have larger and larger amounts of energy in the higher wavenumbers. Since the Fourier series are truncated at a finite wavenumber, there is a tendency for the energy of the last few modes to become too large. This phenomenon, known as spectral blocking, is discussed in some detail by Machenhauer (1979) in the context of the one-dimensional nonlinear advection equation. Machenhauer indicates that a scale selective energy dissipation can be applied to reduce the errors caused by the blocking. In global models, linear diffusion terms of the form $K_{H} \nabla^{2}()$ or $K_{H} \nabla^{4}()$ have been added to the prognostic equations to inhibit blocking (Bourke, 1974; Simmons and Hoskins, 1978). When linear diffusion is added to the governing equations, it should prevent the model from developing scales of motion which are represented 
by the highest wavenumbers but should not affect the larger scales of motion. In order to get some idea of the effect of linear diffusion, consider the following diffusion problem:

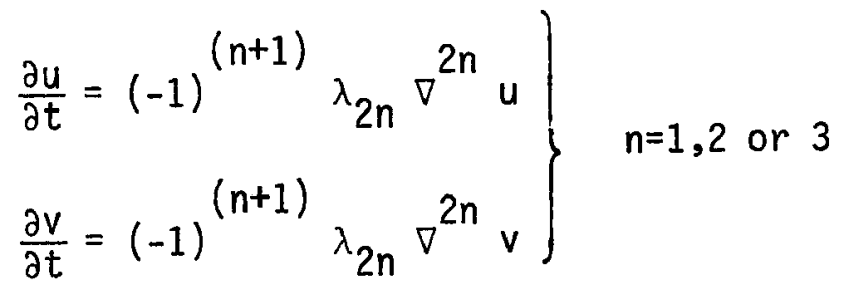

where $\lambda_{2 n}$ is the diffusion coefficient and $u$ and $v$ are the $x$ and $y$ components of the horizontal momentum. Equations (3.82) can be solved straightforwardly using the Galerk in method described in section 3.1. Applying the Galerkin procedure to $(3,82)$ gives the following equations for the Fourier series amplitudes of $u$ and $v$ :

$$
\left.\begin{array}{l}
\frac{\hat{d u}_{k \ell}}{d t}=-\lambda_{2 n}\left(k^{2}+l^{2}\right)^{n} \hat{u}_{k \ell} \\
\frac{d \hat{v}_{k \ell}}{d t}=-\lambda_{2 n}\left(k^{2}+l^{2}\right)^{n} \hat{v}_{k \ell}
\end{array}\right\} \text {. }
$$

The solutions of the above equations are given by

$$
\left.\begin{array}{l}
\hat{u}_{k \ell}(t)=\hat{u}_{k \ell}(0) e^{-\lambda 2 n\left(k^{2}+l^{2}\right)^{n} t} \\
\hat{v}_{k \ell}(t)=\hat{v}_{k l}(0) e^{-\lambda 2 n\left(k^{2}+l^{2}\right)^{n} t}
\end{array}\right\}
$$

where $\hat{u}_{k \ell}(0)$ and $\hat{v}_{k \ell}(0)$ are found from initial conditions. To determine the rate of energy dissipation, the kinetic energy over the whole domain is considered. Using Parseval's relation, the kinetic energy can be calculated from the Fourier series amplitudes as follows:

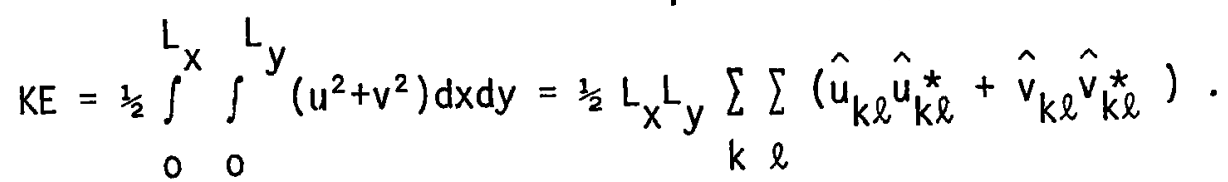


Now, consider the following initial condition:

$$
\left.\begin{array}{l}
u(x, y, 0)=A \sqrt{e} \frac{\left(y-y_{0}\right)}{r} \exp \left[-\frac{\left(x-x_{0}\right)^{2}+\left(y-y_{0}\right)^{2}}{2 r^{2}}\right] \\
v(x, y, 0)=-A \sqrt{e} \frac{\left(x-x_{0}\right)}{r} \exp \left[-\frac{\left(x-x_{0}\right)^{2}+\left(y-y_{0}\right)^{2}}{2 r^{2}}\right]
\end{array}\right\} .
$$

The above initial condition represents a vortex in the $x-y$ plane centered at the point $\left(x_{0}, y_{0}\right)$ with maximum wind speed $A$ at a radius $r$. For the case when the domain is much larger than $r$, the Fourier series amplitudes of (3.86) are given by

$$
\left.\begin{array}{l}
\hat{u}_{k \ell}(0)=\frac{2 \pi A \sqrt{e} i}{L_{x} L_{y}}(-1)^{m+n} r^{3} \ell e^{-r^{2} / 2\left(k^{2}+l^{2}\right)} \\
\hat{v}_{k \ell}(0)=-\frac{2 \pi A \sqrt{e} i}{L_{x} L_{y}}(-1)^{m+n} r^{3} k e^{-r^{2} / 2\left(k^{2}+l^{2}\right)}
\end{array}\right\} .
$$

The kinetic energy of the vortex defined by $(3.86)$ for the case when the domain is much larger than $r$ is given by

$$
K E(0)=\frac{\pi r^{2} A^{2} e}{2}
$$

Now, substituting (3.87) into (3.84) and then substituting these into (3.85) gives the kinetic energy of the vortex as a function of time. Normalizing the kinetic energy by the initial kinetic energy of the vortex (3.88) then gives

$$
K E(t)=\frac{4 \pi r^{4}}{L_{x} L_{y}} \sum_{k \ell} \sum_{l}\left(k^{2}+l^{2}\right) e^{-r^{2}\left(k^{2}+l^{2}\right)} e^{-2 \lambda 2 n\left(k^{2}+l^{2}\right)^{n} t} .
$$

Evaluating the above series gives the ratio of the kinetic energy of the 
vortex at time $t$ to the initial kinetic enrgy. Table 2 shows the amount of time it takes for the kinetic energy to decrease to half of its initial value for several size vorticies, several diffusion coeffictents and three forms of linear diffusion $(n=1,2$ and 3$)$. In a typical model simulation the physical space resolution on the transform grid is about $35 \mathrm{~km}$. The linear diffusion should then strongly damp this scale, but should not have too large an effect of scales larger than this. From Table 2 for second order diffusion $(n=1)$ it can be seen that if the diffusion coefficient is chosen so that it damps the $r=50 \mathrm{~km}$ vortex in less than a day, it also will damp the larger scales. For this reason it appears that higher order diffusion should be used. Both the fourth $(n=2)$ and sixth order diffusion $(n=3)$ were tested in the model and both were capable of reducing the effect of spectral blocking. Since the sixth order diffusion is more scale selective than the fourth order diffusion, the sixth order diffusion with $\lambda_{6}=10^{22} \mathrm{~m}^{6} \mathrm{~s}^{-2}$ was used in all of the model simulations discussed in the remaining chapters. Thus, terms of the form $\lambda_{6} \nabla^{6}()$ were added to the prognostic equations (2.38)(2.39), (2.41)-(2.42) and (2.47). As can be seen from Table 2, this prevents the formation of a vortex with a radius of maximum wind smaller than about $50 \mathrm{~km}$. 
TABLE 2.

Time in hours for linear diffusion of the form $\lambda_{2 n} \nabla^{2 n}()$ to halve the kinetic energy of a Gaussian type vortex with radius of maximum wind $r$.

\begin{tabular}{|c|c|c|c|c|c|}
\hline & & $r=50 \mathrm{~km}$ & $r=100 \mathrm{~km}$ & $r=150 \mathrm{~km}$ & $r=200 \mathrm{~km}$ \\
\hline$n=1$ & $\lambda_{2}=10^{3} \mathrm{~m}^{2} \mathrm{~s}^{-1}$ & $144 \mathrm{hrs}$ & $575 \mathrm{hrs}$ & $1290 \mathrm{hrs}$ & $2300 \mathrm{hrs}$ \\
\hline$\left(\lambda_{2} \nabla^{2}\right)$ & $\lambda_{2}=10^{4} \mathrm{~m}^{2} \mathrm{~s}^{-1}$ & $14.4 \mathrm{hrs}$ & $57.5 \mathrm{hrs}$ & $129 \mathrm{hrs}$ & $230 \mathrm{hrs}$ \\
\hline$n=2$ & $\lambda_{4}=10^{13} \mathrm{~m}^{4} \mathrm{~s}^{-1}$ & $20.1 \mathrm{hrs}$ & $322 \mathrm{hrs}$ & $1631 \mathrm{hrs}$ & $5160 \mathrm{hrs}$ \\
\hline$\left(\lambda_{4} \nabla^{4}\right)$ & $\lambda_{4}=10^{14} \mathrm{~m}^{4} \mathrm{~s}^{-1}$ & $2.01 \mathrm{hrs}$ & $32.2 \mathrm{hrs}$ & $163 \mathrm{hrs}$ & $516 \mathrm{hrs}$ \\
\hline$n=3$ & $\lambda_{6}=10^{22} \mathrm{~m}^{6} \mathrm{~s}^{-1}$ & $28.8 \mathrm{hrs}$ & $1840 \mathrm{hrs}$ & $21000 \mathrm{hrs}$ & $118000 \mathrm{hrs}$ \\
\hline$\left(\lambda_{6} \nabla^{6}\right)$ & $\lambda_{6}=10^{23} \mathrm{~m}^{6} \mathrm{~s}-1$ & $2.88 \mathrm{hrs}$ & $184 \mathrm{hrs}$ & $2100 \mathrm{hrs}$ & $11800 \mathrm{hrs}$ \\
\hline
\end{tabular}


CHAPTER 4

NUMER ICAL EXAMPLES

In this chapter four numerical integrations of the tropical cyclone model described in chapters 2 and 3 are presented. These simulations as well as the values of the numerical parameters used are summarized in Table 3. Unless specified in Table 3, the values of the physical parameters used are those which are listed in Table 1. In each of the four simulations the Fourier series were truncated at $M=N=35$ and the high wavenumber filter described in section 3.6 was applied. The grid spacing of the transform grid used to calculate the nonlinear terms is listed in Table 3 as the physical space grid resolution. A five day model integration with a $90 \mathrm{sec}$ time step required about 30 minutes of computing time on the NCAR Cray-1A computer.

Simulations similar to $4 A$ and $4 D$ in Table 3 have been presented by Ooyama (1969a) and Bliss (1980). These simulations using the current model are compared to the previous work in sections 4.1 and 4.4. Simulation $4 B$ is designed to test the effect of $\beta$ on the development of a tropical cyclone and simulation $4 C$ shows how the motion of a tropical cyclone can affect its structure.

\subsection{Simulation of an Axisymmetric Tropical Cyclone on an f-plane}

In this section numerical results from a five day model integration with an axisymmetric initial condition and $\beta=0$ are presented. The 
TABLE 3.

Summary of the numerical simulations

presented in chapter 4

\begin{tabular}{|c|c|c|c|c|c|c|}
\hline & Initial Condition & $\begin{array}{l}\text { Domain } \\
\text { Size }(\mathrm{km})\end{array}$ & $\begin{array}{l}\text { Physical Space } \\
\text { Grid Resolution }(\mathrm{km})\end{array}$ & $\begin{array}{c}\text { Time } \\
\text { Step (sec) }\end{array}$ & $\begin{array}{l}\text { Integration } \\
\text { length (days) }\end{array}$ & $\begin{array}{l}\text { Corolis } \\
\text { parameter }\end{array}$ \\
\hline $4 A$ & $\begin{array}{l}\text { Symmetric vortex in } \\
\text { layers } 0 \text { and } 1 \\
v_{m}=10 \mathrm{~ms}^{-1} r_{\mathrm{m}}=100 \mathrm{~km}\end{array}$ & $3600 \times 3600$ & 33 & 90 & 5 & $\beta=0$ \\
\hline $4 B$ & $\begin{array}{l}\text { Symmetric vortex in } \\
\text { layers } 0 \text { and } 1 \\
V_{m}=10 \mathrm{~ms}^{-1} r_{\mathrm{m}}=100 \mathrm{~km}\end{array}$ & $3600 \times 3600$ & 33 & 90 & 4 & $\beta \neq 0$ \\
\hline $4 C$ & $\begin{array}{l}\text { Symmetric vortex in } \\
\text { layers } 0 \text { and } 1 \text { with } \\
\text { basic state zonal } \\
\text { wind } \\
v_{m}=10 \mathrm{~ms}^{-1} r_{m}=150 \mathrm{~km}\end{array}$ & $3600 \times 3600$ & 33 & 90 & 5 & $\beta \neq 0$ \\
\hline $4 \mathrm{D}$ & $\begin{array}{l}\text { Wave- } 1 \text { ike distur- } \\
\text { bance in layers } 0 \\
\text { and } 1 \text { with basic } \\
\text { state zonal wind }\end{array}$ & $4000 \times 4000$ & 37 & 120 & 6 & $\beta \neq 0$ \\
\hline
\end{tabular}


purpose of this simulation is to demonstrate that the current model can produce an axisymmetric tropical cyclone similar to storms observed in nature and also to compare the current model to the original work of Ooyama (1969a). If the governing equations were solved exactly on an infinite domain with $\beta=0$ an initial axisymmetric vortex should remain axisymmetric for all time. The magnitude of the asymmetries which develop should then give some idea of the effect of the periodic boundaries and the use of a numerical approximation scheme in cartesian geometry.

For this simulation the model is initialized with a vortex of the form

$$
V(r)=v_{m}\left(\frac{r}{r_{m}}\right) \exp \left(1-r / r_{m}\right)
$$

in layers 0 and 1 , with layer 2 at rest. In (4.1) the maximum tangential wind $V_{m}$ is specified to be $10 \mathrm{~ms}^{-1}$ at a radius $r_{m}$ of $100 \mathrm{~km}$. The initial vortex given by $(4.1)$ is somewhat different than the one used by Ooyama. Ooyama's initial vortex decayed like $1 / r$ for large radii. In the current model it is necessary for the vortex profile to decay more rapidly at larger radius in order to avoid a discontinuity at the boundary. Since $V$ decays exponentially for large $r$ in (4.1), $V$ is negligible at the boundary when the vortex is centered on a 3600 by 3600 $\mathrm{km}$ domain. Although the vortex for this simulation decays more rapidly than 0oyama's vortex at large radii, it is larger at smaller radii (Ooyama's initial vortex had a maximum wind of $10 \mathrm{~ms}^{-1}$ at a radius of 50 $\mathrm{km})$. Since Ooyama's model was axisymmetric and balanced it was possible for the spatial resolution to be very fine (0oyama used a $\Delta r$ of $5 \mathrm{~km}$ in his finite difference mode1). As discussed in section (3.6) the 
smallest vortex the current model can resolve has a radius of maximum wind of about $50 \mathrm{~km}$ so that it was necessary to use a larger initial vortex.

Since the streamfunction and velocity potential are used in place of horizontal velocity components, (4.1) cannot be used directly for initialization. The vorticity of the initial vortex as a function of $r$ is given by

$$
\zeta(r)=\frac{2 V_{m} e}{r_{m}}\left[1-\frac{1}{2}\left(\frac{r}{r_{m}}\right)\right] e^{-r / r_{m}} .
$$

Using the relation

$$
r=\left[\left(x-x_{0}\right)^{2}+\left(y-y_{0}\right)^{2}\right]^{\frac{1}{2}}
$$

where $\left(x_{0}, y_{0}\right)$ are the coordinates of the vortex center, (4.2) can be used to calculate the vorticity at the physical space grid points defined by (3.28). Once this is done, trapezodial quadrature of the form of (3.27) can be used to find the spectral coefficients $\hat{\zeta}_{k \ell}$ from the grid point values. The spectral coefficients of the streamfunction can then be found from the transformed diagnostic relation (3.22). The initial vortex is assumed to be nondivergent so that the spectral coefficients of the velocity potential are set to zero.

Once the wind field is specified, it is necessary to calculate $\phi_{1}$ and $\phi_{2}$ in a consistent way. Since the upper level is assumed to be at rest, $\phi_{2}$ is set to zero. For the axisymmetric case $\phi_{1}(r)$ could be calculated by substituting (4.1) into the gradient wind equation and then integrating with respect to $r$. The spectral coefficients of $\phi_{1}$ could then be found similar to the calculation of $\hat{\zeta}_{i k \ell}$. Since this can not be done for an asymmetric initial condition, a more general 
procedure is used. If it is assumed that initially the divergence and the time tendency of the divergence are zero, the divergence equation (2.39) for layer $i$ reduces to

$$
\nabla^{2} \phi_{i}=f_{0} \zeta_{i}-\frac{\partial}{\partial y}\left(u_{i} \zeta_{i}\right)+\frac{\partial}{\partial x}\left(v_{i} \zeta_{j}\right)-\nabla^{2}\left(\frac{u_{i}^{2}+v_{i}^{2}}{2}\right)
$$

which is the nonlinear balance equation. Applying the Galerkin procedure to the above equation and using the transformed diagnostic equation (3.22) gives

$$
\hat{\phi}_{i k \ell}=f_{0} \hat{\psi}_{i k \ell}-\left(k^{2}+\ell^{2}\right)^{-1}\left[-i \ell \hat{A}_{i k \ell}+i k \hat{B}_{i k \ell}+\left(k^{2}+\ell^{2}\right) \hat{E}_{i k \ell}\right]
$$

where $A_{i}, B_{i}$ and $E_{i}$ are defined by (3.21). The transform of the nonlinear terms $A_{i}, B_{i}$ and $E_{i}$ can be computed using the transform method described in section (3.2) so that the above equation can be used to calculate $\hat{\phi}_{i k \ell}$ for layer 1 , given $\hat{\psi}_{i k \ell}$.

The remaining dependent variable to be specified is the equivalent potential temperature deviation $\Lambda_{0}$. For simplicity, it is assumed that initally $\Lambda_{0}$ has a constant value of $10 \mathrm{~K}$ which corresponds to a $\theta_{\mathrm{e}}$ of about $350 \mathrm{~K}$.

Since the numerical model predicts the amplitudes of the Fourier series it is possible to calculate the dependent variables at any given point in the domain. For the axisymmetric vortex in experiment $4 \mathrm{~A}$ the dependent variables were calculated on a cylindrical grid centered on the vortex (at $x=y=1800 \mathrm{~km}$ ) for display purposes. The azimuthal average of the dependent variables using eight values at each radial point was then calculated in order to compare the current model results to Ooyama's axisymmetric model. The tangential wind relative to the 
cylindrical coordinate system (defined at VT) was calculated to simplify the model comparison. The standard deviation $\sigma$ of the dependent variables from the azimuthal mean using the eight values at each radial point was also calculated to test the ability of the model to remain axisymmetric during an integration.

Fig. 5 shows the azimuthal averages of the layer 1 and 2 tangential winds VT1 and VT2, the layer 1 and 2 geopotentials $\phi_{1}$ and $\phi_{2}$, the vertical velocity at the top of the boundary layer $w$ and the convective stability parameter $n$ at $0,24,48,72,96$ and 120 hours. During the 5 day integration the layer 1 tangential wind increases from $10 \mathrm{~ms}^{-1}$ at a radius of $100 \mathrm{~km}$ to about $50 \mathrm{~ms}^{-1}$ at a radius of $60 \mathrm{~km}$. The layer 2 tangential circulation which develops is cyclonic inside a radius of about $300 \mathrm{~km}$ with a much larger anticyclonic circulation outside of 300 $\mathrm{km}$. The cyclonic circulation which develops in layer 2 is largely due to the transport of the layer 1 momentum by the diabatic term $Q$. Consistent with the tangential winds, the radial gradient of the geopotential deviation is positive where the wind is cyclonic and negative where the wind is anticyclonic.

The surface pressure $P_{S}$ in the incompressible fluid system is given by

$$
P_{s}=\rho g\left[H_{0}+H_{1}+h_{1}+\varepsilon\left(H_{2}+h_{2}\right)\right] .
$$

It is convenient to consider the deviation from the mean surface pressure $P_{S}{ }^{\prime}$ where the mean surface pressure is the value of $P_{S}$ when $h_{1}=h_{2}=0$. Dropping the prime, the surface pressure deviation is given by

$$
P_{s}=\rho g\left(h_{1}+\varepsilon h_{2}\right)=\rho \phi_{1} \text {. }
$$



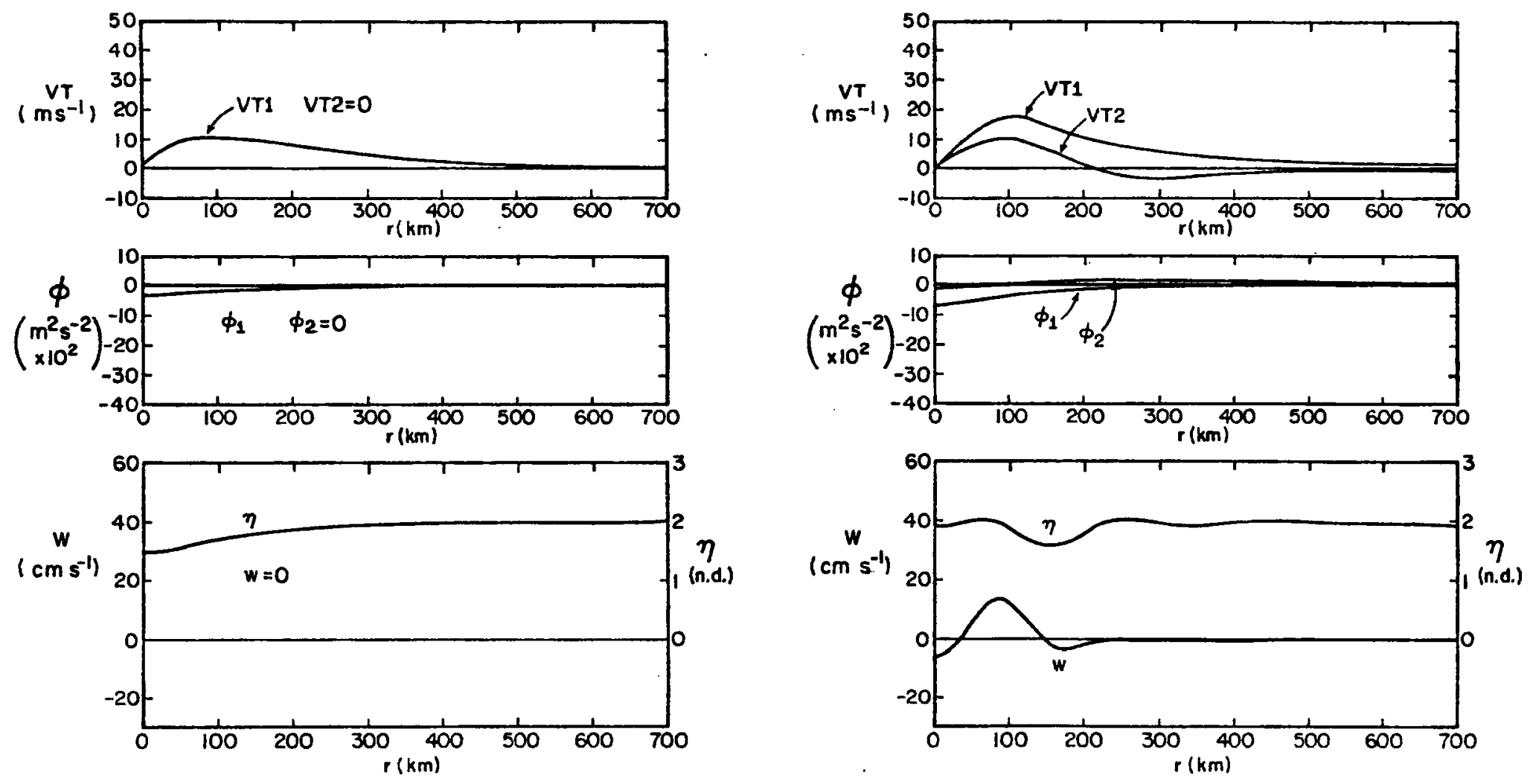

(a) $t=0$

(b) $t=24 \mathrm{hrs}$

Figure 5. The azimuthally averaged tangential wind VT and geopotential $\phi$ for layers 1 and 2 , vertical velocity at the top of the boundary layer $w$ and vertical stability parameter $r$ as a function of radius $r$ for experiment $4 A$. The fields at $0,24,48,72,96$ and 120 hours are shown in $(a)-(f)$ : 

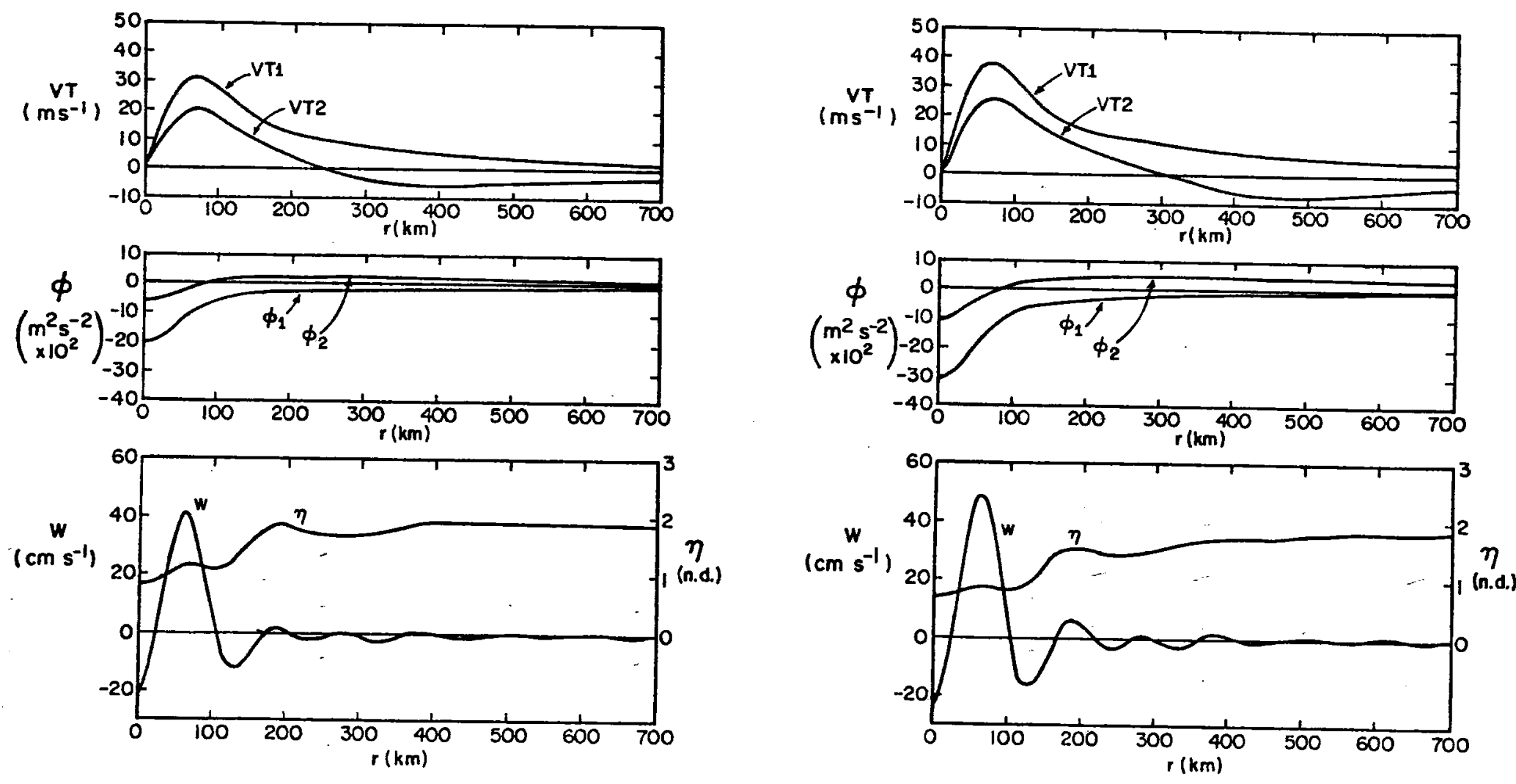

(c) $t=48 \mathrm{hrs}$

(d) $t=72 \mathrm{hrs}$

Figure 5. (continued) 

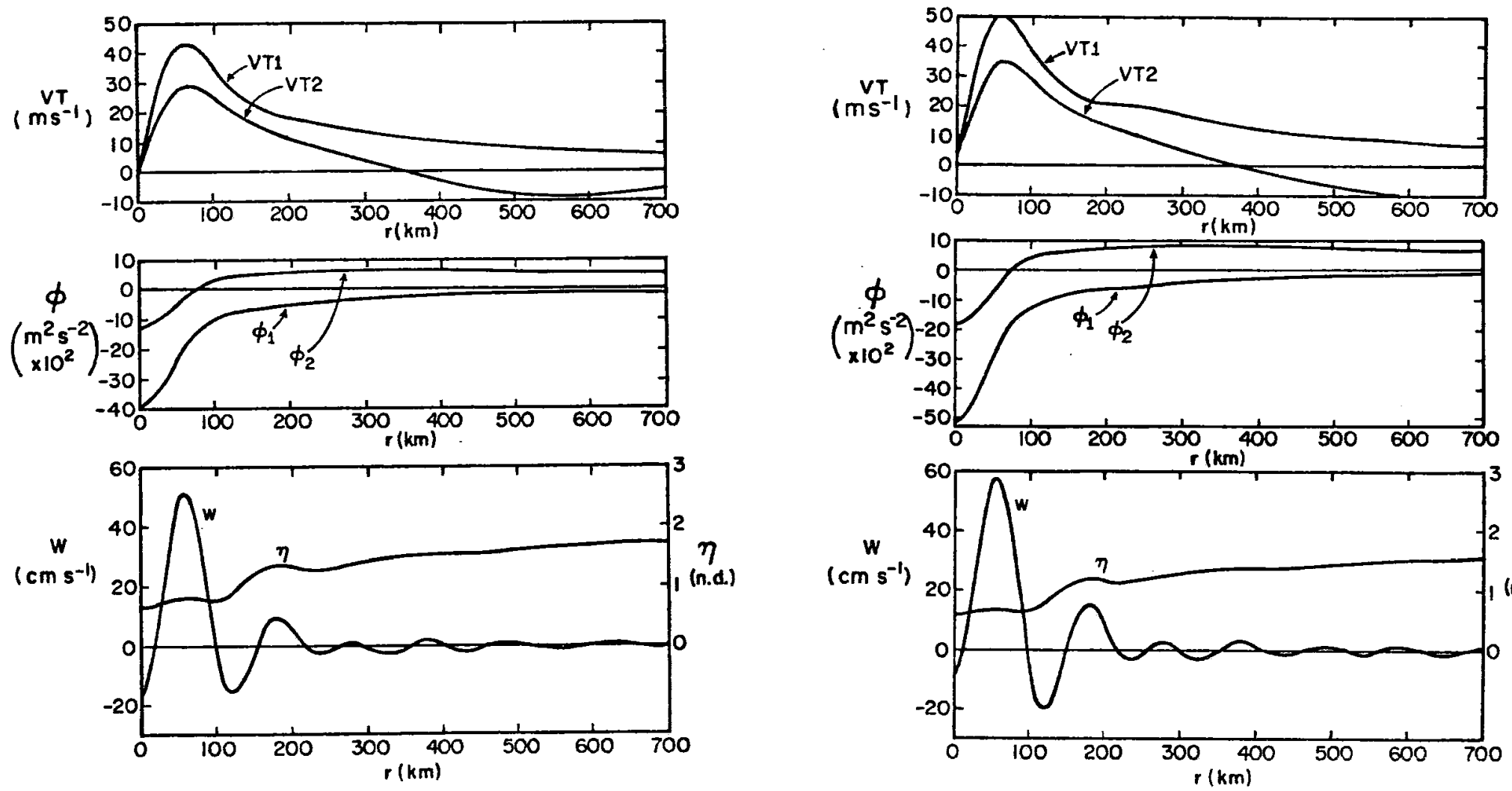

(e) $t=96 \mathrm{hrs}$

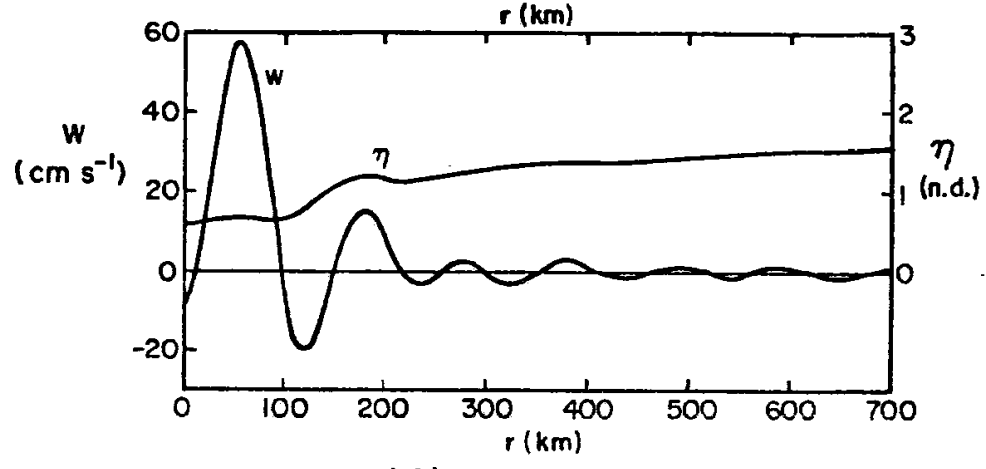

Figure 5. (continued) 
Since the density. of layer 1 is approximately equal to $1 \mathrm{~kg} \mathrm{~m}^{-3}$, the layer 1 geopotential can be interpreted in terms of a surface pressure deviation. For example, a $\phi_{1}$ value of $100 \mathrm{~m}^{2} \mathrm{~s}^{-2}$ corresponds to a surface pressure deviation of $100 \mathrm{~Pa}$, or $1 \mathrm{mb}$. Using (4.7) it can be seen in Fig. 5 that the surface pressure deviation decreases from about $-3 \mathrm{mb}$ to $-50 \mathrm{mb}$ during the 5 day simulation. Assuming a mean surface pressure of about $1010 \mathrm{mb}$, the model tropical cyclone would have a minimum surface pressure of $960 \mathrm{mb}$. Shea and Gray (1973) have studied the inner structure of Atlantic hurricanes. They have shown that hurricanes with a central pressure of $960 \mathrm{mb}$ can have maximum tangential winds ranging from 30 to $55 \mathrm{~ms}^{-1}$. They have also shown that hurricanes with $50 \mathrm{~ms}^{-1}$ winds can have a radius of maximum wind ranging from about 10 to $70 \mathrm{~km}$. Thus, the inner region of the model tropical cyclone is similar to some of the larger storms observed in nature.

In the tropical cyclone simulation shown in Fig. 5, the layer 1 tangential wind outside of $100 \mathrm{~km}$ also increases as the storm intensifies. By 120 hours the radius of $15 \mathrm{~ms}^{-1}$ winds is about $325 \mathrm{~km}$ and the radius of $10 \mathrm{~ms}^{-1}$ winds is about $475 \mathrm{~km}$. Frank and Gray (1980) have presented composite $850 \mathrm{mb}$ tangential wind profiles from 10 years of northwest Pacific typhoon rawinsondes and 14 years of West Indies hurricane rawinsondes. These results show that for storms with maximum tangential wind speeds between 41 and $51 \mathrm{~ms}^{-1}$, the radii of $15 \mathrm{~ms}^{-1}$ and $10 \mathrm{~ms}^{-1}$ winds are found at about $300 \mathrm{~km}$ and $550 \mathrm{~km}$ for the composite hurricane and at about $400 \mathrm{~km}$ and $700 \mathrm{~km}$ for the composite typhoon. The model produced tropical cyclone at large radii is then slightly weaker than, but similar to tropical cyclones observed in nature. Frank (1977) has shown that $850 \mathrm{mb}$ is approximately the level of maximum winds for 
typhoons. Since the layer 1 tangential wind is probably more representative of the average tangential wind between about $900 \mathrm{mb}$ and $500 \mathrm{mb}$, the radius of the $15 \mathrm{~ms}^{-1}$ and $10 \mathrm{~ms}^{-1}$ winds in the model should be expected to somewhat smaller than those observed.

The lower portion of Fig. 5 shows the vertical velocity at the top of the boundary layer $w$ and the convective stability parameter $n$. There is no vertical velocity at $t=0$ since the initial condition was nondivergent. By 24 hours an area of rising motion has developed between about $30 \mathrm{~km}$ and $120 \mathrm{~km}$ due to the boundary layer friction, with the maximum at $85 \mathrm{~km}$. The diabatic term $Q$ is equal to $n \mathrm{w}$ when $w$ is positive so that the maximum value of $Q$ is located very near the maximum value of w. In Fig. 5b, the maximum diabatic heating occurs just inside the radius of maximum wind which then causes the radius of maximum wind to contract as the storm develops. In Figs. $5 c-5 f$, the maximum value of $w$ remains inside the radius of maximum wind as the storm intensifies. The contraction of the radius of maximum wind during tropical cycione intensification has also been observed in nature. Willoughby et al. (1982) have presented several examples of this contraction during intensification of strong symmetric tropical cyclones.

In the lower portion of Fig. 5 it can be seen that two areas of negative vertical velocity develop in addition to the main area of positive $w$. A very small area of negative $w$ is found near the center of the vortex as well as a larger area just outside of the main vertical motion maximum. The relatively strong subsidence located between about 100 and $160 \mathrm{~km}$ in Fig. $5 c-5 \mathrm{f}$ would have a tendency to suppress convection in this area. A feature similar to this can sometimes be observed in storms found in nature. Fig. 6 from Willoughby et al. 


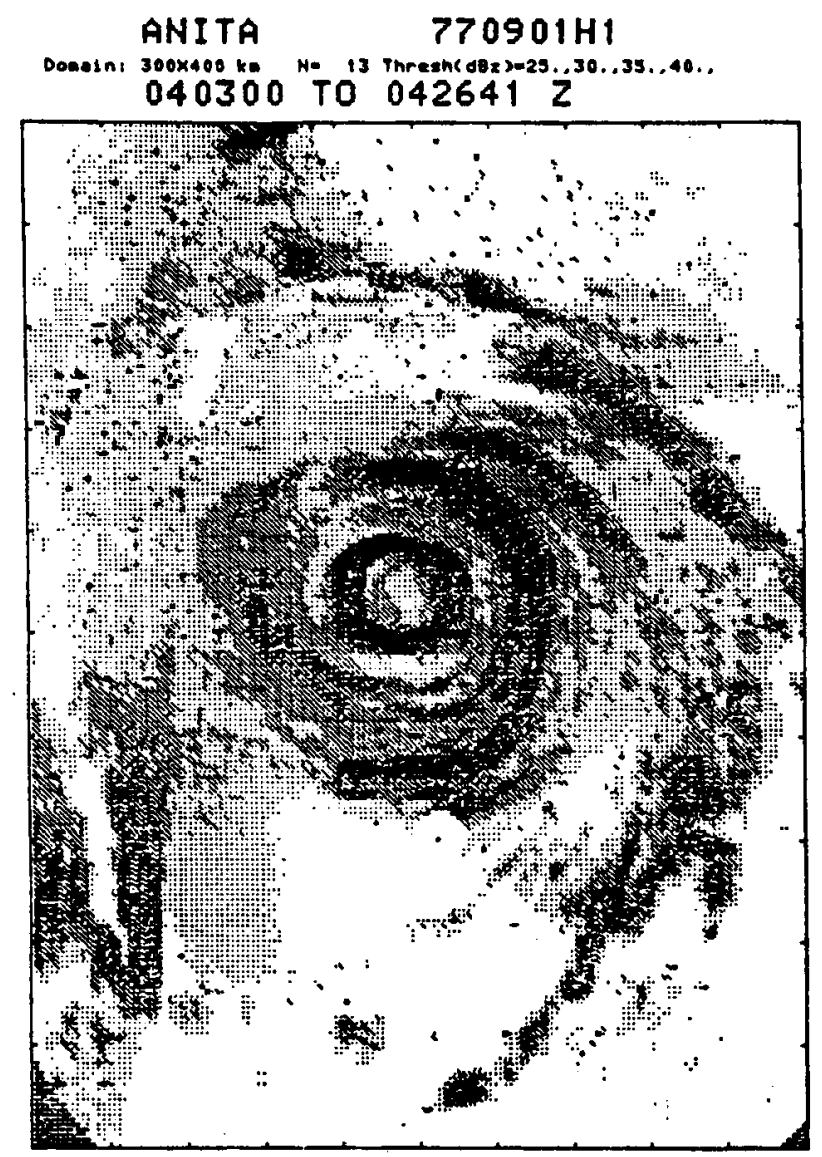

Figure 6. A digital radar reflectivity composite of hurricane Anita from 2 September 1977 (from Willoughby et al., 1982). 
(1982) shows a digital radar reflectivity composite of hurricane Anita from 2 September 1977. In Fig. 6 a ring of low reflectivity occurs just outside of the intense convection of the eye wall.

After 24 hours in Fig. 5 the vertical velocity oscillates outside a radius of about $200 \mathrm{~km}$. This oscillation is probably due to lack of enough Fourier modes to fully resolve the sharp vertical velocity peak which occurs inside $100 \mathrm{~km}$. The first part of this oscillation with a maximum at a radius of $180 \mathrm{~km}$ in Fig. $5 \mathrm{~d}$ may, however, be a real feature of the model. The small maximum in $w$ at $t=72$ hours increases after this time, and by 120 hours has reached $15 \mathrm{cms}^{-1}$. At $t=120$ hours it appears that a secondary maximum in the layer 1 tangential wind is starting to form at about $220 \mathrm{~km}$ in association with the secondary maximum in the boundary layer vertical velocity. This feature is somewhat similar to (on a larger scale) the concentric eye wall structure which has been reported by Jordan and Schatzle (1961) and more recently by Willoughby et al. (1982). Fig. 6 is an example of a storm with concentric eye walls. This indicates that it may be possible to use the simplified physics of the current model to gain some insight into the formation of concentric eye walls and secondary wind maxima.

The convective stability parameter $\eta$ is also shown in Fig. 5 . As can be seen in (2.44), $n$ is a function of the equivalent potential temperature $\Lambda_{j}$ of each of the model layers, where $\Lambda_{0}$ is predicted by (2.47), $\Lambda_{1}$ is held constant and $\Lambda_{2}$ is diagnosed from (2.45). The value of $\eta$ in (2.44) increases as $\Lambda_{0}$ increases and decreases as $\Lambda_{2}$ increases (or as $h_{2}$ increases in 2.45). At $t=0$ in Fig. $5 n$ decreases towards the center of the vortex. This is caused by the initial low value of $\phi_{1}$ at the center of the vortex since $h_{2}$ is proportional to $\phi_{2}-\phi_{1}$. By $t=24$ 
hours $n$ has increased near the center of the vortex and a minimum has formed near $r=150 \mathrm{~km}$. The increase in $n$ near the center of the vortex is caused by the increase in $\Lambda_{0}$ due to the large surface flux in the region of the large surface winds. The low value of $n$ is caused by a decrease of $\Lambda_{0}$ due to the subsidence near $r=170 \mathrm{~km}$. The magnitude of the effect of the subsidence can be estimated from the vertical advection term in (2.47) (third term on the right). Assuming $\Lambda_{0}=10 \mathrm{~K}$, $\Lambda_{1}=-10 \mathrm{~K}$ and $\mathrm{w}^{-}=3 \mathrm{~cm} \mathrm{~s} \mathrm{~m}^{-1}$, this term has a magnitude of about $-50 \mathrm{~K}$ per day. The value of $\Lambda_{0}$ does not change by this much since this tendency is balanced by the other terms in (2.47), but the subsidence does cause $n$ to have a minimum in this region. By $t=48$ hours the value of $n$ has decreased inside about $100 \mathrm{~km}$. This is partially caused by the lowered values of $\Lambda_{0}$ due to the subsidence, but also is a result of the increase in $h_{2}$. As the storm intensifies further, $n$ continues to decrease, so that the magnitude of the diabatic term $Q$ decreases. It is this decrease in $n$ which eventually slows the intensification of the model tropical cyclone.

Some of the weaknesses of the model can be seen by looking at the parameter $n$. By $t=72$ hours in Fig. 5 ; the value of $n$ is less than one inside a radius of about $100 \mathrm{~km}$ and continues to decrease after this time. If the model continued to run past 5 days, it would be possible for $n$ to become negative, which would correspond to negative diabatic heating by the cumulus clouds. As discussed previously, the low values of $\eta$ are caused by the decrease in $\Lambda_{0}$ due to subsidence and to the increase in $\lambda_{2}$ due to the increase in the upper level thickness $h_{2}$. The increase in $h_{2}$ near the center of the cyclone is analogous to the formation of a warm core in a compressible fluid system, which should be 
expected to decrease the diabatic heating term. Since the periodic doma in is closed, however, the average value of $h_{2}$ also increases during the model integration so that the value of $n$ decreases everywhere. In a compressible system on a closed finite domain, this is equivalent to a net heating of the model atmosphere. This indicates that the domain size limits the length of time the model can be integrated. For this reason the model integrations were limited to 6 days or less.

The decrease in $n$ due to the subsidence is caused by the vertical advection term in (2.47). This represents a real physical process, but it appears that the approximation of a constant $\Lambda_{1}$ leads to an overestimation of the decrease of $\Lambda_{0}$ due to the vertical advection. In a tropical cyclone, the equivalent potential temperature of the atmosphere between $900 \mathrm{mb}$ and $500 \mathrm{mb}$ in the inner regions of the storm is about 5 to $10 \mathrm{~K}$ warmer than that of the mean tropical atmosphere (Sheets, 1969). Thus, if 1 were predicted in a more realistic way, the magnitude of the vertical advection would be reduced as $\Lambda_{1}$ increased.

The tropical cyclone simulation shown in Fig. 5 is somewhat similar to the simulation presented by 0oyama (1969a). The current model uses 0oyama's incompressible fluid system and cumulus parameterization scheme, and both models were run with the same sea surface temperature. The initial conditions, however, are different since the initial vortex used here has a radius of maximum wind which is twice as large as used by 0oyama and has a vortex profile which decays more rapidly at large radii. Another difference is that the current model uses primitive equations, while 0oyama's model was balanced so that gravity waves were filtered. The computational procedures also differed considerabiy since Ooyama's model was solved using finite difference methods on an open 
domain while the current model was solved using the Galerkin method on a larger doubly-periodic domain. Despite these differences, the simulation from Ooyama (1969a) shown in Fig. 7 is similar to the simulation shown in Fig. 5. The general structure of the tangential winds and geopotential fields from Ooyama's model at $t=162$ hours are very similar to those shown in Fig. $5 f$.

Comparing Fig. $7 \mathrm{a}$ with Fig. $5 \mathrm{c}$ it can be seen that the tropical cyclone in the current model develops more rapidly than in 0oyama's model. As pointed out by 0oyama (1969b), this is caused by the use of the balance approximation in the boundary layer. When this assumption was relaxed in 0oyama's later work, the tropical cyclone intensified much more rapidly.

Although the tangential wind and geopotential profiles are similar in Figs. 5 and 7 , the vertical velocity $w$ and convective stability profiles are quite different. Comparing Figs. 5e or $5 \mathrm{f}$ with Fig. $7 \mathrm{~b}$ it can be seen that the maximum vertical velocity is about 5 times larger for 0oyama's model when the two simulations had comparable maximum winds. This difference is probably due to the greater resolution used by Ooyama (Ooyama's grid spacing was $5 \mathrm{~km}$ compared to the $33 \mathrm{~km}$ resolution on the transform grid in the current model). In addition to the amplitude differences, the structure of the $w$ profiles are also somewhat different. The subsident area found between about 100 and 150 $\mathrm{km}$ in Fig. 5 is much less well defined in 0oyama's model results. There is some evidence of a subsident area near $100 \mathrm{~km}$ in Figs. $7 \mathrm{a}$ and $7 \mathrm{~b}$, but the amplitude is smaller. This difference is probably related to the use of balanced versus primitive equations, although further study is necessary to resolve this question. Another difference between the two 

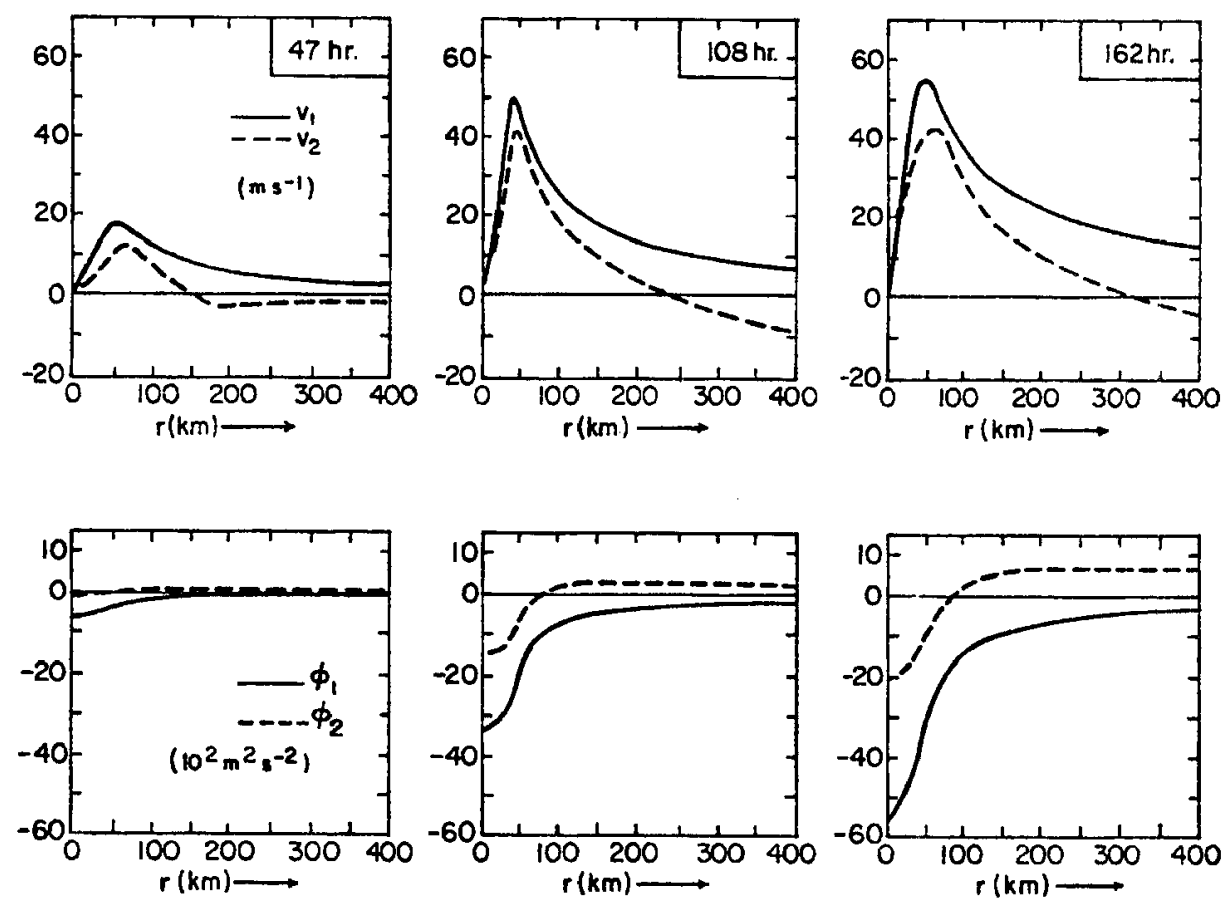

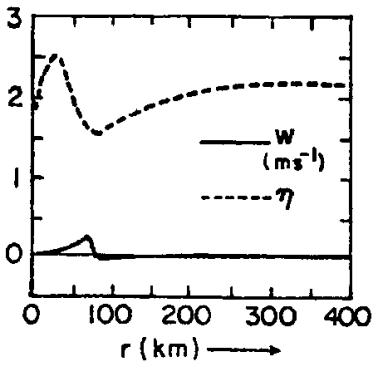

(a)

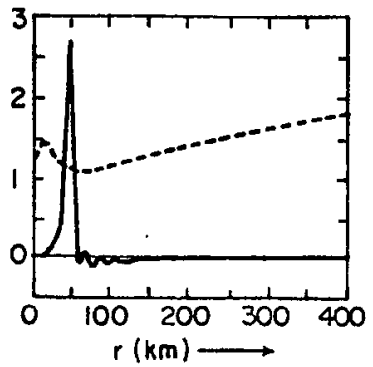

(b)

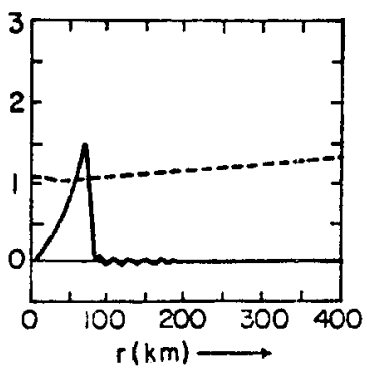

(c)

Figure 7. The tangential wind $V$ and geopotential $\phi$ for layers 1 and 2, vertical velocity at the top of the boundary layer $w$ and vertical stability parameter $n$ as a function of radius $r$ from the axisymmetric balanced tropical cyclone model of 0oyama (1969a). The fields at 47, 108 and 162 hours are shown in (a)-(c) respectively. 
simulations is that the maximum vertical velocity in Fig. 7 occurs outside of the radius of maximum wind, which, as pointed out by 0oyama (1969b), tends to cause the vortex to expand unrealistically. When the assumption of gradient wind balance in the boundary layer was relaxed in Ooyama's later work, the vertical velocity maximum occurred inside the radius of maximum wind, similar to the results shown in Fig. 5 for the current model. Comparing Fig. 5 after $t=24$ hours with Fig. $7 \mathrm{~b}$ and $7 \mathrm{c}$ it can be seen that the convective stability parameter $\eta$ is smaller inside about $100 \mathrm{~km}$ for the current mode1. This is caused by the subsidence between about 100 and $150 \mathrm{~km}$ as well as by the effect of the convection in a closed domain as discussed previously.

In summary, the current model is capable of producing an axisymmetric tropical cyclone which is similar to those found in nature. The model can also reproduce some of the results presented by 0oyama $(1969 a, b)$. The differences between the two models result from the use of primitive equations in the current model, and the differing horizontal resolution. The use of a closed domain in the current model appears to limit a tropical cyclone simulation to about six days.

All of the results show in Fig. 5 are azimuthal averages of the dependent variables. Fig. $8 b$ shows the standard deviation $\sigma$ from the eight point azimuthal average of the dependent variables at $t=96$ hours as a function of radius. The average values at $t=96$ hours are shown in Fig. 8a for comparison. Fig. 8b shows that the standard deviations of the tangential wind for layers 1 and 2 are less than about $0.2 \mathrm{~ms}^{-1}$ and $0.3 \mathrm{~ms}^{-1}$ respectively inside a radius of $700 \mathrm{~km}$. From Fig. $8 \mathrm{a}$ it can then be seen that the azimuthally averaged tangential winds are about two orders of magnitude larger than the standard deviations. Similarly, 

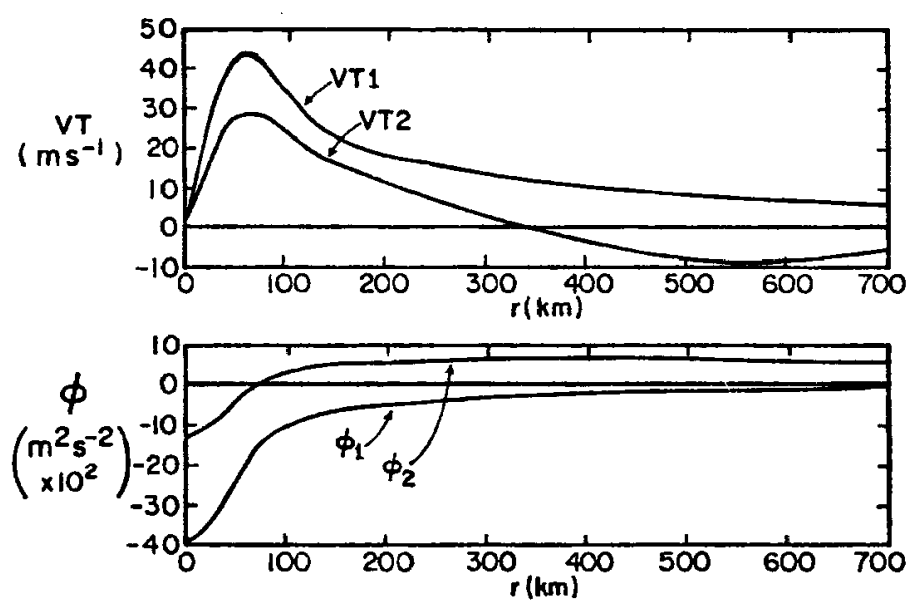

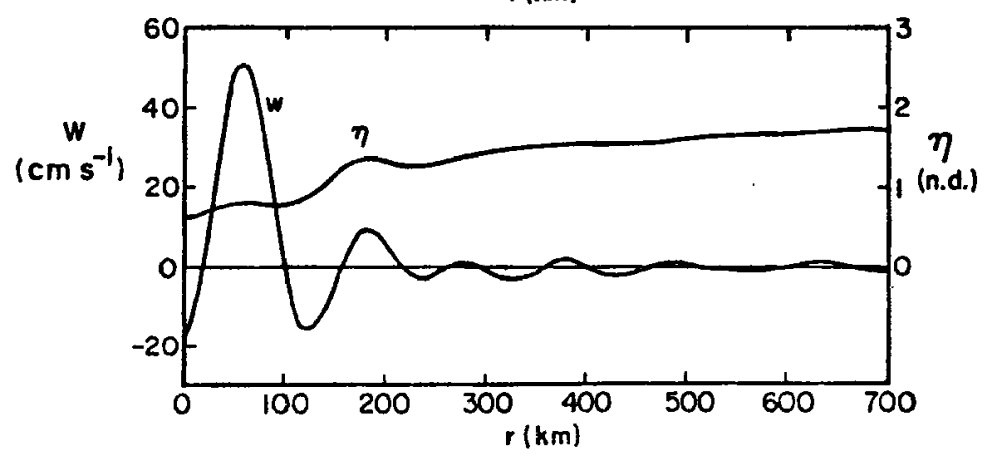

(a)

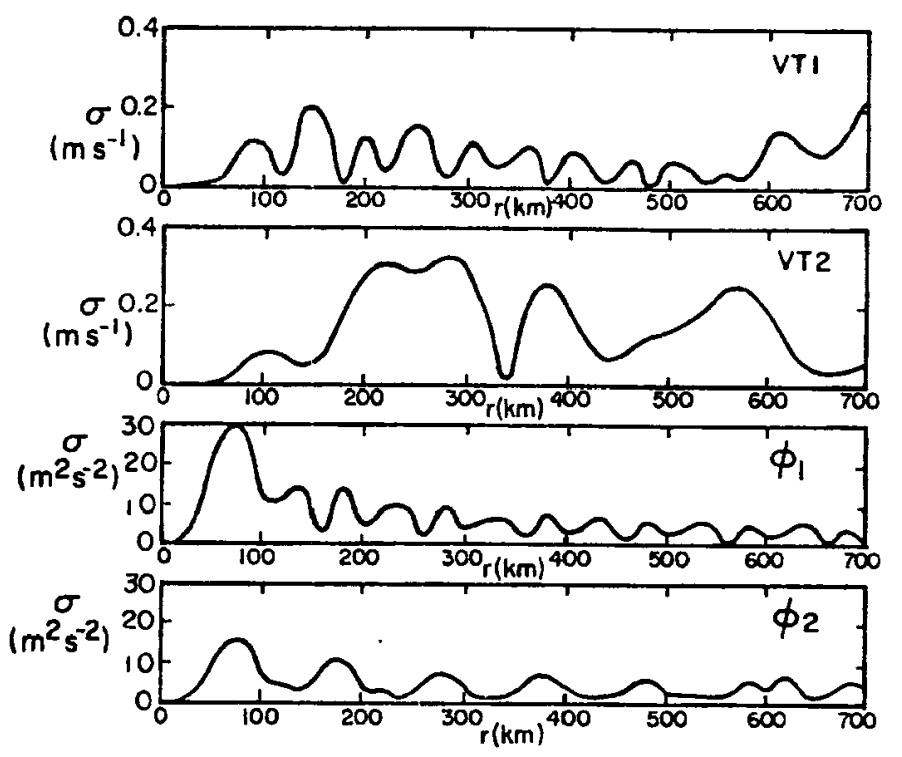

Figure 8. The standard deviation $\sigma$ from the azimuthal average of the tangential wind VT and geopotential $\phi$ for layers 1 and 2 and the vertical velocity at the top of the boundary layer $w$ as a function of radius $r$ for experiment 4A at 96 hours (b). The azimuthal average values at 96 hours are shown in $(a)$. 
the azimuthally averaged geopotentials for layers 1 and 2 are also about two orders of magnitude larger than the corresponding standard deviations. These results indicate that after 96 hours the vortex has remained axisymmetric inside a radius of $700 \mathrm{~km}$. Thus, the use of cartesian geometry and a doubly-periodic domain does not appear to introduce artificial asymmetries in the wind and geopotential fields.

The lower portion of Fig. 8b shows the standard deviation of the vertical velocity field from the azimuthal average. From Fig. 8 a it can be seen that the standard deviation is about a factor of 10 smaller than the mean inside $100 \mathrm{~km}$, a factor of 3 smaller between 100 and $200 \mathrm{~km}$ and about the same size as the mean outside of $200 \mathrm{~km}$. These asymmetries are probabiy due to the lack of enough Fourier modes to fully resolve the sharp vertical velocity peak which occurs near $60 \mathrm{~km}$. There is also some contribution from gravity wave activity which propagates through the domain and re-enters through the periodic boundary. Although not ideal, the asymmetries in the vertical velocity field are not a major problem since they do not appear to cause large asymmetries in the wind and geopotential fields.

The axisymmetry of the wind and geopotential fields at $t=96$ hours in a primitive equation model with large diabatic heat sources on a doubly-periodic domain may be somewhat surprising. The linear theory of geostrophic adjustment (e.g. Schubert et al., 1980) indicates that an impulsive diabatic heat source with a length scale less than the Rossby radius of deformation will largely excite gravity wave motion and only a small fraction of the total energy produces balanced flow. This argument is modified in regions of high inertial stability, as shown by Schubert and Hack (1982) and discussed qualitatively by Ooyama (1982). 
The large inertial stability near the center of the tropical cyclone causes the Rossby radius of deformation to become smaller, so that a larger amount of the diabatic heating will produce balanced flow. In the earlier stages of the tropical cyclone simulation, however, the model might still be expected to produce large amplitude gravity waves. The gravity waves should then propagate away from the storm, pass through the periodic boundaries and eventualiy obscure the symmetric balanced flow. The reason this does not occur is because the arguments from the linear theory are largely based on initial value problems where all the heat is added instantaneously. As discussed by Schubert et al. (1980, section 8), Hack and Schubert (1981b), and in detail by Silva Dias et al. (1983), the amount of gravity wave energy excited decreases as the time scale of the forcing becomes long compared to the period of the gravity waves. The diabatic forcing in the current model is given by the product of $\eta$ and $w$ when $w$ is positive. As can be seen in Fig. 5, $Q$ is zero initially and increases in the inner region of the vortex as the maximum in $w$ centered at about $65 \mathrm{~km}$ increases. After $\mathrm{t}=48$ hours, the maximum value of $w$ remains approximately constant, so that $Q$ decreases as $n$ decreases. Thus, a rough estimate for a characteristic period for $Q$ is on the order of four days or about 100 hours. Fig. 4a shows the periods of the gravity waves as a function of wavelength for the mid-latitude $\beta-p l$ ane. For the case when $\beta=0$, the periods are modified only slightly, since the gravity waves with the wavelengths considered here are not sensitive to the variation of the Coriol is force with latitude. For a wavelength of about $200 \mathrm{~km}$ (the approximate length scale of the w maximum in Fig. 5), the periods of the gravity waves are on the order of 1 hour for the internal mode and 0.2 hours for the 
external mode. Thus, the time scale of the diabatic forcing is much longer than the periods of the gravity waves so that much less gravity wave energy than predicted from an initial value problem should be generated.

The above argument can be verified since the governing equations are transformed so that the dependent variables are the amplitudes of the normal modes of the linear equations as discussed in section 3.3. For the incompressible fluid system, the total kinetic energy KE and available potential energy APE can be defined by

$$
\begin{aligned}
& K E=\frac{\rho}{2} \int_{0}^{L_{y}} \int_{0}^{L_{x}}\left[H_{0}\left(u_{0}^{2}+v_{0}^{2}\right)+\left(H_{1}+h_{1}\right)\left(u_{1}^{2}+v_{1}^{2}\right)+\varepsilon\left(H_{2}+h_{2}\right)\left(u_{2}^{2}+v_{2}^{2}\right)\right] d x d y \\
& A P E=\frac{\rho g}{2} \int_{0}^{L_{y} L_{x}}\left\{\left[(1-\varepsilon)\left(H_{0}+H_{1}+h_{1}\right)^{2}+\varepsilon\left(H_{0}+H_{1}+H_{2}+h_{1}+h_{2}\right)^{2}\right]-\bar{P}\right\} d x d y
\end{aligned}
$$

where

$$
\bar{P}=\left[(1-\varepsilon)\left(H_{0}+H_{1}\right)^{2}+\varepsilon\left(H_{0}+H_{1}+H_{2}\right)\right]
$$

and the layer 1 density $\rho$ is assumed to be $1.0 \mathrm{~kg} \mathrm{~m}^{-3}$. In (4.9) the available potential energy is defined as the total potential energy of the fluid minus the potential energy when $h_{1}$ and $h_{2}$ are zero. The sum of the kinetic and available potential energy was calculated using only certain modes of the solution. The basic procedure was to first set all the amplitudes $w_{j k \ell r}$ equal to zero except, for example, those corresponding to the gravity waves. The physical space variables on the transform grid defined by (3.28) were then calculated from $W_{j k \ell}$ and the integrals in (4.8) and (4.9) were evaluated using trapezodial quadrature. Similar to the calculation of the nonlinear terms using the 
transform method, the integrais are computed exactly. Using this procedure the energy of the internal rotational modes (IR), external rotational modes (ER), internal gravity waves (IG), external gravity waves (EG) and energy of the boundary layer modes (BL) were calculated for the symmetric tropical cyclone simulation.

In the linear case, the definition of kinetic energy given by (4.8) is modified by neglecting $h_{1}$ and $h_{2}$ compared to $H_{1}$ and $H_{2}$. For this case, only quadratic terms appear in the definitions of KE and APE. Assuming that the normal modes of the model are orthogonal, a Parseval relation could then be derived which related KE and APE to sums of the amplitudes of the normal modes. For this case, the sum of the modal energies described above would be equal to the total energy. In the nonlinear case, however, the cubic terms in the definition of KE must be included so that a Parseval relation can not be used to determine KE. For the nonlinear case, then, the sum of the modal energies does not add up to the total energy. In practice, however, the contribution from the cubic term in (4.8) was fairly small so that the sum of the modal energies was within a few percent of the total energy. Thus, the modal energies still give an indication of the amount of the total energy in various modes of the solution.

The energies of the modes of the model are shown in Fig. 9 as a function of time. Initially the amplitudes of the rotational modes are much larger than the gravity wave amplitudes since the mass and wind fields are in gradient wind balance. The gravity modes are not identically zero, however, since the modal decomposition is based on linear theory while the gradient wind equation (nonlinear balance equation) contains a nonlinear term. The amplitudes of the boundary 


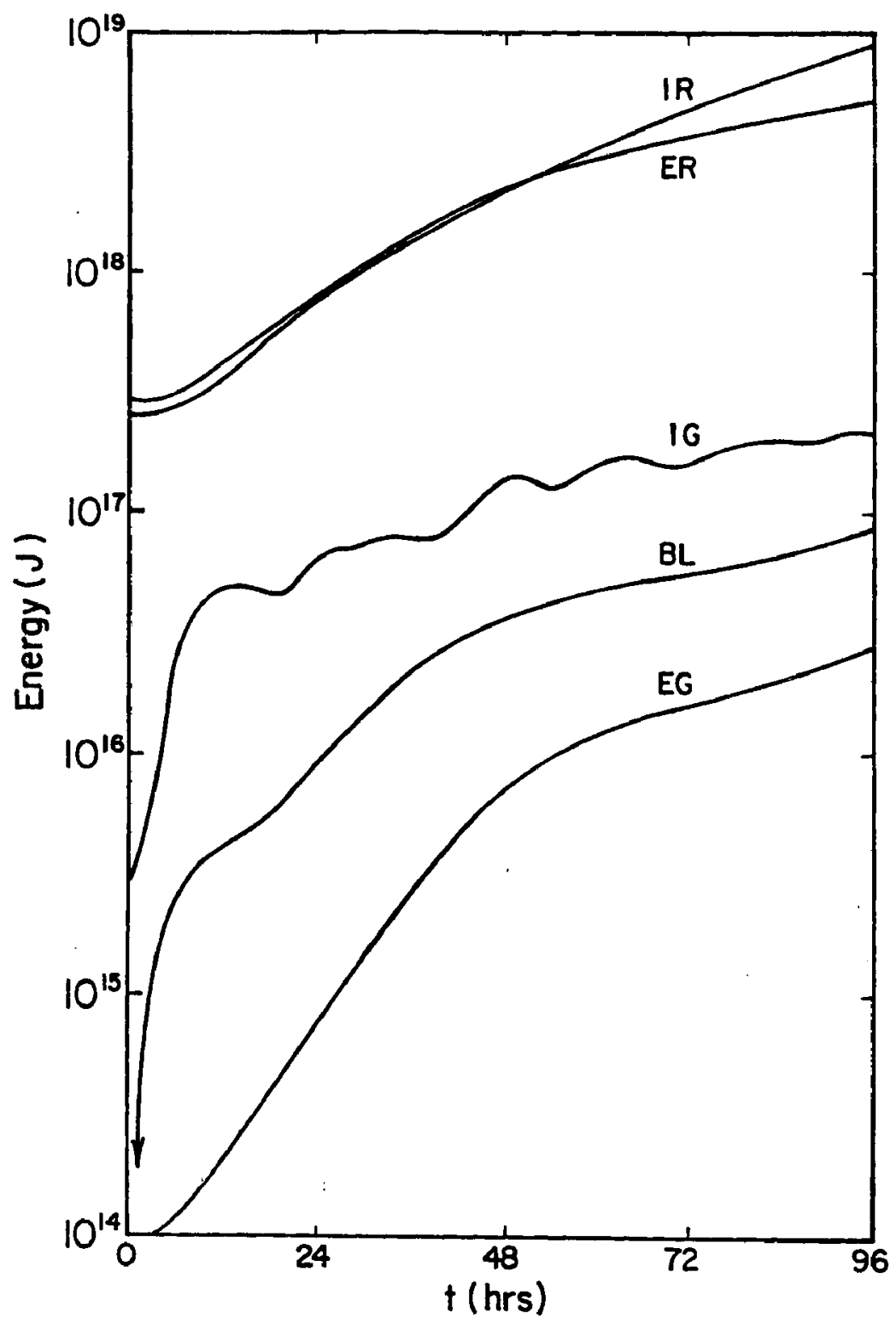

Figure 9. The time evolution of the energy in the internal rotational modes (IR), external rotational modes (ER), internal gravity modes (IG), external gravity modes (EG). and the energy in the boundary layer modes (BL) for experiment $4 A$. 
layer modes are identically zero initially since it was assumed that the layer 0 and layer 1 wind fields were the same at $t=0$. As time increases, the energy of all the modes increases, with the external gravity waves and boundary layer modes increasing the most rapidly initially. After about 12 hours, the energy of the internal gravity waves and boundary layer modes increase at a slower rate as the system appears to have adjusted to initial development of the boundary layer vertical velocity.

The most important feature which can be seen in Fig. 9 is that the energy of the rotational modes is much larger than the energy of the gravity modes. By 96 hours the energy in either the external or internal rotational modes is more than an order of magnitude larger than the energy in the internal gravity waves and two orders of magnitude larger than the external gravity wave energy. This indicates that the time scale of the forcing modifies the arguments of the linear theory of geostrophic adjustment and also that the use of periodic boundary conditions in the current model is not as severe an approximation as might be thought.

The fact that the energy in the gravity modes is more than an order of magnitude less than the energy in the rotational modes in Fig. 9 has some implications for primitive equation tropical cyclone models which include more general boundary conditions. Hack and Schubert (1981b) have developed a boundary condition for a grid-point model which minimizes the reflection of gravity waves. Their approach, which is based on the work of Bennett (1976), considers linearized versions of the primitive equations in cyclindrical coordinates on an f-plane. In order to develop a boundary condition which can be used in practice, it 
is necessary to consider limiting cases of the linearized equations which give radiation conditions for pure gravity waves or a condition appropriate for the balanced flow. Hack and Schubert investigate the radiation condition for the gravity waves, but the results presented here indicate that at times, the boundary condition for the balanced flow may be more appropriate. The correct choice probably depends on the length and time scales of the diabatic forcing in a particular model. For example, the gravity wave radiation condition might be appropriate for tropical cyclone models with explicit release of latent heat (e.g. Rosenthal, 1978) since the diabatic forcing varies on shorter time scales than in the current model, while the balanced flow condition might be appropriate for models with cumulus parameterization schemes with slowly varying diabatic forcing.

In primitive equation tropical cyclone models which use explicit time differencing schemes, the time step is limited by the speed of the external gravity waves. This restriction can be removed through the use of semi-implicit methods (Kwizak and Robert, 1971) at the expense of phase errors in the external gravity waves. As shown in Fig. 9, the energy in the external gravity wave part of the solution is more than two orders of magnitude smaller than the energy of the rotational mode part of the solution. This indicates that the use of semi-implicit methods in primitive equation tropical cyclone models is probably justifiable.

As discussed in chapter 2, diabatic heating in the incompressible fluid system is represented by a mass transport from layer 1 to layer 2. During a model integration, the mean depth of layer 1 decreases and the mean depth of layer 2 increases, which causes the mean potential energy 
in the domain to increase with time. The model is initialized so that the mean depth of layers 1 and 2 are given by $H_{1}$ and $H_{2}$. As the model is integrated in time, the $k=l=0$ modes of $h_{1}$ and $h_{2}$ increase as the diabatic forcing becomes active. Since the $k=\ell=0$ mode of a double Fourier series represents a horizontal average, these modes of $h_{1}$ and $h_{2}$ give some idea of how the mean potential energy of the domain increases with time. The available potential energy defined by (4.9) was then computed using only the $k=\ell=0$ modes of $h_{1}$ and $h_{2}$ at various times as shown in Fig. 10 (curve M). Also shown in Fig. 10 are the total energy of the domain $(T)$ and the sum of the energy in the external and internal rotational modes from Fig. 9. Fig. 10 shows that the largest contribution to the increase in the total energy in the domain comes from the increase in the mean potential energy. The fact that the amount of energy in the rotational modes is much smaller than the total energy in Fig. 10 indicates that the diabatic heat source is inefficient at producing balanced flow, in agreement with the linear theory. The diabatic heat source does not, however, produce large amplitude gravity waves, but rather increases the mean potential energy in the domain. The change in the mean depths of layers 1 and 2 during a model integration has only a small effect on the dynamics of the large scale environment (the phase speeds of the internal gravity waves are altered slightly). The change in the mean depth of layer 2 does, however, have an effect on the diabatic heat source since it affects the diagnosed value of $\Omega_{2}$. This effect was discussed previously and is analogous to a net heating of the model domain. In future versions of the model, a crude radiation parameterization could be included by allowing mass to be transported from layer 2 back to layer 1 to crudely simulate the 


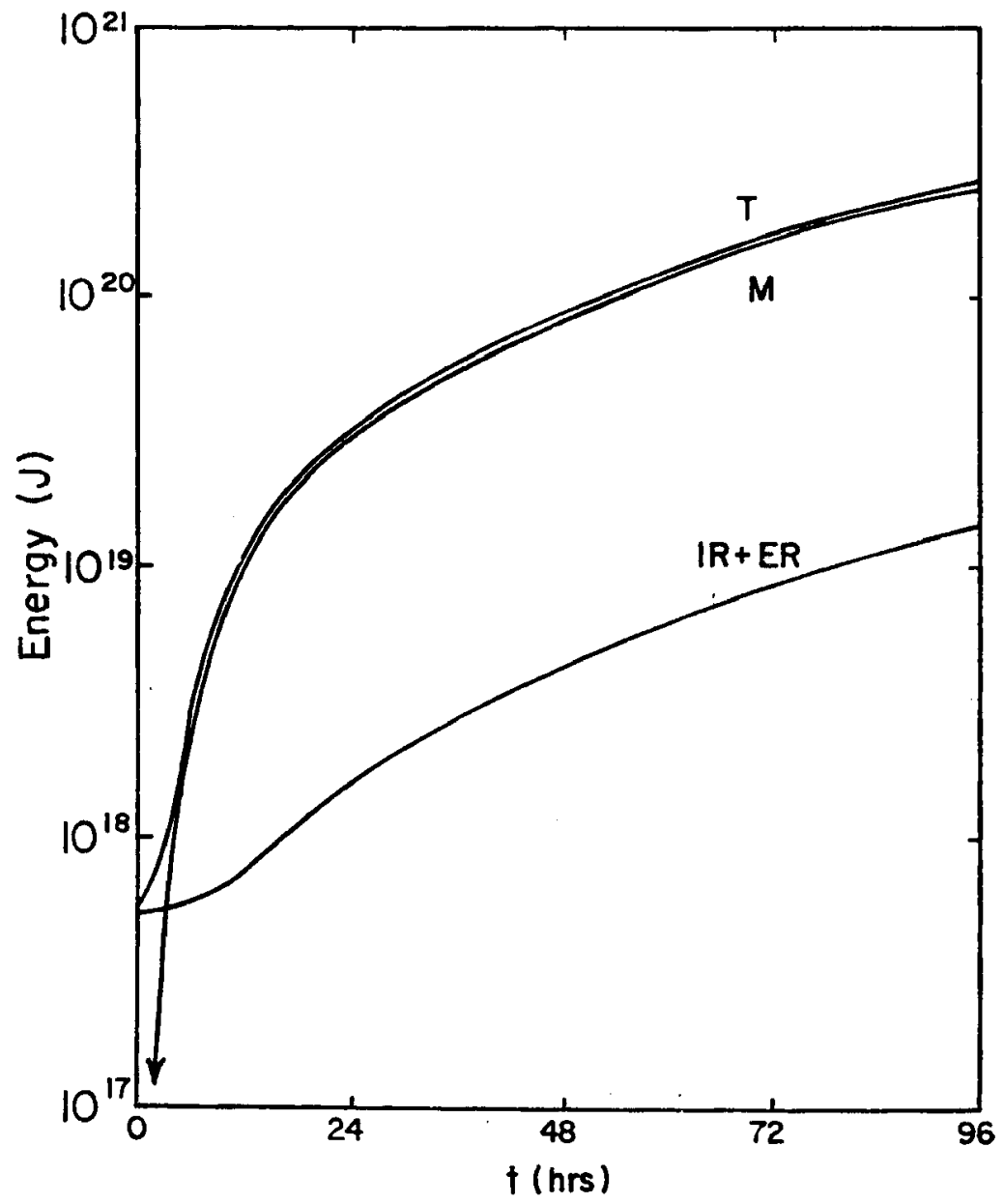

Figure 10. The time evolution of the total energy in the domain $(T)$, the energy of the $k=\ell=0$ mode $(M)$ and the sum of the internal and external rotational mode energies (IR+ER) for experiment $4 \mathrm{~A}$. 
radiative cooling of the atmosphere. This would allow for longer model simulations when the diabatic heat source was active.

\subsection{The Effect of a Variable Coriol is Parameter on a Tropical Cyclone Simulation}

In order to investigate the effect of a variable Coriol is parameter on a tropical cyclone simulation, the model was run with the initial condition described in the previous section, with $\beta$ evaluated at $20^{\circ} \mathrm{N}$. The initial vortex is confined to layers 0 and 1 and has a maximum tangential wind of $10 \mathrm{~ms}^{-1}$ at a radius of $100 \mathrm{~km}$, as in experiment $4 \mathrm{~A}$. As described in section 2.4, it was necessary to neglect the streamfunction contribution to the $\beta$ term in the divergence equation (2.33) in the current model formulation. For this reason, there is no $\beta$ term in the nonlinear balance equation (4.4) which was used to obtain the initial mass field from the wind field. Thus, the initial wind and mass fields for the case when $\beta$ is included are axisymmetric, so that the initial conditions in experiments $4 A$ and $4 B$ are identical.

One of the first studies which considered the effect of a variable Coriolis parameter on a tropical cyclone was presented by Rossby (1948). Rossby concluded that the larger value of the Coriolis parameter on the poleward side of an axisymmetric cyclonic vortex would cause a poleward acceleration of the vortex. More recent studies (e.g. Anthes and Hoke, 1975; Kitade, 1980) indicate that Rossby's argument must be modified since an initially axisymmetric vortex will become asymmetric as it begins to move. The later results indicate that for barotropic and baroclinic models, a vortex similar to a tropical cyclone will move towards the northwest at speeds between 1 and $3 \mathrm{~ms}^{-1}$. The current model 
is in agreement with these results as can be seen in Fig. 11 which shows the track of the streamfunction minimum for experiment 4B. Fig. 11 also shows the speed and direction of the tropical cyclone as a function of time which were calculated using three-hourly positions of the streamfunction minimum. During the simulation, the model tropical cyclone accelerates until about 60 hours after which time the storm maintains a constant speed of about $2.5 \mathrm{~ms}^{-1}$. The motion is towards a direction slightly north of northwest with some indication of a more northwesterly motion after about 84 hours. The cyclone track shown in Fig. 11 is quite similar to results presented by Jones (1977a). He found that when a variable Coriol is parameter is included in a numerical tropical cyclone simulation, the vortex moves north-northwestward in the developing stage and northwestward in the mature stage.

The tropical cyclone in experiment $4 B$ differed from the tropical cyclone in experiment $4 A$ in several ways, in addition to the storm movement. Fig. 12 shows the minimum surface pressure deviation $P_{S}$ (defined by 4.7 ) and the maximum layer 1 tangential wind VT1 for each of these simulations. From Fig. 12 it can be seen that the intensification rate for each experiment is very similar until about 48 hours. After this time, the intensity of the storm on the $\beta$-plane begins to level off, while the storm on the f-plane continues to intensify. By 96 hours, the f-plane storm is about $15 \mathrm{mb}$ deeper with maximum winds about $10 \mathrm{~ms}^{-1}$ stronger than the $\beta-p l$ ane storm. Thus, the inclusion of a variable Coriolis parameter appears to inhibit the intensification of the tropical cyclone. This result is somewhat similar to results presented by Madala and Piacsek (1975). They have shown that in a 

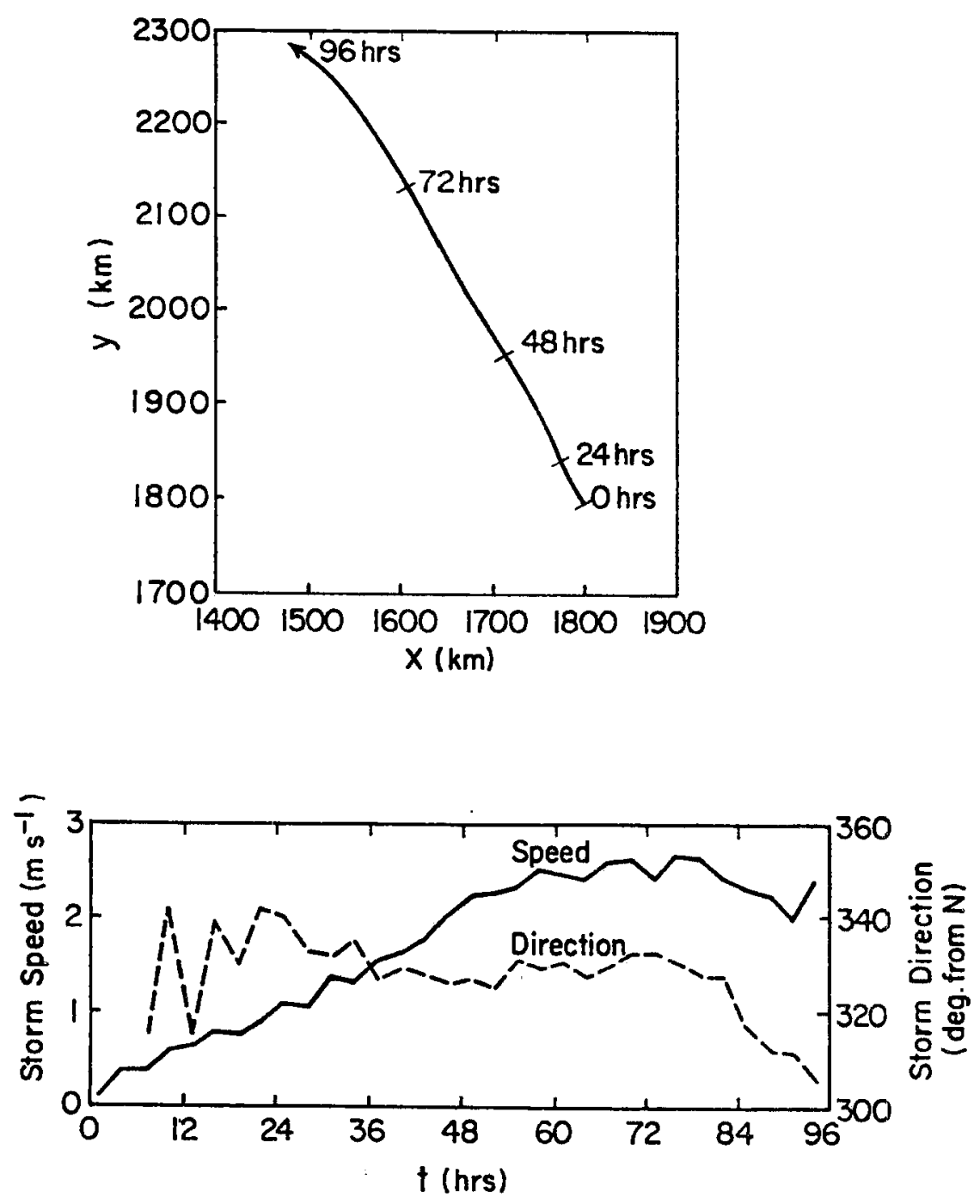

Figure 11. The track of the streamfunction minimum for the tropical cyclone in experiment $4 \mathrm{~B}$ (upper) and the time evolution of the speed and direction of motion (lower). 

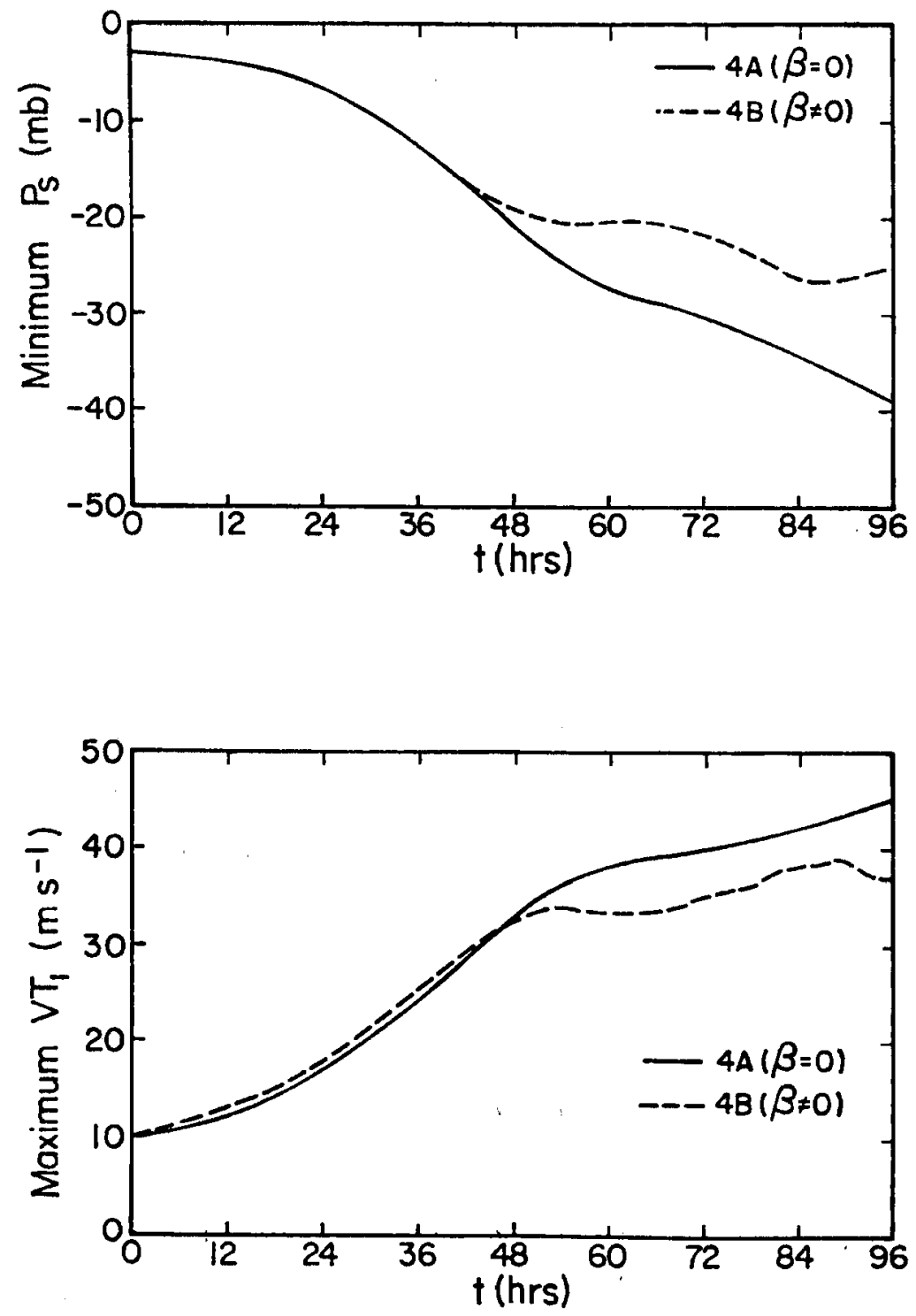

Figure 12. The time evolution of the minimum surface pressure deviation (upper) and the maximum layer 1 wind speed (lower) for experiments $4 A$ and $4 B$. 
three-layer tropical cyclone model, a storm took longer to reach hurricane intensity when a variable Coriol is parameter was included.

In order to further compare experiments $4 \mathrm{~A}$ and $4 \mathrm{~B}$, the azimuthally averaged values of several of the dependent variables were calculated from a cylindrical grid similar to that described for experiment $4 \mathrm{~A}$. For experiment 4B, however, the cylindrical coordinate system was always centered on the streamfunction minimum as the storm moved. Fig. 13b shows the azimuthally averaged layer 1 and 2 tangential winds, layer 1 and 2 geopotentials, boundary layer vertical velocity and convective stability parameter for experiment $4 B$ at 96 hours. The same variables are also shown for experiment $4 \mathrm{~A}$ at 96 hours (repeated from Fig. 5) in Fig. 13a for comparison. Comparing Fig. 13a with 13b it can be seen that the radial structure of the layer 1 variables is quite similar for each experiment, except that the vortex on the $f-p l a n e$ is somewhat more intense. The convective stability parameter $n$ and $w$ are also very similar for each experiment. The major difference between the two simulations is the structure of the upper layer tangential wind profile and corresponding geopotential. For the $f-p l a n e$ case there is a cyclonic vortex in the upper layer inside about $300 \mathrm{~km}$ which has a maximum tangential wind almost as large as for layer 1. For the $\beta-p l a n e$ case there is also a cyclonic vortex in layer 2 inside about $300 \mathrm{~km}$, but the tangential wind speeds are much smaller than those of layer 1 . This difference in structure is a result of the transport of momentum from layer 1 to layer 2 by the diabatic term Q. As described in section 2.2, when fluid is transported from layer 1 to layer 2 it conserves the momentum of layer 1 as it mixes with the fluid in layer 2. The cyclonic vortex which forms in layer 2 for the $f-p l a n e$ case is a result of this 

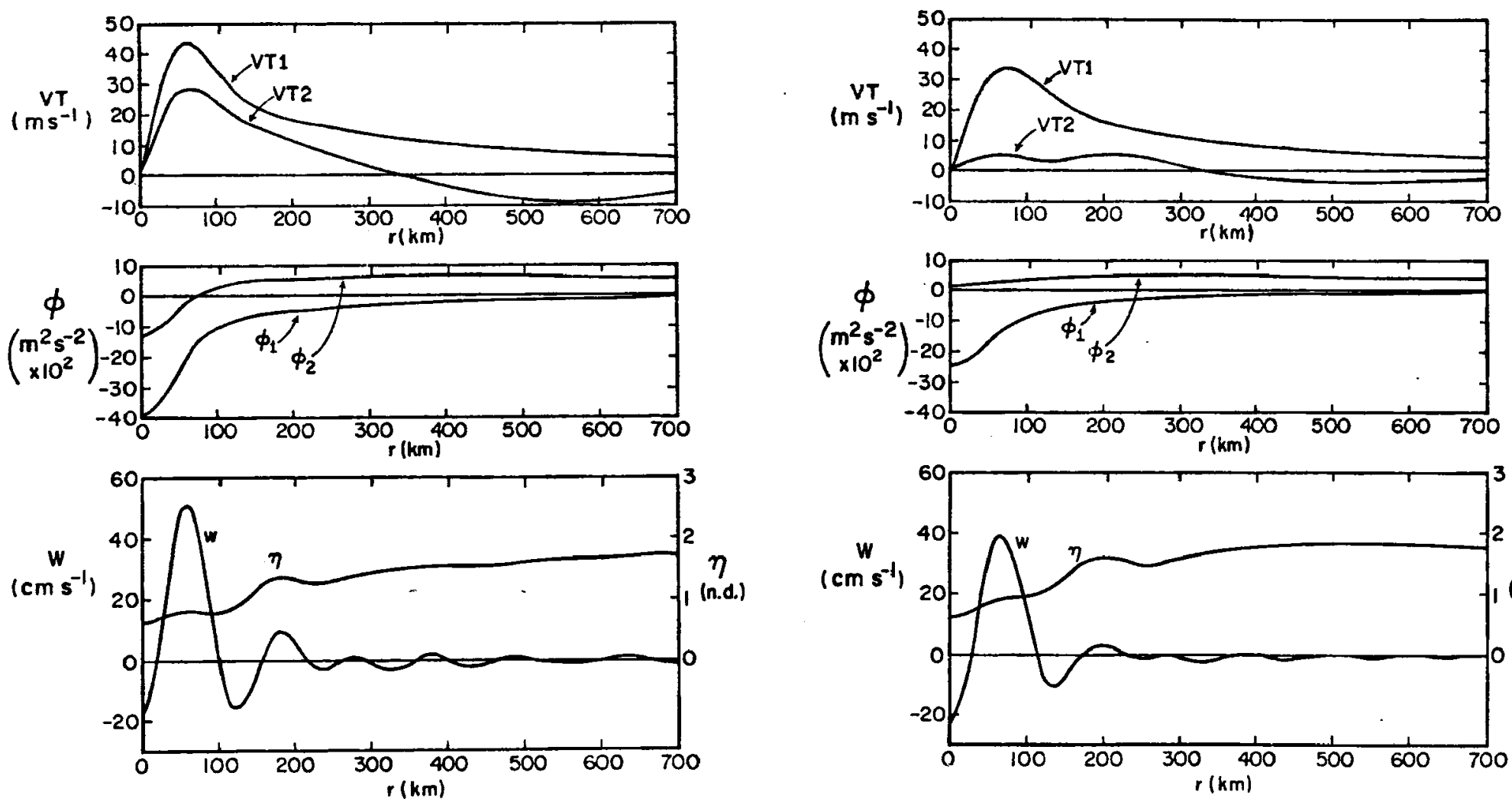

(a)

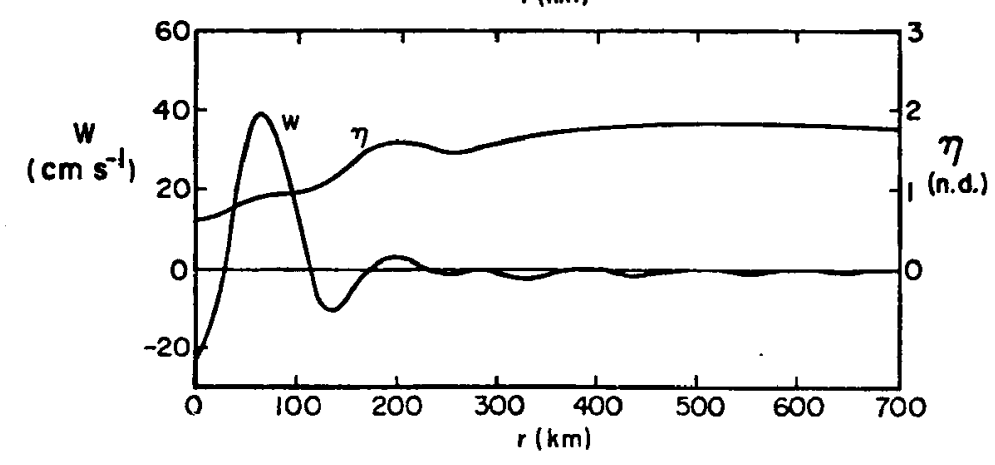

Figure 13. The azimuthally averaged tangential wind VT and geopotential $\phi$ for 1 ayer 1 and 2 , vertical velocity at the top of the boundary layer $w$ and vertical stability parameter $n$ at 96 hours for experiments $4 A(a)$ and $4 B(b)$. 
process. This was verified by repeating the f-plane experiment with the transport term set to zero. For the $\beta-p l a n e$ case, the tropical cyclone begins to move due to the differential advection of the earth's vorticity by the storm circulation. When a vortex forms in the upper layer of the model, it is also begins to move by this process. Since the radial structure of the upper and lower layer vorticies are different, the motion induced by the B-effect will also be different. Thus, it is more difficult to establish a concentrated area of cyclonic rotation in the upper layer by momentum transport for the $\beta$-plane case since the center of the upper layer vortex does not always remain directly over the lower layer vortex. Vertical wind shear should also affect the vortex in a similar manner since this would also cause the motion of the upper and lower layer vorticies to be different. Assuming that cumulus clouds in nature also transport momentum in the vertical, the cyclonic vortex for tropical cyclones in low vertical wind shear environments should extend to higher altitudes than for storms in environments with large vertical wind shear.

The difference in the structure of the upper layer tangential winds in the f-plane and $B-p l a n e$ simulations may help explain the difference in the intensification rates. In Fig. 13, the layer 2 tangential wind for the f-plane storm has anticyclonic shear between about $80 \mathrm{~km}$ and 550 $\mathrm{km}$, while the shear for the $B-p l a n e$ storm is much weaker. For the $f$ plane storm, the layer 2 shear vorticity is larger than the curvature vorticity outside of about $100 \mathrm{~km}$ so that the relative vorticity is negative outside this radius. This indicates that the inertial stability will be lower in this region for the f-plane storm than for the $\beta-p l a n e$ storm since the inertial stability is related to the 
absolute vorticity. This indicates that it may be easier for the fplane storm to establish a radial circulation in the upper layer since the inertial stability is a measure of the resistance to horizontal motion. The enhanced radial circulation for the f-plane storm would allow mass to be removed from the inner regions and allow the surface pressure to decrease which would intensify the storm.

Another difference between experiments $4 A$ and $4 B$ which can be seen in Fig. 13 is that the layer 2 tangential wind outside a radius of about $300 \mathrm{~km}$ has a smaller magnitude for the $\beta-p l$ ane case. Since the $\beta-p l a n e$ storm is weaker than the $f$-plane storm, it might be expected that the $\beta$ plane storm would also have a weaker anticyclone. Another reason for this difference is that the outflow for the $\beta$-plane case is highly asymmetric, so that only a portion of the wind field is represented by the azimuthally averaged tangential wind. Fig. 14 shows the twodimensional structure of the wind and geopotential fields of layers 1 and 2 for the $\beta-p l a n e$ simulation at 96 hours. In Fig. 14, only a portion of the domain is shown, and the fields which are contoured are the geopotential heights $(\phi / g)$ which have dimensions of length. The contour interval is $20 \mathrm{~m}$ for layer 1 and $10 \mathrm{~m}$ for layer 2. As shown in (4.7), the layer 1 geopotential can be interpreted in terms of a surface pressure deviation. In Fig. 14, a contour interval of $20 \mathrm{~m}$ approximately corresponds to a surface pressure contour interval of 2 mb. In Fig. 14 the asymmetric structure of the wind and geopotential fields for layer 2 can be seen. There is some evidence of a cyclonic vortex near the storm center which is axisymmetric with respect to the storm center. Outside the cylconic region, however, the flow is highly asymmetric with evidence of an outflow channel towards the south and 

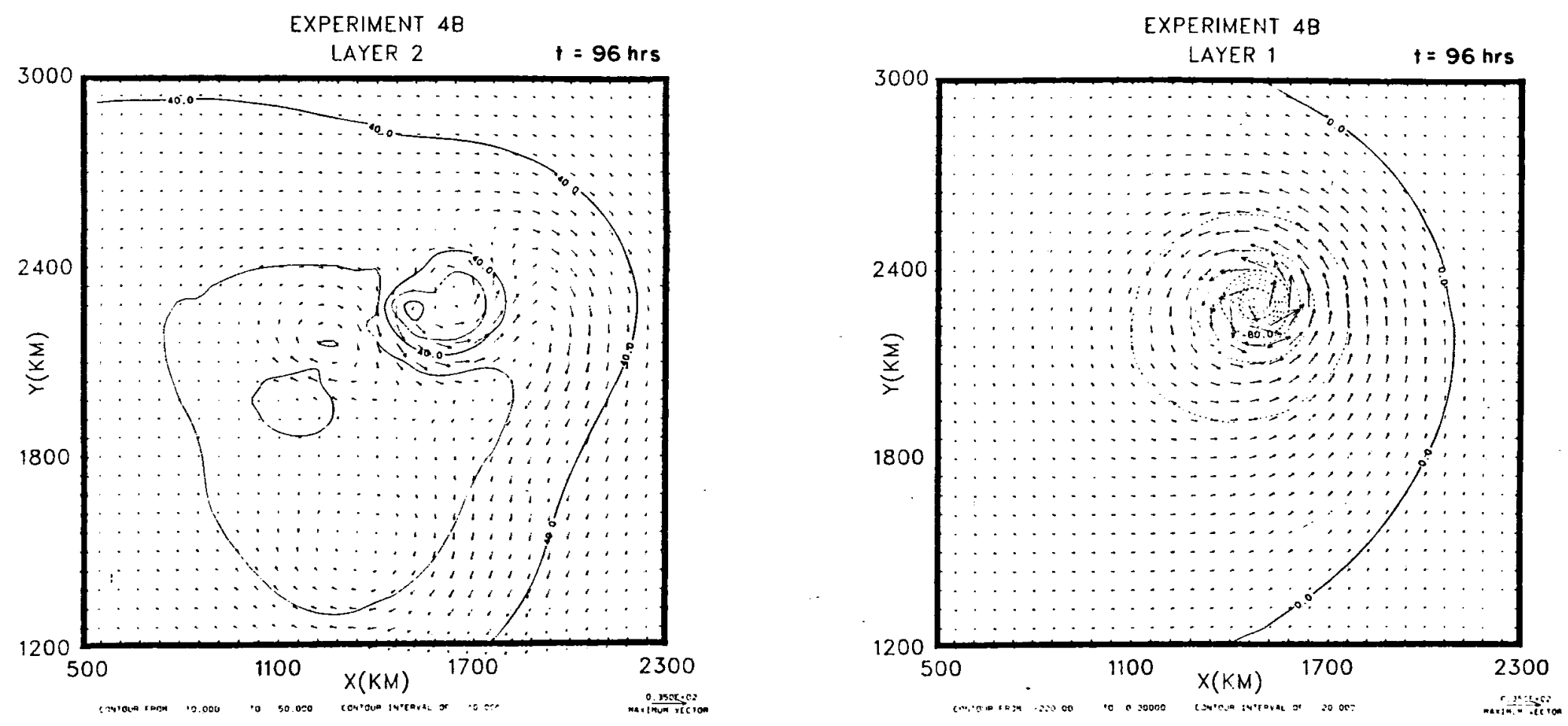

Figure 14. The layer $i$ and 2 horizontal wind and geopotential height fieids for experiment $4 B$ at 96 hours. The height contour interval is 20 and $10 \mathrm{~m}$ for layers 1 and 2 respectively. 
southwest which begins on the eastern part of the storm. There is also some evidence of a small, closed anticyclone to the west of the storm center in layer 2 . This rather complicated pattern is probably a result of the $\beta$-effect and also the momentum transport from layer 1 to layer 2. The structure of the wind field in the upper layer is similar to some storms which have been observed. Chen (1983) has presented a study of the upper level flow patterns associated with tropical cyclones in several ocean basins using 12 months of FGGE data. Observations from 79 cases where divided into three groups consisting of storms with one outflow channel (57 cases), two outflow channels ( 5 cases) and no outflow channel (17 cases). Of the storms with single outflow channels, 28 cases were towards the pole while 29 cases where towards the equator similar to the simulation in experiment $4 \mathrm{~B}$. Thus, the structure of the upper layer in the model simulation is similar to about $37 \%$ of the cases presented by Chen. The outflow from many of the storms in the study by Chen was affected by interaction with mid-latitude westerlies. It is interesting to note that in Chen's study, 8 out of 12 cases from storms in the N.E. Pacific had single outflow channels towards the equator. Since these storms generally form close to the equator (Gray, 1979) they are more likely to be far removed from the westerlies, and more representative of experiment $4 B$, which had no basic state wind.

In Fig. 14 it can be seen that the layer 1 fields are much more axisymmetric than the layer 2 fields, although some asymmetry exists. The vortex is elongated towards the west at large radii, with some indication of a sharper geopotential gradient towards the east at small radii. This structure can be explained by considering the dispersive properties of the Rossby waves. As can be seen from (3.73) for the 
linear case $\left(P_{j k \ell r}=0\right)$, the analytic solution for each of the model normal modes is proportional to $e^{i v t}$ where $\nu$ is the frequency of a given mode. For this case the phase speed is given by $-(\nu / k)$ so that a positive frequency corresponds to a westward propagating wave. Similarly, the zonal component of the group velocity for this case is given by $-\frac{\partial \nu}{\partial K}$. The phase speed determines the speed of propagation of the individual waves while the group velocity determines the propagation of energy by a group of waves. Fig. $4 b$ shows the frequencies of the Rossby waves in the model. In Fig. 4b, the negative of the slope of the frequency curves determines the zonal component of the group velocity, so that the group velocity is negative for low zonal wave numbers and positive for high zonal wavenumbers. This indicates that the long Rossby waves disperse energy towards the west, while the short Rossby waves disperse energy towards the east. Thus, the elongation of the vortex to the west at large radi $i$ is due to the dispersion of the long Rossby waves.

In Fig. 14, the sharper geopotential gradients in layer 1 are to the east of the storm center, although they are to the west of the initial position of the storm. Thus, the eastward dispersion of the short Rossby wave part of the solution does not appear to explain this feature. The nonlinear interaction of the symmetric vortex and the advection of the earth's vorticity have resulted in a mean flow which causes the vortex to move towards the northwest. Thus, the sharper geopotential gradients may be interpreted in terms of the eastward dispersion of the short Rossby wave part of the solution where the entire pattern is advected by a mean flow. 
The asymmetric structure of the layer 1 variables is also similar to the structure of storms observed in nature. Fig. 15 shows streamlines and isotachs at the $850 \mathrm{mb}$ level from a composite of 46 developing hurricanes in the Australian region from Holland (1983). Although the value of the Coriolis parameter is negative in the southern hemisphere, the value of $B$ is positive so that the frequencies of the Rossby waves are the same as for the northern hemisphere. In Fig. 15 it can be seen that the composite hurricane is elongated towards the west at large radii (about $10^{\circ}$ longitude from the center) with the higher wind speeds towards the east at smaller radii $\left(2-5^{\circ}\right.$ longitude from the center). Thus, the asymmetric structure of the lower levels of the composite hurricane can be explained by considering the dispersion of energy by the Rossby waves, similar to that of the lower layer of the model tropical cyclone shown in Fig. 14.

In experiment $4 \mathrm{~B}$, asymmetries also developed in the dependent variables which are not shown in Fig. 14. In particular, the boundary layer vertical velocity was quite asymmetric at very small radii (less than $200 \mathrm{~km})$. These asymmetries appear to be related to the motion of the storm, rather than to the dispersion of wave energy. These features will be discussed in greater detail in the next section when results from an experiment with a basic state wind are presented.

\subsection{Simulation of A Tropical Cyclone With A Non-Resting Basic State}

In the real atmosphere, the occurrence of an isolated symmetric tropical cyclone is quite rare. More commonly, storms are imbedded in some type of mean current which interacts with the storm circulation. 


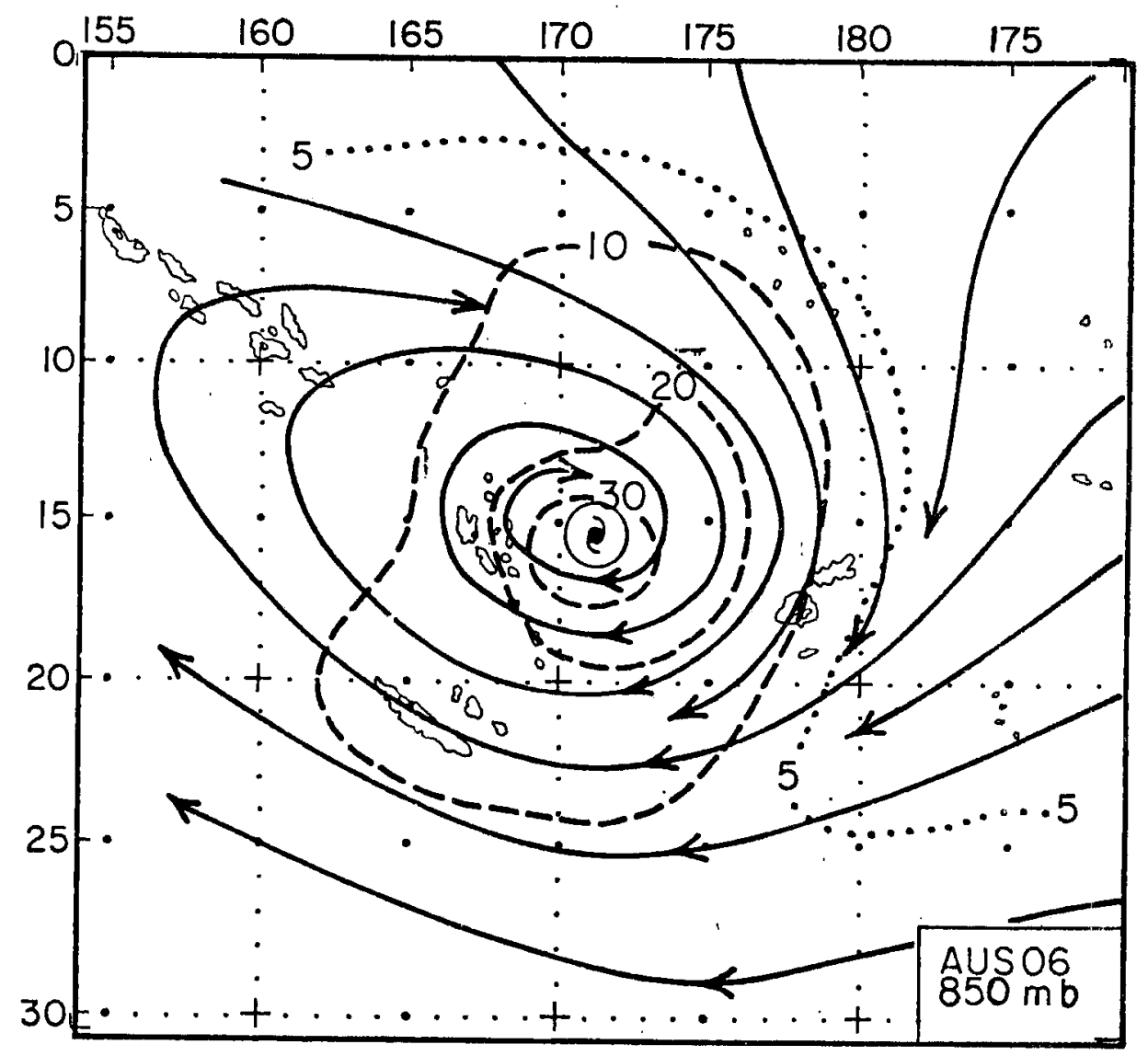

Figure 15. Streamlines and isotachs at the $850 \mathrm{mb}$ level from a composite of 46 developing hurricanes in the Australian region (from Holland, 1983). 
Experiment $4 C$ is an example of a tropical cyclone simulation which includes a zonal wind.

When periodic boundary conditions are used in the north-south direction the form of the zonal wind which can be included is somewhat restricted. For example, it is not possible to use a zonal wind which is constant everywhere in the domain. This can not be used since it would be necessary to include a height field which was linear in $y$ (neglecting $\beta$ ) in order to satisfy geostrophic balance, which would not satisfy the periodic boundary condition. Thus, it is necessary to specify the zonal wind in such a way that it is periodic and has a periodic anti-derivative. An obvious choice for a set of functions which satisfy these conditions are trigonometric functions. Another reason for choosing trigonometric functions is that it is trivial to determine the Fourier series representations, which contain onty one term. In experiment $4 C$, the zonal wind for layer $i$ was specified to be

$$
\bar{u}_{i}=U_{i} \sin \left(\frac{2 \pi y}{L_{y}}\right)
$$

where $U_{i}$ was chosen to be $-7.5 \mathrm{~ms}^{-1}$ for each layer. The zonal wind given by (4.10) corresponds to a single sine wave in the north-south direction, with easterlies in the southern half of the domain and westerlies in the northern half of the domain.

In experiment $4 C$, the vortex defined by (4.1) was added to the zonal wind in layers 0 and 1 , and the mass field was determined using the nonlinear balance equation as described in section 4.1. The initial vortex for this experiment differs from the one used in experiments $4 \mathrm{~A}$ and $4 B$ in that the radius of maximum wind was $150 \mathrm{~km}$, and the vortex was centered at $x=2200 \mathrm{~km}$ and $y=1400 \mathrm{~km}$ on a 3600 by $3600 \mathrm{~km}$ domain. A 
larger initial vortex was used to allow better resolution of the asymmetries which are induced by the motion of the storm. The initial position of the vortex was chosen to crudely simulate storms which form in an easterly current and eventually recurve due to the $\beta$-effect and the influence of mid-latitude westerlies. For experiment $4 C$, the remaining parameters and variables are the same as those which were used for experiments $4 \mathrm{~A}$ and $4 \mathrm{~B}$.

The upper portion of Fig. 16 shows the track of the tropical cyclone in experiment $4 C$. In this figure it can be seen that the storm tracks westward initially but turns towards the north, and eventually towards the northeast after 96 hours. This track appears to be a result of the steering current and the $\beta$-effect. The initial motion of the storm is simply a result of the advection by the easterly current. After a short time, the $\beta$-effect adds a northward component to the direction of motion, which carries the storm out of the easterly current and into the westerly current. This accounts for the turn towards the northeast near the end of the 5 day integration.

Fig. 16 also shows the minimum surface pressure deviation and the maximum layer 1 wind speed for experiment 4C. For this case, the initial value of the layer 1 wind speed is greater than $10 \mathrm{~ms}^{-1}$ since the initial vortex and basic state wind were added together. From this figure it can be seen that most of the development takes place during the first 60 hours of the integration. After this time, the storm maintains a relatively constant intensity equivalent to a minimal hurricane. Comparing Fig. 16 with Fig. 12 it can be seen that the intensification which occurs in experiment $4 C$ is similar to that in experiment $4 B$, but differs from experiment $4 A$ which produced a much more 

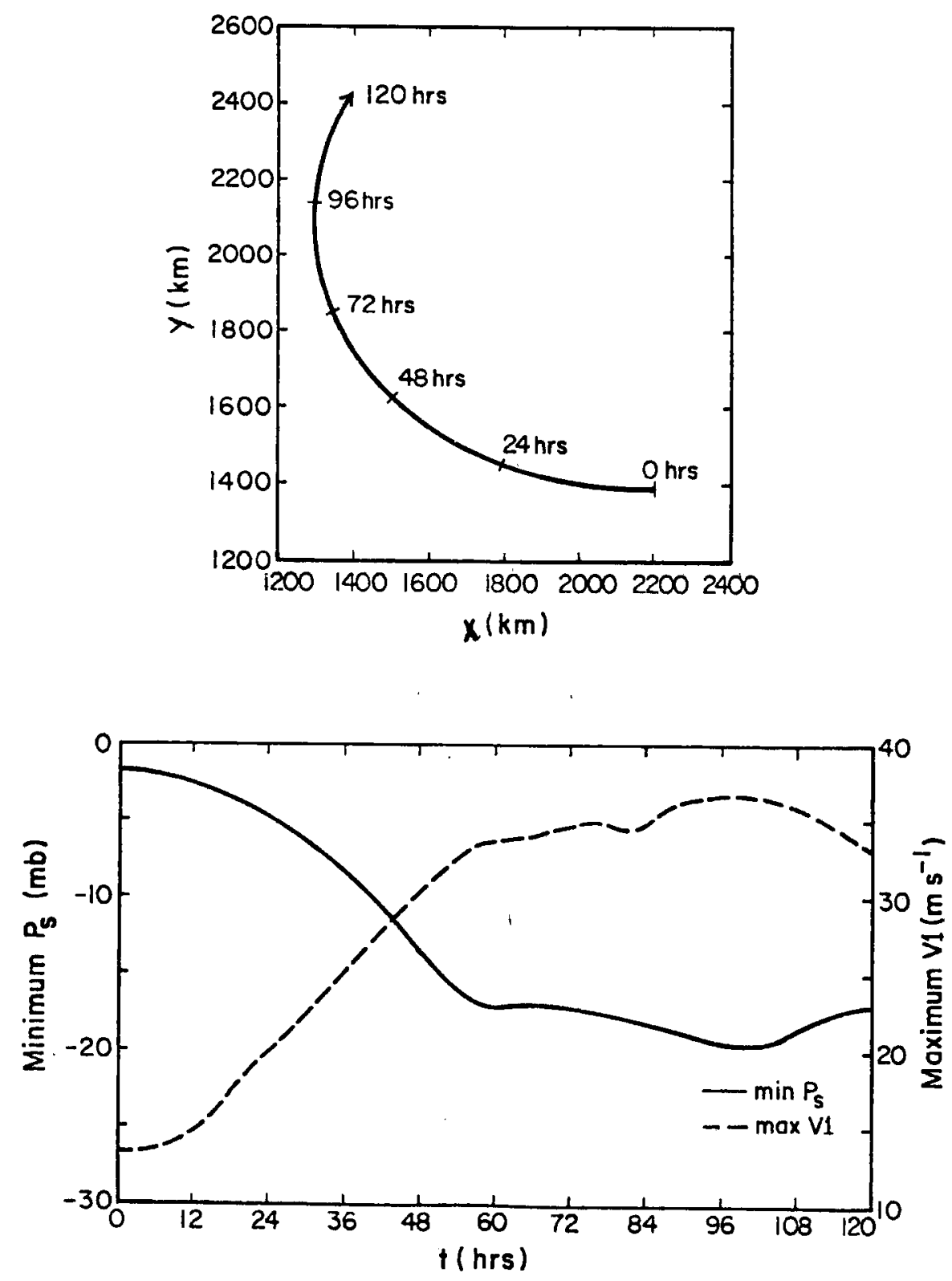

Figure 16. The track of the streamfunction minimum (upper) and the time evolution of the minimum surface pressure deviation and maximum layer 1 wind speed for the tropical cyclone in experiment $4 \mathrm{C}$ (1ower). 
intense storm. This difference appears to be related to the motion of the storm, similar to that discussed for experiment $4 \mathrm{~B}$.

In order to see the three-dimensional structure of experiment $4 C$, the wind and geopotential fields for layers 0,1 and 2 are shown in Fig. 17 at $t=96$ hours. Similar to Fig. 14 , only a portion of the domain is shown and the geopotential height contours are $20 \mathrm{~m}$ and $10 \mathrm{~m}$ for layers 1 and 2 respectively. For layer 0 , the surface pressure deviation is shown with a contour interval of $2 \mathrm{mb}$. In layers 0 and 1 , the asymmetries of the wind and height fields at large radii are similar to those presented for experiment 4B. The vortex appears to be somewhat elongated towards the west which is probably caused by the westward dispersion of the long Rossby wave part of the solution. Close to the center, however, the vortex appears to be quite symmetric, despite the presence of a basic state wind. There does appear to be some tendency for confluence to the east of the storm, which is most evident in layer 0 . As will be discussed when the boundary layer vertical velocity is shown, this is probably caused by both the $\beta$-effect and the motion of the storm. The structure of the layer 2 wind and geopotential fields are considerably more asymmetric than the lower layer fields. Similar to experiment $4 B$, there is a cyclonic vortex in the upper layer near the horizontal position of the lower layer vortex which results from the upward transport of momentum by the diabatic heat source. Fig. 17 also shows that there is some tendency for the outflow in layer 2 to be towards the south and on the eastern side of the storm as was the case in experiment 4B. Although the tropical cyclone in the lower layers affects the layer 2 flow field, it appears that layers 1 and 2 do not always remain coupled. In Fig. 17, the cyclonic vortex in layer 2 which 

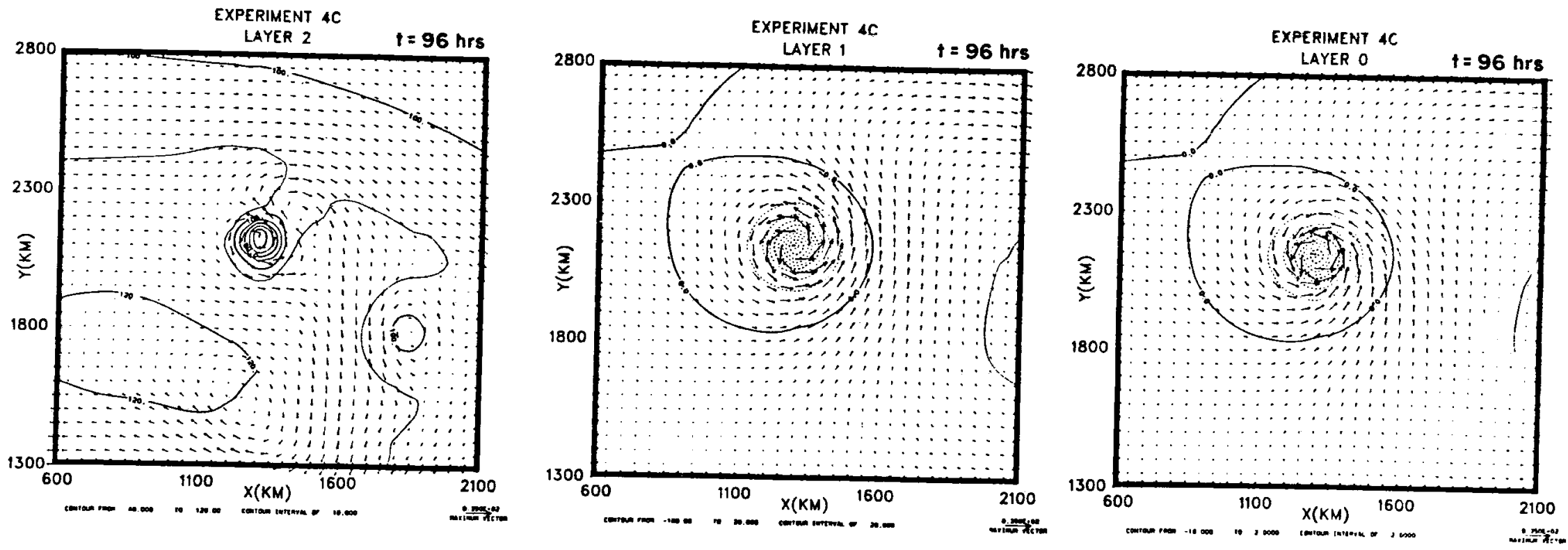

Figure 17. The wind and geopotential height fields for layers

1 and 2 and the wind and surface pressure deviation fields for layer 0 for experiment $4 C$ at 96 hours. The height contour intervals for layers 1 and 2 are 20 and $10 \mathrm{~m}$ and the pressure contour interval for layer 0 is $2 \mathrm{mb}$. 
is about $500 \mathrm{~km}$ to the southeast of the tropical cyclone center and the anticyclonic region about $750 \mathrm{~km}$ to the south-southwest are probably a result of the interaction of the outflow from the storm and the basic state wind, but appear to be left behind as the storm moves.

In addition to the horizontal wind and mass fields, the boundary layer vertical velocity and equivalent potential temperature deviation also became quite asymmetric in experiment 4C. Fig. 18 shows the boundary layer vertical velocity field in the region surrounding the tropical cyclone at $24,48,72$ and 96 hours. Initially the vertical velocity is zero since the initial vortex is nondivergent. By 24 hours, a vertical velocity field has developed with a maximum of about $20 \mathrm{cms}^{-1}$ located in the right front part of the storm in relation to the direction of motion (the arrow indicates the direction of motion of the storm). There is also a broad area of rising motion on the eastern side of the storm which spirals back towards the southwest. At 48 hours, the vertical motion pattern is similar to that at 24 hours although the vertical motion maximum in the right front part of the storm has increased to over $40 \mathrm{cms}^{-1}$. This pattern continues at later times as the storm moves towards the north, and then towards the northeast, with the vertical motion maximum remaining in the front and slightly to the right side of the storm. The larger area of vertical motion on the east side of the storm also persists, although it is becoming less well defined by 72 hours.

The spiral zone of rising motion to the east of the tropical cyclone in Fig. 18 appears to be related to the $\beta$-effect. This is similar to the result presented by Anthes and Hoke (1975), who showed that the inclusion of $\beta$ in a barotropic model causes a spiral shaped 

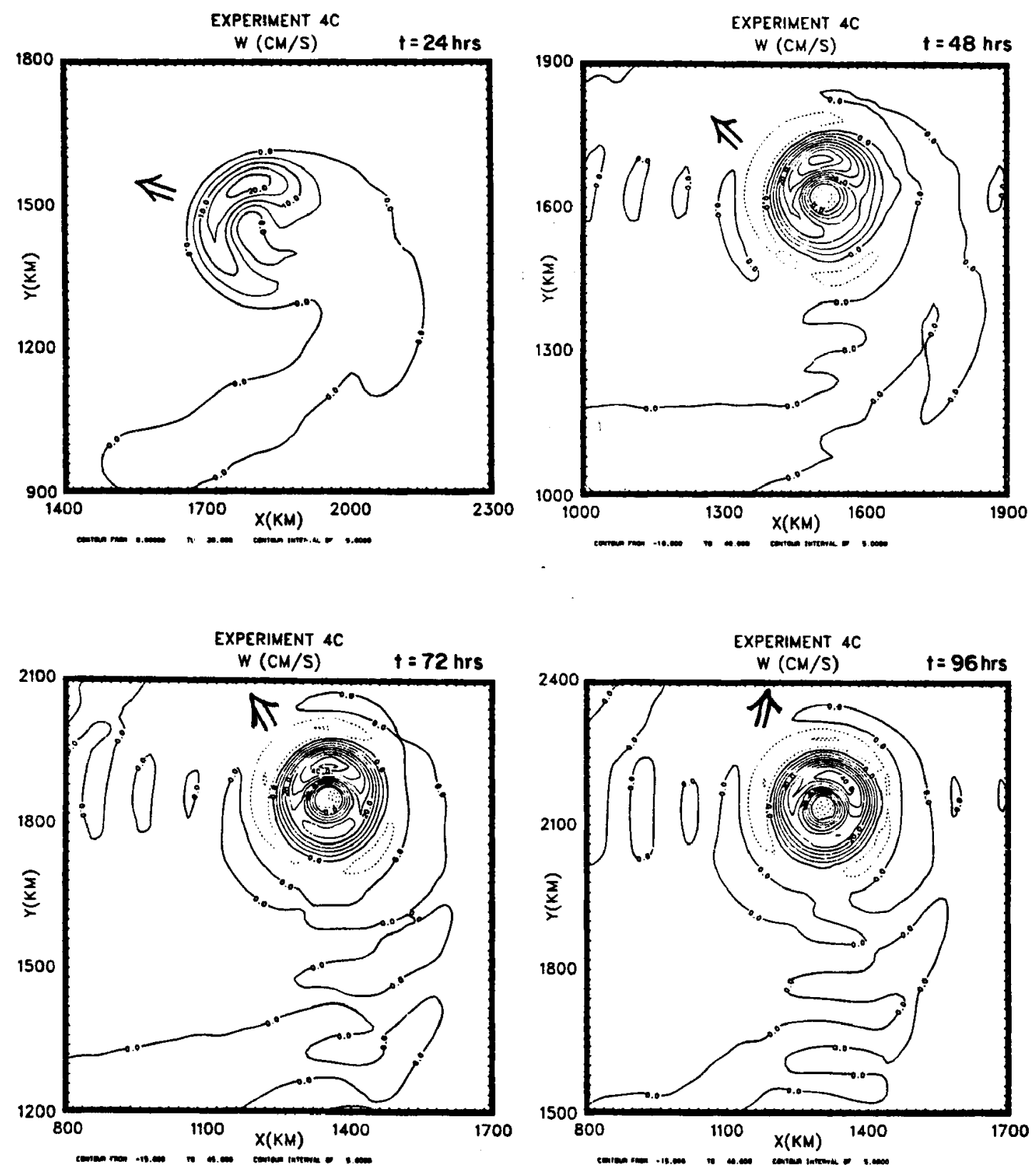

Figure 18. The vertical velocity at the top of the boundary layer $w$ at 24, 48, 72 and 96 hours for experiment $4 C$. The contour interval is $5 \mathrm{cms}^{-1}$. 
region of confluence to form on the eastern side of a cyclonic vortex. In section 4.2 , this was interpreted in terms of the eastward dispersion of the short wavelength Rossby modes. The vertical motion maximum in the right-front part of the storm, however, does not appear to be caused by the $\beta$-effect, but rather is induced by the storm motion. The translation of the storm results in stronger winds relative to the earth to the right of the direction of motion. This causes the surface drag to be asymmetric which results in asymmetric convergence and vertical motion fields. Shapiro (1983) has presented examples of the steady state flow in a slab boundary layer forced by the translation of a symmetric vortex in gradient wind balance. His results show that the translation of the vortex causes the convergence to occur in a broad arc ahead of the storm, with the convergence maximum on the right side for faster moving storms, in good agreement with Fig. 18. Shapiro also presented radar reflectivities from several storms which indicate that much of the convection occurs on the front side of the storm. Results similar to this were also presented by Miller (1958) who observed maximum low level convergence values in the right-front part of a tropical cyclone. Thus, despite the coarse horizontal and vertical resolution, the current model is capable of representing some of the smaller scale features near the storm center found in other studies and observed in nature.

The asymmetric structure of the layer 0 equivalent potential temperature deviation $\Lambda_{0}$ for this experiment can be seen in Fig. 19. In this figure, only the inner $1800 \mathrm{~km}$ by $1800 \mathrm{~km}$ of the domain is displayed and the contour interval is $4 \mathrm{~K}$. Initially, $\Lambda_{0}$ is set to a constant value of $10 \mathrm{~K}$, but gradients rapidly develop due to horizontal 

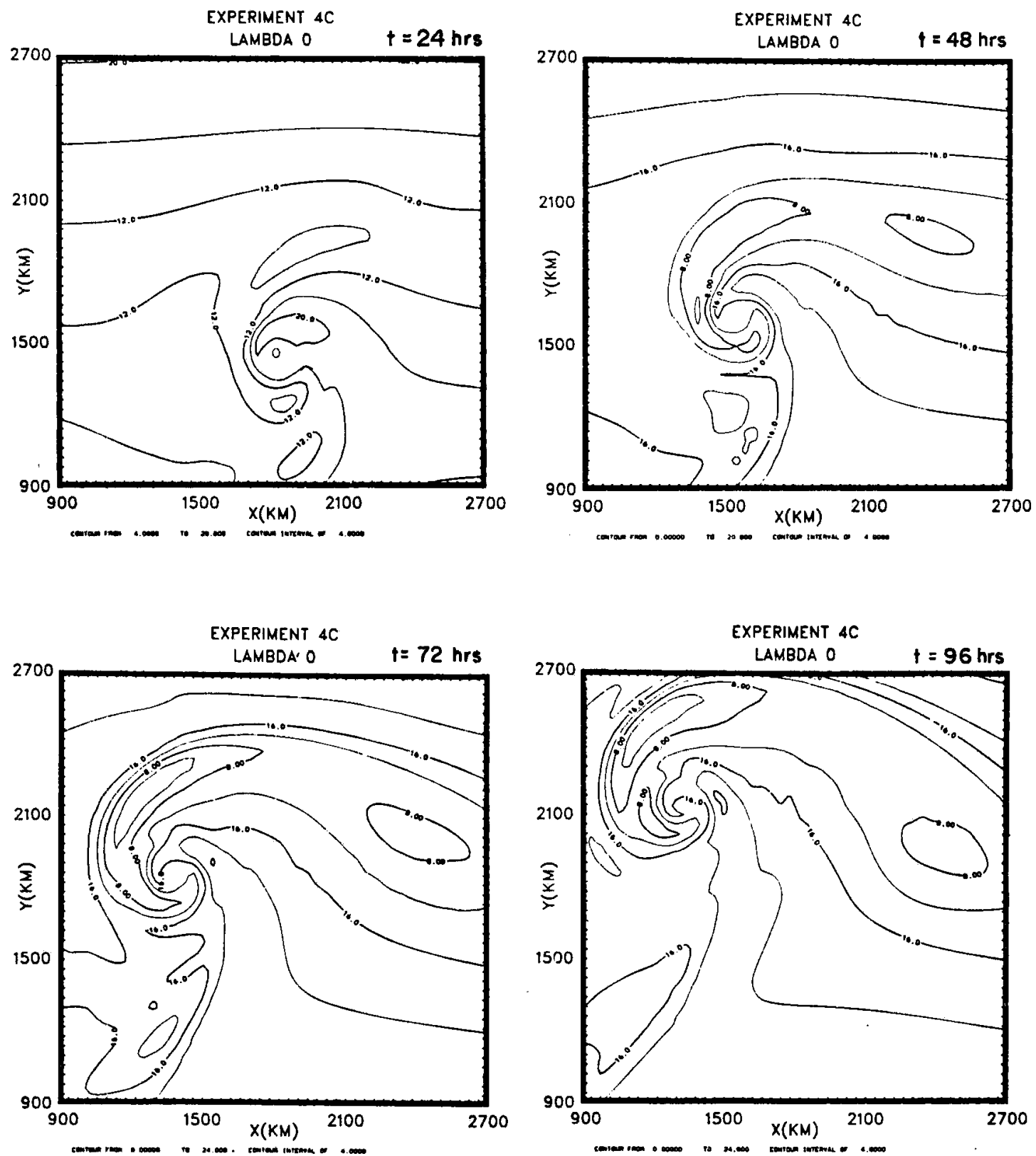

Figure 19. The boundary layer equivalent potential temperature deviation $\Lambda_{0}$ at $24,48,72$ and 96 hours for experiment $4 \mathrm{C}$. The contour interval is $4 \mathrm{~K}$. 
variations of the surface flux. Since the surface flux increases with increasing wind speed, $\Lambda_{0}$ increases the most along $y=900 \mathrm{~km}$ and $y=2700$ $\mathrm{km}$ due to the zonal wind. Between these regions the zonal wind is less so that a minimum in $\Lambda_{0}$ should develop. In addition to this, the vorticity between $y=900 \mathrm{~km}$ and $y=2700 \mathrm{~km}$ is negative due to the shear of the basic wind, so that weak subsidence occurs in this region. This causes $\Lambda_{0}$ to decrease due to the vertical advection term in (2.47). These two processes result in a north-south gradient of $\Lambda_{0}$ with a maximum at $y=900 \mathrm{~km}$, a minimum at $y=1800 \mathrm{~km}$ and a maximum at $y=2700 \mathrm{~km}$. Since the storm is initially centered at $y=1400 \mathrm{~km}$, it develops in an environment where the equivalent potential temperature decreases towards the north. This is somewhat similar to storms which form in low latitude regions with the drier subtropical regions to the north.

In Fig. 19 at 24 hours, the interaction of the storm with the large scale gradient of $\Lambda_{0}$ can be seen. The cyclonic circulation is advecting the low values of $\Lambda_{0}$ from the north around the west side of the storm and the higher values from the south around the east side of the storm. Near the storm center, $\Lambda_{0}$ increases more rapidly due to the higher wind speeds. By 48 hours, the low values of $\Lambda_{0}$ have been advected around to the south side of the storm, with the higher values on the north and east sides. This pattern can also be seen at 72 and 96 hours as the storm moves through the domain. The low values of $\Lambda_{0}$ to the west of the storm and higher values on the east side are also caused by the structure of the vertical motion field. In Fig. 18, there is subsidence to the north and west of the storm center, although the values are not large enough to be contoured. This subsidence causes a decrease of $\Lambda_{0}$ in addition to that which occurs by the advection of the low values from 
the north. On the east and south sides of the storm, particularly at 24 and 48 hours, there is rising motion associated with a convergence region induced by the $\beta$-effect. This causes $\Lambda_{0}$ to increase in addition to the increase by advection. Thus it appears that the $\beta$-effect and the interaction of the cyclone circulation with the large scale $\Lambda_{0}$ field result in higher values of $\Lambda_{0}$ on the north and east side of the storm. In the real atmosphere, this would lead to a preferential location for convection.

Fig. 19 shows the asymmetries in the $\Lambda_{0}$ field discussed above. At $t=96$ hours it can be seen that values of $\Lambda_{0}$ to the east of the storm are as high as $24 \mathrm{~K}$ while values to the northwest are as low as $4 \mathrm{~K}$. Thus, there is a gradient of $\Lambda_{0}$ of about $20 \mathrm{~K}$ across the storm. In tropical cyclones found in nature, the boundary layer equivalent potential temperature $\left(\theta_{e}\right)$ near the storm center can be up to $15 \mathrm{~K}$ warmer than the surrounding environment (e.g. Holland, 1983). Away from the storm center, however, the gradients of the boundary layer $\theta_{e}$ are usually much smaller. The model then predicts gradients of $\Lambda_{0}$ which are too large. The reason for this is that the thermodynamics of the current model are somewhat crude. As discussed previously, the assumption of a constant $\Lambda_{1}$ leads to an overestimation of the decrease of $\Lambda_{0}$ when there is subsidence in the boundary layer. Although the gradients of $\Lambda_{0}$ are somewhat overestimated, the qualitative structure of the asymetries gives some idea of how the tropical cyclone circulation can interact with the boundary layer $\theta_{e}$ field.

In summary, the asymmetric structure in this experiment is caused by several different mechanisms. The $\beta$-effect causes the storm to be elongated towards the west at large radii with a convergence region to 
the east at smaller radii. The motion of the storm induced by the mean flow and the $\beta$-effect causes asymmetries in the boundary layer vertical velocity and in the upper level flow. The basic state wind causes a north-south gradient in the $\Lambda_{0}$ field to develop which interacts with the storm circulation resulting in low values of $\Lambda_{0}$ to the west and south of the storm and higher values to the east and north.

\subsection{Transformation of a Wave-Like} Disturbance Into A Closed Vortex

The initial conditions of all of the simulations presented previously in this chapter have included a closed vortex. In this section the model is initialized with a wave-like initial condition which does not have a closed circulation. The results from this simulation (experiment 4D) will be compared to the previous experiments and also to the work of Bliss (1980) who presented a similar simulation using a finite difference model.

The initial wind field for experiment $4 \mathrm{D}$ was obtained by adding a weak symmetric vortex of the form of (4.1) to a zonal current given by (4.10). Similar to experiment $4 C$, the zonal wind varies sinusodially in $y$ with an amplitude of $-7.5 \mathrm{~ms}^{-1}$ so that there are easterlies in the southern half of the domain and westerlies in the northern half. In order that the initial wind field did not have a closed circulation, the initial symmetric vortex had a maximum tangential wind of $5 \mathrm{~ms}^{-1}$ at a radius of $300 \mathrm{~km}$ and was centered at $x=2600 \mathrm{~km}$ and $y=1400 \mathrm{~km}$ in a 4000 by $4000 \mathrm{~km}$ domain. The symmetric vortex was added only to layers 0 and 1 while the zonal wind is the same for all layers. The mass field was obtained from the wind field using the nonlinear balance equation as described previously, and $\Lambda_{0}$ was again set to a constant value of $10 \mathrm{~K}$. 
The large scale vorticity field associated with the symmetric vortex is roughly similar to, although slightly smaller than, the vorticity field of the composite easterly wave at the $700 \mathrm{mb}$ level presented by Reed et al. (1977). For the symmetric vortex, the vorticity exceeds $1 \times 10^{-5} \mathrm{~s}^{-1}$ inside a circular area with a diameter of about $800 \mathrm{~km}$ and $2 \times 10^{-5} \mathrm{~s}^{-1}$ inside an area with a $600 \mathrm{~km}$ diameter. For the composite easterly wave at the $700 \mathrm{mb}$ level, the vorticity exceeds $1 \times 10^{-5} \mathrm{~s}^{-1}$ in an eliptical region with an average diameter of about 1200 $\mathrm{km}$, and exceeds $2 \times 10^{-5} \mathrm{~s}^{-1}$ in a region with an average diameter of about $600 \mathrm{~km}$. The vorticity near the center of the symmetric vortex, however, is somewhat larger than that of the composite easterly wave. For the symmetric vortex, the vorticity exceeds $5 \times 10^{-5} \mathrm{~s}^{-1}$ inside an area with a diameter of $200 \mathrm{~km}$ and reaches a maximum of $9 \times 10^{-5} \mathrm{~s}^{-1}$ at the center, while the maximum vorticity of the composite easterly wave is about $3 \times 10^{-5} \mathrm{~s}^{-1}$.

Fig. 20 shows the wind and geopotential height fields of layer 1 for experiment $4 D$ at $t=0,24,48,72,96$ and 120 hours. The contour interval of the height field is $20 \mathrm{~m}$ which can be interpreted as a $2 \mathrm{mb}$ surface pressure interval and the entire domain is displayed. In Fig. 20 at $t=0$ it can be seen that the symmetric vortex influences an area about $2000 \mathrm{~km}$ wide. By 24 hours, the disturbance has propagated towards the west and has acquired a slight SW to NE tilt. A tilt similar to this is often observed in the lower levels of easterly waves in the eastern Atlantic region (Reed et al., 1977; Burpee, 1972, 1975). In experiment $4 \mathrm{D}$ this tilt appears to be caused by the shear of the zonal flow which advects the northern portion of the initially symmetric vortex at a slower rate than the southern portion. As the simulation 

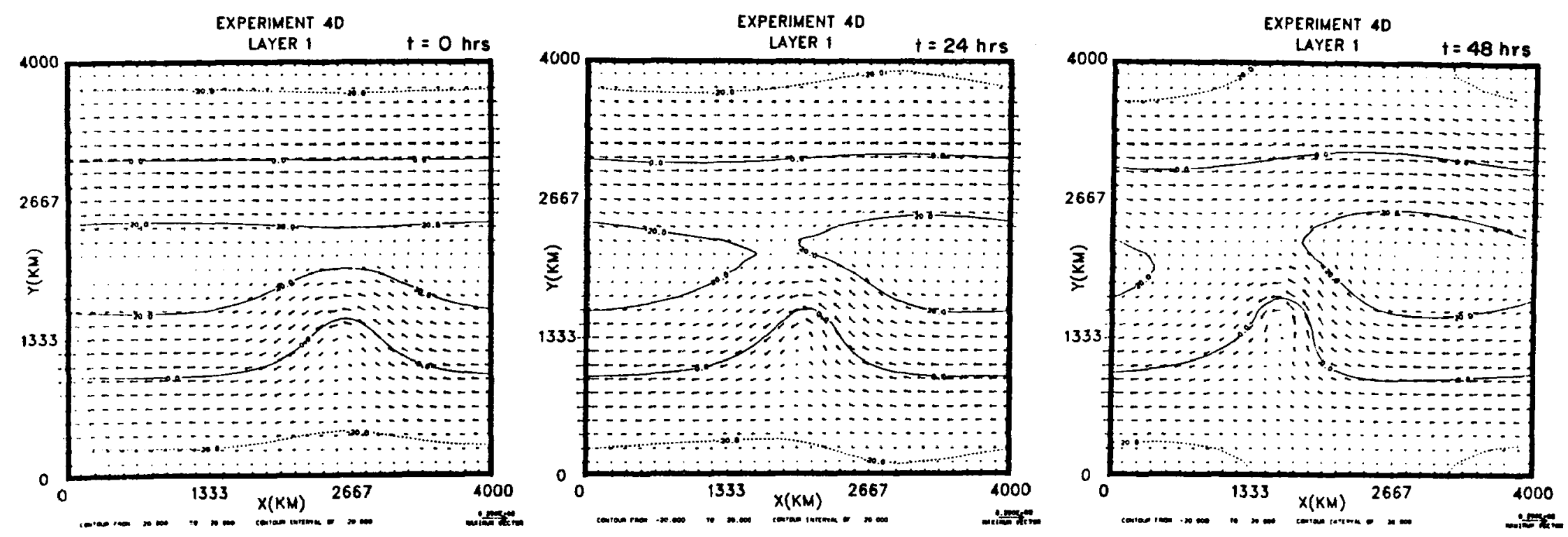

Figure 20. The layer 1 wind and geopotential height fields at $0,24,48,72,96$ and 120 hours for experiment 40 . The height contour interval is $20 \mathrm{~m}$. 

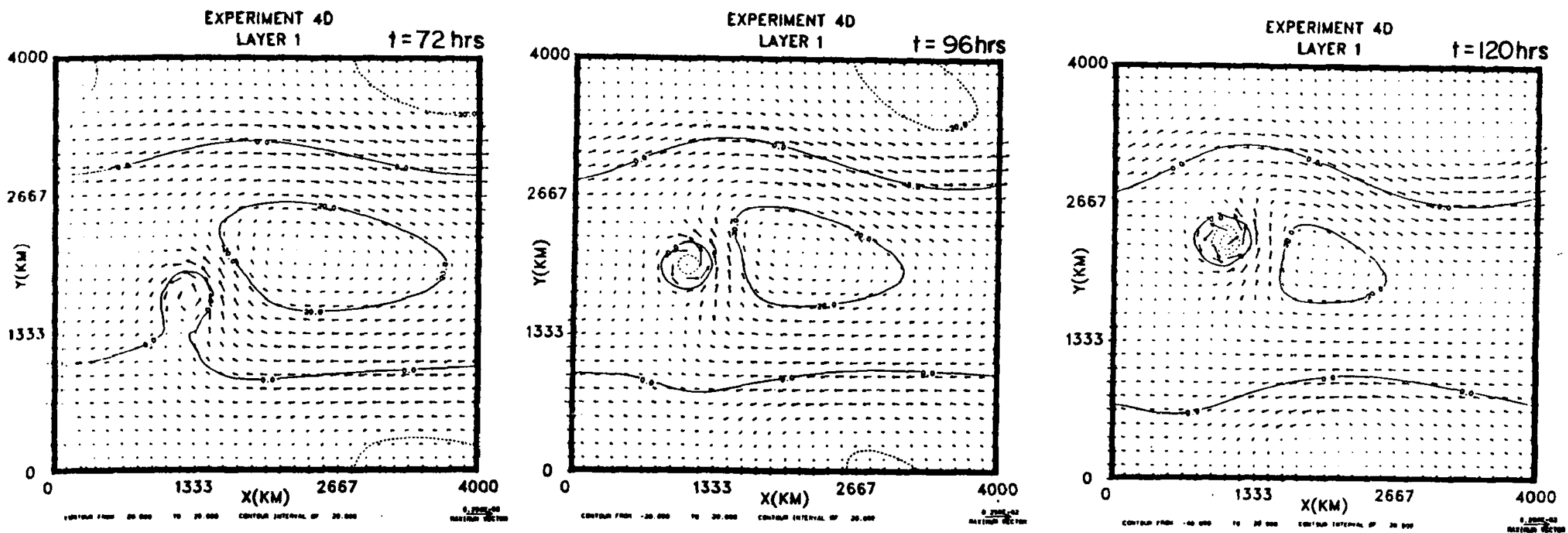

Figure 20. (continued) 
continues in Fig. 20, the disturbance becomes more concentrated as it moves towards the west. By 48 hours it appears that a closed vortex has formed which becomes more evident at later times. As the vortex develops it begins to track more towards the north and by 120 hours it has moved into the upper part of the domain where the zonal wind is westerly. When the vortex moves from the easterlies into the westerlies it causes the zonal band of high pressure in the middle of the domain to fracture into separate cells.

Fig. 21 shows the layer 2 wind and geopotential height fields for experiment $4 \mathrm{D}$ at $t=0,24,48,72,96$ and 120 hours where the contour interval of the height field is $10 \mathrm{~m}$. Initially, the upper layer contains only the zonal wind and corresponding height field. At later times, the upper level is affected as the lower level disturbance develops vertical motion and diabatic heating. By 72 hours a well developed anticyclone has formed as the lower level disturbance becomes more organized. There is also some evidence of a small area of cyclonic rotation near the horizontal location of the lower layer vortex. This is a result of the momentum transport by the diabatic term, similar to the previous experiments. This pattern continues as the lower layer vortex moves towards the north away from the easterlies. By 96 hours, the outflow in the upper layer has become highly asymmetric, with an outflow jet towards the south and southwest, similar to experiments $4 B$ and $4 C$. At 120 hours it can be seen that the outflow from the developing storm has caused an increase in the upper level easterlies southwest of the storm. Because of the periodic boundaries, the easterlies have also increased in the southeastern part of the domain. At 120 hours, it appears that the easterlies in the southeastern part of 

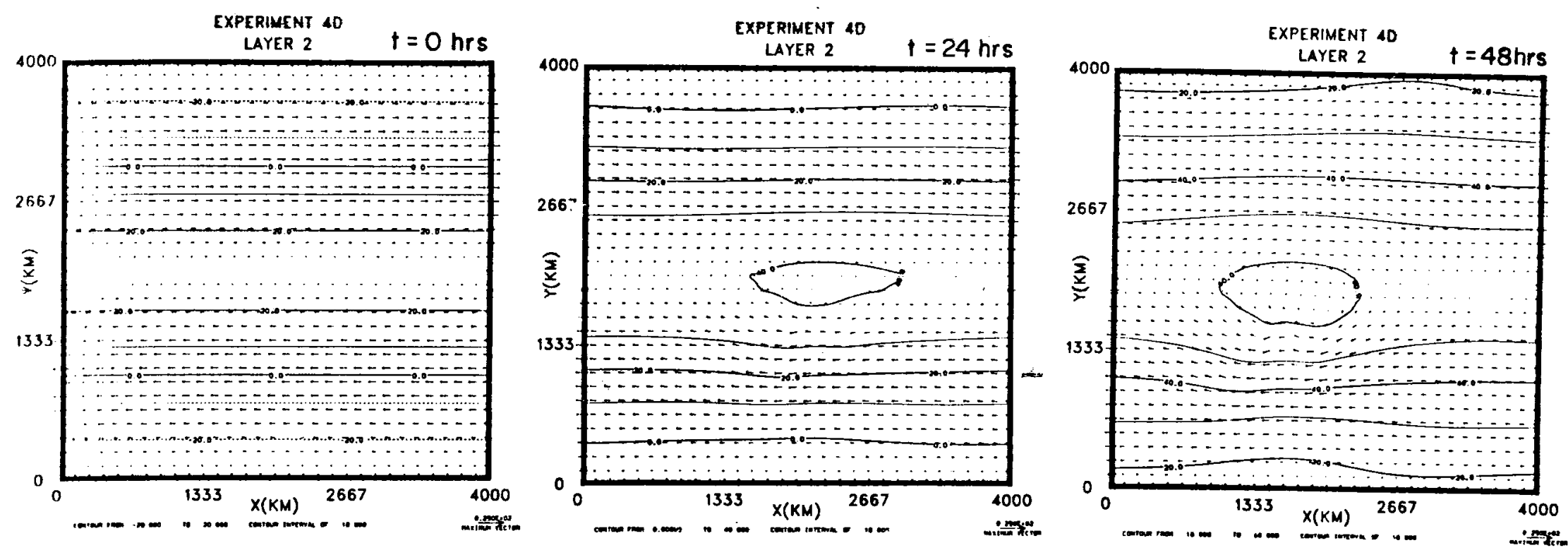

Figure 21. Same as figure 20 for layer 2. The height contour interval is $10 \mathrm{~m}$. 

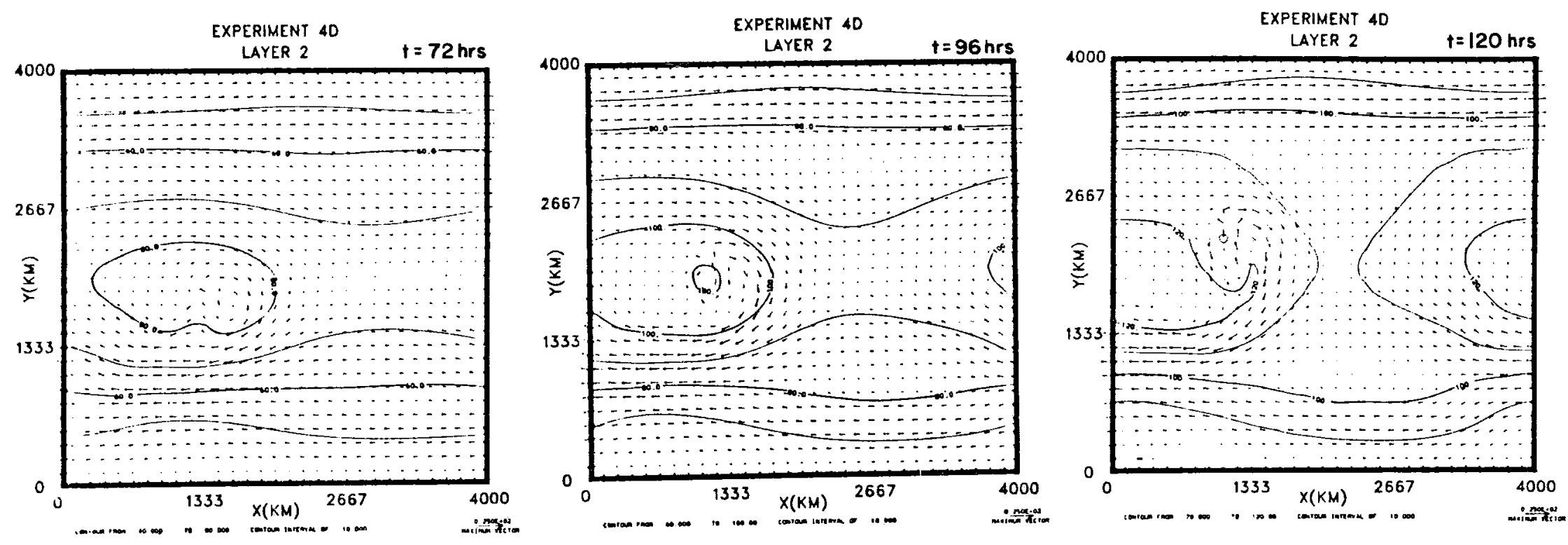

Figure 21. (continued) 
the domain may be close enough to interact with the outflow of the storm. This appears to be another limitation on the length of time the model can be integrated. Before this time, however, the periodic boundary conditions do not appear to have an influence on the solution. In order to get an idea of the intensification rate of the disturbance in experiment $4 D$, the time evolution of the minimum surface pressure deviation and maximum layer 1 wind speed are shown in Fig. 22. The track of the vorticity maximum associated with the disturbance is also shown. In Fig. 22 it can be seen that the initial intensification of the disturbance is quite slow. In the first 36 hours the maximum wind speed increases by only $1 \mathrm{~ms}^{-1}$ and the minimum surface pressure deviation decreases by less than $1 \mathrm{mb}$. During this time the vorticity center is not well defined so that the track appears to wobble somewhat. After 36 hours, a closed vortex forms and the system tracks more towards the north. By about 84 hours, the maximum layer 1 wind speed has reached $17 \mathrm{~ms}^{-1}$ which is tropical storm strength. In comparison to this, the tropical cyclones in experiments $4 A, 4 B$ and $4 C$ all reached tropical storm strength in less than 24 hours. After 96 hours, the surface pressure begins to fall more rapidly and by the end of the 6 day integration, the storm is approaching hurricane strength.

The fact that the initial development of the disturbance is so slow indicates that the organizational process is quite delicate. In the current model, the formation of convection is always linked directly to the large scale motion. In the real atmosphere, particularly in a weak disturbance, this assumption is probably not valid. As discussed by Doyama (1982), the cumulus convection in a weak large scale disturbance is not directly controlled by the large scale dynamics. For this case 
(a)

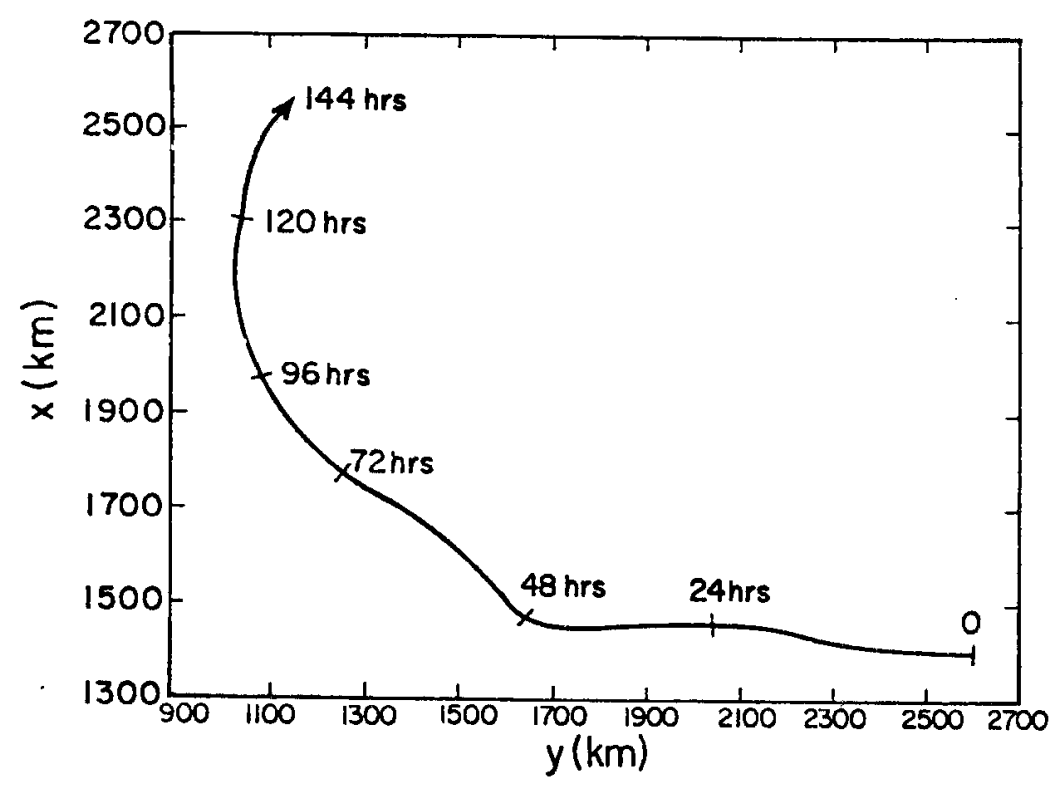

(b)

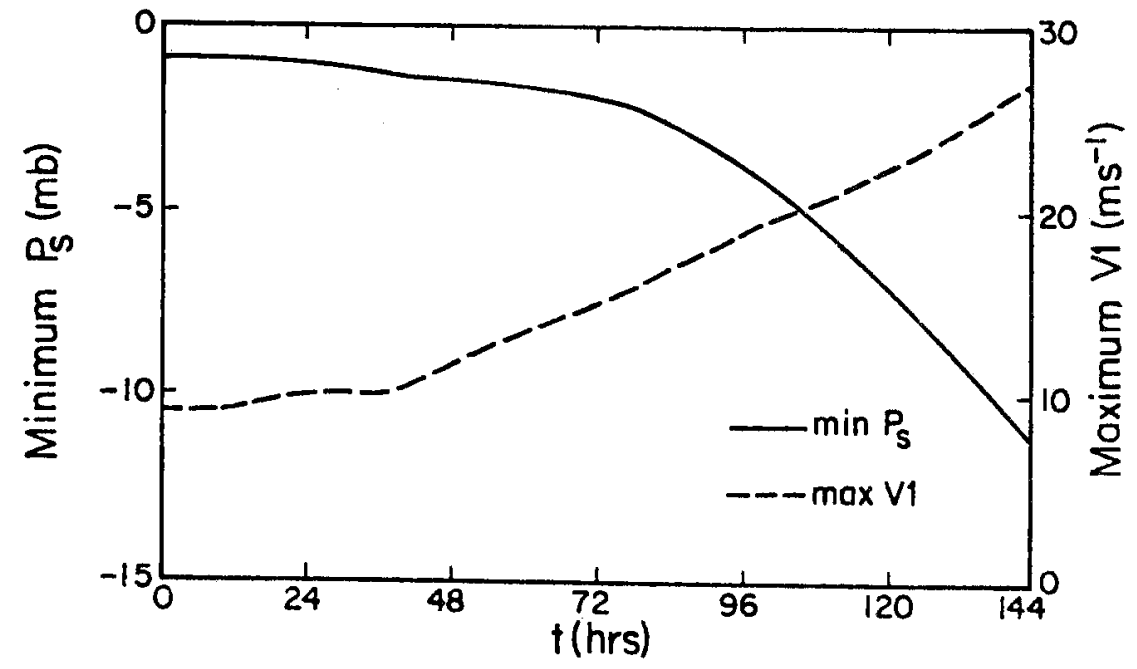

Figure 22. The track of the vorticity maximum (a) and the time evolution of the minimum surface pressure deviation and maximum layer 1 wind speed (b) for the disturbance in experiment $4 \mathrm{D}$. 
the mesoscale and cloud scale structure may also be important. As a system becomes more intense, the larger scale circulation organizes the cumulus convection so that the two are more closely linked. In the real atmosphere, then, the organizational process is even more delicate than in the model, which assumes that the cumulus convection is driven by the large scale motion for any system. This is probably one reason why it is so difficult to determine which tropical disturbances will develop into tropical cyclones.

The slow initial development and rapid deepening at later times in experiment $4 \mathrm{D}$ can be explained by considering the response of the tropical atmosphere to diabatic heat sources. The linear theory of geostrophic adjustment (e.g. Schubert et al., 1980) indicates that only a small fraction of the total energy of a heat source will produce geostrophically balanced flow. As discussed in section 4.1 , the remaining energy does not produce gravity wave motion if the time scale of the heating is long compared to the frequency of the gravity waves, but the balanced flow argument is still valid. Thus, even though the diabatic heating occurs soon after the model integration begins, the effect on the balanced flow is fairly small. Results from balanced models presented by Shapiro and Willoughby (1982) and Schubert and Hack (1982) indicate that as the inertial stability increases, a diabatic heat source becomes more efficient at producing balanced flow. For the balanced model, the inertial stability is related to the absolute vorticity. The disturbance should then begin to intensify more rapidly as the vorticity increases. In Fig. 22 the storm begins to deepen rapidly after about 96 hours. By this time, the maximum vorticity associated with the disturbance had increased by a factor of about four, 
and the storm was organized so that the diabatic heating occurred in the region of high vorticity near the storm center.

Bliss (1980) has presented model results which are quite similar to experiment 4D. Bliss used a three-dimensional version of 0oyama's model and solved the resulting equations on a doubly-periodic equatorial $\theta-$ plane using a second-order finite difference scheme. As an initial condition, Bliss used the composite easterly wave from Reed et al. (1977) where the $700 \mathrm{mb}$ wind was used for layers 0 and 1 and the $300 \mathrm{mb}$ wind was used for layer 2. The value of $\Lambda_{0}$ was initially set to a constant value of $10 \mathrm{~K}$, and the sea surface temperature was about $28^{\circ} \mathrm{C}$, which are the same values used in experiment 4D. His results showed that the easterly wave intensified and eventually formed a closed vortex after 96 hours, similar to experiment 4D. After 96 hours, however, the vortex did not continue to develop, but rather maintained a constant intensity with a maximum wind speed of about $20 \mathrm{~ms}^{-1}$. As can be seen in Fig. 22, the maximum wind speed in experiment 40 was just less than 20 $\mathrm{ms}^{-1}$ as 96 hours, but the storm continued to develop after this time. Bliss indicated that the lack of development past 96 hours was due to the initial low values of relative vorticity. This may explain some of the difference between the two model simulations since, as discussed previously, the initial condition used in experiment $4 \mathrm{D}$ had much larger values of relative vorticity near the wave axis than the composite easterly wave used by Bliss. At 96 hours, however, the maximum relative vorticity in experiment $4 \mathrm{D}$ was about $31 \times 10^{-5} \mathrm{~s}^{-1}$ compared to about $25 \times 10^{-5} \mathrm{~s}^{-1}$ in the simulation by Bliss. Thus, the maximum relative vorticity is about the same for both simulations after 96 hours. One major difference between the two simulations is the horizontal 
resolution which was used. Bliss' model had a grid spacing of $114 \mathrm{~km}$, compared to the $37 \mathrm{~km}$ resolution on the transform grid. The low resolution in the model by $B I$ iss probably prevented further intensification of the tropical cyclone. Thus, the results of the current mode 1 are similar to those of $B 1$ iss but due to the increased resolution, can better simulate the development of a tropical cyclone from a wave-like disturbance.

The simulation of the transformation of a wave into a tropical storm has also been presented by Kurihara and Tuleya (1981). Although it is difficult to compare their results using an 11-level primitive equation model to the current model results, the two simulations do have some basic similarities. In a 96 hour period the wave-like disturbance in the study by Kurihara and Tuleya transforms into a closed vortex as the maximum relative vorticity increases from $4.3 \times 10^{-5}$ to $23.7 \times 10^{-5} \mathrm{~s}^{-1}$, the maximum low level winds increase from 10 to $17 \mathrm{~ms}^{-1}$ and the minimum surface pressure decreases by $5.8 \mathrm{mb}$. These are comparable to the results from the current model where the maximum relative vorticity increases from $7.6 \times 10^{-5}$ to $31.0 \times 10^{-5} \mathrm{~s}^{-1}$, the maximum layer 1 winds increase from 9 to $19 \mathrm{~ms}^{-1}$ and the surface pressure decreases by $2.8 \mathrm{mb}$ in 96 hours. The wave at $700 \mathrm{mb}$ in Kurihara and Tuleya's model also develops a SW to NE tilt similar to the current model and observations, and the storm which forms forces an anticyclone in the upper levels with a small cyclonic region near the storm center. This indicates that although the current model has very crude vertical resolution, it can capture some of the basic structure found in more complicated models and in nature for the case of the transformation of a wave into a closed vortex. 
CHAPTER 5

THE INFLUENCE OF THE LARGE-SCALE FLOW ON TROPICAL CYCLONE INTENSIFICATION

The size and intensity of tropical cyclones found in nature vary considerably from storm to storm. For example, Merrill (1982) has presented a climatology of storm size in terms of the radius of the outer closed isobar (ROCI). His results show that the average ROCI for Atlantic tropical cyclones is $3^{\circ}$ latitude, although values between $1^{\circ}$ and $9^{\circ}$ have been observed. Merrill has also shown that tropical cyclones in the Pacific are about twice as large in areal extent as Atlantic storms. Tropical cyclones also tend to be clustered in time. Gray (1979) has shown that more than $2 / 3$ of all the tropical cyclones during a twenty year period developed in less than $1 / 3$ of the corresponding storm seasons. These results imply that the intensification and resulting structure of tropical cyclones are affected by many factors. Many modeling studies have investigated the effects of sea surface temperature and other factors which might influence the cumulus convection. There have also been some studies which show that the large scale environment can affect tropical disturbances. Shapiro (1977) has shown that the vorticity of an easterly wave can increase as it passes through an environment with large positive vorticity. Challa and Pfeffer (1980) have presented results from an axisymmetric tropical cyclone model which showed that the storm structure is sensitive to eddy fluxes of angular momentum 
which could be induced by the large scale environment. In their study, however, the effect of the large scale environment is specified so that the interaction with the storm circulation is not simulated. Tuleya and Kurihara (1981) have shown that the large scale environment can affect the genesis of a tropical storm from an easterly wave. In this chapter, results from five simulations are presented which show that the large scale flow field in both the upper and lower layers can affect the structure and intensification rate of the model tropical cyclone. The effects of horizontally sheared mean flows in the upper and lower layers are considered in sections 5.1 and 5.2. In section 5.3, the interaction of a developing tropical cyclone with a mid-latitude trough is simulated. Table 4 gives a summary of the five experiments to be discussed in this chapter.

\subsection{The Effect of Horizontal Wind Shear In the Lower Layers}

In this section the effect of a horizontally sheared basic state wind in layers 0 and 1 is investigated. In order to keep the simulations as simple as possible, a symmetric vortex is added to an antisymmetric zonal wind field and $\beta$ is set to zero, so that the vortex does not move. The basic state wind field is given by $(4.10)$ with $U_{i}$

set to $-7.5 \mathrm{~ms}^{-1}$ or $7.5 \mathrm{~ms}^{-1}$ in layers 0 and 1 for experiment $5 \mathrm{Al}$ or 5B1. The symmetric vortex given by (4.1) with $V_{m}=10 \mathrm{~ms}^{-1}$ and $r_{m}=100 \mathrm{~km}$ is added to the zonal wind in layers 0 and 1 and the mass field is found from the nonlinear balance equation (4.5). The symmetric vortex is centered on a 3600 by $3600 \mathrm{~km}$ domain so that the zonal wind is antisymmetric with respect to the vortex position. From (4.10) it can be seen that the vorticity of the zonal wind is given by 


\section{TABLE 4.}

Summary of the numerical simulations presented in chapter 5

\begin{tabular}{|c|c|c|c|c|}
\hline & Initial Vortex & $\begin{array}{c}\text { Initial Basic } \\
\text { State Zonal Wind }\end{array}$ & $\begin{array}{l}\text { Integration } \\
\text { Length (days) }\end{array}$ & $\begin{array}{l}\text { Coriolis } \\
\text { Parameter }\end{array}$ \\
\hline $5 A 1$ & $\begin{array}{l}\text { Symmetric vortex in } \\
\text { layers } 0 \text { and } 1 \\
V_{m}=10 \mathrm{~ms}^{-1} \underset{r_{m}}{ }=100 \mathrm{~km}\end{array}$ & $\begin{array}{l}\text { Anticyclonic shear } \\
\text { near vortex in } \\
\text { layers } 0 \text { and } 1\end{array}$ & 4 & $B=0$ \\
\hline $5 B 1$ & $\begin{array}{l}\text { Symmetric vortex in } \\
\text { layers } 0 \text { and } 1 \\
v_{m}=10 \mathrm{~ms}^{-1} r_{m}=100 \mathrm{~km}\end{array}$ & $\begin{array}{l}\text { Cyclonic shear } \\
\text { near vortex in } \\
\text { layers } 0 \text { and } 1\end{array}$ & 4 & $\beta=0$ \\
\hline $5 A 2$ & $\begin{array}{l}\text { Symmetric vortex in } \\
\text { layers } 0 \text { and } 1 \\
v_{m}=10 \mathrm{~ms}^{-1} r_{m}=100 \mathrm{~km}\end{array}$ & $\begin{array}{l}\text { Anticyclonic shear } \\
\text { near vortex in } \\
\text { layer } 2\end{array}$ & 4 & $\beta=0$ \\
\hline $5 \mathrm{~B} 2$ & $\begin{array}{l}\text { Symmetric vortex in } \\
\text { layers } 0 \text { and } 1 \\
v_{m}=10 \mathrm{~ms}^{-1} \quad r_{m}=100 \mathrm{~km}\end{array}$ & $\begin{array}{l}\text { Cyclonic shear } \\
\text { near vortex in } \\
\text { layer } 2\end{array}$ & 4 & $\beta=0$ \\
\hline $5 C$ & $\begin{array}{l}\text { Symmetric vortex in } \\
\text { layers } 0 \text { and } 1 \\
V_{m}=10 \mathrm{~ms}^{-1} \quad r_{m}=150 \mathrm{~km}\end{array}$ & $\begin{array}{l}\text { Basic state in } \\
\text { a } 11 \text { layers, with } \\
\text { trough in layer } 2\end{array}$ & 5 & $\beta \neq 0$ \\
\hline
\end{tabular}




$$
-\frac{\partial \bar{u}_{i}}{\partial y}=-u_{i}\left(\frac{2 \pi}{L_{y}}\right) \cos \left(\frac{2 \pi y}{L_{y}}\right) .
$$

For $L_{y}=3600 \mathrm{~km}$ and $\left|U_{j}\right|=7.5 \mathrm{~ms}^{-1}$, the vorticity of the zonal wind has extrema of magnitude $1.3 \times 10^{-5} \mathrm{~s}^{-1}$ at $y=0, L_{y} / 2$ and $L_{y}$. The vorticity of the zonal wind in the region of the vortex $\left(y=L_{y} / 2\right)$ is negative for experiment $5 A 1$ and positive for experiment 5B1. Except for the addition of the zonal wind, experiments $5 A 1$ and $5 B 1$ are identical to experiment $4 A$, so that the three experiments can be compared.

Fig. 23a shows the minimum surface pressure deviation for experiments $4 A, 5 A 1$ and $5 B 1$. Initially, the surface pressure deviation is lower than experiment $4 A$ for $5 B 1$ and higher for $5 A 1$. This is due to the pressure field associated with the basic state wind. By 24 hours, the difference between the pressure deviations has decreased which indicates that the storm with the anticyclonic basic state vorticity has deepened the most rapidly while the positive basic state vorticity case has deepened the slowest. By about 36 hours, the pressure of the storm with the anticyclonic basic state wind (5Al) begins to level off while the other two storms continue to deepen. By about 60 hours, the pressure of the storm with no basic state wind $(4 A)$ begins to drop less rapidly while the pressure of the storm with the cyclonic basic state wind (5B1) drops more rapidly. By the end of the 96 hour integration, the pressure in experiment $5 B 1$ is almost as low as for experiment $4 A$, and is dropping more rapidly.

The maximum layer 1 wind speeds for each of these experiments behave similar to the surface pressure deviations, as can be seen in Fig. 23b. Initially the wind speed in experiment 5AI increases the most rapidly, but levels off after about 36 hours. The wind speed in 

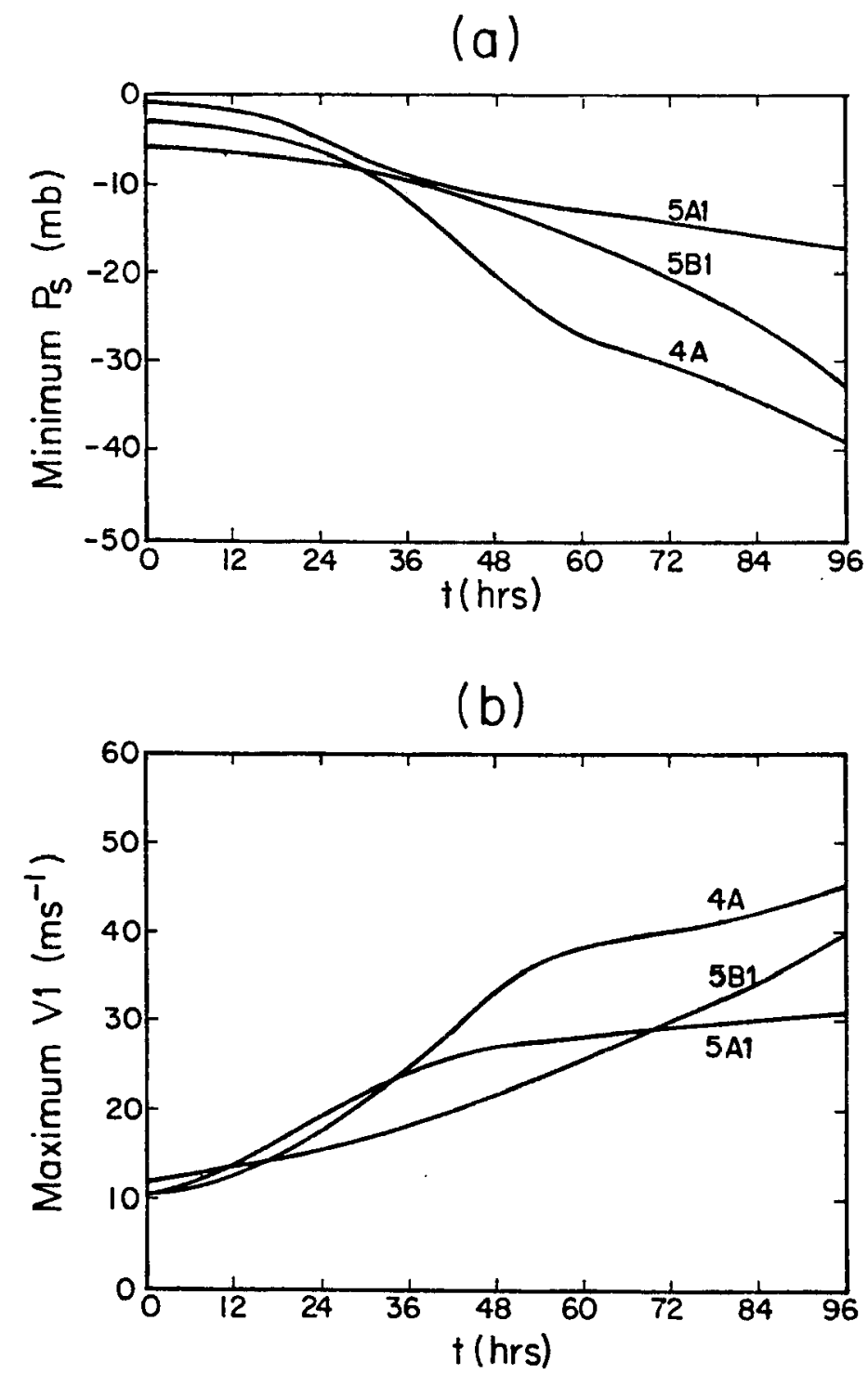

Figure 23. The time evolution of the minimum surface pressure deviation (a) and maximum layer 1 wind speed for experiments $4 A, 5 A 7$ and $5 B T$. 
experiment $4 \mathrm{~A}$ then increases the most rapidly until about 60 hours when the wind in experiment 5B1 increases more rapidly. By the end of the 96 hour integration, the wind speed for experiment $5 B 1$ is almost as high as for experiment $4 \mathrm{~A}$, and is increasing more rapidly.

These results indicate that the presence of the basic state wind in the lower layers affects the intensification rate of the storm. An anticyclonically sheared environment appears to favor rapid initial growth which levels off after a fairly short time, while a cyclonically sheared environment slows the initial growth rate, but enables the storm to continue to intensify over a longer period of time.

The fact that the addition of a basic state wind with anticyclonic vorticity caused the storm to intensify more rapidly may seem somewhat surprising in light of the results presented by Schubert and Hack (1982). They have shown that in an axisymmetric balanced model, a diabatic heat source becomes more efficient at producing balanced flow as the inertial stability increases. Since the inertial stability increases as the relative vorticity increases, it should then be expected that the addition of a background vorticity which is negative would decrease the inertial stability and the efficiency of the heat source, which should decrease the intensification rate. In order to determine why this does not occur, an inertial stability parameter $\mu$ and the diabatic heat source $Q$ were calculated using azimuthally averaged values of the dependent variables. For an axisymmetric vortex, the inertial stability $\mu^{2}$ is given by

$$
\mu^{2}=\left[\frac{1}{r} \frac{\partial}{\partial r}\left(r V_{T}\right)+f\right]\left[\frac{2 V_{T}}{r}+f\right]
$$

where $V_{T}$ is the tangential wind. For each of the simulations in this 
section the value of $\mu$ was calculated from (5.2) using azimuthally averaged values of the layer 1 tangential wind. For convenience, the values of $\mu$ were normalized by the Coriolis parameter $f_{0}$ so that $\mu$ has the value of unity when the fluid is at rest. Equation (5.2) is actually only valid for an axisymmetric vortex, while experiments 5A1 and $5 \mathrm{~B} 1$ are asymmetric. The storms are fairly symmetric close to the center, however, so that the value of $\mu$ should give some indication of the efficiency of the diabatic heat source.

Fig. 24 shows the azimuthally averaged values of $\mu$ and $Q$ as a function of radius at $0,24,48$ and $72 \mathrm{hrs}$ for experiments $4 \mathrm{~A}, 5 \mathrm{Al}$ and 5Bl. At $t=0$ in Fig. $24, Q$ is zero since the initial conditions used were nondivergent, and the inertial stability parameters appear to be quite similar in all three experiments. This is because for small $r$ the vorticity of the vortex is much larger than that of the basic state. At larger radii, however, the vorticity of the vortex is small so that the effect of the basic state becomes more important. For example, at $r=300$ $\mathrm{km}$ (not shown in Fig. 24) the parameter $\mu$ has a value of $1.0,0.8$ and 1.3 for experiments $4 A, 5 A 1$ and $5 B 1$ respectively. By 24 hours, a vertical motion field has developed so that $Q$ becomes nonzero. At this time it can be seen that $Q$ is larger for experiment $5 A 1$ which had the anticyclonically sheared basic state wind. Also at this time, the maximum value of $Q$ occurs at a smaller radius for experiment $5 A 1$, so that the inertial stability is larger in the region of the forcing. Assuming the arguments from the axisymmetric model are valid, this indicates that the efficiency of the diabatic heat source for producing balanced flow will be greater for experiment 5Al. Since the magnitude and the efficiency of the diabatic heat source are larger for experiment 

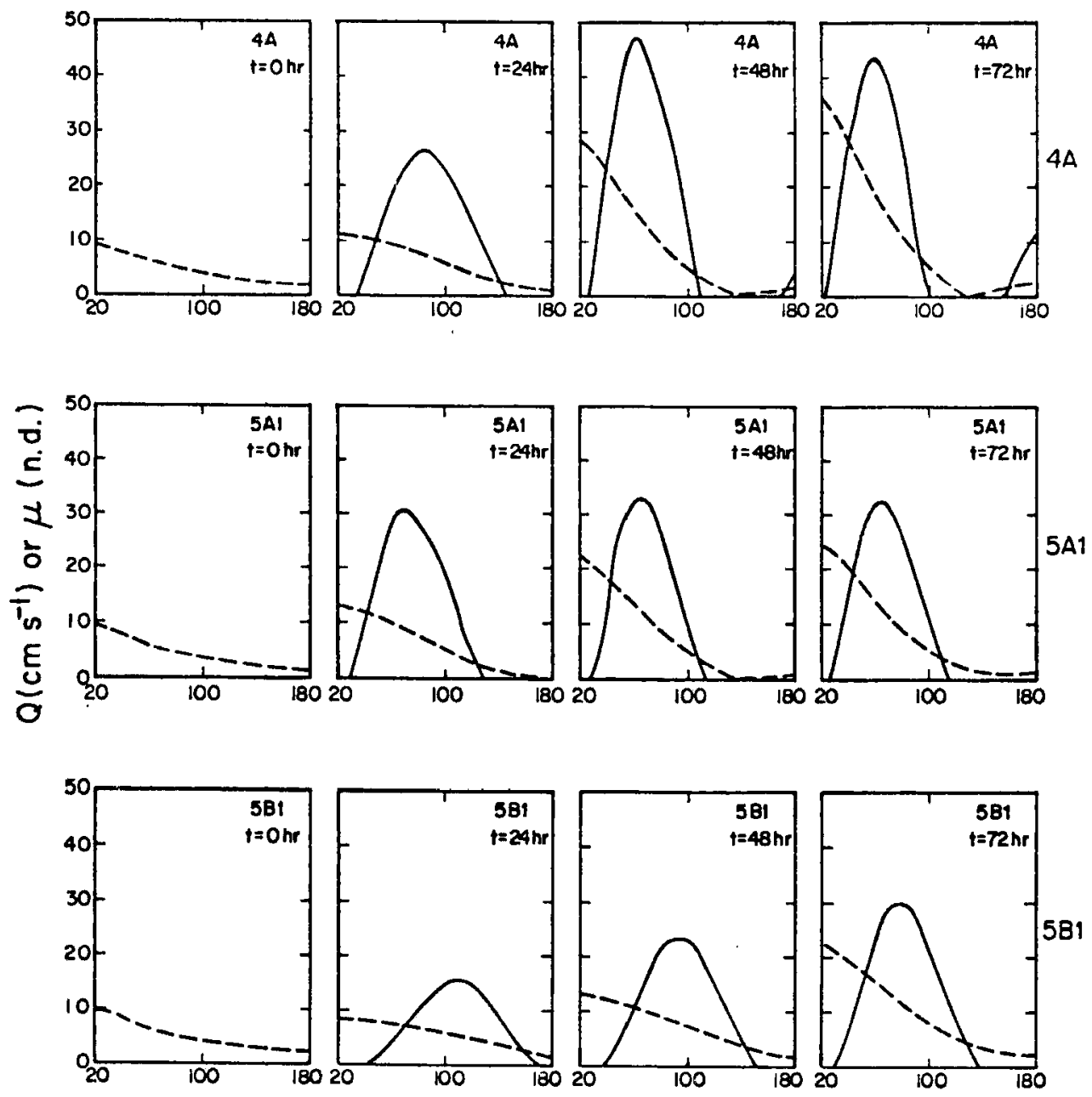

$r(\mathrm{~km})$

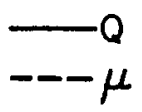

Figure 24. The azimuthally averaged diabatic term $Q$ and inertial stability parameter $\mu$ as a function of radius at 0,24 , 48 and 72 hours for experiments $4 A, 5 A 1$ and $5 B 1$. 
$5 \mathrm{Al}$, the vortex should intensify more rapidly as was the case. A similar argument holds for experiment 5B1 where the lower magnitude and efficiency of $Q$ at $t=24 \mathrm{hr}$ resulted in the slower intensification.

The initial development of the $Q$ fields shown in Fig. 24 can be explained by again considering the inertial stability. Since $Q$ is given by $n^{+}{ }^{+}$and $n$ varies slowly as a function of $r$, the structure of $Q$ is largely determined by the structure of $w$. In an axisymetric sense, the vertical velocity field at the top of the boundary layer is a result of the radial circulation which develops because of the surface drag. Since the vorticity of the vortex is cyclonic, radial inflow develops in the boundary layer. In the axisymmetric model, the inertial stability is a measure of the resistance to radial motion. As discussed previously, the inertial stability parameter is reduced or increased by the basic state wind in experiments $5 \mathrm{~A} 1$ and $5 \mathrm{~B} 1$ respectively. Thus, it appears that the radial circulation in experiment $5 \mathrm{~A} 1$ can penetrate closer to the center of the vortex due to the reduced inertial stability at fairly large radii. When this flow reaches the region of large inertial stability near the vortex center, convergence of the radial wind occurs which results in vertical motion. In contrast to this, it appears that the larger inertial stability values away from the vortex center in experiment $5 B 1$ reduces the magnitude of the radial circulation before it reaches the vortex center so that the vertical motion occurs at larger radii. These results indicate that the radial structure of the inertial stability is important for determining the location of the parameterized heat source, and that results from studies of tropical cyclone intensification with specified forcing must be interpreted carefully. 
After 24 hours in Fig. 24 it can be seen that $Q$ increases for experiments $4 A$ and $5 B 1$, but has leveled off in experiment $5 A 1$. This is probably related to the large scale vorticity field. As the diabatic forcing becomes active, radial inflow develops in layers 0 and 1 which concentrates the background vorticity, which then spins up the vortex. As the vortex spins up, the radial circulation increases resulting in larger diabatic forcing. Since the large scale vorticity is less in experiment $5 A 1$, it is then more difficult for the vortex to intensify by this process. In contrast to this, after about 72 hours when the diabatic heat source in experiment $5 B 1$ is established, the vortex develops more rapidly than for experiments $4 A$ or $5 A 1$ as was seen in Fig. 23.

In addition to the intensification rate, the structure of the tropical cyclones differed for each of the experiments discussed in this section. Fig. 25a shows the radial structure of the azimuthally averaged layer 1 tangential wind at 96 hours for each experiment. In Fig. 25a it can be seen that the vortex in experiment $5 A 1$ is less intense than for the other experiments (smaller maximum tangential wind) but is also much smaller than the other two vorticies. Experiments $4 \mathrm{~A}$ and $5 B 1$, however, appear to produce the same size vorticies, although experiment $4 \mathrm{~A}$ produced a more intense storm by 96 hours. The fact that the storms in experiment $4 A$ and $5 B 1$ are the same size is probably a result of both the larger diabatic forcing in experiment 4A (shown in Fig. 24) and the larger background vorticity of experiment 5B1. Since the diabatic forcing in experiment $4 \mathrm{~A}$ was larger before 96 hours, the radial inflow in layers 0 and 1 out to about $800 \mathrm{~km}$ was larger (not shown) so that the vortex should have spun up more. Since the 
(a)

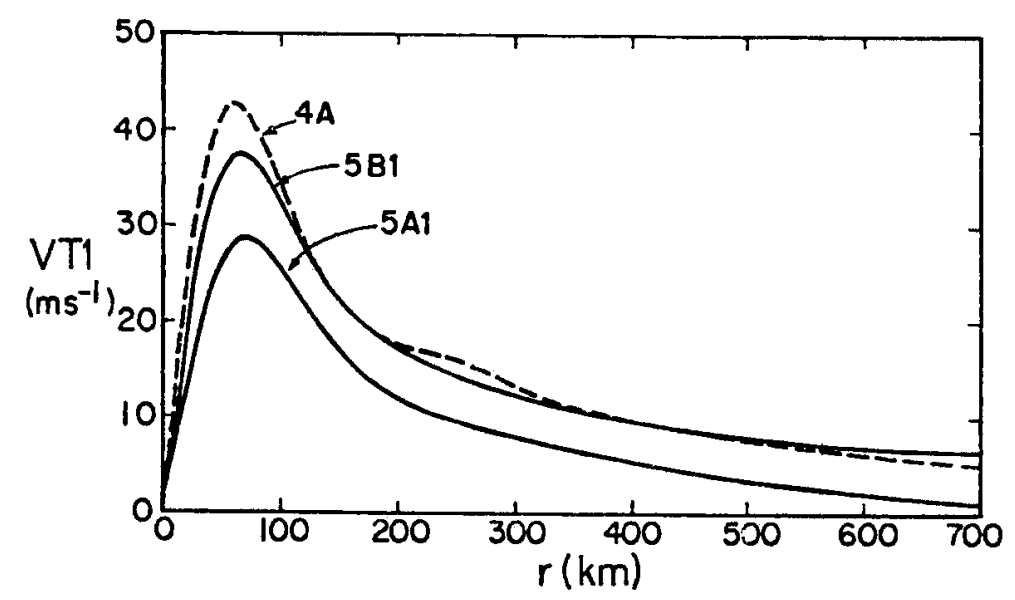

(b)

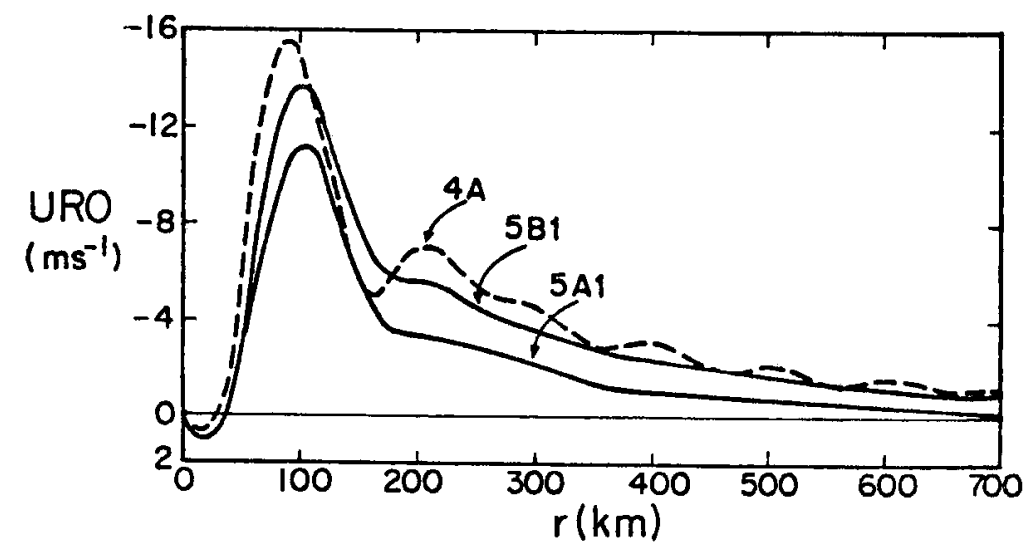

Figure 25. The azimuthally averaged layer 1 tangential wind (a) and layer 0 radial wind (b) at 96 hours for experiments $4 A, 5 A 1$ and $5 B 1$. 
background vorticity was larger in experiment $5 B 1$, however, not as much inflow was needed to spin up the storm, so that the resulting vorticies were about the same size by 96 hours. In contrast to this, both the diabatic heat source after 24 hours and the background vorticity were smaller for experiment $5 A 1$ so that the vortex was considerably smaller.

Part of the difference in the vortex structure shown in Fig. 25a can be accounted for simply by adding a symmetric vortex to the different basic state wind fields. Experiment $5 \mathrm{Al}$ had westerlies to the north and easterlies to the south which subtracts from the tangential wind, while the opposite was true for experiment 5B1. The basic state does not, however, directly change the radial wind field. In order to get a better idea of how large an area is affected by the tropical cyclones, the azimuthally averaged layer 0 radial wind fields at 96 hours for each experiment are shown in Fig. 25b. The structure of the radial winds for each experiment is similar to the tangential wind structure. In Fig. 25b it can be seen that the radial wind for experiment $5 A 1$ is weaker at all radii than for experiments $4 A$ or $5 B 1$ which indicates that this storm is considerably smaller. This figure also shows that at 96 hours, the radial wind in layer 0 outside of about $200 \mathrm{~km}$ is still slightly less for experiment $5 B 1$ than for $4 A$, which is probably a result of the stronger diabatic heating in experiment $4 A$ as discussed previously.

Figure 26 shows the two-dimensional structure of the layer 1 wind and geopotential height fields at 96 hours for experiments $4 A, 5 A 1$ and 5B1. Despite the presence of the basic state winds, the tropical cyclones which develop are quite symmetric near the center for experiments $5 A 1$ and $5 B 1$. This lends some support to the arguments used 

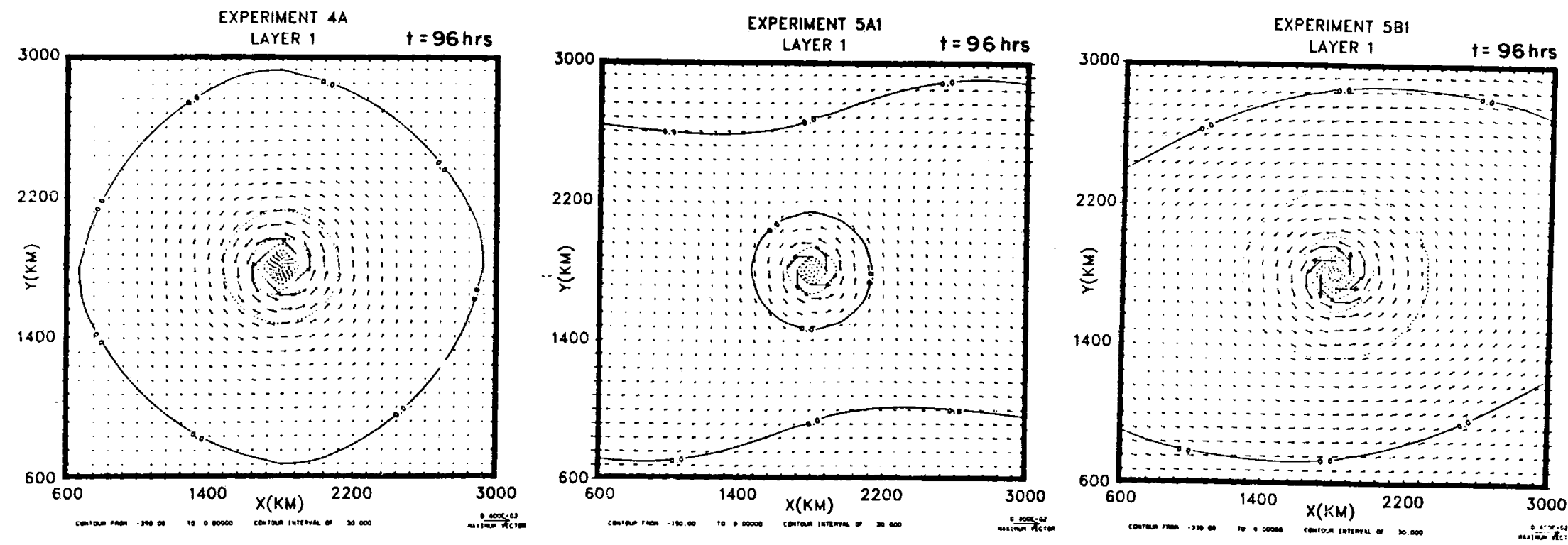

Figure 26. The layer 1 wind and geopotential height fields at 96 hours for experiments $4 A, 5 A 1$ and $5 B 1$. The height contour interval is $30 \mathrm{~m}$. 
to explain the intensification rates of the storms which were based on results from an axisymmetric model. This figure also shows that the vortex for experiment $5 A 1$ is smaller than for $5 B 1$ or $4 A$, as was apparent in Fig. 25.

\subsection{The Effect of Horizontal Wind Shear}

In The Upper Layer

In this section, the simulations presented in the previous section are repeated except that the basic state wind appears only in the upper layer. The basic state wind is again given by (4.10) with $U_{i}$ set to $-7.5 \mathrm{~ms}^{-1}$ or $7.5 \mathrm{~ms}^{-1}$ in layer 2 for experiment 5A2 or 5B2 respectively, and the vortex given by (4.1) with $v_{m}=10 \mathrm{~ms}^{-1}$ and $r_{\mathrm{m}}=100 \mathrm{~km}$ is added in layers 0 and 1 . Analogous to experiments $5 A 1$ and $5 B 1$, the basic state wind in the upper layer has anticyclonic or cyclonic shear in the region of the vortex for experiments $5 A 2$ and $5 B 2$ respectively. $A 11$ other parameters are identical to those used for experiment $4 \mathrm{~A}$ so that experiments $4 A, 5 A 2$ and $5 B 2$ can be compared.

Fig. 27 shows the minimum surface pressure deviation and the maximum layer 1 wind speed for experiments $4 A, 5 A 2$ and 5B2. From this figure it can be seen that the tropical cyclone in experiment 5B2 intensifies the most rapidly both in terms of minimum surface pressure and maximum layer 1 wind speed, while experiment 5 A2 intensifies the slowest. By the end of the 96 hour integration, the storm in experiment $5 \mathrm{~B} 2$ has a minimum surface pressure deviation about $10 \mathrm{mb}$ lower and maximum wind speed about $5 \mathrm{~ms}^{-1}$ higher than for experiment 4A. This effect of the basic state wind in the upper layer appears to be contradictory to some observational studies of tropical cylcones. For example Yanai (1964) and Colon and Nightingale (1963) have indicated 
(a)

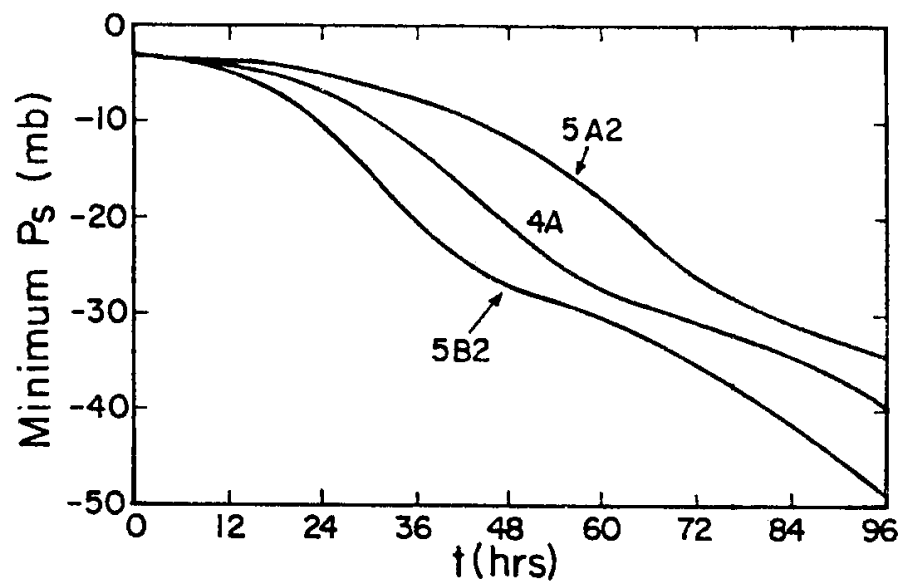

(b)

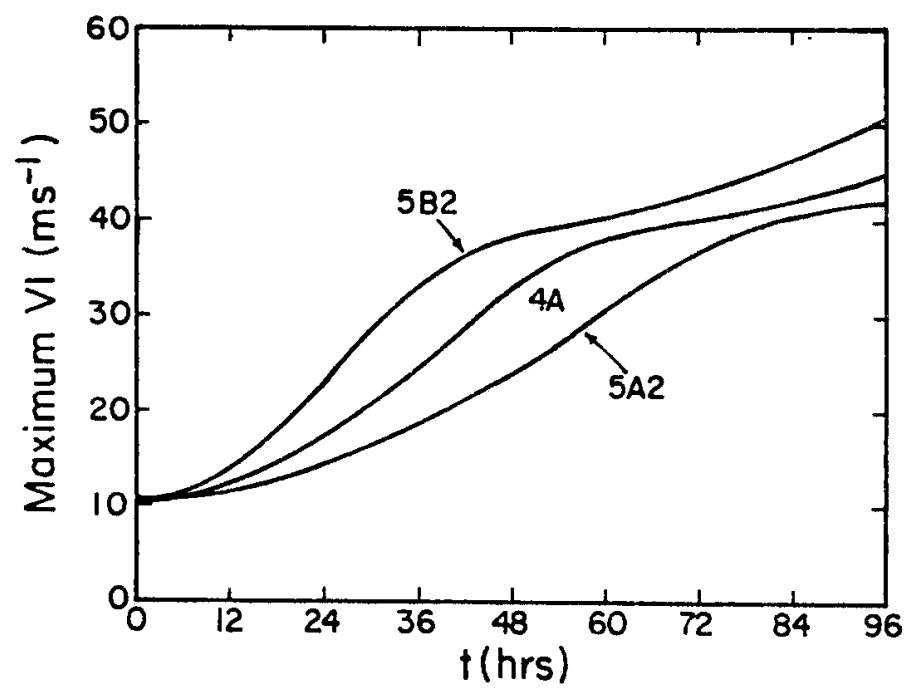

Figure 27. The time evolution of the minimum surface pressure deviation (a) and maximum layer 1 wind speed (b) for experiments $4 \mathrm{~A}, 5 \mathrm{~A} 2$ and $5 \mathrm{~B} 2$. 
that a preexisting upper level anticyclone might be favorable for tropical cyclone intensification. In the model simulations, however, the storm in experiment $5 A 2$ which had an anticyclonically sheared basic state wind above the vortex intensified slower than experiment $4 \mathrm{~A}$, while the storm in experiment $5 \mathrm{~B} 2$ with a cyclonically sheared basic state wind intensified more rapidly. This difference in the intensification rates in the three simulations appears to be related to two different processes which are discussed below.

The first process is related to the convective stability parameter n. As shown in (2.44), $n$ is a function $\Lambda_{0}, \Lambda_{1}$ and $\Lambda_{2}$, where $\Lambda_{1}$ is a constant for all the simulations. Initially, $\Lambda_{0}$ is the same for each simulation since it is set to a constant value of $10 \mathrm{~K}$. The initial value of $\Lambda_{2}$, however, is proportional to $h_{2}$ which is different for each simulation. Since the nonlinear balance equation was used to determine the mass fields, $\phi_{2}$ is zero, positive or negative above the vortex in experiments $4 A, 5 A 2$ or $5 B 2$ respectively. The $\phi_{1}$ fields are the same for each experiment since the wind field in layer 1 is the same, so that $h_{2}$ is larger in experiment $5 A 2$ and smaller in experiment 5B2 (since $h_{2}$ is proportional to $\left.\phi_{2}-\phi_{1}\right)$. This indicates that initially $n$ is the largest for experiment $5 \mathrm{~B} 2$ and the smallest for experiment $5 \mathrm{~A} 2$ in the region of the vortex. At the initial time, the average value of $\eta$ inside a radius of $200 \mathrm{~km}$ was found to be $1.51,1.31$ and 1.72 for experiments $4 \mathrm{~A}, 5 \mathrm{~A} 2$ and 5B2. Since $n$ is larger for experiment 5B2 it should be expected that this storm would intensify more rapidly since this implies that $Q$ will be larger when the boundary layer vertical velocity field develops. The decreased value of $h_{2}$ above the vortex in experiment 5B2 is analogous to a colder temperature in the upper leve1. The increase in $n$ 
then simulates the decreased vertical stability caused by the colder temperatures aloft. In the real atmosphere, an upper level cyclonic region implies colder temperatures aloft while an upper level anticyclonic region implies warmer temperatures aloft. If a developing tropical disturbance moves under an upper level anticyclone, the warmer upper level temperatures might then suppress the convective activity. From this point of view, a preexisting upper level anticyclone might not be favorable for intensification if this stabilizing effect dominates over any possible dynamic effects.

The larger initial values of $\eta$ in experiment 5B2 probably account for its more rapid initial development. As this tropical cyclone intensifies, however, $h_{2}$ increases because of the diabatic forcing so that after about 12 hours, the average value of $\eta$ inside a $200 \mathrm{~km}$ radius is smaller than for the other two experiments. In Fig. 27 the storm in experiment $5 B 2$ continues its rapid intensification until about 36 hours, and intensifies further after 72 hours. This continued intensification appears to be related to the structure of the upper layer wind field. Fig. 28 shows the wind and geopotential height fields in layer 2 at 72 hours for experiments $4 \mathrm{~A}, 5 \mathrm{~A} 2$ and $5 \mathrm{~B} 2$. For experiment $4 \mathrm{~A}$, a cyclonic region has formed in the upper layer near the vortex center, as discussed previously, with a large symmetric anticyclonic region away from the center. A symmetric cyclonic region can also be seen near the vortex center for experiments $5 \mathrm{~A} 2$ and $5 \mathrm{~B} 2$, but the anticylconic regions away from the vortex center are highly asymmetric. In experiment 5A2, the outflow has interacted with the basic state wind so that two closed anticycionic vorticies have formed to the northeast and southwest of the vortex. In experiment 5B2, two large outflow jets have formed to the 

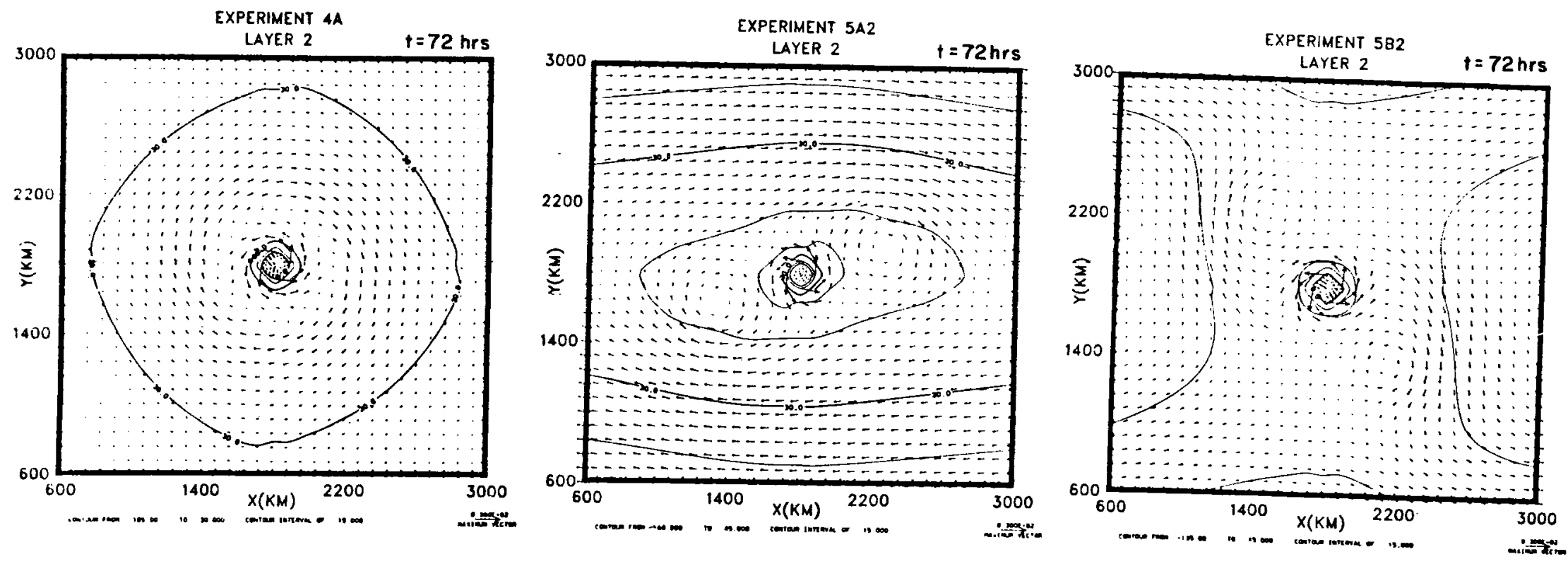

Figure 28. The layer 2 wind and geopotential height fields at 72 hours for experiments $4 A, 5 A 2$ and $5 B 2$. The height contour interval is $15 \mathrm{~m}$. 
northwest and southeast of the vortex center. It appears that the outflow jets in experiment 5B2 export mass far from the system which allows the surface pressure to decrease so that the intensification rate is accelerated. For experiment 5A2 it appears that the outflow structure is less favorable for the export of mass because of the closed anticyclonic vorticies so that the intensification rate is reduced.

These results show that the structure of the outflow region of a tropical cyclone is very sensitive to the large scale environment. These results also show that the upper layer structure can affect the intensification rate of the storm, with some indication that the establishment of outflow jets is favorable for development.

Although the upper layer flow shown in Fig. 28 was highly asymmetric, the lower layers remained quite symmetric, so that azimuthally averaged winds are representative of the entire wind field. Fig. 29 shows the azimuthally averaged layer 1 tangential wind and layer 0 radial wind at 96 hours for experiments $4 A, 5 A 2$ and $5 B 2$. In this figure it can be seen that the basic state wind in experiment 5B2 has caused the layer 1 vortex to be more intense and also somewhat larger. The boundary layer inflow also is stronger in both the inner and outer regions of the storm for experiment 582 .

Although the amplitudes of the tangential and radial wind for each experiment shown in Fig. 29 are different, the radial structure is quite similar. This indicates that the basic state wind in the upper level primarily affects the intensifictaion rate of the tropical cyclones, but does not have a large effect on the horizontal structure. This is somewhat different from the results in the previous section which indicated that the basic state in the lower levels affects the 
(a)

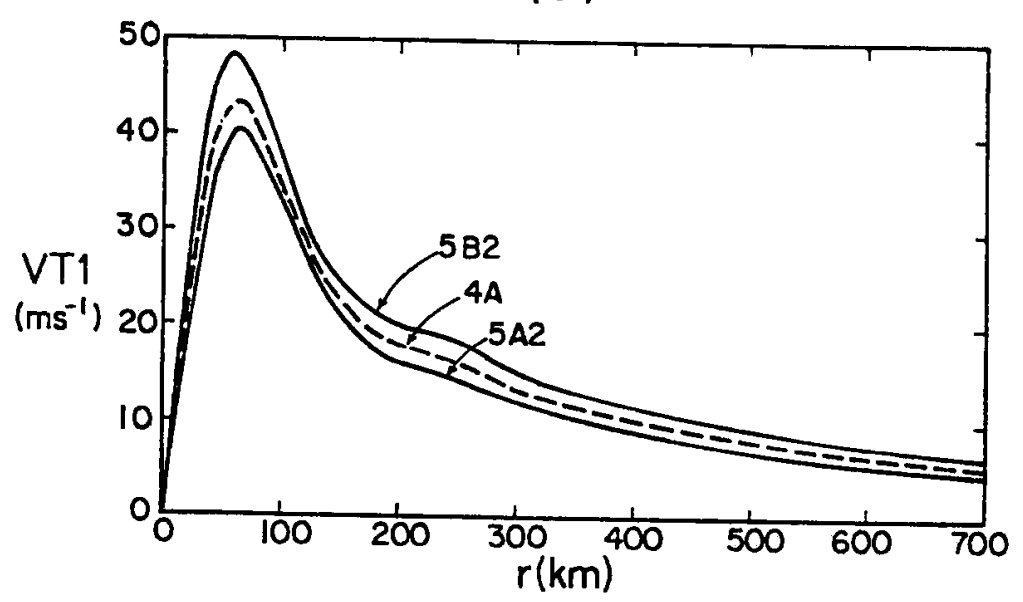

(b)

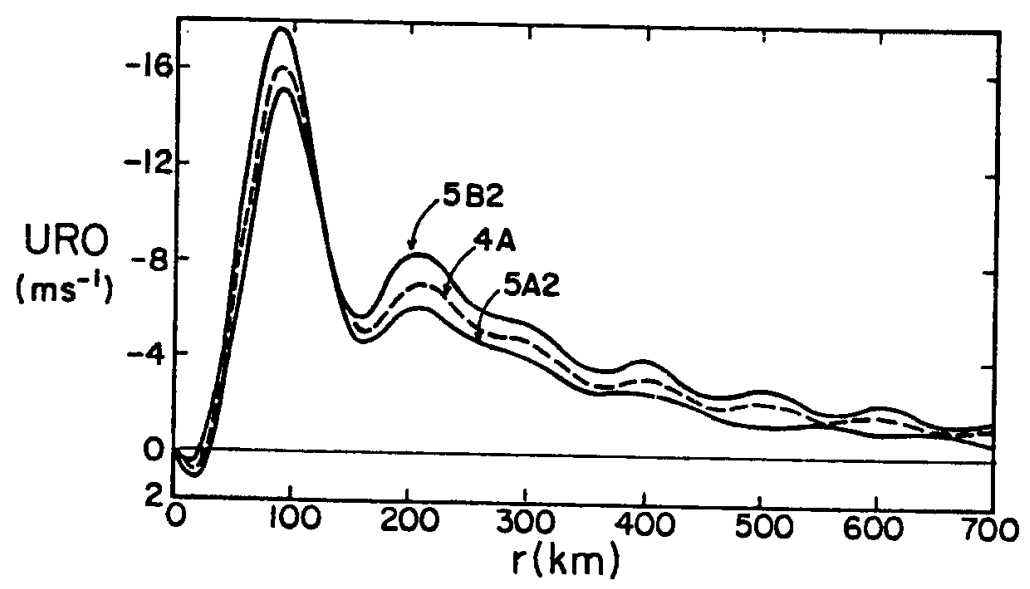

Figure 29. The azimuthally averaged layer 1 tangential wind (a) and layer 0 radial wind (b) at 96 hours for experiments $4 A, 5 A 2$ and $5 B 2$. 
intensification rate and the horizontal structure of the wind field in the lower layers.

\subsection{Interaction With A Trough In The Upper Layer}

Tropical cyclones typically form in low latitudes and move towards the west under the influence of the tropical easterlies (e.g. Gray, 1979). After an initial westward track storms often turn towards the north, and sometimes recurve toward the east as they approach the midlatitude westerlies. Tropical cyclones which move towards the midlatitude westerlies often interact with synoptic scale disturbances which occur in this region. In the previous two sections it was shown that the large scale zonal wind field affected the intensification rate and the structure of a stationary tropical cyclone on an f-plane. In this section results are presented from an experiment (5C) with a somewhat more realistic environment which contains an idealized upper level trough on a $\beta-p l a n e$.

The initial conditions used in experiment $5 C$ are identical to those used in experiment $4 C$, except that an area of positive vorticity is added to layer 2 in the northern part of the domain. Similar to experiment $4 C$, the basic state zonal wind is given by (4.10) with $U_{i}$ equal to $-7.5 \mathrm{~ms}^{-1}$ for all layers, so that the southern half of the domain contains easterlies, and the northern half contains westerlies. The symmetric vortex given by (4.1) with $v_{m}=10 \mathrm{~ms}^{-1}$ and $r_{\mathrm{m}}=150 \mathrm{~km}$ was added to layers 0 and 1 at $x=2200 \mathrm{~km}$ and $y=1400 \mathrm{~km}$ on a 3600 by $3600 \mathrm{~km}$ domain. In order to simulate an upper level wave, a symmetric vortex given by 


$$
V=V_{m}\left(\frac{r}{r_{m}}\right) \exp \frac{1}{2}\left[1-\left(\frac{r}{r_{m}}\right)^{2}\right]
$$

was added in the upper layer at $x=1800 \mathrm{~km}$ and $y=2250 \mathrm{~km}$. In (5.3), the maximum tangential wind $v_{m}$ was specified to be $7 \mathrm{~ms}^{-1}$ at a radius $r_{m}$ of $450 \mathrm{~km}$. The vortex was positioned in the part of the domain where the basic state wind is from the west so that a wave-like disturbance results.

Fig. 30 shows the layer 2 wind and geopotential height fields for experiment $4 \mathrm{C}$ and $5 \mathrm{C}$ at $0,24,48$ and 72 hours. As mentioned previously, the initial conditions are identical for each experiment except for the added upper layer disturbance in experiment 5C, which can be seen in Fig. 30. The position of the layer 1 streamfunction minimum associated with the tropical cyclone is indicated in Fig. 30 by the synoptic symbol for a hurricane. At $t=0$, the layer 2 wind field in experiment $4 C$ consists of the zonal flow, while there is a trough to the northwest of the storm in experiment 5C. By 24 hours, the storms in each experiment have moved towards the west and have begun to develop. For both cases a small region of cyclonic rotation has formed near the storm center with some evidence of an anticyclonic vortex towards the northeast. By this time, the trough in experiment $5 \mathrm{C}$ has moved toward the east so that the tropical cyclone is just to the east of the trough axis. At 48 hours, both storms have intensified further (the maximum layer 1 wind speed is about $30 \mathrm{~ms}^{-1}$ for both experiments) and the outflow patterns have become more well defined. At this time it can be seen that the structure of the outflow for each storm is quite different. In experiment $4 C$, the initial outflow is towards the north, but rapidly acquires anticyclonic curvature resulting in a closed 

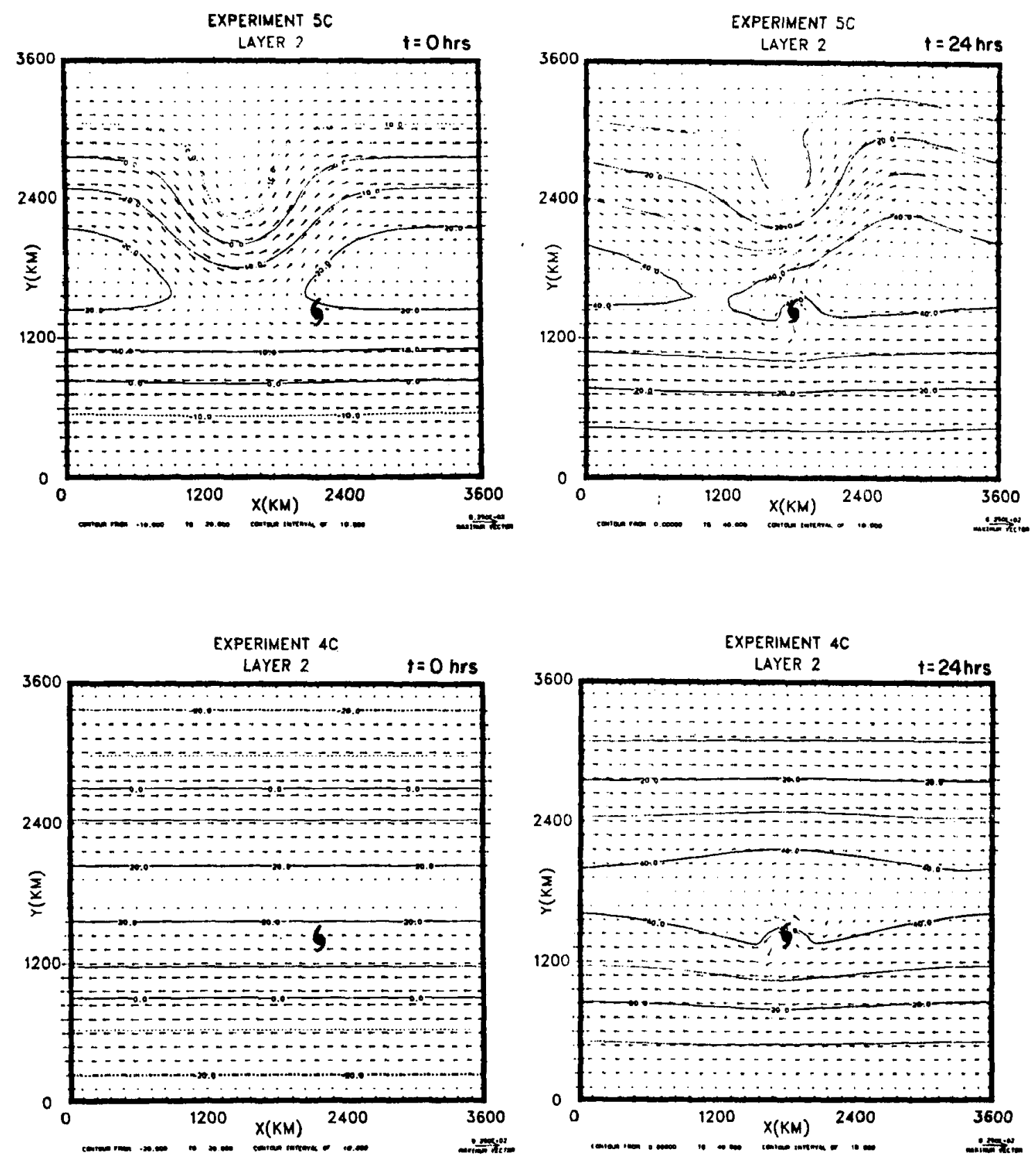

Figure 30. The layer 2 wind and geopotential height fields at $0,24,48$ and 72 hours for experiment $5 \mathrm{C}$ (upper) and $4 C$ (lower). The height contour interval is $10 \mathrm{~m}$ and the layer 1 position of the tropical cyclone is indicated by the synoptic symbol for a hurricane. 

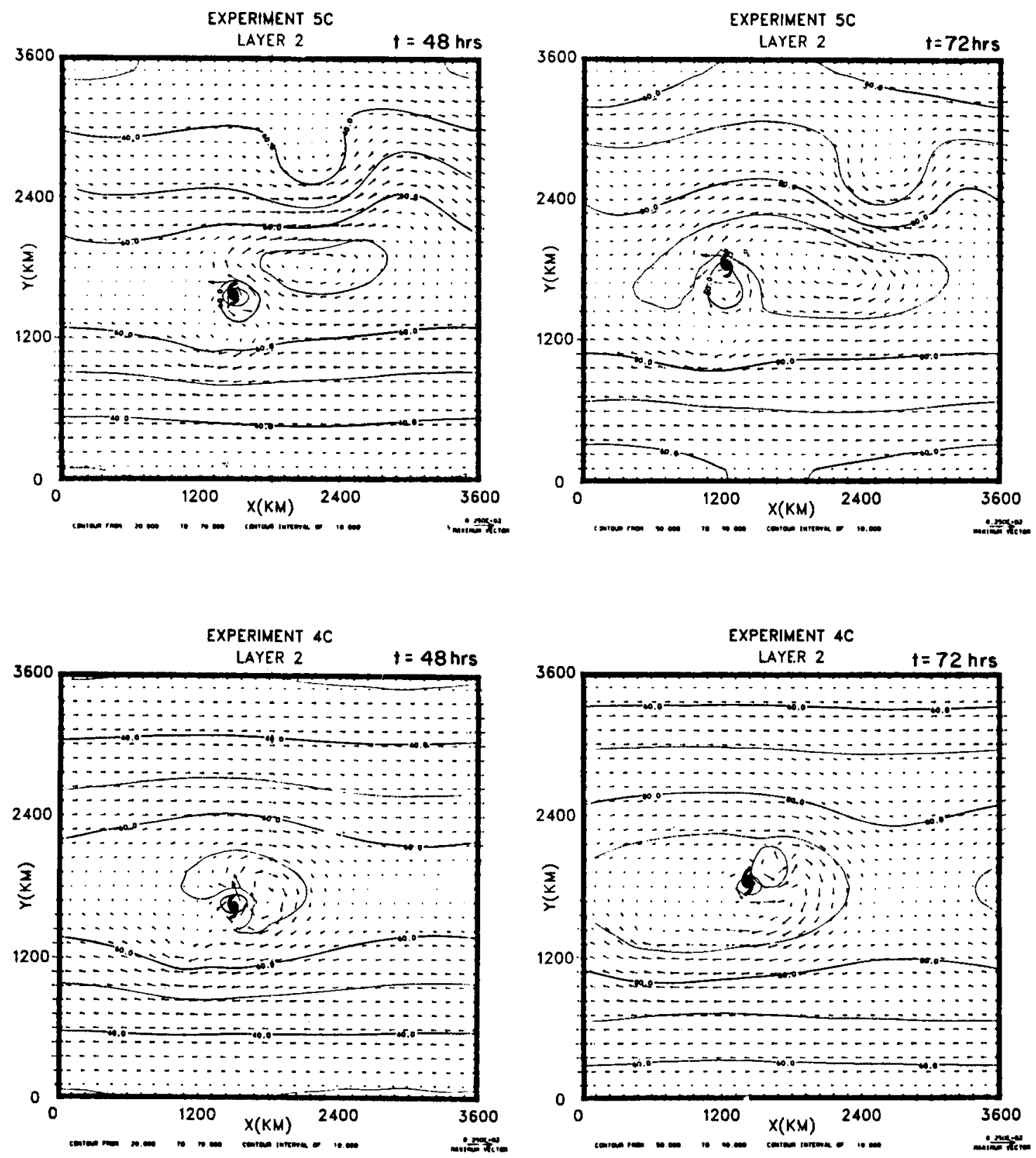

Figure $30 . \quad$ (Continued) 
anticyclonic vortex to the east of the storm center. It also appears that much of the mass eventually flows towards the south and connects with the easterly current. In experiment $5 C$ the initial outflow is also towards the north, but then turns towards the east, apparently in response to the upper level trough. Because of this, much of the mass flows towards the north and northeast and connects with the westerly current. At 72 hours, the outflow structure is similar to that at 48 hours as the trough in experiment $5 \mathrm{C}$ continues to move towards the east. At this time it appears that the outflow in experiment $4 C$ has acted to increase the easterly wind to the south of the storm center while the outflow has increased the westerly wind to the northwest of the storm center in experiment $5 C$.

The above results indicate that mid-latitude disturbances can influence the structure of the outflow layer. In the current case, the upper level trough acted to change the direction of the main branch of the outflow. As shown by Chen (1983), the structure of the outflow from tropical cyclones is highly variable. These results indicate that a large part of the variability can probably be explained by considering differences in the large scale environment in the upper levels.

The upper level trough in experiment $5 \mathrm{C}$ also had an effect on the track of the tropical cyclone. Fig. 31a shows the difference between the position of the layer 1 streamfunction minimum for experiments $5 \mathrm{C}$ and $4 C$. In this figure, a positive $\Delta x$ or $\Delta y$ indicates that the storm in experiment $5 C$ was east or north of the storm in experiment $4 C$. The actual position of the storm in experiment $5 \mathrm{C}$ could be found by adding $\Delta x$ and $\Delta y$ at any given time to the position of the storm in experiment $4 \mathrm{C}$ shown in Fig. 16. This figure shows that in the first 24 hours, the 
(a)

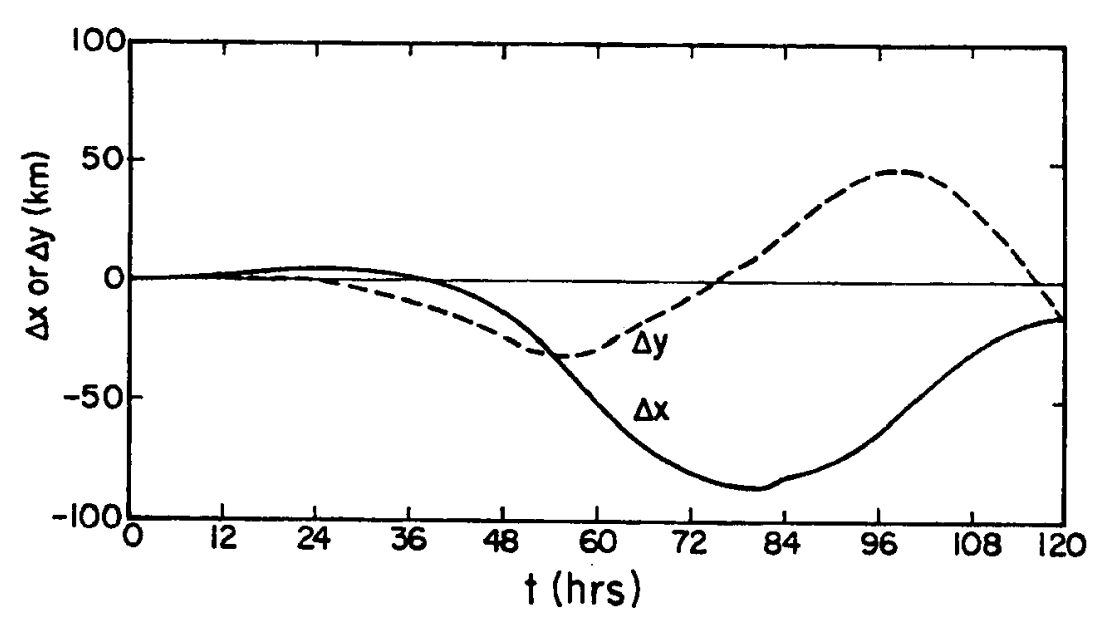

(b)

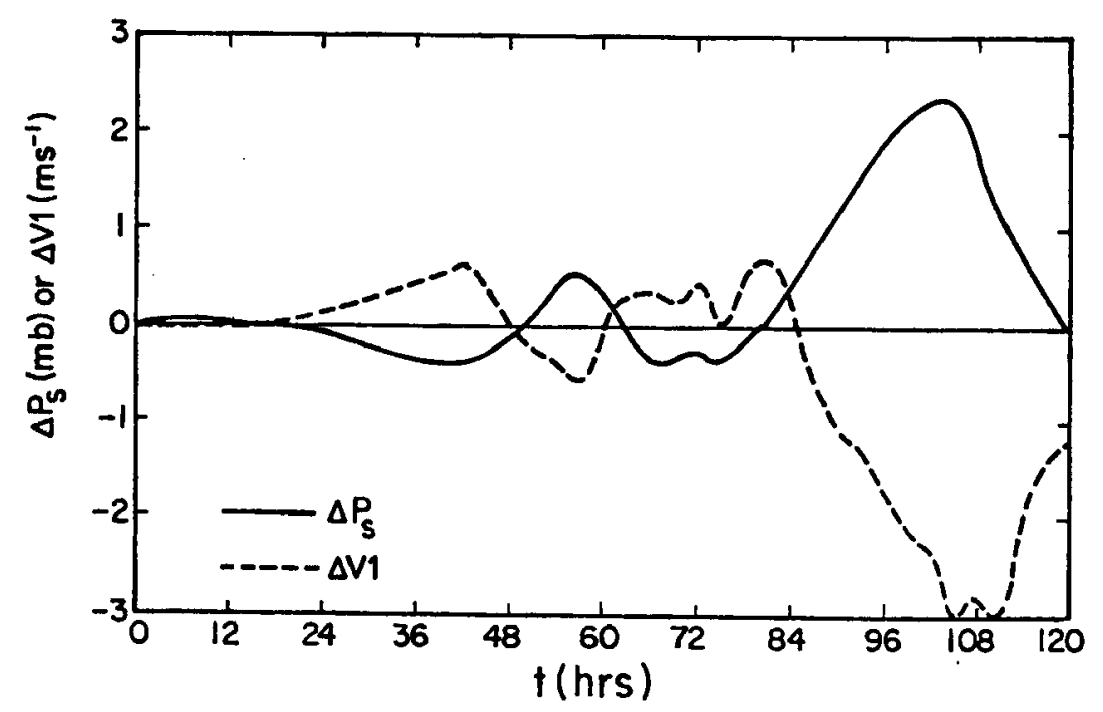

Figure 31. The east-west difference $\Delta x$ and north-south difference $\Delta y$ in the positions of the tropical cyclones in experiments $5 \mathrm{C}$ and $4 C(a)$ and the difference in the surface pressure deviations and maximum layer 1 wind speeds (b). A positive $\Delta x$ or $\Delta y$ indicates that the storm in $5 C$ was farther to the east or north and a positive $\Delta \mathrm{P}_{\mathrm{S}}$ or $\Delta \mathrm{V}$ l indicates that the storm in $5 \mathrm{C}$ had a higher surface pressure or wind speed. 
upper level trough had almost no influence on the position of the storm. After 24 hours, the storm is deflected towards the south as the trough axis moves to the east of the storm center. Since the storm in experiment $5 \mathrm{C}$ is further to the south, it moves more rapidly towards the west since the zonal wind is stronger towards the south. After about 60 hours, the storm in experiment $5 C$ begins to move back towards the $y$ position of the storm in experiment $4 C$ and by 75 hours is further to the north. The storm in experiment $5 C$ remains north of the storm in experiment $4 C$ until about 116 hours when it again is further to the south. It is interesting that the $y$ position of the storm in experiment $5 C$ appears to oscillate around the $y$ position of the storm in experiment $4 \mathrm{C}$ after 72 hours when the trough is far to the east. This indicates that the track of the vortex appears to be stable. In experiment $5 C$, the storm was deflected from its track by the upper level trough, but returned to its original north-south position after the trough had passed. It also appears that the storm "overshot" its original position so that its track oscillates around the track of the storm without the upper level trough.

The north-south oscillation of the storm track induced by the upper layer trough is somewhat different than the classical trochoidial oscillation described by Kuo (1969) and Jones (1977a). The trochoidial oscillation appears to be caused by the asymmetries in the frictional forces which result from a vortex moving through a uniform current, and typically has a period of less than about 20 hours. In Fig. 31 a it can be seen that $\Delta y$ has a period of almost 90 hours so that it is probably related to a different process. In Fig. 4 it can be seen that none of the normal modes of the model have a period near 90 hours. This 
indicates that this oscillation is probably related to a nonlinear interaction of the vortex with the large scale environment. Further study is necessary to better explain this result.

Although the upper layer trough in experiment $5 \mathrm{C}$ had a large effect on the structure of the outflow layer and some effect on the storm track, it had only a small effect on the storm intensity. Fig. 31b shows the difference between the minimum surface pressure deviations and the maximum layer 1 wind speeds for experiments $5 \mathrm{C}$ and $4 \mathrm{C}$. A positive $\Delta V 1$ or negative $\Delta P_{S}$ indicates that the storm in experiment $5 C$ had a 1 arger maximum wind speed or lower surface pressure. In this figure it can be seen that the minimum surface pressure deviation and the maximum layer 1 wind speed are very well correlated so that either of these parameters gives a measure of the difference in the intensity of the two storms. Between about 12 and 48 hours it can be seen that the storm in experiment $5 C$ is slightly more intense when it is affected by the upper level trough. After this time the storm in experiment $5 \mathrm{C}$ is slightly less intense until about 60 hours when it again becomes slightly more intense than the storm in experiment 4C. Until 84 hours, however, the difference in the maximum layer 1 wind speed is less than $0.7 \mathrm{~ms}^{-1}$ and the minimum surface deviations are within $0.6 \mathrm{mb}$ for the two experiments. This indicates that the effect of the upper level trough on the intensification rate is quite small. The only significant difference between the two simulations occurred between about 84 and 120 hours when the storm in experiment $4 \mathrm{C}$ became about $2.5 \mathrm{mb}$ deeper and had a maximum wind about $3 \mathrm{~ms}^{-1}$ stronger than the storm in experiment $5 \mathrm{C}$. Since the only difference between these two simulations was the presence of the upper level trough, this difference in intensity must be related 
to this feature, although the storm was far from the trough by this time. There is some indication that this difference might be related to the difference in the storm tracks since $\Delta P_{S}$ and $\Delta V 1$ are fairly well correlated with $\Delta y$ (with about a 4-5 hour time lag) during this time.

Observations of the influence of upper level troughs on tropical cyclone intensification rates have been presented by several authors. For example, Lewis and Jorgensen (1978) have presented an example of how a baroclinic wave in the upper levels caused the rapid decay of a tropical cyclone. These authors concluded that the vertical shear as well as the upper level convergence suppressed the convection which then caused the rapid decay of the storm. In their case, however, the trough axis passed directly over the tropical cyclone. In the current simulation the trough passed to the north of the storm so that the inner cyclonic region of the storm was not strongly influenced. In addition to this, the diabatic term $Q$ in the model is largely dependent on the vertical velocity at the top of the boundary layer. For this reason, the effect of convergence and subsidence in the upper layer on the cumulus convection may be underestimated with the current cumulus parameterization scheme. This indicates that it may be necessary to use a more complicated parameterization scheme to better simulate this interaction.

In contrast to the above results, Sadler (1976) has presented several examples of storms which intensified rapidly as they interacted with upper level troughs. In most of the cases presented by Sadler, the developing tropical cyclones typically had outflow jets towards the equator until they interacted with a tropical upper-tropospheric trough. After this time, the storms developed a second outflow jet towards the 
pole and intensified rapidly. This is somewhat similar to the results presented in Fig. 30 which indicated that the upper level trough changed the direction of the outflow channel. In the current simulation, however, the storm lost its outflow channel to the south when the outflow channel to the north formed, which may explain why the intensity of the storm was not affected. The results presented by Sadler appear to be somewhat similar to the results from experiment $5 B 2$ which did intensify rapidly when two outflow channels formed.

The above discussion indicates that upper level troughs can either increase or decrease the intensification rates of tropical cyclones. The effect probably depends on the position of the storm relative to the trough, and also possibly on the vertical structure of the trough. In the results presented by Sadler, the trough was restricted to the upper troposphere, while in the case presented by Lewis and Jorgensen, the trough was a stronger baroclinic disturbance which extended through a much deeper layer. Due to the crude vertical structure of the model the results presented here are probably more representative of the influence of a trough which extends through a deep layer. 


\section{CHAPTER 6}

TROPICAL CYCLONE MOTION

Dynamical models have been used operationally to predict tropical cyclone tracks since the development of the SANBAR model by Sanders and Burpee (1968). The errors which occur in track prediction models can result from inadequate model physics, lack of initial data and numerical approximations. The original SANBAR model used the simple twodimensional nondivergent barotropic vorticity equation which was initialized with the rotational part of a vertically averaged wind field. More recently, primitive equation models have been developed for tropical cyclone track prediction which have ranged in complexity from a three-level model with specified heating (Harrison, 1973) to a 10-level model with moving nested grids (Hovermale and Livezey, 1977). When primitive equation models are used for track forecasts, the initialization problem becomes more difficult than for the case of balanced models. In addition to the lack of observations of the large scale environment, it is difficult to specify initial mass and wind fields which are consistent with each other so that spurious gravity wave noise is not generated. This problem is even more difficult if the model contains diabatic heat sources since these will be quite large near the tropical cyclone center and will excite large amplitude gravity waves if the model is not initialized carefully. Much progress has been made in this area since the introduction of nonlinear normal mode initialization procedures by Machenhauer (1977) and Baer and Tribbia 
(1977). Since this time these techniques have been used extensively in global prediction problems (e.g. Daley, 1979; Temperton and Williamson, 1979). In this chapter the application of nonlinear normal mode initialization to the tropical cyclone track prediction problem is discussed.

Although more complicated models have been developed, the SANBAR model still remains competitive with the newer models and also with statistical techniques (Goldenberg, 1982). One reason for this is probably the lack of good initial data in the region of a tropical cyclone. It seems possible that the addition of complicated model physics which can not be initialized correctly might not add to the accuracy of a model forecast. The success of the SANBAR model also indicates that tropical cyclone motion is largely governed by vorticity advection. This will be verified in section 6.2 by comparing primitive equation model track forecasts to nondivergent barotropic model forecasts.

In most operational tropical cyclone track models, the large scale fields are analyzed and a symmetric vortex is added which represents the tropical cyclone circulation. In some cases, the size of the vortex is close to the grid spacing of the model so that the details of the vortex circulation are considered to be unresolvable (Sanders et al., 1975). For this reason, the form of the added symmetric vortex is the same for each forecast. When coarse resolution is used, computational dispersion which occurs in finite difference models can be severe at times. The interface between nested grids also can generate numerical noise. In order to control this numerical noise, spatial filters or horizontal diffusion terms are sometimes added. In section 6.3 the effect of the 
vortex size, horizontal resolution of the model and the addition of horizontal diffusion on a tropical cyclone track are investigated using the nondivergent barotropic model.

\subsection{The Effect of Nonlinear Normal Mode Initialization on A Tropical Cyclone Track Forecast}

In section (3.5), the nonlinear normal mode initialization procedure introduced by Machenhauer (1977) was described. In this section, the effect of this initialization procedure on a tropical cyclone track forecast is investigated. For this purpose, experiment $4 \mathrm{C}$ which considered the development of a tropical cyclone in a nonresting basic state on the $\beta-p l a n e$ is used as a control. The simulation in experiment $4 C$ is stopped after 48 hours and the dependent variables are used as initial conditions for the initialization experiments. It is then possible to determine the effect of the initialization by comparing the new simulation to the previous results.

In the nonlinear normal mode initialization procedure, the amplitudes of the Rossby waves and the inertial oscillations associated with the constant depth boundary layer are not changed, while the gravity wave amplitudes are diagnosed as described in section 3.5. Since the boundary layer modes are not changed, the boundary layer inflow is only slightly changed by the initialization procedure. This indicates that the diabatic heat source will still be active after the initialization procedure is applied. In the real atmosphere, however, it is doubtful that this boundary layer inflow could be measured accurately, especially in the region of a tropical cyclone. This is a weakness of the current model which stems from the assumption of the constant depth boundary layer. As discussed previously, this assumption 
changes the normal modes of the model. If the depth of the boundary layer were allowed to vary, the two boundary layer modes would be replaced by an additional Rossby mode and two more gravity modes. If it were necessary to relax this assumption, however, the arguments for using the incompressible fluid system would probably be less valid. Temperton and Williamson (1979) have shown that in a fully stratified model, Machenhauer's initialization scheme is capable of producing boundary layer inflow. Thus, although the boundary layer inflow in the current model is associated with the slow modes, the results are probably representative of what would occur in a more general model where the boundary layer inflow is associated with the fast modes.

Since the initial data to be used in this experiment comes from a previous simulation, the $\Lambda_{0}$ field is known exactly at the initial time. In a simulation which used real data, this would not be the case since the initial moisture distribution would not be known. In order to keep the experiment as simple as possible, the $\Lambda_{0}$ field was not changed in the initialization procedure.

As described in section 3.5 , the diagnostic relation for the gravity wave amplitudes is solved iteratively. Fig. 32 shows the amount of energy in the external and internal gravity waves (calculated as described in section 4.1) after each iteration. In this figure the $i$ on the $x$ axis indicates the amount of energy in these modes before the initialization procedure is applied. This figure shows that the iteration converges quite rapidly. After one iteration, the energies of the internal gravity and external gravity modes are at about $92.7 \%$ and 99.5\% of their final values. Leith (1980) has shown that on the $f$ plane, the first pass through Machenhauer's initialization scheme is 


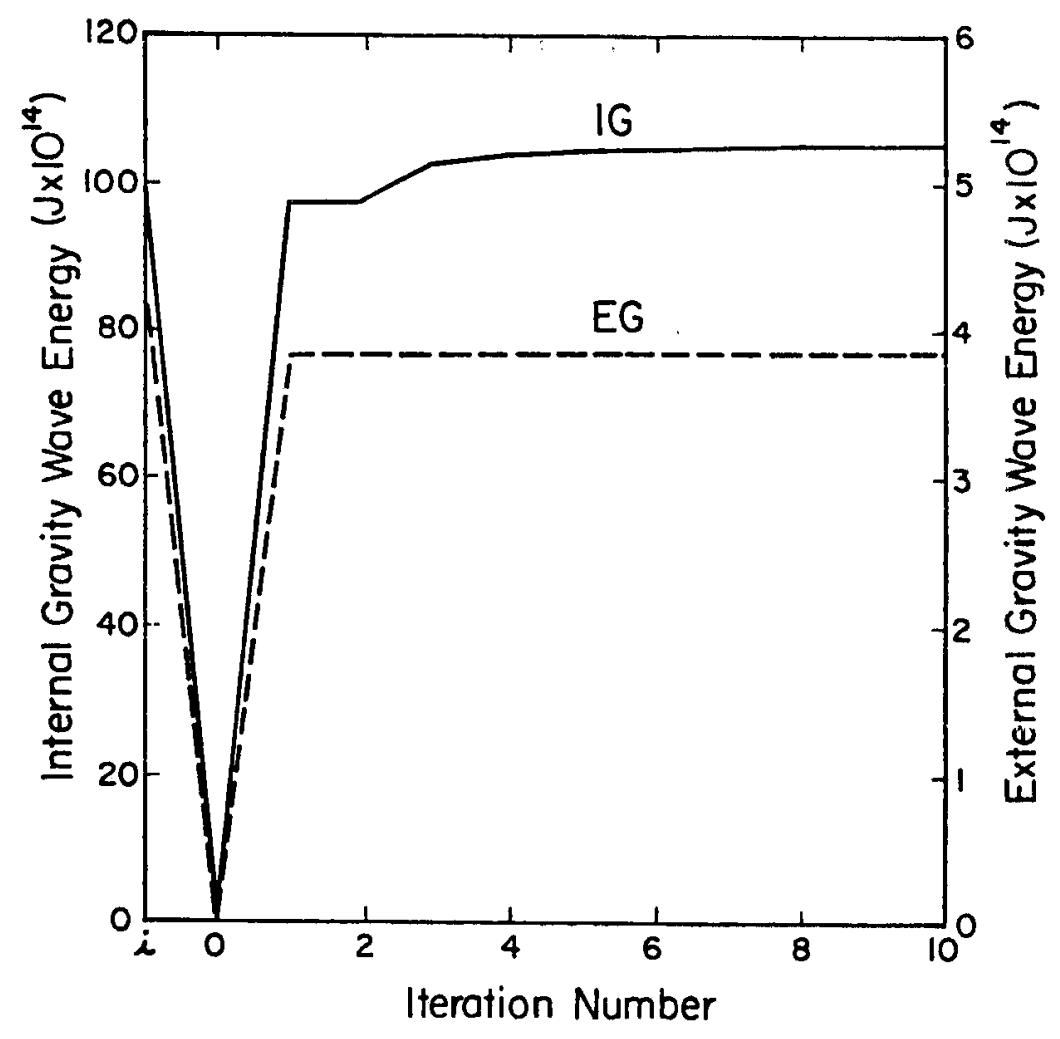

Figure 32. The energy of the internal gravity waves (IG) or external gravity waves (EG) after each iteration of nonlinear normal mode initialization applied to experiment $4 \mathrm{C}$ at 48 hours. The point $i$ on the $x$-axis indicates the energy in the modes before the initialization is applied. 
analogous to applying quasi-geostrophic theory. The fast mode part of the solution which is diagnosed in this case is then probably fairly close to the ageostrophic motion which would be diagnosed using quasigeostrophic theory, except near the center of the vortex where the quasi-geostrophic theory breaks down.

In addition to the nonlinear normal mode initialization, two other initialization procedures were applied. The first of these was a linear normal mode initialization procedure which simply sets the amplitudes of the fast modes to zero at the initial time. The second initialization procedure is based on the nonlinear balance equation. For this case it is assumed that the divergence and the time tendency of the divergence are initially zero. Thus, the velocity potential for each layer is set to zero and the mass field is calculated from the streamfunction using the nonlinear balance equation (4.4). For consistency it must also be assumed that the layer 0 and 1 streamfunctions are the same since the pressure gradient forces are the same for these two layers.

The initialization scheme which uses the nonlinear balance equation to diagnose the mass field from the streamfunction can be generalized to include initial vertical motion. In the nonlinear balanced model, the vertical velocity is diagnosed from an w-equation, analagous to the $\omega$ equation used in quasi-geostrophic theory. Due to the extreme complexity of the wequation in the nonlinear balanced model, however, the complete w-equation is rarely used in practice. For this reason, the simplier approach of using a nondivergent initial wind field is used here. The method used here has been used for the initialization of operational track prediction models (e.g. Harrison, 1973). 
Fig. 33 shows the time evolution of the external and internal gravity wave energy for each of the initialization procedures described above. The solid line in this figure shows the gravity wave energy for the control experiment $4 \mathrm{C}$. In this figure it can be seen that the linear initialization procedure causes a large amount of spurious gravity wave energy to be excited, even though the initial gravity wave amplitudes were set to zero (at $t=48$ hours). This is because the diabatic forcing and the nonlinear interaction of the Rossby modes rapidly produce gravity waves when the model integration begins. This is similar to the results presented by williamson (1976) which showed that linear normal mode initialization was not capable of suppressing gravity wave oscillations in a shallow water equation model.

In constrast to the linear normal mode initialization, the nonlinear initialization did not produce much spurious gravity wave energy. The energy of both the internal and external gravity waves remained very close to the gravity wave energy in experiment $4 C$ which was not initialized at $t=48$ hours. This indicates that the nonlinear normal mode initialization procedure suppressed the artificial generation of gravity wave energy during the 72 hour simulation.

The initialization procedure based on the nonlinear balance equation did not generate as much gravity wave energy as the linear initialization, but did introduce some errors in the gravity wave amplitudes. As can be seen in Fig. 33, this initialization procedure reduced the internal and external gravity wave energy by $56 \%$ and $19 \%$ respectively when applied at 48 hours. After this time the internal gravity wave energy rapidly increases and after 9 hours (at 57 hours) has exceeded the internal gravity wave energy in the control experiment. 

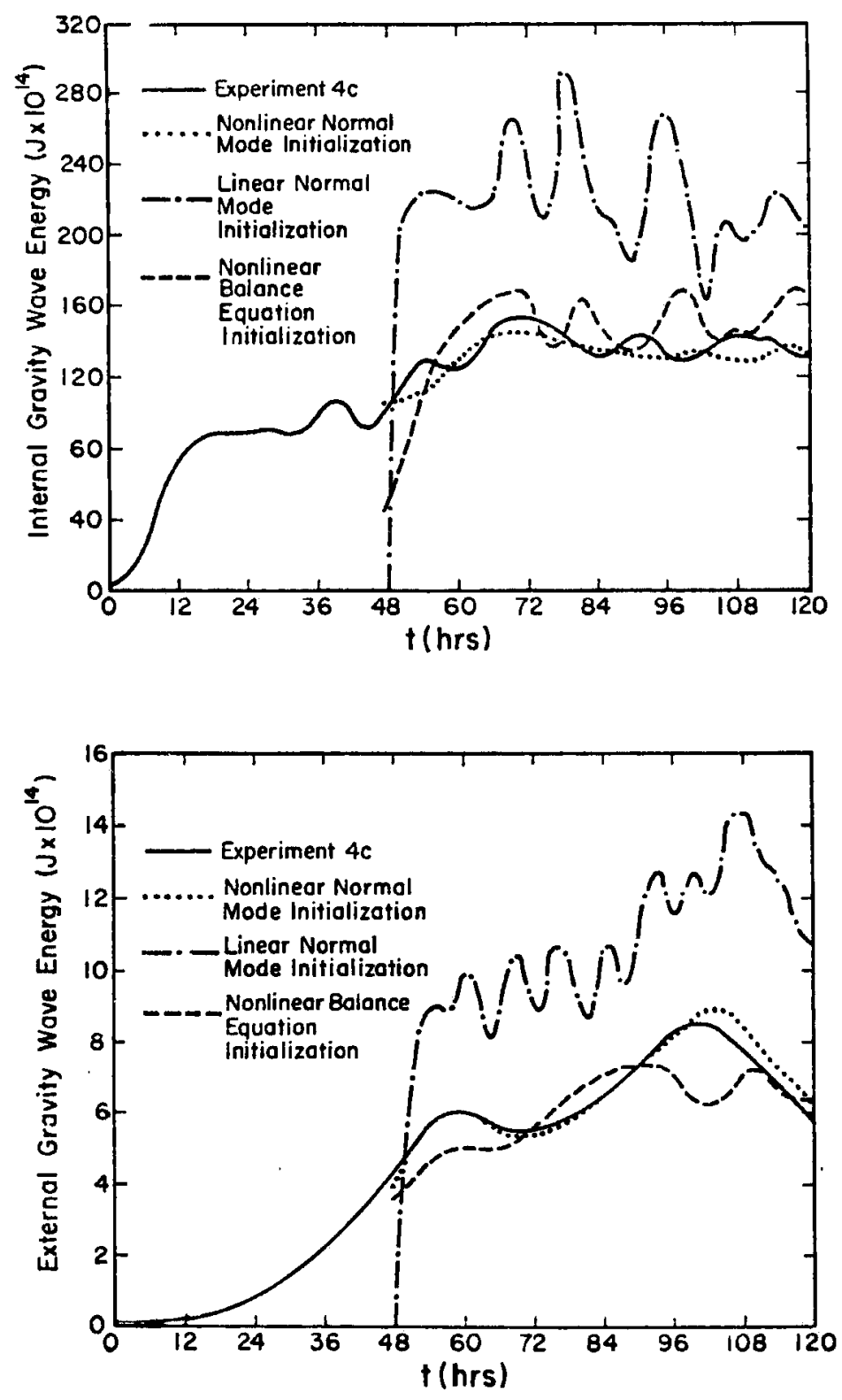

Figure 33. The time evolution of the internal and external gravity wave energy for experiment $4 C$ and after three initialization procedures were applied at 48 hours. 
This rapid increase in the internal gravity wave energy is probably related to the diabatic heat source. Since the initialization procedure assumes that the divergence is zero, the vertical velocity at the top of the boundary layer is zero so that the diabatic term $Q$ is zero. Once the integration begins, the boundary layer inflow rapidly redevelops and $Q$ returns to a value close to what it was before the initialization was applied. As discussed previously, greater amounts of gravity wave energy will be excited by a heat source which varies rapidly in time. Thus, the assumption of a nondivergent initial wind field results in a diabatic heat source which changes rapidly in time as the divergence field increases since the diabatic heat source is parameterized in terms of the boundary layer vertical velocity. This then causes the energy of the internal gravity waves to increase rapidly as the model integration begins.

After about 24 hours (by 72 hours in Fig. 33), the model appears to have adjusted to the nonlinear balance initialization and the energy of both the external and internal gravity waves remain fairly close to the corresponding energies in the control experiment. In terms of the gravity wave energy produced it then appears that nonlinear normal mode initialization gave the best results while the nonlinear balance initialization introduced slightly larger errors and the linear balance initialization excited much larger amounts of spurious gravity wave energy.

Although the amount of gravity wave energy in a model simulation has an effect on the solution, it is probably not a primary concern of forecasters who use operational tropical cyclone track models. Fig. 34 shows the track errors which are introduced by each of the 

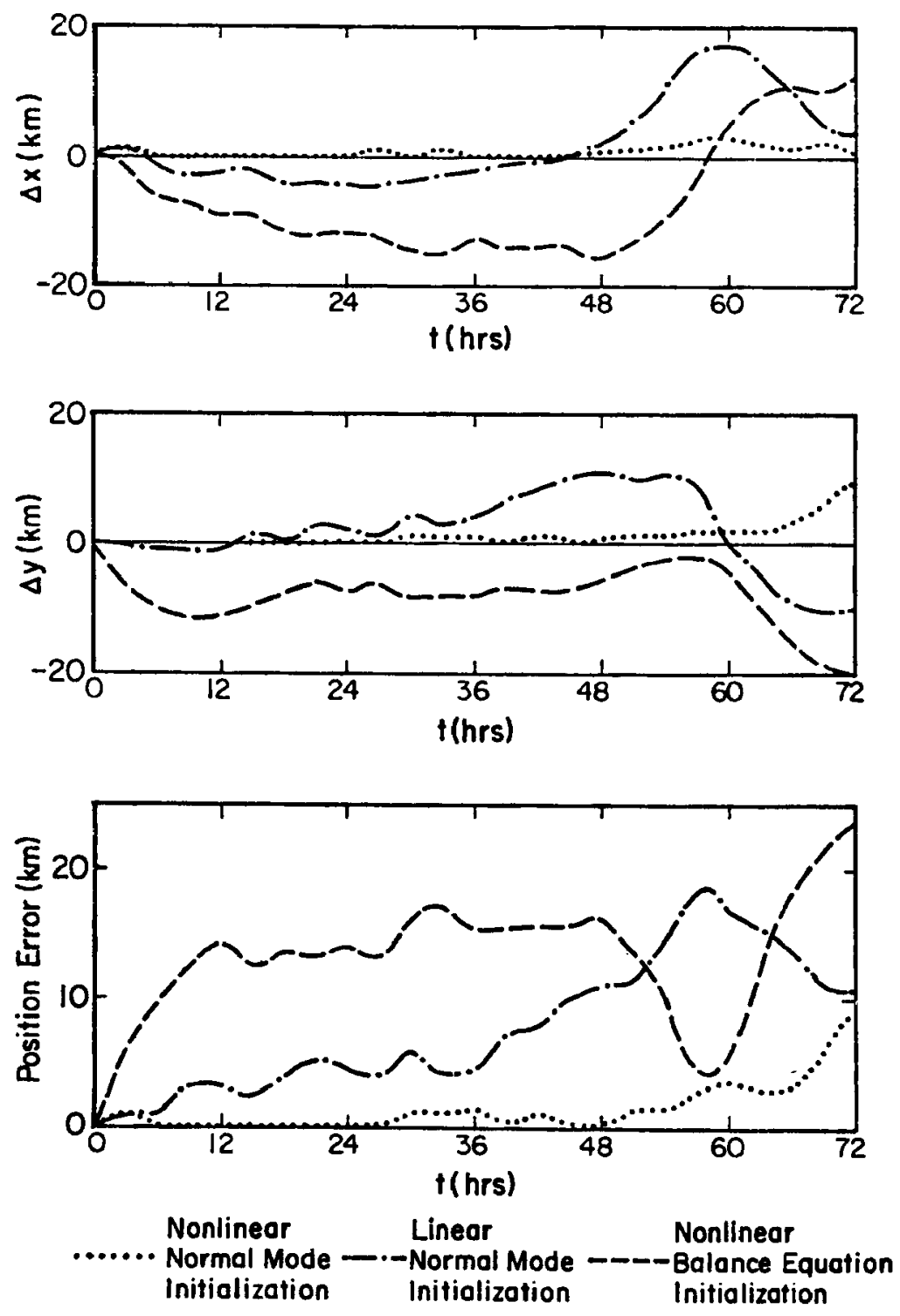

Figure 34. The east-west $(\Delta x)$, north-south $(\Delta y)$ and total position error introduced by three initialization procedures applied to experiment $4 \mathrm{C}$ at 48 hours. 
initialization procedures described above. These errors were calculated by comparing the tracks of the tropical cyclones in the initialization experiments to the track in the control experiment. In this figure a positive $\Delta x$ or $\Delta y$ indicates that the initialized storm was east or north of the storm in the control experiment and the position error is the straight line distance between the two storms. This figure shows that in the first 48 hours, the nonlinear balance initialization procedure introduces the largest position errors while the nonlinear normal mode initialization results in the smallest errors. These errors appear to be related to the assumption of a nondivergent initial wind field for the balance equation initialization. Anthes and Hoke (1975) have shown that the inclusion of divergence in a barotropic model causes a tropical cyclone to track more towards the north. This also appears to be the case in the initialization experiments since after the divergence was set to zero in the balance equation initialization procedure, the storm is further to the south than the control storm ( $\Delta y$ becomes negative in Fig. 34). Since this storm is further to the south, it also moves more rapidly towards the west since the storm is in a mean easterly flow which increases towards the south at the initialtzation time. For the nonlinear normal mode initialization procedure, the initial wind field is divergent so that these errors do not occur, as can be seen in Fig. 34.

When the linear norma 7 mode initialization procedure is applied, the gravity wave amplitudes are set to zero and the divergent part of the wind in the main fluid layers is reduced. For this case, however, the divergent part of the wind in the boundary layer is not affected since this is associated with the boundary layer modes which are 
considered slow in the current model formulation. This indicates that the diabatic forcing was still active after this initialization procedure was applied. Thus, although the divergent wind in the main fluid layers was reduced, it very rapidly increases after the simulation begins due to the diabatic term so that the track errors are not as large as for the balance initialization where both the divergent part of the wind and the diabatic term are set to zero.

In addition to errors in the tropical cyclone track, the initialization procedures also introduced errors in the storm intensity. Fig. 35 shows the time evolution of the errors in the minimum surface pressure and maximum layer 1 wind speed for each of the initialization procedures. These errors were calculated using the control experiment as a reference where a negative $\Delta P_{S}$ or positive $\Delta V 1$ indicates that the initialized storm had a lower minimum surface pressure or larger maximum wind speed. This figure shows that the nonlinear normal mode initialization procedure resulted in the smallest errors in the intensity of the storm both in terms of surface pressure and wind speed. For the nonlinear balance equation initialization, the storm was less intense than the storm in the control experiment for the first 27 hours after the initialization procedure was applied, and a1so between about 39 hours and 63 hours. The initial difference in the storm intensity was probably caused by the effect of this initialization procedure on the diabatic forcing term as discussed previously. This indicates that it may be advantageous to initialize a model with a nonzero diabatic forcing.

Fig. 35 also shows that the linear normal mode initialization procedure has a large effect on the minimum surface pressure of the 

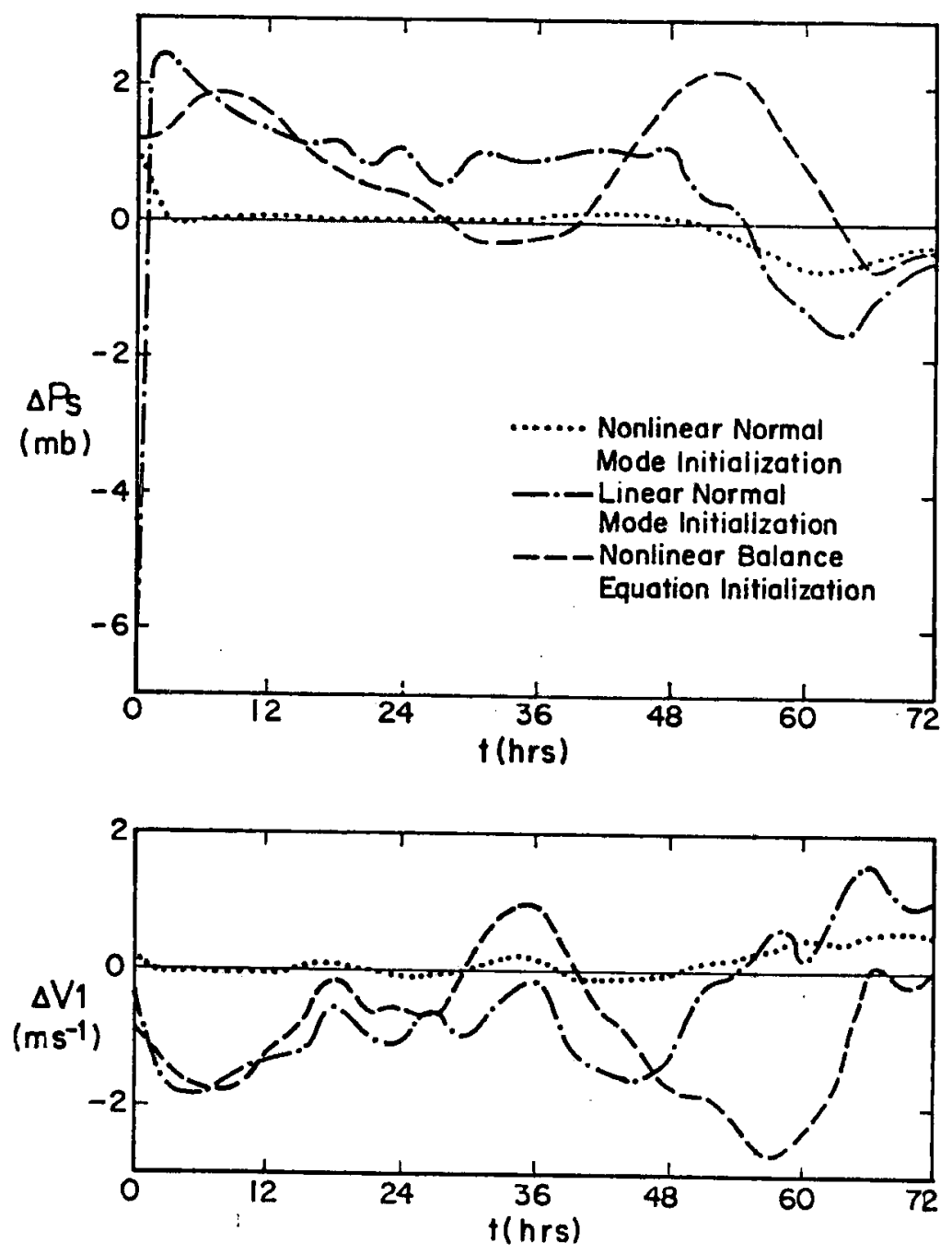

Figure 35. The errors in the minimum surface pressure and maximum layer 1 wind speed introduced by three initialization procedures applied to experiment $4 \mathrm{C}$ at 48 hours. 
storm. After this procedure is applied, the minimum surface pressure is $6.2 \mathrm{mb}$ lower than in the control experiment. This is because near the storm center, the geostrophic approximation is not very accurate (at the initialization time the maximum wind speed is about $30 \mathrm{~ms}^{-1}$ at a radius of $85 \mathrm{~km}$ ), while the Rossby modes in the model are near geostrophic balance since they are based on the linearized equations. Thus, a vortex which is in approximate gradient balance can not be represented by Rossby modes alone, but rather projects onto both gravity and Rossby modes. The linear initialization procedure then causes some distortion near the center of the vortex when the gravity modes are set to zero. As soon as the time integration begins, the surface pressure increases very rapidly which indicates that the vortex is adjusting back towards an approximate gradient balance. This indicates that the linear normal mode initialization procedure is not appropriate for the case when the wind field is very far from geostrophic balance.

The results shown in Figs. 33-35 indicate that the nonlinear normal mode initialization produces the smallest track and intensity errors, and prevents the excitation of spurious gravity wave energy. The largest position error which was introduced by any of the initialization schemes, however, was only about $23 \mathrm{~km}$ after 72 hours, as can be seen in Fig. 34. In contrast to this, the official 72 hour forecasts for storms in the Atlantic basin have an average position error of about $600 \mathrm{~km}$ (Neumann and Pelissier, 1981). Since the official forecast is based on numerical track predictions as well as several other techniques, the small gain in accuracy from the normal mode initialization procedure might be considered negligible compared to the size of the current forecast errors. In the comparisons between the initialization 
procedures, the initial data was obtained from a previous model simulation so that the slow modes for the normal mode procedures and the streamfunction for the balance equation procedure were known exactly. In a simulation using real data, this would not be the case due to poor data coverage so that the errors using both initialization procedures would be much larger. Assuming that the results presented here also applied to the case when realistic initial data was used, the nonlinear normal mode initialization could lead to a reduction in track forecast errors. Further studies using realistic initial data are required, however, to better answer this question.

\subsection{Tropical Cyclone Motion In The Nondivergent Barotropic Model}

The relative success of the SANBAR model introduced by Sanders and Burpee (1968) indicates that the prediction of a tropical cyclone track is somewhat easier than the prediction of intensity changes. The SANBAR model is governed by the equation for the conservation of absolute vorticity which in cartesian coordinates can be written as

$$
\frac{\partial \zeta}{\partial t}+\frac{\partial}{\partial x}(u \zeta)+\frac{\partial}{\partial y}(v \zeta)+\beta v=\lambda \nabla^{2} \zeta
$$

In the above equation the second two terms from the left are the advection of the relative vorticity by the rotational part of the wind and the third term represents the advection of the earth's vorticity where the conventional mid-latitude $\beta$-plane approximation has been made. The term on the right side is a horizontal diffusion term which is sometimes added to crudely simulate atmospheric diffusion and has also been used as a numerical filter. In (6.1) it is assumed that the wind 
field is nondivergent so the horizontal wind components $u$ and $v$ and the relative vorticity $\zeta$ can be expressed in terms of a streamfunction $\psi$ as follows:

$$
\left.\begin{array}{l}
u=-\frac{\partial \psi}{\partial y}, \quad v=\frac{\partial \psi}{\partial x}, \\
\zeta=\frac{\partial v}{\partial x}-\frac{\partial u}{\partial y}=\nabla^{2} \psi .
\end{array}\right\}
$$

In this section and section 6.3 , examples of the motion of a vortex motion governed by $(6.1)-(6.2)$ will be presented. These equations were solved on a doubly-periodic domain using the Fourier-Galerkin method described in chapter 3 for the primitive equation model. Unless otherwise indicated, all of the simulations were run on a 3600 by 3600 $\mathrm{km}$ domain with 36 Fourier modes in each direction using a three minute time step.

As a simple test of the nondivergent barotropic model, a symmetric vortex was added to a zonal flow and the model was integrated for 72 hours. The form of the vortex is given by

$$
V=\frac{2 V_{m}\left(r / r_{m}\right)}{1+\left(r / r_{m}\right)^{2}}
$$

where the maximum tangential wind $v_{m}$ occurs at the radius $r_{m}$. The vorticity profile for the above vortex is given by

$$
\zeta=\frac{4 V_{m}}{r_{m}}\left[1+\left(r / r_{m}\right)^{2}\right]^{-2}
$$

The vortex profile given by (6.3) was chosen since the corresponding vorticity profile is always positive and the tangential wind profile is 
similar to solid body rotation inside the radius of maximum wind. The zonal wind profile used is the same as that which was used in experiment 4C which is given by

$$
u=U \sin \frac{2 \pi y}{L_{y}}
$$

with $U$ equal to $-7.5 \mathrm{~ms}^{-1}$. This zonal wind profile has easterlies in the southern half of the domain and westerlies in the northern half. The vortex given by (6.3) with $v_{m}=30 \mathrm{~ms}^{-1}$ and $r_{m}=80 \mathrm{~km}$ was centered at $x=2200 \mathrm{~km}$ and $y=1400 \mathrm{~km}$ and the model was run without diffusion $(\lambda=0)$. In the remaining part of this chapter, this simulation will be referred to as experiment $6 A$.

Fig. 36 shows the wind field at $t=0$ and $t=72$ hours for the simulation described above, where only a portion of the domain is displayed. Also shown in this figure are the 24 hourly positions of the streamfunction minimum associated with the vortex. In this figure it can be seen that the vortex track is similar to the track of the tropical cyclone in experiment $4 \mathrm{C}$ which is shown in Fig. 16. The motion of the vortex in the nondivergent barotropic model appears to be a combination of the effect of the mean flow and the $\beta$-effect. Initially the track of the vortex is towards the west which is a result of the mean flow. The $\beta$-term in $(6.1)$ also initially causes the vortex to move towards the west since $\beta v$ is acting to decrease the vorticity to the east of the vortex and increase the vorticity to the west of the vortex. As the integration continues, however, the vortex begins to turn towards the north. This turn towards the north can be explained by considering the effect of the advection of the earth's vorticity on the mean flow. Since $\beta V$ is acting to reduce the vorticity to the east of the vortex, it 

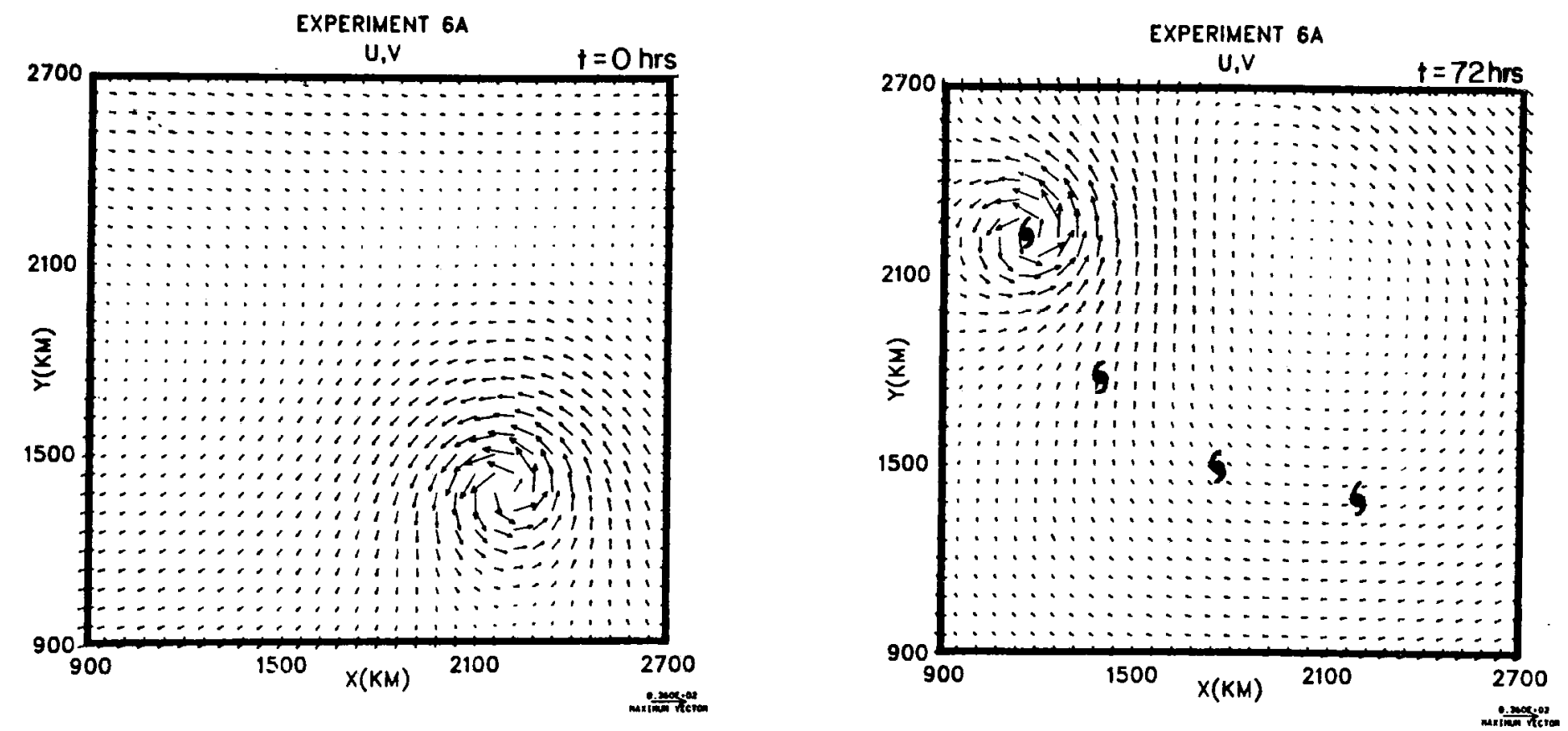

Figure 36. The wind field at 0 and 72 hours for experiment $6 A$. The 24 hourly positions of the streamfunction minimum associated with the vortex are indicated by the synoptic symbol for a hurricane. 
is tending to induce anticyclonic motion, while it is increasing the vorticity to the west of the vortex and thus inducing cyclonic motion. The induced anticyclonic motion to the east and cyclonic motion to the west both result in a northward flow through the center of the vortex which causes the vortex to move more towards the north.

The motion of a vortex in the nondivergent barotropic model is caused by the advection of vorticity. For a vortex similar to the lower levels of a tropical cyclone, the relative vorticity can be two orders of magnitude larger than the earth's vorticity near the vortex center (e.g. Hawkins, 1968). Fig. 37 shows the relative vorticity field for experiment $6 \mathrm{~A}$ at $\mathrm{t}=0$ and $\mathrm{t}=72$ hours. In this figure, the relative vorticity is normalized by the Coriolis parameter $f_{0}$ evaluated at $20^{\circ} \mathrm{N}$ and the contour interval is three nondimensional units. Fig. 37 shows that the relative vorticity of the vortex is a sharp isolated peak in an otherwise fairly uniform field. This figure also shows that after 72 hours, the numerical approximation scheme has allowed the vortex to move without much distortion of the relative vorticity maximum. This indicates that a spectral model would probably give more accurate results when applied to tropical cyclone track prediction than a finite difference model which would distort the sharp peak in the vorticity field. A finite difference model would distort the vorticity maximum because finite difference methods slow down the short wavelength part of the solution so that artificial dispersion is introduced into the solution. In a spectral model this problem is greatly reduced as can be seen in Fig. 37.

The track of the vortex in experiment $6 \mathrm{~A}$ shown in Fig. 36 is similar to the track of the storm in experiment $4 \mathrm{C}$ which was predicted 

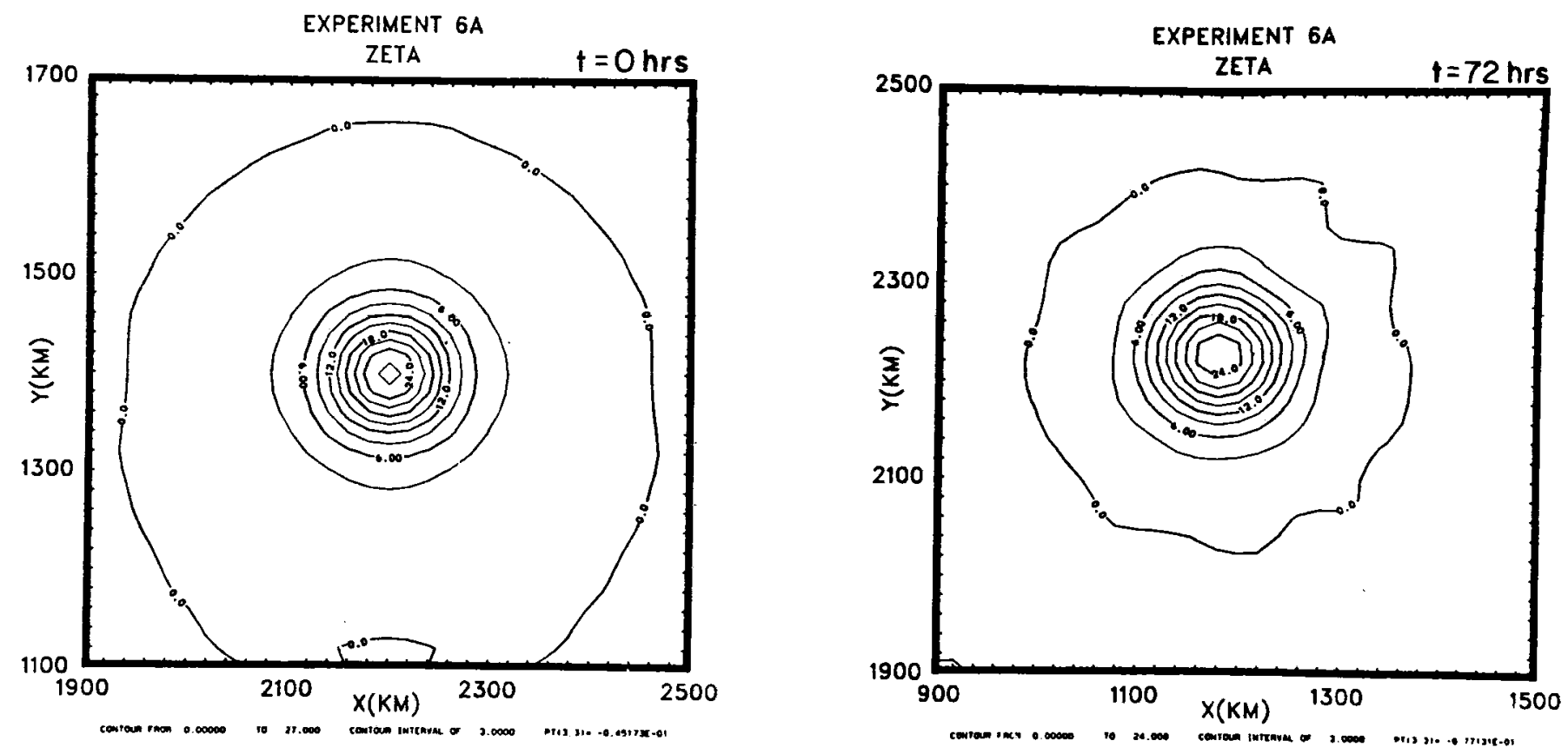

Figure 37. The relative vorticity field associated with the vortex in experiment $6 \mathrm{~A}$ at 0 and 72 hours. The vorticity is normalized by the Coriolis parameter $f_{0}$ and the contour interval is 3 nondimensional units. 
by the primitive equation model. This suggests that the prediction of the track of a tropical cyclone is somewhat easier than the prediction of intensity changes. In order to better assess the ability of the nondivergent barotropic model to predict storm tracks, results from the primitive equation model were used as initial data for the barotropic model. The layer 1 streamfunction from experiment $4 C$ at $t=0,30$ and 60 hours was used to initialize the barotropic model and the resulting tracks of the streamfunction minimum are shown in Fig. 38a. Although there are errors in the resulting tracks, the barotropic model successfully predicts the recurvature for each case.

Fig. 38b shows the time evolution of the position error for each of the barotropic model forecasts. The largest error occurs at $t=96$ hours for the simulation which was initialized using the layer 1 streamfunction from experiment $4 C$ at $t=0$. This result is not surprising since the maximum layer 1 wind speed increased from about $13 \mathrm{~ms}^{-1}$ to 36 $\mathrm{ms}^{-1}$ in the first 96 hours in experiment $4 C$, while the intensity of the vortex in the barotropic model remained approximately constant. This indicates that the intensification does affect the track of the storm. For the barotropic model simulations, the errors are reduced when the model was initialized at the later times. For the simulations initialized at 30 and 60 hours, the position errors remained less than $150 \mathrm{~km}$ which indicates that the barotropic model can predict the track of the tropical cyclone in the primitive equation model in some cases. The simulation which was initialized at 60 hours had position errors which remained less than $100 \mathrm{~km}$. These errors are fairly small when compared to average position errors of $400 \mathrm{~km}$ and $600 \mathrm{~km}$ at 48 hours and 72 hours respectively for operational forecasts (Neumann and Pelissier, 

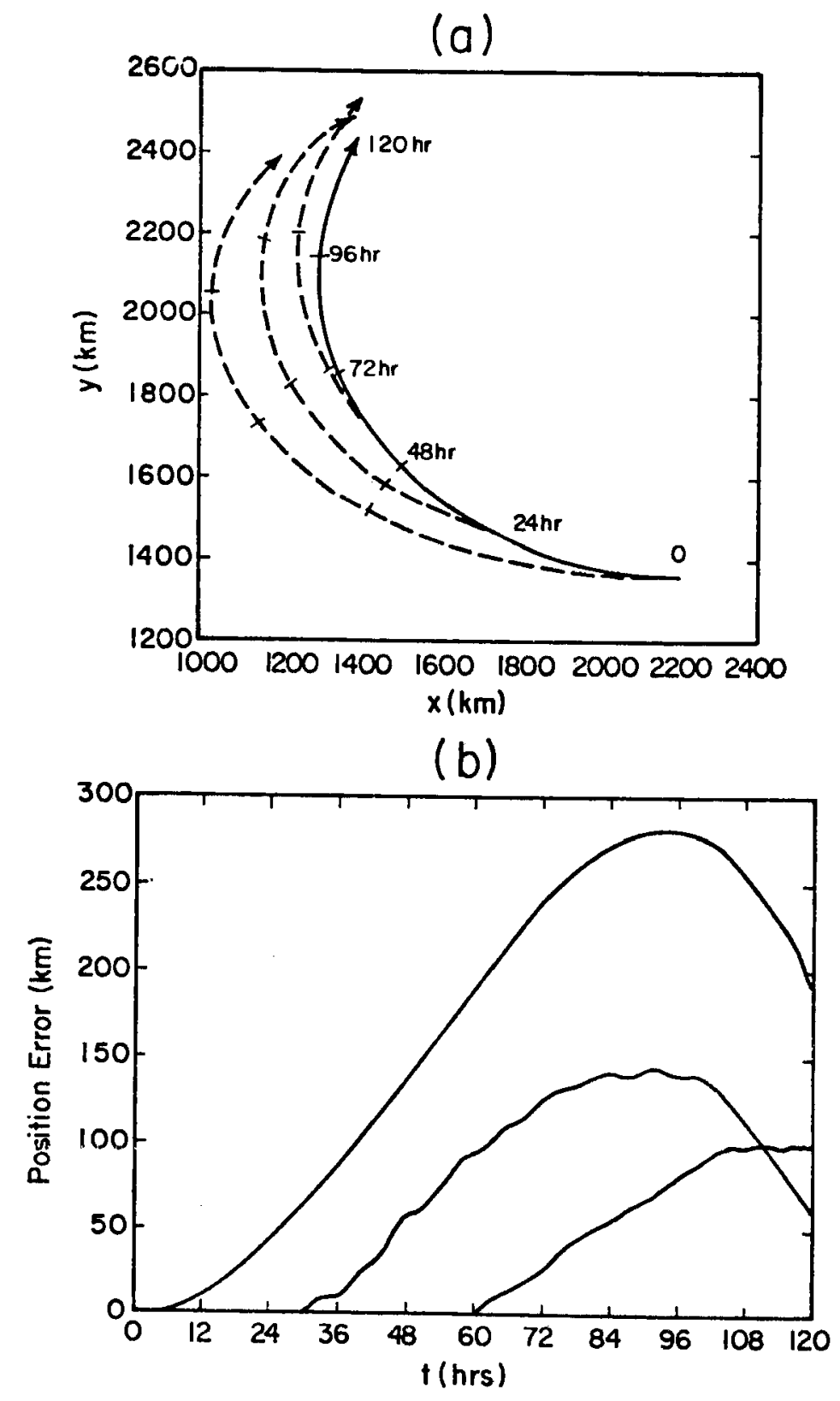

Figure 38. The track of the streamfunction minimum in experiment $4 \mathrm{C}$ (solid) and the corresponding tracks predicted with the nondivergent barotropic model initialized with the layer 1 streamfunction at 0,30 and 60 hours (dashed). Also shown is the time evolution of the position errors introduced by the use of the barotropic model. 
1981). After about 60 hours the tropical cyclone in experiment $4 C$ was not intensifying rapidly, as can be seen in Fig. 16, so that the barotropic model should be expected to give better results when initialized at this time.

The above results indicate that the barotropic model is capable of predicting the track of the tropical cyclone in the primitive equation model, except during periods of rapid intensification. Although a more complicated model would be capable of simulating intensity changes, it is doubtful that the initial conditions could be estimated well enough so that the model would correctly forecast intensity changes. Since the intensification affects the storm track, the inclusion of improperly initialized model physics could lead to larger track errors. This implies that for operational track forecasts, numerical models with simplified physics are probably appropriate.

\subsection{The Effect of Spectral Truncation, Horizontal Diffusion And Vortex Size on Nondivergent Barotropic Model Track Forecasts}

The barotropic model simulations presented in the previous section were all run with 36 Fourier modes in each direction on a $3600 \mathrm{~km}$ square domain. The smallest wave in the model then has a wavelength of about $100 \mathrm{~km}$ which could be resolved in a grid point model with a grid spacing of $50 \mathrm{~km}$. The grid spacing used in operational models varies from about $150 \mathrm{~km}$ for the SANBAR model (Sanders et al., 1975) to about $60 \mathrm{~km}$ on the smallest grid of the moveable fine mesh model (Hovermale and Livezey, 1977). In order to get an idea of the effect of horizontal resolution on a track forecast, experiment $6 \mathrm{~A}$ was run with spectral truncations of $6,12,24,36$ and 48 Fourier modes and the results for each of the 

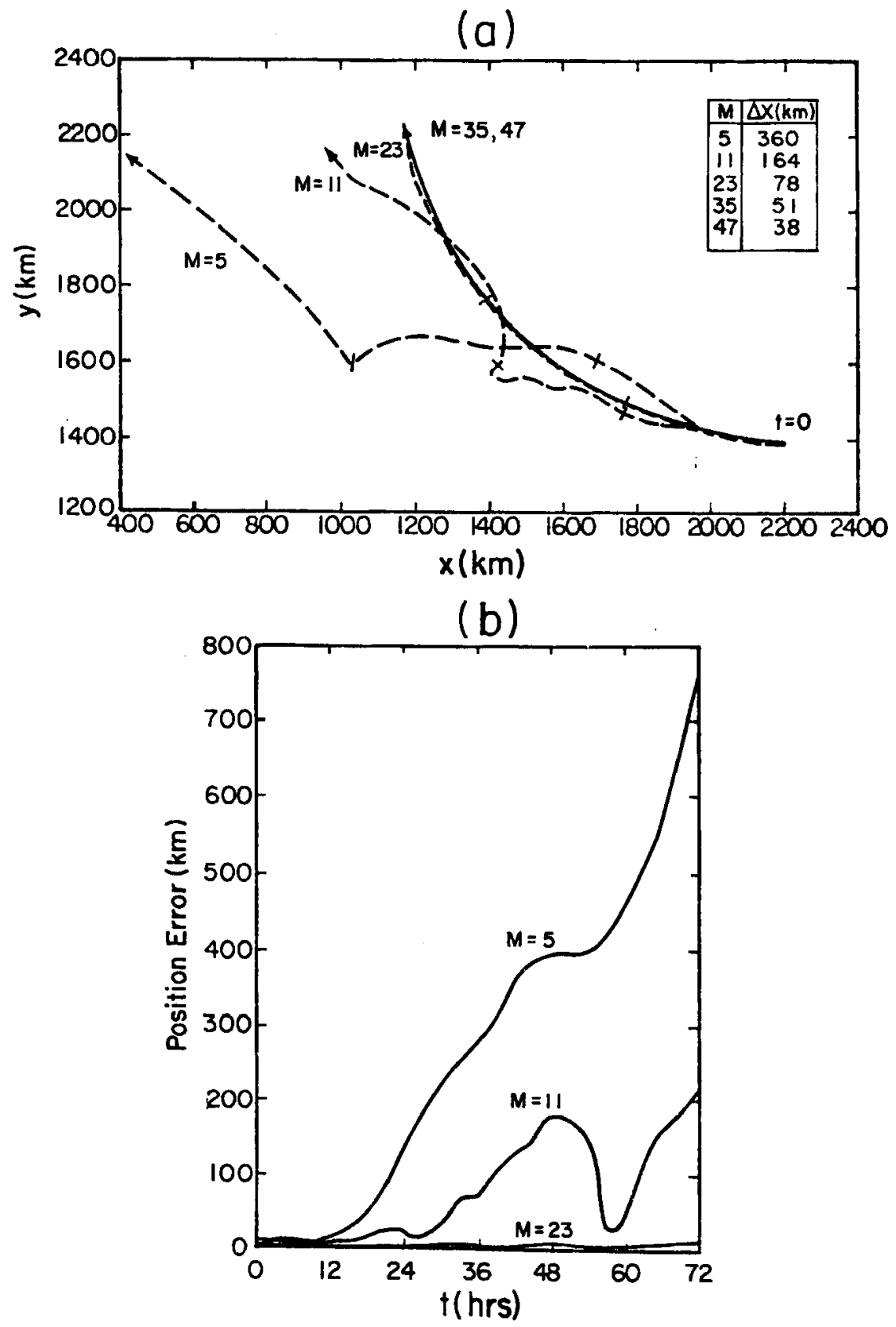

Figure 39. The track of the streamfunction minimum in experiment $6 \mathrm{~A}$ when the model is run with various spectral truncations (a), and the associated position errors (b) using the $M=47$ run as a reference. The upper right corner of (a) shows the equivalent grid spacing of the $2 \Delta x$ wave for each spectral truncation. 
simulations were compared. Although the changing of the resolution in a spectral model has a different effect than for a grid point model due to the differences in the convergence rates, these results should give some idea of the minimum amount of resolution necessary in a track forecast.

Figure 39a shows the track of the streamfunction minimum associated with the vortex in experiment $6 \mathrm{~A}$ for each of the spectral truncations and Fig. 39b shows the position errors compared to the $M=47$ simulation. This figure shows that the position errors rapidly decrease as the spectral truncation increases. The error for the $M=35$ case is not shown in Fig. 39b since the vortex position differed by less than $2 \mathrm{~km}$ from the $M=47$ case during the 72 hour simulation. This rapid reduction in error with increasing resolution is a reflection of the rapid convergence of the spectral method.

The upper right corner of Fig. 39a shows the grid spacing which would be required in a finite difference model to resolve the smallest wave for each of the spectral truncations. For the $M=11$ case the grid spacing required is $164 \mathrm{~km}$ which is close to the resolution of the operational SANBAR model. Since this case has a position error of about $200 \mathrm{~km}$ after 72 hours, it appears that this resolution is inadequate for accurately predicting a tropical cyclone track. Since the phases of the smallest wavelengths in a finite difference model are very inaccurate, the position errors would probably be even larger than the errors presented for the spectral method.

The tracks of the vortex shown in Fig. 39a were determined by locating the position of the streamfunction minimum every three hours. In track forecast models, the vortex position is usually determined by locating either a streamfunction minimum or a vorticity maximum. In 
some cases the positions of the streamfunction minimum and the vorticity maximum start to diverge during a numerical simulation. For the simulations shown in Fig. 39, the position of the streamfunction minimum and vorticity maximum at 72 hours differed by $89 \mathrm{~km}$ for the $M=5$ case and by $9 \mathrm{~km}$ for the $M=47$ case. This indicates that inadequate model resolution may result in a divergence of these two positions.

In all of the simulations shown in Fig. 39 , the basic state wind was represented exactly since it was specified to be proportional to wave number one in the y-direction. The symmetric vortex which was added to the zonal wind, however, was not represented exactly since the Fourier series are truncated. In order to get an idea of how well the vortex was represented for each of the spectral truncations, the azimuthal average of the magnitude of the wind was calculated using a coordinate system centered on the streamfunction minimum using the initial conditions of each simulation. Fig. 40 shows that the vortex is not represented very well for $M=5$. This is not surprising since the smallest wavelength for this case is $720 \mathrm{~km}$ while the vortex has a radius of maximum wind of $80 \mathrm{~km}$. As the spectral truncation increases, the solution converges quite rapidly and the difference between the $M=35$ and $M=47$ solutions were too small to be represented on the graph. The errors in the representation of the vortex shown in this figure result in the position errors shown in Fig. 39. If this experiment was repeated using a finite difference model, the initial wind components would be specified exactly at the grid points for any resolution so it might be argued that the errors in the initial representation would be smaller. Since finite difference models are based on approximating functions by truncated Taylor series, the derivatives of the wind field 


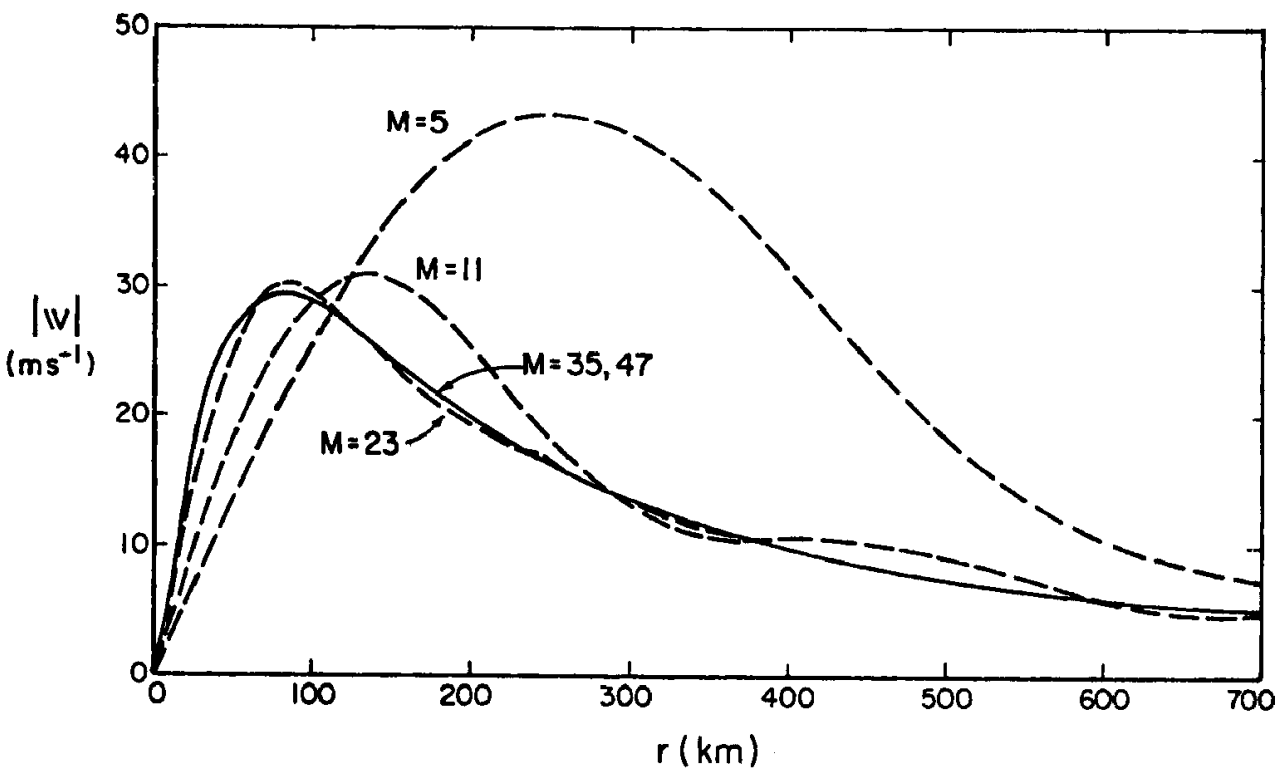

Figure 40 . The representation of the vortex in experiment $6 \mathrm{~A}$ using several spectral truncations. The $M=35$ and $M=47$ curves have converged to the analytic vortex profile to within the resolution of the diagram. 
at the grid points would have initial errors so that this argument probably would not hold.

When dynamical models were first used for operational track prediction after the introduction of the SANBAR model by Sanders and Burpee (1968), it was necessary to use very coarse grid resolution because of computing constraints. One solution to this problem is the use of variable size grids which, as described by Elsberry (1978), is appropriate for the tropical cyclone problem since the gradients in the dependent variables are much larger near a storm than in the large scale environment. Harrison (1973) designed a three-level primitive equation model for tropical cyclone track prediction that has a fine grid which follows the storm center. One problem with using variable mesh grids is that numerical noise is sometimes created at the grid interfaces. In order to overcome this problem, it is often necessary to apply some type of spatial smoother to the dependent variables. For example, Harrison (1973) added diffusion terms of the form $\lambda \nabla^{2}()$ to the momentum equations to filter numerical noise.

In order to determine the effect of diffusion on the track of a tropical cyclone, experiment $6 \mathrm{~A}$ was run with several different values of $\lambda$, where $\lambda$ is the horizontal diffusion coefficient in (6.1). Fig. 4la shows the track of the streamfunction minimum for $\lambda=0,10^{3}, 10^{4}$ and $10^{5}$ $\mathrm{m}^{2} \mathrm{~s}^{-1}$ for the case where the initial condition was the same as for experiment $6 \mathrm{~A}$. Fig. $41 \mathrm{~b}$ shows the position errors as a function of time, where the case without diffusion $(\lambda=0)$ was used as a reference. This figure shows that the track of the vortex is not sensitive to the diffusion coefficient until it exceeds about $10^{4} \mathrm{~m}^{2} \mathrm{~s}^{-1}$. When $\lambda=10^{5}$ 
(a)

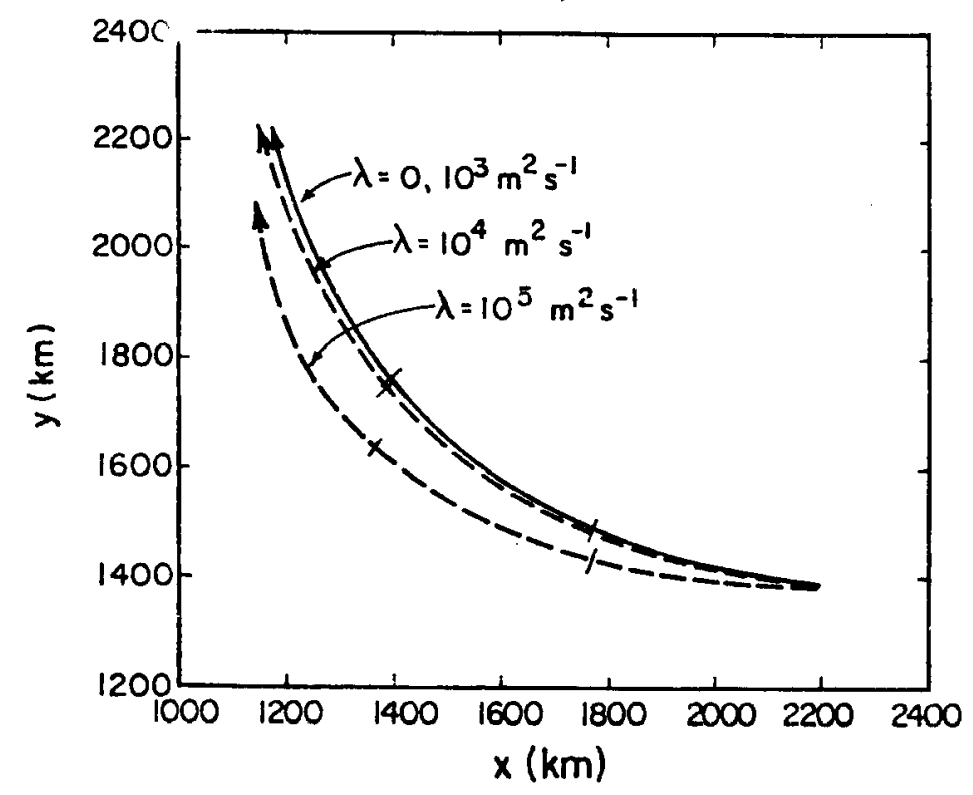

(b)

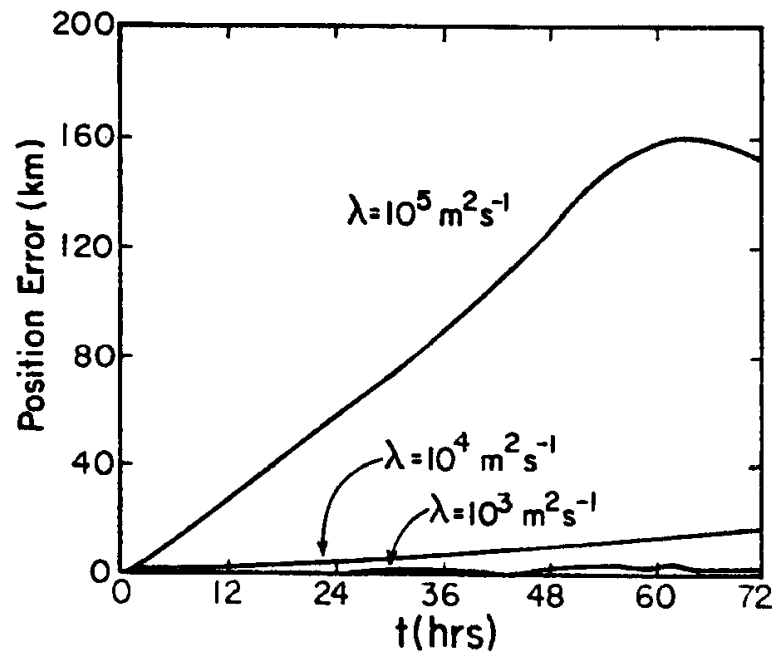

Figure 41. The tracks of the streamfunction minimum in experiment 6 A when second-order linear diffusion is added with various diffusion coefficients (a) and the assosiated position errors using the case without diffusion $(\lambda=0)$ as a reference (b). 
$\mathrm{m}^{2} \mathrm{~s}^{-1}$, the center of the vortex is about $150 \mathrm{~km}$ away from the vortex for the case without diffusion after a 72 hour forecast.

In order to get a better idea of how the diffusion affects the vortex, the azimuthally averaged value of the magnitude of the wind was calculated using a cyclindrical coordinate system centered on the streamfunction minimum associated with the vortex, as shown in Fig. 42. The solid line in this figure is the vortex wind profile at the initial time while the dashed lines are the profiles after 72 hours. This figure shows that the diffusion term acts to reduce the wind speed of the vortex, particularly near the vortex center. Fig. 42 also shows that when $\lambda=10^{5} \mathrm{~m}^{2} \mathrm{~s}^{-1}$, the vortex is almost completely dissipated by 72 hours. It might be noted that in the model by Harrison (1973), horizontal diffusion terms were added with a diffusion coefficient as large as $2.5 \times 10^{5} \mathrm{~m}^{2} \mathrm{~s}^{-1}$. From the results presented here it appears that this value is too large.

In Fig. 42 it can be seen that when $\lambda=10^{4} \mathrm{~m}^{2} \mathrm{~s}^{-1}$ the maximum wind speed associated with the vortex was reduced from about $30 \mathrm{~ms}^{-1}$ to 17 $\mathrm{ms}^{-1}$ and the radius of maximum wind increased from $80 \mathrm{~km}$ to $140 \mathrm{~km}$ during the 72 hour simulation. In Fig. 41, however, it can be seen that the position error for this case was less than $20 \mathrm{~km}$ throughout the simulation. This indicates that the track of a vortex in the nondivergent barotropic model is not sensitive to the wind structure near the vortex center. In order to further investigate this result, experiment $6 \mathrm{~A}$ was run after a symmetric tangential wind perturbation given by

$$
V^{\prime}=V_{m}^{\prime}\left(\frac{r}{r_{m}}\right)^{2} \exp 2\left[1-\left(\frac{r}{r_{m}}\right)\right]
$$




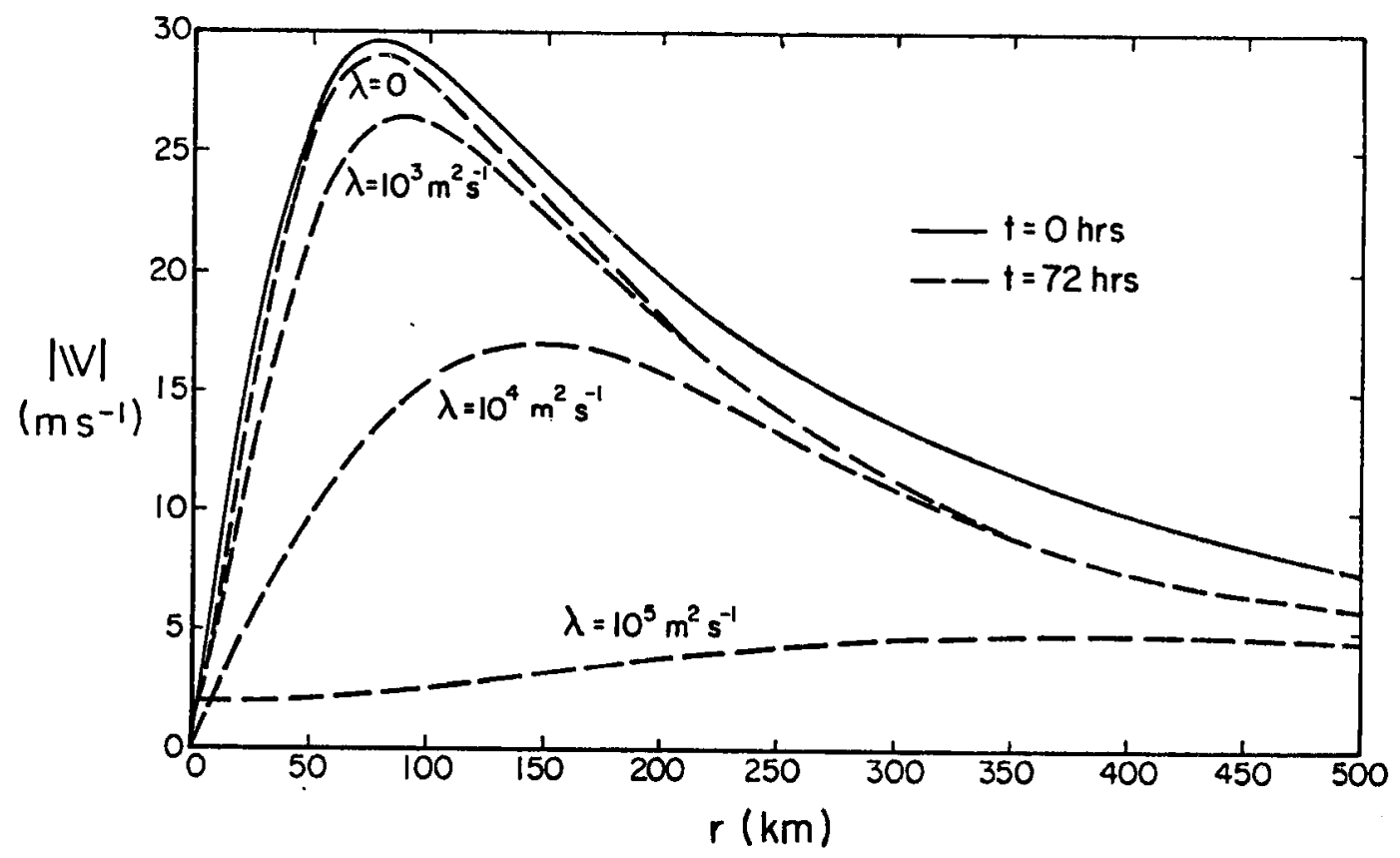

Figure 42. The azimuthally averaged wind speed profiles associated with the vortex in experiment $6 \mathrm{~A}$. The profiles at 72 hours using several diffusion coefficients are the dashed curves and the initial profile is the solid curve. 
was added to the vortex defined by (6.3). The perturbation given by (6.6) with $v_{m}^{\prime}=30 \mathrm{~ms}^{-1}, r_{m}{ }^{\prime}=40 \mathrm{~km}$ and $v_{m}{ }^{\prime}=5 \mathrm{~ms}^{-1}, r_{\mathrm{m}}{ }^{\prime}=300 \mathrm{~km}$ was added to the vortex in experiment $6 \mathrm{~A}$ to test the sensitivitiy of the model to the vortex structure inside and outside the radius of maximum winds. Fig. 43 shows the tangential wind profile for experiment $6 \mathrm{~A}$ and the resulting profiles after the two forms of the perturbation are added. This figure shows that the first perturbation (referred to as experiment $6 \mathrm{~B})$ changes the wind profile inside $150 \mathrm{~km}$ while the second perturbation (6C) changes the profile outside of about $100 \mathrm{~km}$. In order to resolve the vortex structure, experiment $6 \mathrm{~B}$ was run with a spectral truncation of $M=47$, while experiment $6 C$ was run with $M=35$.

Fig. 44 shows the track of the streamfunction minimum for experiments $6 A, 6 B$ and $6 C$ and the position errors for experiments $6 B$ and $6 C$ where experiment $6 \mathrm{~A}$ was used as a reference. This figure shows that the track of the vortex is not very sensitive to the tangential wind profile near the radius of maixmum wind, but is sensitive to the tangential wind at larger radii. After the 72 hour simulation the position error was only about $25 \mathrm{~km}$ for experiment $6 \mathrm{~B}$ even though the maximum tangential wind of the vortex was increased from $30 \mathrm{~ms}^{-1}$ to over $50 \mathrm{~ms}^{-1}$. In contrast to this, the position error after 72 hours in experiment $6 \mathrm{C}$ was about $150 \mathrm{~km}$ even though the tangential wind was increased by a maximum of $5 \mathrm{~ms}^{-1}$ at a radius of $300 \mathrm{~km}$. These results indicate that the inclusion of some information about the size of a tropical cyclone in a model track forecast is probably more useful than including information about the maximum tangential winds.

In summary, the results from the nondivergent barotropic model indicate that except during periods of rapid intensification, this 


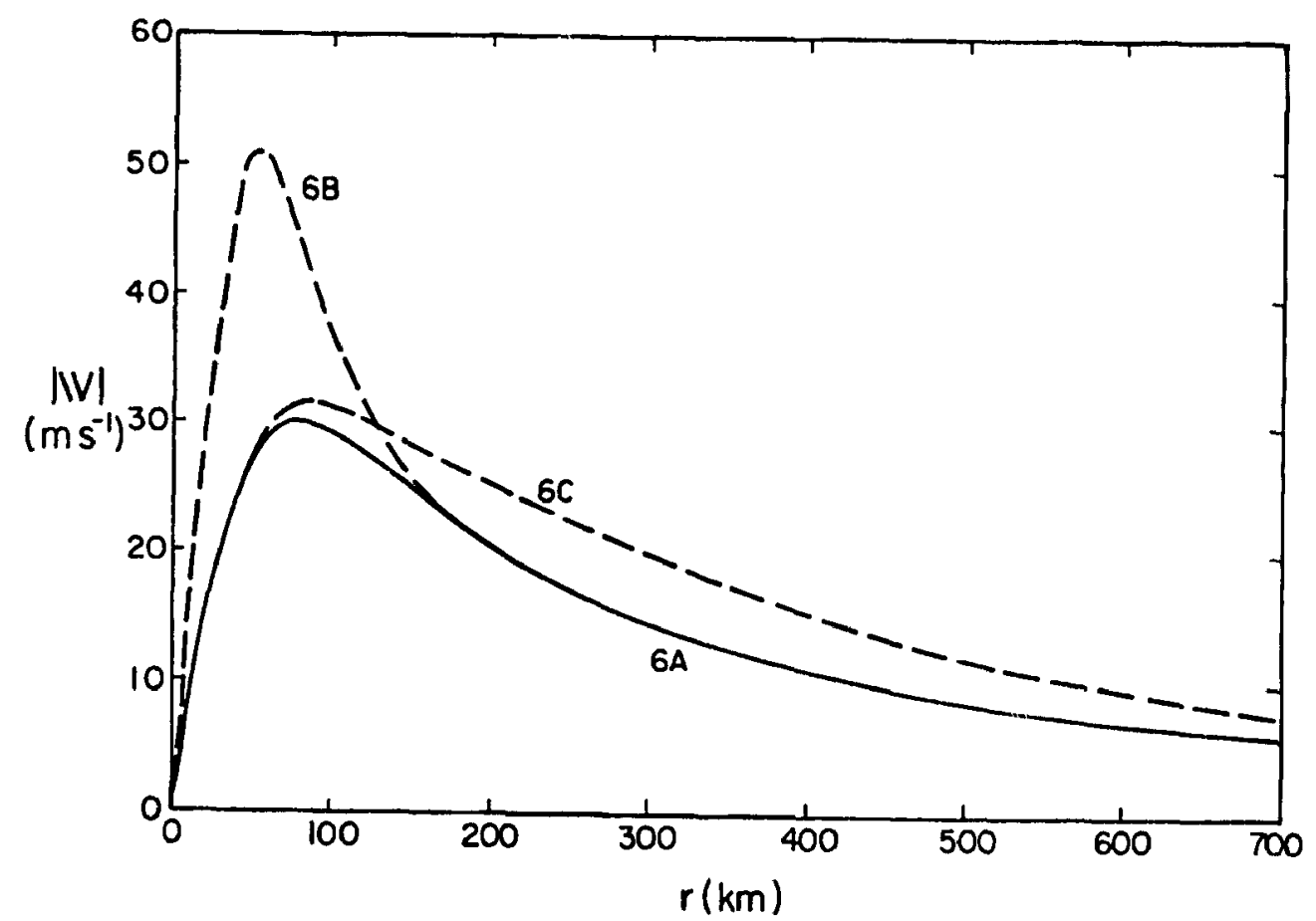

Figure 43. The initial tangential wind profiles of the vortices used in experiments $6 \mathrm{~A}, 6 \mathrm{~B}$ and $6 \mathrm{C}$. 
(a)

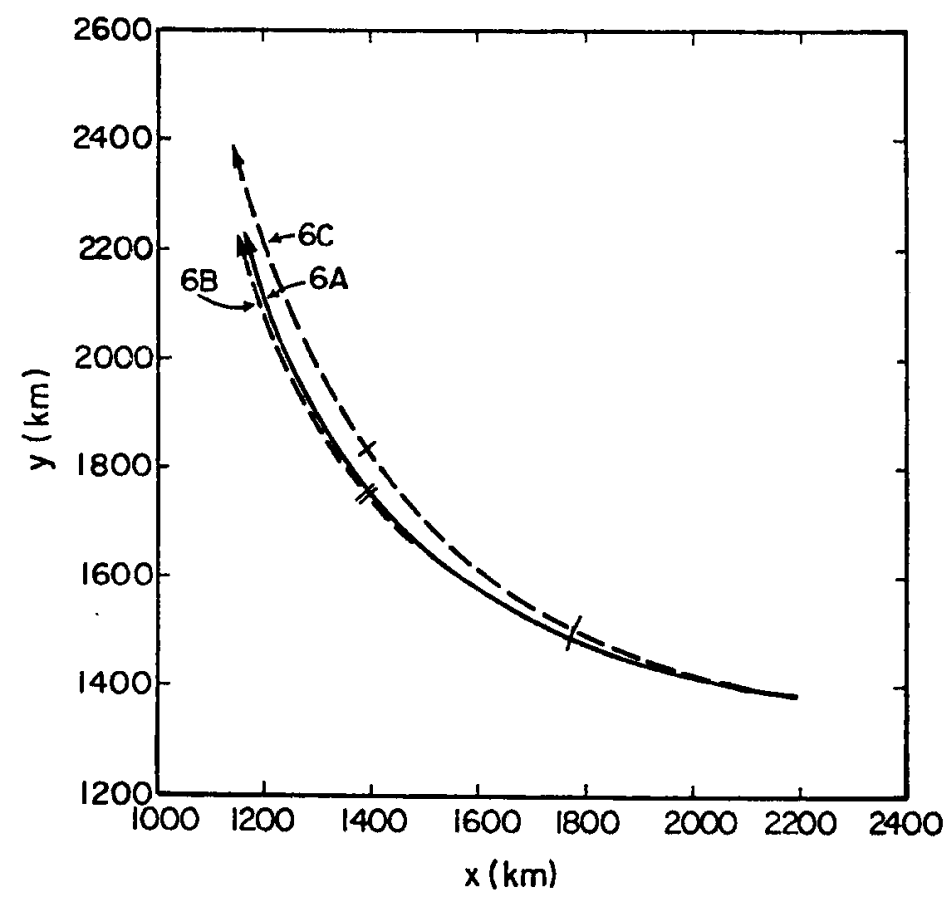

(b)

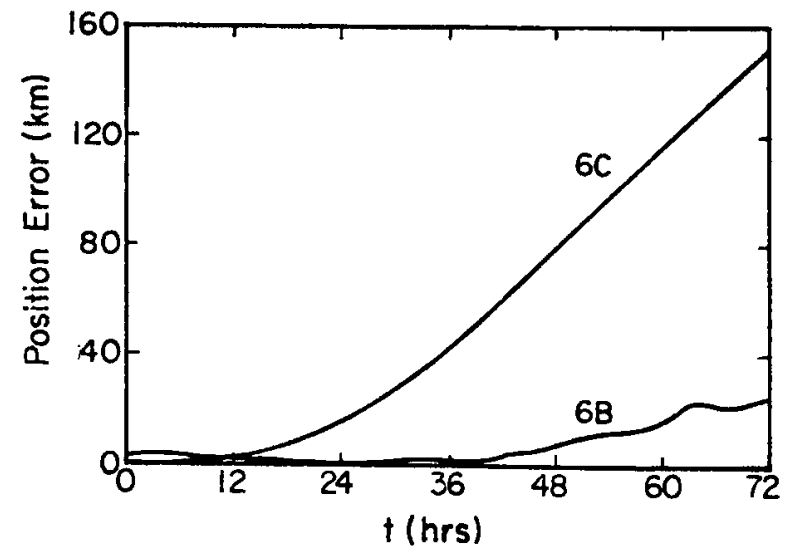

Figure 44. The tracks of the streamfunction minimum in experiments $6 \mathrm{~A}, 6 \mathrm{~B}$ and $6 \mathrm{C}(\mathrm{a})$ and the position errors in experiments $6 B$ and $6 C$ using experiment $6 A$ as a reference (b). 
simple model can predict the track of a tropical cyclone in the primitive equation model. These results also show that the horizontal resolution of the model, large horizcntal diffusion and the structure of the vortex outside the radius of maximum wind all affect the vortex track. The above results imply that it might be possible to improve operational model track forecasts by increasing the model resolution, including some information about the vortex size, and in some cases by making a more careful choice of the diffusion coefficient. 
CHAPTER 7

SUMMARY AND CONCLUSIONS

The three-1ayer hurricane model developed by Ooyama (1969a) has been generalized to three-dimensions and the resulting equations solved on a mid-latitude $\beta-p l$ ane using a spectral method. The derivation of the model equations was presented in chapter 2 and the numerical solution using the Galerkin method with Fourier basis functions was discussed in chapter 3. Ooyama's model was used in this study because it is perhaps the simplest model which can produce a vortex similar to tropical cyclones observed in nature. The solution technique presented here could also be used for more general three-dimensional tropical cyclone models. The major difference between the current model which considers incompressible fluid layers and a fully stratified model is the treatment of the vertical structure. In a fully stratified model it would be necessary to use a finite difference method or perhaps a finite element method (Staniforth and Daley, 1977) in the vertical. Once this was done, the resulting discretized equations could be solved using the method presented in chapter 3. Assuming that the vertical discretization was consistent with the original equations, the separation of the vertical modes would be possible so that the governing equations could be written in terms of the model normal modes. As shown in chapter 3 , this makes it possible to calculate the linear terms of the model exactly and makes the use of the nonlinear normal mode initialization scheme proposed by Machenhauer (1977) straightforward. 
Another advantage to writing the governing equations in terms of the normal modes is that the total solution can be interpreted in terms of a gravity wave part and a Rossby wave part where the amplitudes of each part are known explicitly.

One disadvantage of the Fourier-Galerkin method used here is that it is necessary to assume periodicity in both the east-west and the north-south direction. This assumption limits the way the Coriolis parameter can be treated ir, the model. With periodicity in the northsouth direction, it is inappropriate to use spherical geometry or even an equatorial $\beta-p l a n e$ since the governing equations contain operators which are not periodic for these cases. It is possible, however, to use the mid-latitude $\beta-p l$ ane as was described in chapter 2. Another disadvantage to using a doubly-periodic domain for a tropical cyclone simulation is that the gravity waves which are generated by the convective forcing can not leave the domain. As described in chapter 4, this is not a severe problem in the current model since the time scale of the diabatic forcing is long enough that the amount of gravity wave energy generated is much smaller than might be expected from the linear theory of geostrophic adjustment. The periodic boundary conditions also make it difficult to use real data for initial conditions. This is not a problem in this study since the model was primarily designed as a research tool, but it is doubtful that this method could be used in an operational model.

As mentioned in the introduction and in chapter 3 , more general boundary conditions could be included by using the tau method described by Gottlieb and Orszag (1977). This method of solution is similar to the method used here except that Chebyshev polynomials are used in place 
of the Fourier basis functions. For the tau method extra degrees of freecom are added so that the entire series satisfies the boundary conditions rather than each term of the series as in the Galerkin method. This method requires more computational work, however, since the Chebyshev polynomials oscillate rapidly near the boundaries. This makes it necessary to use semi-implicit time differencing methods in order to avoid the need for a restrictively small time step. When semiimplicit methods are used in the tau method with Chebyshev basis functions, a linear system must be solved at each time step. The linear system which results contains a full matrix so that iterative procedures must be used to make the model efficient. These problems can be overcome as was shown by Haidvogel et al. (1980) who used a method similar to this for a balanced barotropic ocean model. It is left as a topic for future research to apply this spectral technique to a tropical cyclone problem. Assuming that the convergence properties of the Chebyshev polynomials are similar to those of the Fourier components and that the linear system required in the tau method can be solved efficiently, it is feasible that a spectral tropical cyclone model with open boundaries could be developed.

When spectral methods with global basis functions (basis functions which are nonzero over the entire domain) are applied, an increase in the number of degrees of freedom results in an increase in the horizontal resolution over the entire domain. In grid point methods, however, it is possible to increase the resolution in parts of the domain by using variable mesh grids. This method has been used in several tropical cyclone models (e.g. Jones, 1977b; Kitade, 1980) to increase the resolution near the vortex center. The basic idea behind 
using variable mesh grids is to increase the accuracy of the solution with a smaller increase in the number of degrees of freedom than would be required if the resolution was increased over the entire domain. Although it is not possible to vary the resolution over portions of the domain in a spectral model, the advantage of using a spectral method is similar to this. As shown by Gottlieb and Orszag (1977), when an appropriate spectral method is used to solve a differential equation, the approximate solution converges to the true solution exponentially as the number of degrees of freedom increases. In comparison to this, the approximate solution obtained using a finite difference method converges algebraically to the true solution. For example, when second order finite difference schemes are used, the error is proportional to the square of the grid spacing. Thus, the use of a spectral method allows more accurate solutions to be obtained using fewer degrees of freedom than would be required in a conventional grid point method. The results presented here show that the spectral method can be used to solve a tropical cyclone problem. The best numerical method to use probably depends on several factors including desired accuracy, the problem to be studied and available computing time.

In chapter 4, four simulations using the spectral tropical cyclone model developed in chapters 2 and 3 were presented. The first simulation considered an axisymmetric vortex on an f-plane. These results showed that the model is capable of producing a tropical cyclone similar to those which are observed in nature. The results from the axisymmetric case were also similar to the results presented by 0oyama $(1969 a, b)$. The differences between the results from the current model and 0oyama's model appeared to be related to the use of primitive 
equations in the current model and to the differing horizontal resolution. When the model was initialized with an axisymmetric vortex, the tropical cyclone which developed remained axisymmetric, which indicates that the use of periodic boundary conditions was not a major problem. For this simulation the energy of the gravity mode and rotational mode parts of the solution were calculated and it was shown that the gravity mode energy was more than an order of magnitude smaller than the rotational mode energy. This verifies the argument that if the time scale of the heating is long compared to the period of the gravity waves, much less gravity wave energy will be excited than might be expected from an initial value experiment with all the heat added instantaneously. This also explains why the tropical cyclone remained axisymmetric even though the gravity waves which propagated through the boundary reentered the domain on the opposite side.

The second simulation presented in chapter 4 considered an axisymmetric initial vortex on the $\beta-p l a n e$. This simulation showed that the inclusion of $B$ caused the tropical cyclone to move towards the north-northwest at about $2 \mathrm{~ms}^{-1}$ in agreement with several other authors. The inclusion of $\beta$ also slowed the intensification rate of the tropical cyclone and caused the intensification to stop after a shorter amount of time than on the f-plane. The structure of the $\beta-p l a n e$ storm became asymmetric even though the initial condition was axisymmetric. In the lower layers there was a tendency for the vortex to be elongated to the west and to develop sharper geopotential gradients to the east of the storm center. This structure was explained in terms of the westward dispersion of the long Rossby wave part of the solution and the eastward dispersion of the short Rossby wave part. In the upper layer the 
outflow from the storm tended to form a jet towards the southwest of the vortex center rather thar: a symmetric anticyclone which occurred in the simulation on the f-plane. On both the $\beta-p l a n e$ and the f-plane, a small region of cyclonic rotation formed in the upper layer near the storm center due to the upward transport of momentum by the convection (the model included cumulus momentum transport). This cyclonic region was much weaker for the $\beta-p l a n e$ case which appeared to be related to the motion of the storm.

The third simulation considered a basic state wind field in addition to the inclusion of $\beta$. The intensification rate and the large scale storm structure for this case were similar to the previous simulation. For this case, however, the vertical velocity at the top of the boundary layer was also asymmetric with a vertical velocity maximum located in the right front quadrant of the storm in relation to the direction of motion. This feature is similar to the results of Shapiro (1983) who presented steady state solutions of the boundary layer flow under a moving vortex. A feature similar to this has also been observed in nature.

The fourth simulation considered an initial condition which contained a wave-like disturbance without a closed circulation. These results showed that the wave axis developed a southwest to northeast tilt similar to the structure of easterly waves which occur in the eastern Atlantic region, and intensified to tropical storm strength in about 96 hours. Some of the features of this simulation were similar to results from Kurihara and Tuleya (1981) who presented a similar simulation using much more detailed model physics. 
In chapter 5 , the effect of the large scale environment on a tropical cyclone simulation was considered. In order to keep the experiments as simple as possible, a symmetric vortex on the f-plane was added to a horizontally sheared zonal flow. The vortex was positioned in such a way that the zonal wind was antisymmetric with respect to the vortex center. This, along with setting $\beta=0$, caused the vortex to be stationary. The results from these simulations were somewhat surprising. When an anticyclonically sheared zonal wind was included in the lower layers, the vortex intensified more rapidly initially, but leveled off after a shorter amount of time than the case without the zonal wind. The resulting tropical cyclone for this case was also much smaller than the case without the basic state wind. When a cyclonically sheared zonal wind was added, the vortex intensified less rapidly at small radii, and at about the same rate at larger radii. These results showed that the effect of large scale vorticity in the lower layers is somewhat complicated. This is because it has an effect on the inertial stability which can affect the rate at which a diabatic heat source will produce balanced flow (Schubert and Hack, 1982), but also affects the secondary circulation which in turn affects the diabatic heat source in the current model formulation.

When the zonal wind was included in the upper layer, the intensification rate also was affected. In one case, the outflow from the tropical cyclone interacted with the large scale flow which resulted in the formation of two outflow jets, which caused the intensification rate to be greatly increased. When the outflow region was more symmetric, the intensification rate of the model tropical cyclone was reduced. 
In chapter 5 , a more realistic case was also presented which considered the effect of an upper level trough on a tropical cyclone simulation. When a trough in the upper layer passed to the north of the vortex, an outflow channel developed towards the trough. When this occurred, the outflow channel which existed towards the southwest in a control experiment was greatly reduced, and the intensity of the tropical cyclone was only slightly affected.

The results in chapter 5 show that the large scale environment does have an effect on tropica? cyclone intensification and structure. This is probably a partial explanation for the wide variety of tropical cyclones which occur in nature.

The simulations which were presented in chapter 5 represent only a small fraction of experiments which could be performed. The effect of the inertial stability in the lower levels on the efficiency of a diabatic heat source in relation to the effect on the secondary circulation could probably be studied using an axisymmetric model. The results presented in chapter 5 appear to indicate that the increased inertial stability in a cyclonic vortex causes the diabatic heat source to evolve more slowly and occur at a larger radius. This effect acts to slow down the initial intensification rate and appears to be more important than the increase in efficiency of the heating which results from the increased inertial stability. Further study is necessary to verify this result.

In contrast to the effect of the large scale environment in the lower levels, it is probably necessary to use a three-dimensional model to study the upper level effects. The results presented here show that the development of asymmetries in the upper layer has a large effect on 
the intensification of the model tropical cyclone. In the current model, the outflow layer is quite thick due to the simple vertical structure used. The upper layer of the model is about $5 \mathrm{~km}$ thick which corresponds to a pressure interval of about $450 \mathrm{mb}$, while the outflow from a tropical cyclone typically occurs in a thinner layer above about $300 \mathrm{mb}$ (e.g., Frank, 1977). Further studies of the effect of the large scale flow in the upper layers on a tropical cyclone should probabiy be made with a model which has better vertical resolution.

In chapter 6 the effect of nonlinear normal mode initialization on the prediction of a tropical cyclone track was studied. Results from a previous model run were used to initialize the model using nonlinear normal mode initialization, linear normal mode initialization and a procedure based on the nonlinear balance equation. These simulations showed that the nonlinear normal mode initialization resulted in the smallest errors in the track and intensity of the tropical cyclone when compared to the model integration when the initial data was not changed. The nonlinear normal mode procedure was also capable of suppressing the excitation of spurious gravity wave energy.

The results presented in chapter 6 also show that the linear normal mode procedure which sets the gravity wave amplitudes to zero is inappropriate for a tropical cyclone model. Basically this is because the geostrophic approximation is very inaccurate near the center of a tropical cyclone, while the Rossby modes of the model are in approximate geostrophic balance since they are based on linear theory. A vortex in gradient balance will then be projected onto both Rossby and gravity modes. When the gravity modes are set to zero the vortex will be out of gradient balance so that an adjustment process must take place. When 
this procedure was applied, the minimum surface pressure changed by about $9 \mathrm{mb}$ in the first two hours as this adjustment took place.

The above results suggest that nonlinear normal mode initialization could be used in an operational primitive equation track forecast model such as the moveable fine mesh (MFM) model described by Hovermale and Livezey (1977). In the current model, however, the vertical structure and cumulus parmaeterization are relatively simple and the normal modes of the model can be determined analytically. In a grid point model it would be necessary to find the eigenvalues and eigenvectors of a matrix which is of degree $3 M N$ where $M$ and $N$ are the number of grid points in the $x$ and $y$ directions. This would be even more difficult in a limited area model since the normal modes of the model also depend on the boundary conditions. Despite these difficulties, Temperton and Williams on (1979) have successfully applied Machenhauer's initialization scheme in a global grid point model so that perhaps this technique could be applied if the boundary conditions in a limited area model did not prove to be a major problem.

Another problem with nonlinear normal mode initialization which did not occur in the current model is that the scheme does not always converge. Since the vertical structure of the current model was so crude, only the external and first internal modes were represented. As discussed in chapter 3 , there is then a large frequency separation between the Rossby and gravity modes.: When better vertical resolution is used, higher vertical modes can be included, so that this frequency separation is reduced since the high internal modes have smaller gravity wave frequencies. As discussed by Daley (1981), the lack of frequency separation can result in nonconvergence of the initialization procedure. 
The inciusion of moist physics also can result in nonconvergence of the procedure (e.g., Puri and Bourke, 1982). This did not occur in the current model, but might be a problem if a more detailed parameterization scheme were used.

Several examples of tropical cyclone motion using a nondivergent barotropic model were presented in chapter 6 . These results showed that the barotropic model could predict the track of the tropical cyclone in the primitive equation model, except during periods of rapid intensification. Other simple examples were presented which showed that the horizontal resolution of the model, the inclusion of strong diffusion and the vortex wind profile outside the radius of maximum wind all affect the predicted tropical cyclone track. These results have implications for procedures which are used in operational models. Results from the barotropic model also showed that the sharp vorticity peak associated with the vortex was not dispersed by the numerical approximation scheme as the vortex was advected. This indicates that the spectral method could be used to obtain accurate results in a tropical cyclone track prediction model. A prototype model governed by the nondivergent barotropic vorticity equation (similar to the SANBAR model) could be solved using the tau method described previously. For this case the model could be initialized with real data and the track forecasting errors could be compared to the conventional SANBAR model. The results presented here indicate that the spectral model should give improved tropical cyclone track forecasts. 


\section{REFERENCES}

Anthes, R.A., 1982: Tropical cyclones: their evolution structure and effects. Meteor. Monographs, vol. 19, No. 41, AMS, Boston, MA,

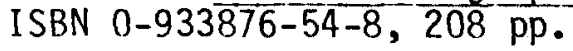

Anthes, R.A. and J.E. Hoke, 1975: The effect of horizontal divergence and latitudinal variation of the Coriolis parameter on the drift of a model hurricane. Mon. Wea. Rev., 103, 757-763.

Baer, F., and J. Tribbia, 1977: On complete filtering of gravity modes through nonlinear initialization. Mon. Wea. Rev., 105, 1536-1539.

Bennett, J.R., 1976: Open boundary conditions for dispersive waves. J. Atmos. Sci., 33, 176-182.

Bjerknes, J., 1938: Saturated-adiabatic ascent through dryadiabatically descending environment. Quart. J. Roy. Meteor. Soc., $\underline{64}, 325-330$.

Bliss, V.L., 1980: Numerical simulation of tropical cyclone genesis. Ph.D. dissertation, Dept. of Atmos. Sci., University of Washington, $268 \mathrm{pp}$.

Bourke, W., 1974: A multi-level spectral model. I. Formulation and hemispheric integrations. Mon. Wea. Rev., 102, 687-701.

Bourke, W., B. McAvaney, K. Puri, and R. Thurling, 1977: Global modeling of atmospheric flows by spectral methods. Methods in Computational Physics, Vol. 17: General Circulation Models of the Atmosphere, Academic Press, $\overline{267-324 . ~}$

Cane, M., and E.S. Sarachik, 1976: Forced baroclinic ocean motions: I. The linear equatorial unbounded case. J. Mar. Res., 34, 629-665.

Challa, M., and R. Pfeffer, 1980: Effects of eddy fluxes of angular momentum on model hurricane development. J. Atmos. Sci., 37, 16031613.

Charney, J.G., and A. Eliassen, 1964: On the growth of the hurricane depression. J. Atmos. Sci., 21, 68-74.

Chen, Lian-shou, 1983: Global view of the upper level flow patterns associated with tropical cyclone intensity change during FGGE. Dept. of Atmos. Sci. Paper (in preparation), Colo. State Univ., Ft. Collins, CO, 80523. 
Colon, J.A., and W.R. Nightingale, 1963: Development of tropical cyclones in relation to circulation patterns at the $200 \mathrm{mb}$ level. Mon. Wea. Rev., 91, 329-336.

Daley, R., 1979: The application of non-linear normal mode initialization to an operational forecast model. Atmos. Ocean, 17, $97-124$.

Daley, R., 1981: Normal mode initialization. Reviews of Geophysics and Space Physics, 19, 450-468.

Eliasen, E., B. Machenhauer and E. Rasmussen, 1970: On a numerical method for integration of the hydrodynamical equations with spectral representation of the horizontal fields. Report No. 2, Kobenhavns Universitet, Institut for teoretisk meteorolgi.

Elsberry, R.L., 1978: Prediction of atmospheric flows on nested girds. Computational Techniques for Interface Problems, vol. 30, Appl. Mech. Div., Amer. Soc. Mech. Eng., 67-86.

Frank, N.L., and G. Clark, 1979: Atlantic tropical systems of 1978. Mon. Wea. Rev., 107, 1035-1041.

Frank, W.M., 1977: The structure and energetics of the tropical cyclone, Paper I: Storm structure. Mon. Wea. Rev., 105, 11191135.

Frank, W.M., and W.M. Gray, 1980: Radius and frequency of $15 \mathrm{~ms}^{-1}$ (30 kt) winds around tropical cyclones. J. Appl. Meteor., 19, 219-223.

Fulton, S.R., and W.H. Schubert, 1980: Geostrophic adjustment in a stratified atmosphere. Dept. of Atmos. Sci. Paper No. 326, Colo. State Univ., Ft. Collins, C0, 80523.

Gazdag, J., 1976: Time-differencing schemes and transform methods. J. Comput. Phys., 20, 196-207.

Goldenberg, S.B., 1982: Recent modifications to the barotropic operational track forecast model (SANBAR). Paper presented at the 14th Technical Conference on Hurricanes and Tropical Meteorology, June 7-11, 1982, San Diego, CA.

Gottlieb, D. and S.A. Orszag, 1977: Numerical Analysis of Spectral Methods: Theory and Applications. Regional conference series in applied mathematics, No. 26.

Gray, W.M., 1979: Hurricanes: Their formation, structure, and likely role in the tropical circulation. Meteorology Over the Tropical Oceans, D.B. Shaw, Ed., Roy. Meteor. Soc., 155-218.

Hack, J.J., and W.H. Schubert, 1981a: The role of convective-scale processes in tropical cyclone development. Dept. of Atmos. Sci. Paper No. 330, Colo. State Univ., Ft. Collins, C0, 206 pp. 
Hack, J. J., and W.H. Schubert, 1981b: Lateral boundary conditions for tropical cyclone models. Mon. Wea. Rev., 109, 1404-1420.

Haidvoge1, D.B., A.R. Robinson, and E.E. Schulman, 1980: The accuracy, efficiency and stability of three numerical models with application to open ocean problems. J. Comput. Phys., 34, 1-53.

Harrison, E.J., 1973: Three-dimensional numerical simulations of tropical storms utilizing nested finite grids. J. Atmos. Sci., 30, 1528-1543.

Hawkins, H.F., and S.M. Imbenbo, 1976: The structure of a smal1, intense hurricane, Inez 1966. Mon. Wea. Rev., 104, 418-442.

Hawkins, H.F., and D.T. Rubsam, 1968: Hurricane Hilda, 1964, 2: Structure and budgets of the hurricane on 0ct. 1, 1964. Mon. Wea. Rev., 96, 617-636.

Herbert, P.J., 1978: Intensification criteria for tropical depressions of the Western Northern Atlantic. Mon. Wea. Rev., 106, 831-840.

Holland, G.J., 1983: Tropical cyclones in the Australian/Southwest Pacific Region. Dept. of Atmos. Sci. Paper No. 363. Colo. State Univ., Ft. Collins, CO 80523.

Hoskins, B.J., and A.J. Simmons, 1975: A multi-layer spectral model and the semi-implicit method. Quart. J. Roy. Met. Soc., 101, 637-655.

Hovermale, J.B., and R.E. Livezey, 1977: Three-year performance characteristics of the NMC hurricane model. Preprints 11th Tech. conf. Hurricanes and Tropical Meteorology, Miami, Amer. Meteor. Soc. , 122-124.

Jones, R.W., 1977a: Vortex motion in a tropical cyclone model. J. Atmos. Sci., 34, 1518-1527.

Jones, R.W., 1977b: A nested grid for a three-dimensional model of a tropical cyclone. J. Atmos. Sci., 34, 1528-1553.

Jordan, C.L., 1958: Mean soundings for the West Indies area. J. Meteor., 15, 91-97.

Jordan, C.L., and F.J. Schatzle, 1961: The "double-eye" of hurricane Donna. Mon. Wea. Rev., 89, 354-356.

Kasahara, A., 1977: Numerical integration of the global barotropic primitive equations with Hough harmonic expansions. J. Atmos. Sci., 34, 687-701.

Kasahara, A., 1978: Further studies on a spectral model of the global barotropic primitive equations with Hough harmonic expansions. J. Atmos. Sci., 35, 2043-2051. 
Kitade, T., 1980: Numerical experiments of tropical cyclones on a plane with variable Coriol is parameter. J. Meteor. Soc. Japan, 58, 471488.

Krylov, V.I., 1962: Approximate Calculation of Integrals. The Macmillan Company, New York, 357 pp.

Kuo, H.L., 1959: Motions of vorticies and circulating cylinder in shear flow with friction. J. Atmos. Sci., 26, 390-398.

Kurihara, Y., and R.E. Tuleya, 1981: A numerical simulation study on the genesis of a tropical storm. Mon. Wea. Rev., 109, 1629-1653.

Kwizak, M., and A.J. Robert, 1971: A semi-implicit scheme for grid point atmospheric models of the primitive equations. Mon. Wea. Rev., 99, 32-36.

Leith, C., 1980: Nonlinear normal mode initialization and quasigeostrophic theory. J.Atmos. Sci., 37, 958-968.

Lewis, B.M., and D.P. Jorgensen, 1978: Study of the dissipation of hurricane Gertrude (1974). Mon. Wea. Rev., 106, 1288-1306.

Lindzen, R.S., 1967: Planetary waves on beta-planes. Mon. Wea. Rev., 95, 441-451.

Machenhauer, B., 1977: On the dynamics of gravity oscillations in a shallow-water model, with application to normal model

initialization. Beitr. Phys. Atmos., 50, 253-271.

Machenhauer, B., 1979: Numerical methods used in atmospheric models, Vol. II, Chapter 3: The Spectral Method. GARP Publication Series No. 17, pp. 121-275.

Madala, R.V., and S.A. Piacsek, 1975: Numerical simulation of asymmetric hurricanes on a $\beta-p l a n e$ with vertical shear. Tellus, 27, 453-468.

Merril1, R.T., 1982: A comparison of large and small tropical cyclones. Dept. of Atmos. Sci. Paper No. 352, Colo. State Univ, Ft. Collins, $\mathrm{CO}, 75 \mathrm{pp}$.

Miller, B.I., 1958: The three-dimensional wind structure around a tropical cyclone. NHRP Report, No. 15, U.S. Dept. of Commerce, NOAA, NHRL 1320 S. Dixie Hwy., Coral Gables, FL 33146, 41 pp.

Neumann, C., and J. Pelissier, 1981: An analysis of Atlantic tropical cyclone forecast errors, 1970-1979. Mon. Wea. Rev., 109, 12481266.

Doyama, K.V., 1964: A dynamical model for the study of tropical cyclone development. Geofis. Int., 4, 187-198. 
Doyama, K.V., 1969a: Numerical simulation of the iffe cycle of tropical cyclones. J. Atmos. Sci., 26, 3-40.

Doyama, K.V., 1969b: Numerical simulation of tropical cyclones with an axisymmetric model. Proc. WM0/IUGG Symp. on Numerical Weather Prediction, Tokyo, 1968, II I :81-88.

Doyama, K.V., 1982: Conceptual evolution of the theory and modeling of the tropical cyclone. J. Meteor. Soc. Japan, 60, 369-379.

Orszag, S.A., 1970: Transform method for the calculation of vectorcoupled sums: Application to the spectral form of the vorticity equation. J. Atmos. Sci., 27, 890-895.

Orszag, S.A., 1979: Numerical Methods for PDE's: Spectral Methods for Problems in Complex Geometries, Academic Press, pp. 273-305.

Phillips, N.A., 1959: An example of nonlinear computational instability. The Atmosphere and the Sea in Motion, Rossby Memorial Volume, New York, Rockefeller Instit. Press, 501-504.

Puri, K., and W. Bourke, 1982: A scheme to retain the Hadley circulation during nonlinear normal mode initialization. Mon. Wea. Rev., 110, 327-335.

Reed, R.J., D.C. Norquist, and E.E. Recker, 1977: The structure and properties of African wave disturbances as observed during phase III of GATE. Mon. Wea. Rev., 105, 317-333.

Rosenthal, S.L., 1978: Numerical simulation of tropical cyclone development with latent heat release by the resolvable scales, I: model description and preliminary results. J.Atmos. Sci., 35, 258-271.

Rossby, C.G., 1948: On displacements and intensity changes of atmospheric vorticies. J. Marine Res., 7, 175-187.

Sadler, J.C., 1976: A role of the tropical upper tropospheric trough in early season typhoon development. Mon. Wea. Rev., 104, 1266-1278.

Sanders, F., and R.W. Burpee, 1968: Experiments in barotropic hurricane track forecasting. J. Appl. Meteor., 7, 313-323.

Sanders, F., A.C. Pike and J.P. Gaertner, 1975: A barotropic model for operational prediction of tracks of tropical storms. J. Appl. Meteor., 14, 265-280.

Schubert, W.H., J.J. Hack, P.L. Silva Dias and S.R. Fulton, 1980: Geostrophic adjustment in a axisymmetric vortex. J. Átmos. Sci., 37, 1464-1484.

Schubert, W.H., and J.J. Hack, 1982: Inertial stability and tropical cyclone development. J. Atmos. Sci., 39, 1687-1697. 
Shapiro, L.J., 1977: Tropical storm formation form easterly waves: A criterion for development. J. Atmos. Sci., 34, 1007-1021.

Shapiro, L.J., 1983: The asymmetric boundary layer flow under a

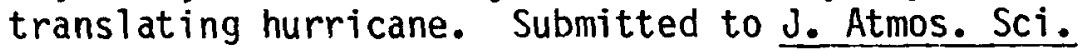

Shapiro, L.J., and H.E. Willoughby, 1982: The response of balanced hurricanes to local sources of heat and momentum. J. Atmos. Sci., 39, 378-394.

Shea, D.J., and W.M. Gray, 1973: The hurricanes inner core region, I: Symmetric and asymmetric structure. J.Atmos. Sci., 30, 1544-1564.

Sheets, R.C., 1969: Some mean hurricane soundings. J. Appl. Meteor., 8, $134-146$.

Silva Dias, P.L., W.H. Schubert, and M. DeMaria, 1983: Large-scale response of the tropical atmosphere to transient convection. Submitted to J. Atmos. Sci.

Simmons, A.J., and B.J. Hoskins, 1978: The life cycles of some nontinear baroclinic waves. J. Atmos. Sci., 35, 414-432.

Staniforth, A.N., and R. Daley, 1977: A finite-element formulation for the vertical discretization of sigma-coordinate primitive equation models. Mon. Wea. Rev., 105, 1108-1118.

Stevens, D.E., R.S. Lindzen, and L.J. Shapiro, 1977: A new model of tropical waves incorporating momentum mixing by cumulus convection. Dyn. Atmos. Oceans, $1,365-425$.

Temperton, C., and D.L. Williamson, 1979: Normal mode initialization for a multi-level gridpoint model. Tech. Rept. No. 11, European Centre for Medium Range Weather Forecasts, Shinfield Park, Reading, Engl and.

TuTeya, R.E., and Y. Kurihara, 1981: A numerical study on the effect of environmental flow on tropical storm genesis. Mon. Wea. Rev., 109, 2487-2506.

Veronis, G., and H. Stomel, 1956: The action of variable wind stresses on a stratified ocean. J. Mar. Res., 15, 43-75.

Williams on, D., 1976: Normal mode initialization procedure applied to forecasts with the global shallow water equations. Mon. Wea. Rev., 104, 195-206.

Willoughby, H.E., J.A. Clos, and M.G. Shoreibah, 1982: Concentric eye walls, secondary wind maxima, and the evolution of the hurricane vortex. J. Atmos. Sci., 39, 395-411.

Yanai, M., 1964: Formation of tropical cyclones. Rev. Geophys., $\underline{2}$, $367-414$. 


\section{APPENDIX A \\ THE USE OF FAST FOURIER TRANSFORM (FFT) ALGORITHMS \\ IN THE TRANSFORM METHOD}

When the transform method is used to calculate nonlinear terms in the spectral model, it is necessary to transform various combinations of the dependent variables from spectral space to physical space and back to spectral space at each time step. As described in chapter 3 , these transforms are performed by evaluating the following series

$$
\begin{aligned}
& f\left(x_{p}, y_{q}\right)=\sum_{\ell=-L} \sum_{k=-k}^{+L} \hat{f}_{k \ell} e^{i k x_{p}} e^{i \ell y_{q}}, \\
& \hat{f}_{k \ell}=\frac{1}{(3 N+1)} \frac{1}{(3 M+1)} \sum_{q=0}^{3 N} \sum_{p=0}^{3 M} f\left(x_{p}, y_{q}\right) e^{-i k x_{p}} e^{-i \ell y_{q}},
\end{aligned}
$$

where the transform grid points $x_{p}, y_{q}$ and wave numbers $k$ and $\ell$ are defined by

$$
\begin{array}{ll}
x_{p}=\left(\frac{L_{x}}{3 M+1}\right) p & p=0,1,2 \cdots 3 M \\
y_{q}=\left(\frac{L_{y}}{3 N+1}\right) q & q=0,1,2 \cdots 3 N
\end{array}
$$




$$
\begin{array}{lr}
k=\frac{2 \pi m}{L_{x}} & m=0, \pm 1, \pm 2 \cdots \pm M \\
\ell=\frac{2 \pi n}{L_{x}} & n=0, \pm 1, \pm 2 \cdots \pm N \\
K=\frac{2 \pi M}{L_{x}}, & L=\frac{2 \pi N}{L_{y}}
\end{array}
$$

and $L_{x}$ and $L_{y}$ are the east-west and north-south extents of the model domain. Equation (A.1) represents a truncated double Fourier series while (A.2) is the integral formula for the series amplitudes approximated by the trapezodial rule. The number of grid points was chosen so that (A.2) is exact if $f(x, y)$ is the product of two functions which are each represented by a truncated double Fourier series with maximum wave numbers $K$ and $L$ as defined by (A.7).

In order to use FFT algorithms to evaluate the series in (A.1) and (A.2), it is necessary to write these series in the form of a standard discrete Fourier transform which is given by

$$
F_{n}=\sum_{m=0}^{N D-1} F_{m} e^{\frac{ \pm 2 \pi i m n}{N D}} \quad \text { for } n=0,1,2 \cdots N D-1
$$

where ND is chosen to be an even number. In general, ND complex numbers $F_{m}$ are supplied and the algorithm returns ND complex numbers $F_{n}$ which are calculated using (A.8). If $F_{m}$ are real numbers, then $F_{n}$ have the following property: 


$$
F_{n}=F_{n-N D}^{*}
$$

where $F^{*}$ denotes complex conjugate. For this case it is only necessary to calculate $F_{n}$ for $n=0$ to $n=N D / 2$. Conversely, if the $F_{m}$ satisfy an equation analogous to (A.9) the corresponding $F_{n}$ values will be real. To make use of these properties, special routines have been written where if the $F_{m}$ values are all real, then the algorithm returns $F_{n}$ for $n=0$ to $n=N D / 2$ (real to complex) and if ND/2 complex values $F_{m}$ are supplied and it is assumed that $F_{m}=F_{m-N D}^{*}$, then the algorithm returns ND values of $F_{n}$ which are all real (complex to real).

Now, in order to write (A.2) so that the summations are in the form of $(A .8)$, first consider the inner summation which can be written as

$$
\hat{f}_{k}\left(y_{q}\right)=\frac{1}{(3 M+1)} \sum_{p=0}^{3 M} f\left(x_{p}, y_{q}\right) e^{-i k x_{p}} \text { for } k=-k \text { to } k=k
$$

Substituting for $x_{p}$ and $k$ from (A.3) and (A.5) gives

$$
\hat{f}_{m}\left(y_{q}\right)=\frac{1}{(3 M+1)} \sum_{p=0}^{3 M} f\left(x_{p}, y_{q}\right) e^{-\frac{2 \pi i m p}{3 M+1}} \text { for } m=-M \text { to } m=M
$$

(In this discussion, a subscript $m$ or $n$ is equivalent to a subscript $k$ or $\ell$ ). The summation in the above equation is of the form of (A.8) except that the summation runs from $p=0$ to $p=3 M$ and the summation must be evaluated for $m=-M$ to $m=M$. Since $f\left(x_{p}, y_{q}\right)$ represents a dependent variable in physical space, it is always real. For this case the Fourier series amplitudes calculated from (A.2) will satisfy the following relation: 


$$
\hat{f}_{m}\left(y_{q}\right)=\hat{f}_{-m}^{*}\left(y_{q}\right)
$$

Thus, it is only necessary to evaluate the summation in (A.10) for $m=0$ to $m=M$. This summation can then be evaluated using a real to complex FFT algorithm where the $3 M+1$ real values of $f\left(x_{p}, y_{q}\right)$ are supplied for each $y_{q}$ and $\frac{3 M+1}{2}$ complex values representing $f_{m}\left(y_{q}\right)$ are returned. Since only the values up to $m=M$ are needed, some extra computation is performed which would not be needed if the summation was evaluated directly. As will be discussed later, the efficiency of the FFT algorithm used more than compensates for this extra work. Since the summation in (A.10) must be evaluated for each $y_{q}$, an FFT routine available on NCAR's Cray-1 was used which performs multiple FFTs to make full use of the vector capabilities of the machine.

Once the values of $\hat{f}_{k}\left(y_{q}\right)$ have been calculated, the outer summation in (A.2) can be evaluated using

$$
\hat{f}_{k \ell}=\frac{1}{(3 N+1)} \sum_{q=0}^{3 N} \hat{f}_{k}\left(y_{q}\right) e^{-i \ell y q} \text { for } \ell=-L \text { to } \ell=L \text {. }
$$

Substituting for $y_{q}$ and $\ell$ from (A.4) and (A.6) gives

$$
\hat{f}_{m n}=\frac{1}{(3 N+1)} \sum_{q=0}^{3 N} \hat{f}_{m}\left(y_{q}\right) e^{\frac{-2 \pi i n q}{3 N+1}} \text { for } n=-N \text { to } n=+N \text {. }
$$

The above equation is again analogous to (A.8) except that the summation runs from $q=0$ to $q=3 N$ and the summation must be evaluated for $n=-N$ to $n=N$. For this case, however, $\hat{f}_{k}\left(y_{q}\right)$ is complex so that the summation must be evaluated for both the positive and negative $n$ values. The summation in (A.12) must then be evaluated using a complex to complex 
FFT algorithm where the input are the $3 N+1$ complex values of $\hat{f}_{m}\left(y_{q}\right)$ for each $\mathrm{m}$, and $3 \mathrm{~N}+1$ complex values are returned. The values which are returned by the algorithm correspond to a summation of the form of (A.8) with $N D=3 N+1$. Thus, the algorithm returns values of the summation in (A.12) for $n=0$ to $n=3 N$, while the values for $n=-N$ to $n=+N$ are needed. The values of $\hat{f}_{m n}$ for $n=0$ to $N$ are then computed directly by the FFT algorithm. To obtain the values of $f_{m n}$ for negative $n$, it is necessary to make use of the following relation

$$
\hat{f}_{m n}=\hat{f}_{m n+(3 N+1)} \text {. }
$$

The above relation comes from the property that $n$ in (A.12) can be replaced by $n+(3 N+1)$ since this is equivalent to multiplying the right side of this equation by $e^{-2 \pi i q}$ which equals unity since $q$ is an integer. For this part of the summation, the values of $f_{m n}$ for $|n|>N$ are not used so, again, some extra work is required in order to make use of the FFT algorithm. Similar to the real to complex case, special FFT algorithms which perform multiple FFTs were used to take advantage of the fact that (A.12) must be evaluated for each m.

Now consider the summations in (A.1). Using (A.4) and (A.6), the outer summation can be written as

$$
\hat{f}_{m}\left(y_{q}\right)=\sum_{n=-N}^{+N} \hat{f}_{m n} e^{\frac{2 \pi i n q}{3 N+1}} \quad \text { for } \quad q=0,1,2 \cdots 3 N
$$

The above summation can be written as

$$
\hat{f}_{m}\left(y_{q}\right)=\sum_{n=0}^{N} \hat{f}_{m n} e^{\frac{2 \pi i n q}{3 N+1}}+\sum_{n=-1}^{-N} \hat{f}_{m n} e^{\frac{2 \pi i n q}{3 N+1}} .
$$


Noting that $n$ in the second summation in (A.15) can be replaced by $n+(3 N+1)$, the above two summations can be combined to give

$$
\hat{f}_{m}\left(y_{q}\right)=\sum_{n=0}^{3 N} \hat{f}_{m n}^{\prime} e^{\frac{2 \pi i n q}{3 N+1}} \text { for } q=0,1,2 \cdots 3 N
$$

where

$$
\begin{array}{ll}
\hat{f}_{m n}^{\prime}=\hat{f}_{m n} & \text { for } \quad n=0,1 \quad N \\
\hat{f}_{m n}^{\prime}=0 \quad \text { for } \quad n=N+1, N+2, \cdots 2 N-1 \\
\hat{f}_{m n}^{\prime}=\hat{f}_{m n-(3 N+1)} \text { for } n=2 N, 2 N+1 \cdots 3 N
\end{array}
$$

Equation (A.16) is of the form of (A.8) with $N D=3 N+1$ so that the summation can be evaluated using a complex to complex FFT algorithm for each value of $\mathrm{m}$.

Once the values of $\hat{f}_{m}\left(y_{q}\right)$ have been calculated, the inner summation in (A.1) can be written as

$$
f\left(x_{p}, y_{q}\right)=\sum_{m=-M}^{+M} \hat{f}_{m}\left(y_{q}\right) e^{\frac{2 \pi i n p}{3 M+1}} \text { for } p=0,1,2 \cdots 3 M
$$

where (A.3) and (A.5) have been used. In the above summation $f\left(x_{p}, y_{q}\right)$ is real so that a complex to real FFT can be used. For this case it is only necessary to supply $\hat{f}_{m}\left(y_{q}\right)$ for $m=0,1, \ldots \frac{3 M+1}{2}$. Thus the summation in (A.17) is evaluated using a complex to real FFT where the values of $\hat{f}_{m}\left(y_{q}\right)$ for $m>M$ which are required are set to zero.

In summary, (A.16) and (A.17) are used to evaluate the transform from spectral to physical space and (A.10) and (A.12) are used to evaluate the transform form physical to spectral space where each 
summation is evaluated using an FFT algorithm. The FFT routines which were used to evaluate summations of the form of (A.8) required that the length of the transform ND be an even number with no factors greater than 5. Since the lengths of the transforms which appear in the transform method are either $3 M+1$ or $3 N+1$ where $M$ and $N$ are the Fourier series truncations, the restriction on ND is somewhat severe. In fact, the only values of $M$ and $N$ between 10 and 50 which could be used are 13, 21 and 33. In order to overcome this difficulty, the number of points on the transform grid was increased to $3 M+3$ by $3 N+3$ so that a larger selection of $M$ and $N$ values could be used. For this case the $M$ and $N$ values between 10 and 50 which can be used are $11,15,17,19,23,29$, $31,35,39,47$ and 49 . Although this extra grid resolution slightly decreases the transform speed, it also increases the accuracy of the nonlinear terms which are not quadratic, as discussed in chapter 3. As discussed previously, in order to use FFT algorithms, it is necessary to use longer transforms than would be needed if the transforms were evaluated directly. Basically this is because for the discrete Fourier transform the number of spectral amplitudes which are calculated is the same as the number of physical space values supplied. For the transform method, however, $3 N+1$ physical space grid points are required to evaluate the transform of quadratic nonlinear terms exactly, but the dependent variables are represented by $2 \mathrm{~N}+1$ Fourier modes (including positive and negative wavenumbers). In order to determine whether the FFT algorithms were efficient enough to compensate for this extra work, a Gaussian function was transformed from physical space to spectral space and back to physical space with and without the FFT algorithms. For the case without the FFT algorithms, the series were 
evaluated directly so that it was not necessary to increase the length of the transforms. For both cases, all the needed trigonometric functions were calcualted before the transform, so that these calculations were not included in the execution time. Fig. 3 shows the ratio of the execution time needed without the FFTs to the time needed with the FFTs as a function of the number of Fourier modes in each direction (the abscissa is $M+1$ or $N+1$ ). This figure shows that for $M=N=35$, the transforms were evaluated about 13 times faster using the FFTs even though it was necessary to increase the transform length. 
APPENDIX B

PROPERTIES OF THE NORMAL MODE TRANSFORM

In chapter 3 a normal mode transform was derived which transformed a column vector $\tilde{W}$ into a scalar. (For simplicity, the subscripts $j, k$ and $\ell$ which represent the vertical and horizontal modes will be dropped in this discussion.) This transform is given by

$$
W_{r}=\frac{1}{E_{r}}\left(\tilde{W}, \tilde{K}_{r}\right)
$$

where

$$
(\tilde{u}, \tilde{v})=\left(d^{2} u_{1} v_{1}^{*}+u_{2} v_{2}^{*}+u_{3} v_{3}^{*}\right)
$$

and $E_{r}$ is a normalization factor, $d^{2}$ is a proportionality constant, $\tilde{K}_{r}$ is a column vector which is the kernel of the transform and ()$^{*}$ denotes complex conjugate. The kernel $\tilde{K}_{r}$ was chosen to satisfy the following relation

$$
\tilde{A K}_{r}=-i \nu_{r} \tilde{K}_{r}
$$

where $\tilde{A}$ is a 3 by 3 matrix which represents the coefficients of the linear terms of the governing equations as written in the form of (3.55). Equation (B.3) implies that $\tilde{K}_{r}$ is an eigenvector of the matrix $\tilde{A}$ and that $-i \nu_{r}$ is the corresponding eigenvalue. If the matrix $\tilde{A}$ is skew-Hermitian with respect to the inner product (B.2) then the eigenvectors $\tilde{K}_{r}$ will be orthogonal. This is a desirable property since the inverse of the normal mode transform will be straightforward. In 
order for $\tilde{A}$ to be skew-Hermitian, it must satisfy the following relation

$$
(\tilde{A u}, \tilde{v})=-(\tilde{u}, \tilde{A v})
$$

Using (3.58) and (B.2), the inner products in the above equation can be written as

$$
\begin{aligned}
(\tilde{A u}, \tilde{v}) & =\left[g_{j} v_{3}^{*}\right] u_{1}+\left[\frac{-i \beta k}{\left(k^{2}+l^{2}\right)} v_{2}^{*}-f_{0} v_{3}^{*}\right] u_{2} \\
& +\left[-d^{2} H_{0}\left(k^{2}+l^{2}\right) v_{1}^{*}+f_{0} v_{2}^{*}-\frac{i \beta k}{\left(k^{2}+l^{2}\right)} v_{3}^{*}\right] u_{3} \\
-(\tilde{u}, \tilde{A v}) & =\left[d^{2} H_{0}\left(k^{2}+l^{2}\right) v_{3}^{*}\right] u_{1}+\left[\frac{-i \beta k}{\left(k^{2}+l^{2}\right)} v_{2}^{*}-f_{0} v_{3}^{*}\right] u_{2} \\
& +\left[-g_{j} v_{1}^{*}+f_{0} v_{2}^{*}-\frac{i \beta k}{\left(k^{2}+l^{2}\right)} v_{3}^{*}\right] u_{3} .
\end{aligned}
$$

Comparing (B.5) and (B.6) it can be seen that (B.4) will be satisfied provided that

$$
d^{2}=\frac{g_{j}}{H_{0}\left(k^{2}+\ell^{2}\right)}
$$

Now, (B.3) can be written as

$$
\left(\tilde{A}+i v_{r} \tilde{I}\right) \tilde{K}_{r}=0
$$

where $\tilde{I}$ is the 3 by 3 identity matrix. In order that the above equation have nontrivial solutions for $\tilde{K}_{r}$, the following condition must be met:

$$
\left|\tilde{A}+i \nu_{r} \tilde{I}\right|=0 \text {. }
$$

Evaluating the above determinant using (3.58) gives 


$$
\nu_{r}\left(\bar{v}_{r}^{2}-f_{0}^{2}\right)=g_{j} H_{0}\left(k^{2}+l^{2}\right) \bar{v}_{r}
$$

where

$$
\bar{v}_{r}=v_{r}-\frac{\beta k}{\left(k^{2}+\ell^{2}\right)}
$$

The three roots of (B.10) are proportional to the eigenvalues of the matrix $\tilde{A}$ and represent the frequencies of the normal modes of the linerized governing equations. Equation (B.8) represents three equations for the three components of $\tilde{K}_{r}$ which can be written as

$$
\begin{aligned}
-H_{0}\left(k^{2}+l^{2}\right) k_{r 3} & =-i \nu_{r} k_{r 1} \\
\frac{-i \beta k}{\left(k^{2}+l^{2}\right)} k_{r 2}+f_{0} k_{r 3} & =-i \nu_{r} k_{r 2} \\
g_{j} k_{r 1}-f_{0} k_{r 2}-\frac{i \beta k}{\left(k^{2}+l^{2}\right)} k_{r 3} & =-i \nu_{r} k_{r 3} .
\end{aligned}
$$

Since the magnitude of the kernel $\tilde{K}_{r}$ is arbitrary, one of the components of $\tilde{K}_{r}$ is also arbitrary. For convenience, $K_{r 1}$ was chosen to be equal to $f_{0}$. Then (B.11) can be used to give

$$
k_{r 3}=\frac{i \nu_{r} f_{0}}{H_{0}\left(k^{2}+l^{2}\right)}
$$

Substituting for $K_{r 1}$ and $K_{r 3}$ in (B.13) and solving for $K_{r 2}$ gives

$$
k_{r 2}=\frac{\beta k v_{r}-v_{r}^{2}\left(k^{2}+\ell^{2}\right)}{\left(k^{2}+l^{2}\right)^{2} H_{0}}+g_{j} .
$$

The kernel of the transform $\tilde{K}_{r}$ which satisfies (B.8) is then given by 


$$
\tilde{k}_{r}=\left(\begin{array}{c}
f_{0} \\
\frac{\beta k v_{r}-v_{r}^{2}\left(k^{2}+l^{2}\right)}{\left(k^{2}+l^{2}\right)^{2} H_{0}}+g_{j} \\
\frac{i v_{r} f_{0}}{H_{0}\left(k^{2}+l^{2}\right)}
\end{array}\right) .
$$

Since $\tilde{K}_{r}$ are the eigenvectors of the skew-Hermitian matrix $\tilde{A}$, they will be orthogonal provided that the corresponding eigenvalues are distinct. As was discussed in chapter 3 , this condition is satisfied since the three roots of (B.10) correspond to the frequencies of eastward and westward propagating gravity waves and to a westward propagating Rossby wave. The orthogonality condition can be written as

$$
\left(\tilde{K}_{r}, \tilde{K}_{r^{\prime}}\right)=E_{r} \delta_{r r^{\prime}}
$$

where $\delta_{r r}$ is the Kronecker delta. Now, provided that $E_{r}$ is defined as in (B.17), the normal mode transform and its inverse can be written as

$$
\begin{aligned}
& w_{r}=\frac{1}{E_{r}}\left(\tilde{W}, \tilde{K}_{r}\right) \\
& \tilde{W}=\sum_{r=1}^{3} w_{r} \tilde{K}_{r} .
\end{aligned}
$$

The above properties of the normal mode transform are valid for the external and internal vertical modes $(j=1,2)$. For the boundary layer modes $(j=0)$, an analogous transform of $\tilde{W}$ can be made. For this case, the transform is considerably simplier since the matrix $\tilde{A}$ is 2 by 2 as can be seen in (3.58). For this case, the normal mode transform and inverse are given by 


$$
\begin{aligned}
W_{r} & =\frac{1}{E_{r}}\left(\tilde{W}, \tilde{K}_{r}\right) \\
\tilde{W} & =\sum_{r=1} w_{r} \tilde{K}_{r}
\end{aligned}
$$

where

$$
\begin{aligned}
& (\tilde{u, v})=\left(u_{1} v_{1}^{*}+u_{2} v_{2}^{*}\right) \\
& \tilde{k}_{r}=\left(\begin{array}{c} 
\pm \\
1
\end{array}\right) \\
& \tilde{E}_{r}=\left(\tilde{k}_{r}, \tilde{k}_{r}\right)=2 .
\end{aligned}
$$


BIBLIOGRAPHIC DATA

SHEET

4. Tule and Juaricle
Repore No.

2.

3. Recipient's tecession No.

EXPERIMENTS WITH A SPECTRAL TROPICAL CYCLONE MODEL JuTy 1983

\begin{tabular}{l} 
7. Auchor(s) Mark DeMaria \\
\hline 9. Pcriormin: Organizacion Liame and Aduress \\
Department of Atmospheric Science \\
Colorado State University \\
Fort Collins, CO 80523
\end{tabular}

12 Sponsoring Organization Name ard duturess

National Science Foundation

Office of Naval Research

15. Supplemenary liotes

16. Abstracts

The three-layer balanced axisymmetric tropical cyclone model presented by 0oyama (1969) is generalized to three dimensions. The resultant primitive equations are solved using the spectral (Galerkin)method with Fourier basis functions on a doubly-

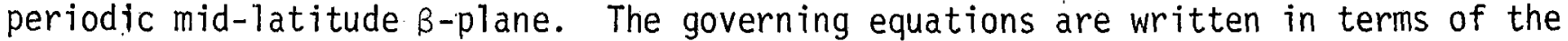
linear normal modes so that the linear terms can be evaluated exactly and nonlinear normal mode initialization can be applied. The transform method is used to calculate the nonlinear terms.

The model is run with several initial conditions and it is shown that many features of tropical cyclones found in nature, and many of 0oyama's results can be reproduced. The modal decomposition in terms of rotational and gravity modes is presented for a tropical cyclone simulation and it is shown that the gravity mode energy is much less than the rotational mode energy. It is also shown that nonlinear normal mode initialization prevents the excitation of gravity wave energy in a tropical cyclone simulation.

17. Key hords and Document Analysis. 17a. Descriptors

spectral models

tropical cyclone models

nonlinear normal mode initialization

mid-latitude beta-plane

17b. Idencipiers/Open-Ended Terms

17c. COStTI Field/Group

18. Avalisbilicy jedersent

\begin{tabular}{|c|c|}
\hline $\begin{array}{l}\text { 19. Security Class llas } \\
\text { Repert } \\
\text { Rorl a sifirn }\end{array}$ & $\begin{array}{l}\text { 21. . 10. o1 Pages } \\
224\end{array}$ \\
\hline 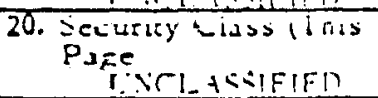 & 22. I'rice \\
\hline
\end{tabular}


